\title{
Akustische Strömung in Wasser durch Ultraschall im UHF-Bereich
}

\author{
DisSERTATION \\ zur Erlangung des mathematisch-naturwissenschaftlichen Doktorgrades \\ „Doctor rerum naturalium“ \\ der Georg-August-Universität Göttingen \\ im Promotionsprogramm ProPhys \\ der Georg-August University School of Science (GAUSS)
}

vorgelegt von

JuLian Konstantin EiseneR

aus

Tübingen

Göttingen 2020 
$\underline{\text { Betreuungsausschuss }}$

Prof. Dr. Jörg Enderlein,

Drittes Physikalisches Institut, Universität Göttingen

Dr. Robert Mettin,

Drittes Physikalisches Institut, Universität Göttingen

Prof. Dr. Ulrich Parlitz,

Biomedical Physics Group, MPIDS Göttingen

$\underline{\text { Mitglieder der Prüfungskommission }}$

Referent: $\quad$ Prof. Dr. Jörg Enderlein,

Drittes Physikalisches Institut, Universität Göttingen

Korreferent: Prof. Dr. Ulrich Parlitz,

Biomedical Physics Group, MPIDS Göttingen

Weitere Mitglieder der Prüfungskommission:

Dr. Robert Mettin,

Drittes Physikalisches Institut, Universität Göttingen

Prof. Dr. Wolfram Kollatschny,

Institut für Astrophysik, Universität Göttingen

Dr. Dieter Klopfenstein,

Drittes Physikalisches Institut, Universität Göttingen

Prof. Dr. Stefan Luther,

Biomedical Physics Group, MPIDS Göttingen

Tag der mündlichen Prüfung: 11. Dezember 2020 


\section{Inhaltsverzeichnis}

1. Einleitung 1

\begin{tabular}{ll}
2. & Theorie \\
\hline
\end{tabular}

2.1. Grundgleichungen der Fluiddynamik und Akustik . . . . . . . 3

2.1.1. Kontinuitätsgleichung. . . . . . . . . . . . 3

2.1.2. Impulsgleichung . . . . . . . . . . . . . . . . . . . . . . . . . 4

2.1.3. Energiegleichung . . . . . . . . . . . . . . . . . . . . . . 4

2.1.4. Zustandsgleichung . . . . . . . . . . . . . . . . . . . 4

2.2. Störungsrechnung . . . . . . . . . . . . . . . . 6

2.2.1. Bedingung für Approximation als kleine Störung. . . . 8

2.2.2. Trennung der Variablen . . . . . . . . . . 8

2.3. Lineare Akustik . . . . . . . . . . . . . . . . . . . . . . . . . . . . . . . . .

2.3.1. Lineare Wellengleichung . . . . . . . . . . . . . . . . . . 9 9

2.3.2. Schallabstrahlung und Nahfeld. . . . . . . . . . . . . 10

2.3.3. Öffnungswinkel des Schallfelds . . . . . . . . . . . . . . 10

2.3.4. Schallschnelle . . . . . . . . . . . . . . . . . . . 11

2.4. Eigenschaften von Schallwellen. . . . . . . . . . . . . . . . . . . . . . . . 11

2.4.1. Dämpfungs-Koeffizient . . . . . . . . . . . . . . . 12

2.4.2. Dämpfungslänge . . . . . . . . . . . . . . . . . . . . . . . . . . . . 12

2.5. Dimensionslose Kennzahlen . . . . . . . . . . . . . . . . . . . 14

2.5.1. Hydrodynamische Reynoldszahl . . . . . . . . . . . . . . 14

2.5.2. Akustische Reynoldszahl . . . . . . . . . . . . . . . . . 14

2.5.3. Machzahl . . . . . . . . . . . . . . . . . 16

2.6. Nichtlineare Akustik . . . . . . . . . . . . . . . . . . . . . . . 16

2.6.1. Westervelt-Gleichung . . . . . . . . . . . . . . 16

2.6.2. Burgers-Gleichung . . . . . . . . . . . . . . . . . . . . . 17

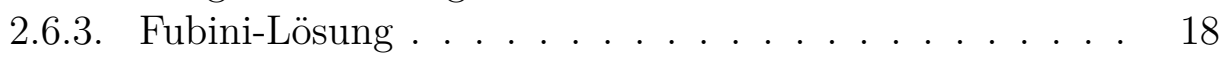

2.6.4. Schockdistanz . . . . . . . . . . . . . . . . . . . . 21

$2.6 .5 . \quad$ Fay-Lösung . . . . . . . . . . . . . . . . . . . . 23

2.6.6. Verbindung der Fay- und der Fubini-Lösung . . . . . . 25

2.6.7. Vergleich der Lösung der vollständigen Burgers-Gleichung mit Fay und Fubini . . . . . . . . . . . . . . . . . . . . . . . . . . .

2.7. Acoustic Streaming . . . . . . . . . . . . . . . . 31

2.7.1. Eckart-Streaming (Langsame Strömung zweiter Ordnung) 31

2.7.2. $\quad$ Akustische Strömung nach Zarembo. . . . . . . . . . . 32

2.7.3. Volumenkraft einer dissipierenden ebenen Welle . . . . 33 
2.7.4. Volumenkraft einer dissipierenden nichtlinearen ebenen Welle.................. . 36

2.7.5. Skalierung und Proportionalitäten . . . . . . . . . . . . 39

2.8. Kavitation . . . . . . . . . . . . . . . . . 40

2.8.1. Keller-Miksis-Modell . . . . . . . . . . . . . . . 40

2.8.2. Linearisierung des Keller-Miksis-Modells . . . . . . . . 41

2.8.3. Blake-Schwelle. . . . . . . . . . . . . . . . 43

2.8.4. Statische Blake-Schwelle . . . . . . . . . . . . . . 43

2.8.5. Dynamische Blake-Schwelle . . . . . . . . . . . . 45

3. Simulationen 51

3.1. Courant-Zahl . . . . . . . . . . . . . . . . 51

3.2. Lineare Schallausbreitung . . . . . . . . . . . . . . . 51

3.3. Nichtlineare Schallausbreitung . . . . . . . . . . . . . . . . 54

3.4. Einfache Näherung des Schallfelds in analytischer Form, ebene

dissipierende Welle . . . . . . . . . . . . . 58

3.4.1. Ebene dissipierende Welle in linearer Näherung . . . . 58

3.4.2. Ebene dissipierende Welle in nichtlinearer Näherung . . 60

3.5. Strömung durch Volumenkraft . . . . . . . . . . . . 63

3.5.1. Strömung durch Volumenkraft eines linear dissipierende Schallfeldes .................. 63

3.5.2. Strömung durch Volumenkraft eines nichtlinearen Schallfeldes.................. 66

$\begin{array}{ll}\text { 4. Experimente } & 73\end{array}$

4.1. Wandler . . . . . . . . . . . . . . . . . . . . . 73

4.1.1. Aufbau . . . . . . . . . . . . . . . 73

4.1.2. Messungen am Fraunhofer IBMT . . . . . . . . . . . 74

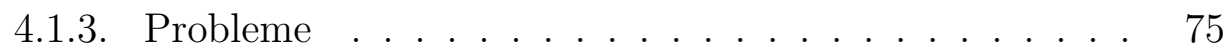

4.2. Strömungsmessung mit Tinte . . . . . . . . . . . . . . . . . . . . . . . 78

4.2.1. Versuchsaufbau ,Tinte ${ }^{6}$. . . . . . . . . . . . . . . 78

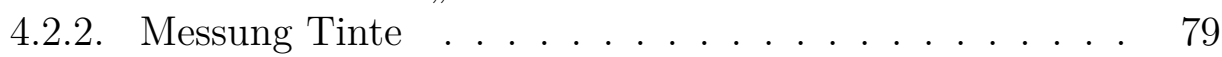

4.2.3. Ergebnisse Tinte . . . . . . . . . . . . . . . . . . 82

4.3. Particle Tracking Velocimetry (PTV) . . . . . . . . . . . . 83

4.3.1. Versuchsaufbau PTV . . . . . . . . . . . . . . 83

4.3.2. $\quad$ Ergebnisse PTV . . . . . . . . . . . . . . . . . . . . 84

4.4. Particle Image Velocimetry (PIV) . . . . . . . . . . . . . . . . . . 88

4.4.1. Versuchsaufbau PIV . . . . . . . . . . . . . . . . . . 89

$4.4 .2 . \quad$ Ergebnisse PIV . . . . . . . . . . . . . . . . . . . . . . 99

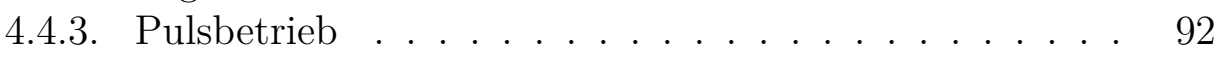

4.4.4. Betrieb mit kurzen Einzelpulsen . . . . . . . . . . . . . 94

4.4.5. Strömung an einer festen Grenzfläche . . . . . . . . . . 96

4.5. Chronoamperometrie . . . . . . . . . . . . . . . 98

4.5.1. Versuchsaufbau Chronoamperometrie . . . . . . . . 98 
4.5.2. $\quad$ Ergebnisse . . . . . . . . . . . . . . . . . . . . . 98 4.6. Experimente mit Luminol . . . . . . . . . . . . . . . . . . 101

4.6.1. Ergebnisse Luminol . . . . . . . . . . . . . . . . . 101

$\begin{array}{ll}\text { 5. Fazit } & 103\end{array}$

6. Ausblick 105

6.1. Tropfenerzeugung . . . . . . . . . . . . . . . . . . . 105

6.2. Mikropumpe / Flüssigkeitsjet . . . . . . . . . . . . . . . . 107

6.3. Strömung in Spalten . . . . . . . . . . . . . . . . . . 109

$\begin{array}{ll}\text { Literaturverzeichnis } & 111\end{array}$

\begin{tabular}{ll}
\hline A. Symbolliste & 117
\end{tabular}

\begin{tabular}{ll}
\hline B. Danksagung & 119
\end{tabular} 



\section{Einleitung}

Das Ziel dieser vorwiegend experimentellen Arbeit ist das Verständnis und die Untersuchung von Strömungen(,acoustic streaming“), die durch Schallfelder von neuartigen sehr hochfrequenten Schallwandlern in Flüssigkeiten erzeugt werden. Die Schallwandler liegen mit einer Resonanzfrequenz etwas unter ein Gigahertz im Frequenzbereich, der auf elektrischer Seite als UHF-Band (ultra high frequency) bezeichnet wird und $300 \mathrm{MHz}$ bis $3000 \mathrm{MHz}$ umfasst. Auf akustischer Seite spricht man ab $1 \mathrm{GHz}$ von „Hyperschall“ oder auch „Gigaschall“. Mit einem Durchmesser der Schallwandler von $200 \mu \mathrm{m}$ handelt es sich um ein kleinskaliges Phänomen, bei dem die räumliche Skala von der Wellenlänge des Schallfeldes mit $1,5 \mu \mathrm{m}$ bei $f=1 \mathrm{GHz}$ in Wasser, bis zur Reichweite der Strömung, die sich über einige Zentimeter erstrecken kann, reicht. Bei den Zeiten ist auch ein größerer Skalenbereich von Interesse. Auch hier stellt die Schallwelle mit einer Periode von einer Nanosekunde die untere Grenze dar. Das Ausbilden einer stationären Strömung kann bis zu Sekunden dauern, wobei Geschwindigkeiten von einigen m/s auftreten können.

Für die experimentelle Untersuchung der Strömung wird Tintenfront Verfolgung, Partikel Verfolgung (Particle Tracking), Particle Image Velocimetry (PIV) sowie Chronoamperometrie eingesetzt.

Simulationen des Schallfeldes, welches sich experimentell kaum messen lässt, helfen, das Phänomen zu verstehen und bilden die Grundlage für Strömungssimulationen, die mit den Experimenten verglichen werden.

Zunächst wird in Kapitel 2 in die nötigen theoretischen Grundlagen eingeführt. Numerische Simulationen werden in Kapitel 3 diskutiert, und in Kapitel 4 werden die Experimente vorgestellt. Nach einem Fazit in Kapitel 5 werden als Ausblick in Kapitel 6 Tests für verschiedene Anwendungen gezeigt. 
1. Einleitung 


\section{Theorie}

In diesem Abschnitt werden die für diese Arbeit wichtigen Grundlagen eingeführt. Hierbei wird zunächst die Fluiddynamik behandelt. Im Folgenden geht es über die Wellengleichung zur linearen und nichtlinearen Akustik, welche durch die hohen Schalldrücke im experimentellen Teil wichtig wird. Auch relevante entdimensionierte Größen werden behandelt, die sehr hilfreich sind, um Gleichungen zu vereinfachen und Darstellungen zu verallgemeinern sowie den Parameterbereich überblicken zu können.

Die hohen Schalldrücke machen neben der Nichtlinearität auch eine Abschätzung über das Auftreten von Kavitation nötig. Hier wird der für die Arbeit relevante Frequenzbereich untersucht und zu erwartende Blasengrößen als auch die Schalldruckschwelle für Kavitation abgeschätzt.

\subsection{Grundgleichungen der Fluiddynamik und Akustik}

Für die Herleitung einer Gleichung, die akustische Wellen in einem newtonschen, homogenen, viskosen und wärmeleitenden Fluid beschreibt, benötigt man die Kontinuitätsgleichung, Impulsgleichung, Energiegleichung und Zustandsgleichung, die im Folgenden gezeigt werden und die Grundlage für die Fluiddynamik darstellen.

\subsubsection{Kontinuitätsgleichung}

Wie von Blackstock in [1] beschrieben, ist die Kontinuitätsgleichung eine partielle Differentialgleichung, die für die Erhaltung der Masse sorgt. Die zeitliche Änderung der Dichte $\rho$ eines Fluidelementes muss gleich der ein- sowie ausgeflossenen Masse sein. Als vektorielle Gleichung formuliert, ergibt sich die Gleichung (2.1.1):

$$
\frac{\partial \rho}{\partial t}+\nabla \cdot(\rho \vec{u})=0
$$

Wie üblich steht $t$ für die Zeit und $\vec{u}$ für die Geschwindigkeit. 


\subsubsection{Impulsgleichung}

Die Navier-Stokes-Gleichung, die unabhängig von Navier und Stokes hergeleitet wurde, stellt die Impulsgleichung für ein Kontinuum dar. Wenn man die Viskosität eines Fluides berücksichtigt, ist die Gleichung (2.1.2) eine gängige Schreibweise, wie sie z.B. in [2] verwendet wird:

$$
\rho \frac{\partial \vec{u}}{\partial t}+\rho(\vec{u} \cdot \nabla) \vec{u}=-\nabla p+\mu \Delta \vec{u}+\left(\eta+\frac{\mu}{3}\right) \nabla(\nabla \cdot \vec{u})+\vec{F}
$$

Dabei steht $p$ für den Druck, $\mu$ für die dynamische Viskosität und $\eta$ für die Volumenviskosität. Externe Kräfte werden durch $\vec{F}$ berücksichtigt und haben die Form einer Volumenkraft mit der Einheit $\mathrm{N} / \mathrm{m}^{3}$ wie z.B. der Gravitationskraft $\vec{F}_{g}=\rho \vec{g}$. Die akustische Strömung wird später auch durch eine Volumenkraft, die aus dem Schallfeld resultiert, mit der Fluiddynamik gekoppelt.

Eine detaillierte Herleitung der Gleichung (2.1.2) kann man z.B. in [3] finden.

\subsubsection{Energiegleichung}

Die Energie im Fluid setzt sich aus der kinetischen Energie, der Bruttobewegung des Fluids und der thermodynamischen inneren Energie zusammen [4] [5] . Die folgende Gleichung deckt die Dissipation von mechanischer Energie und ihre Transformation in Wärme ab:

$$
\rho T\left[\frac{\partial s}{\partial t}+(\vec{u} \cdot \nabla) s\right]=\mu\left(\frac{\partial u_{i}}{\partial x_{k}}+\frac{\partial u_{k}}{\partial x_{i}}-\frac{2}{3} \frac{\partial u_{j}}{\partial x_{j}} \delta_{i k}\right)^{2}+\eta(\nabla \cdot \vec{u})^{2}+\kappa \nabla^{2} T
$$

Dabei steht $T$ für die Temperatur, $s$ für die Entropie, $\kappa$ die thermische Leitfähigkeit und $\delta_{i k}$ ist das Kronecker Delta.

Der Term $\mu\left(\frac{\partial u_{i}}{\partial x_{k}}+\frac{\partial u_{k}}{\partial x_{i}}-\frac{2}{3} \frac{\partial u_{j}}{\partial x_{j}} \delta_{i k}\right)^{2}+\eta(\nabla \cdot \vec{u})^{2}$ ist die Rate der Wärmeproduktion durch Reibungsdissipation von makroskopischen Bewegungen.

Der Fluss von Wärmeenergie pro Volumeneinheit durch molekulare Diffusion ist durch $\kappa \nabla^{2} T$ gegeben.

\subsubsection{Zustandsgleichung}

Die Zustandsgleichung beschreibt die Kompression und Expansion eines Fluides. Um diesen Vorgang zu beschreiben, wie es in [6] gemacht wird, benötigt man eine Relation zwischen dem Druck $p$, der Dichte $\rho$ und der spezifischen Entropie $s$. Bei akustischen Phänomenen kann die Variation der Entropie meist vernachlässigt werden und die Rechnungen können mit konstanter Entropie durchgeführt werden. 
Für die lokale isentropische Schallgeschwindigkeit $c$ in einem Fluid gilt

$$
c^{2}=\left.\left(\frac{\partial p}{\partial \rho}\right)\right|_{s}
$$

wobei der Index $s$ bedeutet, dass bei der Rechnung die Entropie konstant gehalten wird.

Ein ideales Gas gehorcht der Gleichung

$$
p=p_{0}\left(\frac{\rho}{\rho_{0}}\right)^{\gamma}
$$

mit der Referenzdichte $\rho_{0}$, dem Umgebungsdruck $p_{0}$ und dem Adiabatenexponent (Isentropenexponent) $\gamma=\frac{c_{p}}{c_{v}}$, der sich aus der Wärmekapazität bei konstantem Druck $c_{p}$ und der Wärmekapazität bei konstantem Volumen $c_{v}$ zusammensetzt. Für die Beschreibung von Flüssigkeiten wird oft eine ähnliche Gleichung mit empirischen Parametern verwendet. Für Wasser setzt man z.B. die Tait-Gleichung ein.

Die Tait Gleichung (2.1.5) ist ähnlich wie die Gleichung für ein ideales Gas aufgebaut:

$$
\begin{aligned}
p & =P\left(\frac{\rho}{\rho_{0}}\right)^{\gamma_{L}}-Q \\
\frac{p+Q}{p_{\infty}+Q} & =\left(\frac{\rho}{\rho_{0}}\right)^{\gamma_{L}} \\
P & =p_{\infty}+Q
\end{aligned}
$$

Die Parameter $Q$ und $\gamma_{L}$ müssen experimentell bestimmt werden. Für Wasser finden sich in der Literatur die Werte $\gamma_{L}=7$ und $Q=3000$ bar.

In der Zustandsgleichung lässt sich der Druck auch durch eine Taylor-Reihe als Funktion der Dichte und Entropie entwickeln:

$$
p-p_{0}=\left(\frac{\partial p}{\partial \rho}\right)_{s, \rho=\rho_{0}} \cdot\left(\rho-\rho_{0}\right)+\frac{1}{2}\left(\frac{\partial^{2} p}{\partial \rho^{2}}\right)_{s, \rho=\rho_{0}} \cdot\left(\rho-\rho_{0}\right)^{2}+\ldots
$$

oder

$$
p-p_{0}=A \frac{\rho-\rho_{0}}{\rho_{0}}+\frac{B}{2}\left(\frac{\rho-\rho_{0}}{\rho_{0}}\right)^{2}+\frac{C}{6}\left(\frac{\rho-\rho_{0}}{\rho_{0}}\right)^{3}+\ldots
$$


mit

$$
\begin{aligned}
& A=\left.\rho_{0}\left(\frac{\partial p}{\partial \rho}\right)\right|_{s, \rho=\rho_{0}}=\rho_{0} c_{0}^{2} \\
& B=\left.\rho_{0}^{2}\left(\frac{\partial^{2} p}{\partial \rho^{2}}\right)\right|_{s, \rho=\rho_{0}} \\
& C=\left.\rho_{0}^{3}\left(\frac{\partial^{3} p}{\partial \rho^{3}}\right)\right|_{s, \rho=\rho_{0}}
\end{aligned}
$$

Da die Terme höherer Ordnung in der Regel vernachlässigt werden können, wird bereits $C$ nicht weiter verwendet.

Der Parameter der Nichtlinearität $B / A$ wird für ein ideales Gas durch

$$
\frac{B}{A}=\gamma-1
$$

beschrieben.

Falls $B=0$ ist oder gesetzt wird, spricht man von linearer Akustik. Für $B \neq 0$ bekommt man nichtlineare Effekte, deren Größe durch das Verhältnis von $B$ zu $A$ bestimmt wird. Für andere Fluide als ein ideales Gas gibt es für $B / A$ experimentell ermittelte Literaturwerte.

Öfters wird auch der Koeffizient für Nichtlinearität

$$
\beta=1+\frac{B}{2 A}
$$

verwendet. Durch das Verhältnis von $B / A$ wird die Schallgeschwindigkeit $c$ durch eine lineare Funktion in Abhängigkeit von der Amplitude der Partikelgeschwindigkeit $u$ korrigiert:

$$
c=c_{0}+\frac{B}{A} u
$$

Man erkennt, dass die Schallgeschwindigkeit bei positivem $\frac{B}{A}$ für größere Schnellen (und damit auf für größere Wechseldrücke) zunimmt. Für Wasser unter atmosphärischem Druck und bei $20^{\circ} \mathrm{C}$ findet sich in [7] ein Wert von $\frac{B}{A}=5$, was einem $\beta=3,5$ entspricht.

\subsection{Störungsrechnung}

Unter Annahme einer infinitesimalen kleinen Amplitude kann die Störungsrechnung genutzt werden, um die Gleichungen zu linearisieren [4, 8, 9, 10].

Die Geschwindigkeit, der Druck und die Dichte werden wie folgend in Reihen 
entwickelt:

$$
\begin{aligned}
\vec{u} & =\overrightarrow{u_{0}}+\epsilon \overrightarrow{u_{1}}+\epsilon^{2} \overrightarrow{u_{2}}+\epsilon^{3} \ldots \\
p & =p_{0}+\epsilon p_{1}+\epsilon^{2} p_{2}+\epsilon^{3} \ldots \\
\rho & =\rho_{0}+\epsilon \rho_{1}+\epsilon^{2} \rho_{2}+\epsilon^{3} \ldots
\end{aligned}
$$

Der Index 0 gibt den ungestörten Zustand im Gleichgewicht an, also den Umgebungsdruck $p_{0}$, bzw die Fluiddichte $\rho_{0}$ und die Fluidgeschwindigkeit in Ruhe, also $\vec{u}_{0}=0.1$ Mit dem Index 1 wird die Störung erster Ordnung bezeichnet bzw. mit 2 die Störung zweiter Ordnung. Durch Einsetzen von (2.2.1) in die Kontinuitätsgleichung 2.1.1) sowie in die Navier-Stokes-Gleichung (2.1.2) folgt für die erste Näherung die nur Therme $\epsilon<2$ berücksichtigt:

$$
\begin{array}{r}
\frac{\partial \rho_{1}}{\partial t}+\rho_{0} \nabla \cdot \overrightarrow{u_{1}}=0 \\
\rho_{0} \frac{\partial \vec{u}_{1}}{\partial t}=-\nabla p_{1}+\mu \nabla^{2} \vec{u}_{1}+\left(\eta+\frac{\mu}{3}\right) \nabla \nabla \cdot \vec{u}_{1}
\end{array}
$$

Aus der Zustandsgleichung folgt für einen adiabatischen Prozess, dass $p_{1}=$ $c_{0}^{2} \rho_{1}$ und damit unabhängig von der Fluidtemperatur erster Ordnung ist, womit die Entwicklung der Energiegleichung 2.1.3 in erster Ordnung

$$
\left[\rho_{0} T_{0} \frac{\partial s_{1}}{\partial t}\right]_{s_{1}=s_{0}}=0
$$

ergibt.

Für die Näherung zweiter Ordnung der Gleichungen ergibt sich:

$$
\begin{array}{r}
\frac{\partial \rho_{2}}{\partial t}+\nabla \cdot\left(\rho_{0} \vec{u}_{2}\right)+\nabla \cdot\left(\rho_{1} \vec{u}_{1}\right)=0 \\
\rho_{0} \frac{\partial \vec{u}_{2}}{\partial t}+\rho_{1} \frac{\partial \vec{u}_{1}}{\partial t}+\rho_{0}\left(\vec{u}_{1} \cdot \nabla\right) \vec{u}_{1}=-\nabla p_{2}+\mu \nabla^{2} \vec{u}_{2}+\left(\eta+\frac{\mu}{3}\right) \nabla \nabla \cdot \vec{u}_{2}
\end{array}
$$

Mit den Näherungen lassen sich Wellengleichungen erster bzw. zweiter Ordnung herleiten, was im Folgenden skizziert wird.

\footnotetext{
${ }^{1}$ An anderer Stelle wird $u_{0}$ als Anregungspartikelgeschwindigkeit (Schallschnelle) des Schallfeldes verwendet und sollte nicht mit der Fluidströmung verwechselt werden.
} 


\subsubsection{Bedingung für Approximation als kleine Störung}

Um das Schallfeld als kleine Auslenkung aus der Ruhelage behandeln zu können, muss es folgende drei Bedingungen erfüllen[1]:

$$
\begin{aligned}
& |\rho| \ll \rho_{0} \\
& |p| \ll \rho_{0} c_{0}^{2} \\
& |u| \ll c_{0}
\end{aligned}
$$

Man beachte, dass für den Druck $p$ nicht die Annahme $|p| \ll p_{0}$ gilt, sondern die oben genannte $|p| \ll \rho_{0} c_{0}^{2}$, insbesondere können auch negative Druckwerte auftreten.

Für Wasser muss diese „kleine“ Störung des Drucks deutlich kleiner sein als $\approx$ $2200 \mathrm{MPa}$ bzw. 22000 bar. Jedoch muss abseits dieser Bedingung noch darauf geachtet werden, ob Kavitation eine Rolle spielen kann, da das Aufreißen der Flüssigkeit je nach Frequenz und Substanz schon deutlich früher auftreten kann. Diese Betrachtung wird in Abschnitt 2.8 gemacht.

\subsubsection{Trennung der Variablen}

Nach [6] ist die Berechnung von Schall getrennt vom Wärmetransport möglich, wenn

$$
\begin{aligned}
& \omega \ll \frac{\rho c_{0}^{2}}{(4 / 3) \mu+\eta} \\
& \omega \ll \frac{\rho c_{0}^{2} c_{p}}{\kappa}
\end{aligned}
$$

gilt. Mit den Werten aus Tabelle 2.1

Tab. 2.1.: Parameter von Wasser für die Gleichungen (2.2.7) und (2.2.8).

\begin{aligned} \hline \hline$\omega & =2 \cdot \pi f & &$ Kreisfrequenz \\ $\rho & \approx 1000 \mathrm{~kg} / \mathrm{m}^{3} & &$ Dichte von Wasser \\ $c_{0} & \approx 1500 \mathrm{~m} / \mathrm{s} & &$ Schallgeschwindigkeit vont Wasser \\ $\mu & \approx 0,001 \mathrm{~Pa} \cdot \mathrm{s} & &$ Scherviskosität von Wasser \\ $\eta & \approx 2,1 \cdot \mu & &$ Volumenviskosität von Wasser \\ $c_{p} & \approx 4183 \frac{\mathrm{J}}{\mathrm{kg} \cdot \mathrm{K}} & &$ Spezifische Wärme bei konstantem Druck von Wasser \\ $\kappa & \approx 0,6 \frac{\mathrm{W}}{\mathrm{m} \cdot \mathrm{K}} & &$ Wärmeleitfähigkeit von Wasser \end{aligned}


ergibt sich für die rechten Seiten von Gleichung 2.2.7 und 2.2.8

$$
\begin{aligned}
\frac{\rho c^{2}}{(4 / 3) \mu+\eta} & \approx 6,5 \cdot 10^{11} \mathrm{~Hz} \\
\frac{\rho c_{0}^{2} c_{p}}{\kappa} & \approx 1,5 \cdot 10^{13} \mathrm{~Hz} .
\end{aligned}
$$

Da die höchste in dieser Arbeit verwendete Schallfrequenz unter $f=1 \mathrm{GHz}=$ $1 \cdot 10^{9} \mathrm{~Hz}$ liegt, ist die Annahme aus Gleichung (2.2.7) sowie 2.2.8 gerechtfertigt. Auch wenn man berücksichtigt, dass es durch Nichtlinearitäten zu einigen Harmonischen der Anregungsfrequenz kommt, behält die Annahme ihre Gültigkeit.

\subsection{Lineare Akustik}

Durch Vernachlässigen von Termen höherer Ordnung und durch Linearisieren der Gleichungen können weite Bereiche der Akustik mit der linearen Wellengleichung beschrieben werden. Hier werden die Eigenschaften eines Kolbenstrahlers betrachtet, um die Länge des Nahfelds sowie die Richtcharakteristik des Schallwandlers zu berechnen.

\subsubsection{Lineare Wellengleichung}

Der Herleitung von Howe [5] folgend, kann mit der Näherung erster Ordnung der Kontinuitätsgleichung (2.2.2) in der Impulsgleichung in erster Näherung (2.2.3) (unter Vernachlässigung der Viskosität) die Geschwindigkeit $\vec{u}$ eliminiert werden und man erhält

$$
\frac{\partial^{2} \rho_{1}}{\partial t^{2}}-\nabla^{2} p_{1}=0
$$

Mit Hilfe der Zustandsgleichung (2.1.6) kann man für einen adiabatischen Prozess die Dichte durch eine Funktion des Drucks ersetzen und erhält somit die akustische Wellengleichung für den Wechseldruck 1. Ordnung:

$$
\left(\frac{1}{\rho_{0} c_{0}^{2}} \frac{\partial^{2} p}{\partial t^{2}}-\frac{1}{\rho_{0}} \nabla^{2} p\right)=0
$$

Wird noch die Viskosität berücksichtigt, lässt sich das Schallfeld durch

$$
\frac{1}{\rho_{0} c_{0}^{2}} \frac{\partial^{2} p}{\partial t^{2}}+\nabla \cdot\left(-\frac{1}{\rho_{0}}(\nabla p)+\frac{1}{\rho_{0} c_{0}^{2}}\left(\frac{4 \mu}{3}+\eta\right) \frac{\partial \nabla p}{\partial t}\right)=0
$$

beschreiben. Mit einem weiteren Term kann ebenfalls noch die thermische 
Leitfähigkeit berücksichtigt werden. Dann ergibt sich

$$
\frac{1}{\rho_{0} c_{0}^{2}} \frac{\partial^{2} p}{\partial t^{2}}+\nabla \cdot\left(-\frac{1}{\rho_{0}}(\nabla p)+\frac{1}{\rho_{0} c_{0}^{2}}\left(\frac{4 \mu}{3}+\eta+\frac{(\gamma-1) \kappa}{c_{p}}\right) \frac{\partial \nabla p}{\partial t}\right)=0
$$

\subsubsection{Schallabstrahlung und Nahfeld}

Das Nahfeld einer Schallquelle erstreckt sich bis zur Fresnellänge $L_{f}$. Die Fresnellänge lässt sich mit Gleichung (2.3.5) berechnen [11]:

$$
L_{f}=\frac{D_{s}^{2}}{4 \lambda}
$$

Hierbei ist $D_{s}$ der Durchmesser einer kreisförmigen abstrahlenden Fläche mit homogener Geschwindigkeit (Kolbenstrahler). Bei den hier betrachteten Schallwandlern ergeben sich die Werte aus Tabelle 2.2 .

\begin{tabular}{|c|c|c|}
\hline$\overline{D_{s}}=$ & $200 \mu \mathrm{m}$ & Durchmesser Schallquelle \\
\hline$c=$ & $1500 \mathrm{~m} / \mathrm{s}$ & Schallgeschwindigkeit \\
\hline$f=$ & $750 \mathrm{MHz}$ & Schallfrequenz \\
\hline$\lambda=$ & $2 \mu \mathrm{m}$ & Wellenlänge \\
\hline$L_{f}=$ & $5 \mathrm{~mm}$ & Länge Nahfeld \\
\hline
\end{tabular}

Tab. 2.2.: Nahfeldparameter

Es ergibt sich eine Fresnellänge von $5 \mathrm{~mm}$. Damit ist die Fresnellänge in dem Parameterbereich dieser Arbeit deutlich länger als die Schockdistanz sowie die Dissipationslänge, die beide weiter unten beschrieben werden.

\subsection{3. Öffnungswinkel des Schallfelds}

Der Öffnungswinkel $\theta_{o}$ des Abstrahlkegels einer runden Schallquelle lässt sich mit

$$
\sin \theta_{o}=1,22 \frac{\lambda}{D_{s}}
$$

berechnen [12, 13$]$.

Mit der Gleichung (2.3.6) ergibt sich für einen Schallwandler mit einem Durchmesser von $D_{s}=200 \mu \mathrm{m}$ bei einer Frequenz von $f=750 \mathrm{MHz}$ in Wasser, also mit einer Schallgeschwindigkeit $c \approx 1500 \mathrm{~m} / \mathrm{s}$, einer Wellenlänge von $\lambda \approx 2 \mu \mathrm{m}$, ein Öffnungswinkel von $\theta_{o}=0,7^{\circ}$.

Der Wandlerdurchmesser $D_{s}$ ist deutlich größer als die Wellenlänge $\lambda$, was zu einem stark gerichteten Schallfeld führt. Mit der Gleichung (2.3.7) lässt sich die Richtcharakteristik $R$ berechnen [12]. Sie wird in Abbildung 2.1 für die 
Werte $k=2 \pi \cdot f / c$ mit $f=15 \mathrm{MHz}$ und $f=750 \mathrm{MHz}$ gezeigt.

$$
R(\theta)=\frac{2 J_{1}\left(k D_{s} \sin \theta\right)}{k D_{s} \sin \theta}
$$

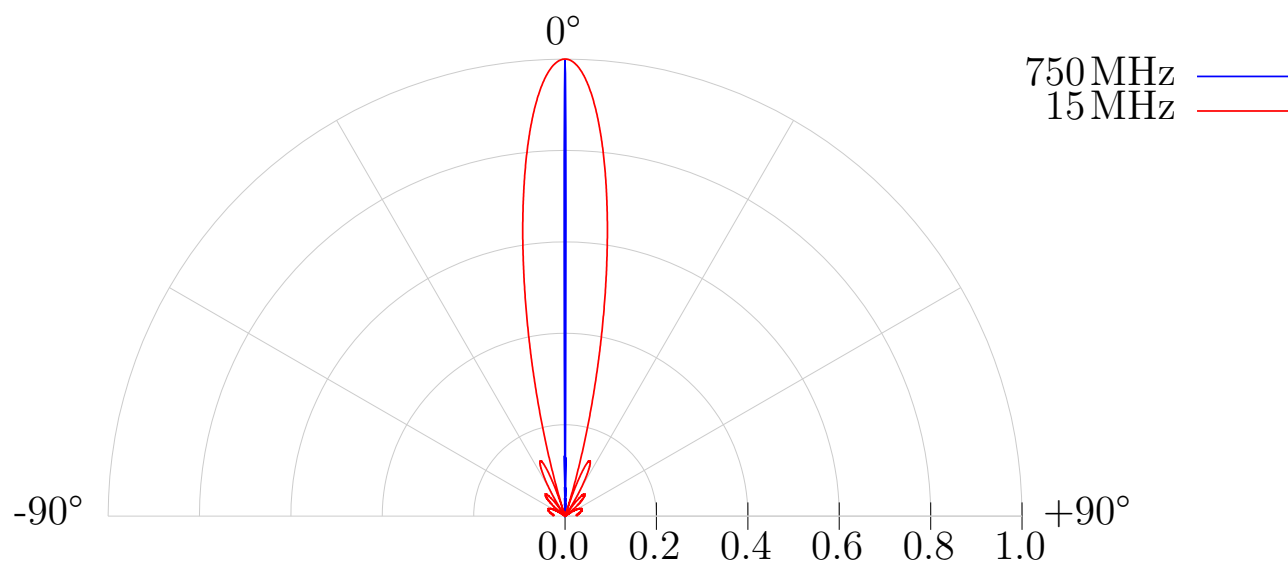

Abb. 2.1.: Polardiagramm der Richtcharakteristik eines kreisförmigen Schallwandlers mit einem Durchmesser $D_{s}=200 \mu \mathrm{m}$ für eine Frequenz von $f=750 \mathrm{MHz}$ (blau) und $f=15 \mathrm{MHz}$ (rot).

Wie schon durch den Öffnungswinkel zu erwarten war, zeigt auch die Abbildung 2.1 eine starke Richtungsabhängigkeit der Schallintensität, die mit steigender Frequenz noch zunimmt. Bei $750 \mathrm{MHz}$ wird nahezu ideal senkrecht zur Schallwandlermembran abgestrahlt.

\subsubsection{Schallschnelle}

In der linearen Akustik gibt es für eine ebene Welle eine Relation zwischen dem Schalldruck und der Schallschnelle, also der Amplitude der oszillatorischen Teilchenbewegung $u$ in der Welle, wie in Gleichung (2.3.8) formuliert [6]:

$$
p=\rho \cdot c_{0} \cdot u
$$

Mit der Dichte $\rho$ und der Schallgeschwindigkeit $c_{0}$ lässt sich der Schalldruck in eine Partikelgeschwindigkeit umrechnen und umgekehrt.

\subsection{Eigenschaften von Schallwellen}

Hier werden zwei weitere Phänomene der Schallwellen erörtert, die hier relevant sind. Zum einen kommt es bei der Ausbreitung der Schallwelle zur 
Dämpfung, also einer Abnahme der Intensität, die durch einen DämpfungsKoeffizienten beschrieben werden kann. Bei hohen Intensitäten kann es auch zu nichtlinearen Effekten kommen, die zu einer Verformung der anfangs sinusförmigen Schallwelle hin zu einer Stoßwelle mit einer vertikalen Tangente führen.

\subsubsection{Dämpfungs-Koeffizient}

Wie von Moudjed [13] vorgeschlagen wird beim akustischen Dämpfungs-Koeffizienten $\alpha$ meistens angenommen, dass er drei Komponenten hat.

Die eine Komponente resultiert aus der dynamischen Viskosität $\mu$, die auch als Scherviskosität bezeichnet wird. Ebenso hat die Volumenviskosität $\eta$ einen Anteil am Dämpfungskoeffizienten. Einen weiteren Beitrag liefern thermische Effekte.

Von Nasch [14] wird folgender Ausdruck für den Dämpfungskoeffizienten vorgeschlagen:

$$
N_{\alpha}=\frac{\alpha}{f^{2}}=\frac{2 \pi^{2}}{\rho c^{3}}\left(\frac{4}{3} \mu+\eta+\frac{c^{2} \beta_{t}^{2} \kappa T}{c_{p}^{2}}\right)
$$

mit den Werten aus Tabelle 2.3 für Wasser bei $20^{\circ} \mathrm{C}$ :

Tab. 2.3.: Mit Werten für die Gleichung (2.4.1)

\begin{tabular}{|c|c|c|}
\hline$\rho=$ & $998,2 \mathrm{~kg} / \mathrm{m}^{3}$ & Dichte \\
\hline$\mu=$ & $0,001 \mathrm{Pas}$ & Scherviskosität \\
\hline$\eta=$ & $2,1 \cdot \mu$ bis $3 \cdot \mu$ & Volumen-Viskosität \\
\hline$\beta_{t}=$ & $2,07 \cdot 10^{-4} \mathrm{~K}^{-1}$ & thermischer Expansions-Koeffizient \\
\hline$c_{p}=$ & $4180 \mathrm{~J} /(\mathrm{K} \mathrm{kg})$ & spezifische Wärme \\
\hline$c_{0}=$ & $1480 \mathrm{~m} / \mathrm{s}$ & Schallgeschwindigkeit \\
\hline$\kappa=$ & $0,61 \mathrm{~W} /(\mathrm{mK})$ & Wärmeleitfähigkeit \\
\hline$N_{\alpha} \approx$ & $226 \cdot 10^{-16} \mathrm{~s}^{2} / \mathrm{m}$ & $\alpha / f^{2}$ \\
\hline
\end{tabular}

Den kleinsten Anteil haben thermischen Effekte. Mit $\frac{c^{2} \beta_{t}^{2} \kappa T}{c_{p}^{2}}=0,96 \cdot 10^{-3} \mathrm{mPas}$ ist dies der kleinste Term.

Gegenüber dem Beitrag von $\frac{4}{3} \mu=1,33 \mathrm{mPas}$ aus der dynamischen Viskosität und $\eta=2,1 \mathrm{mPas}$ bis $3 \mathrm{mPas}$ resultierend aus der Volumen-Viskosität kann der thermische Anteil eher vernachlässigt werden.

\subsubsection{Dämpfungslänge}

Die Dämpfungs- oder auch Dissipationslänge bezeichnet die Strecke $x_{d}$, auf der die Intensität $I$ des Schallfeldes um den Faktor $e \approx 2,718 \ldots$ gedämpft wird. Der Faktor $e$ wurde aus rechnerischer Bequemlichkeit gewählt, da mit 
dem Dämpfungskoeffizienten $\alpha$ aus Abschnitt 2.4.1 Folgendes gilt:

$$
\begin{aligned}
I_{x} & =I_{0} \cdot e^{-\alpha x} \\
I_{0} / e & =I_{0} \cdot e^{-\alpha x_{d}} \\
\ln \left(\frac{1}{e}\right) & =-\alpha x_{d} \\
x_{d} & =\frac{1}{\alpha}
\end{aligned}
$$

In Abbildung 2.2 wird der Zusammenhang zwischen Frequenz und Dissipationslänge gezeigt.

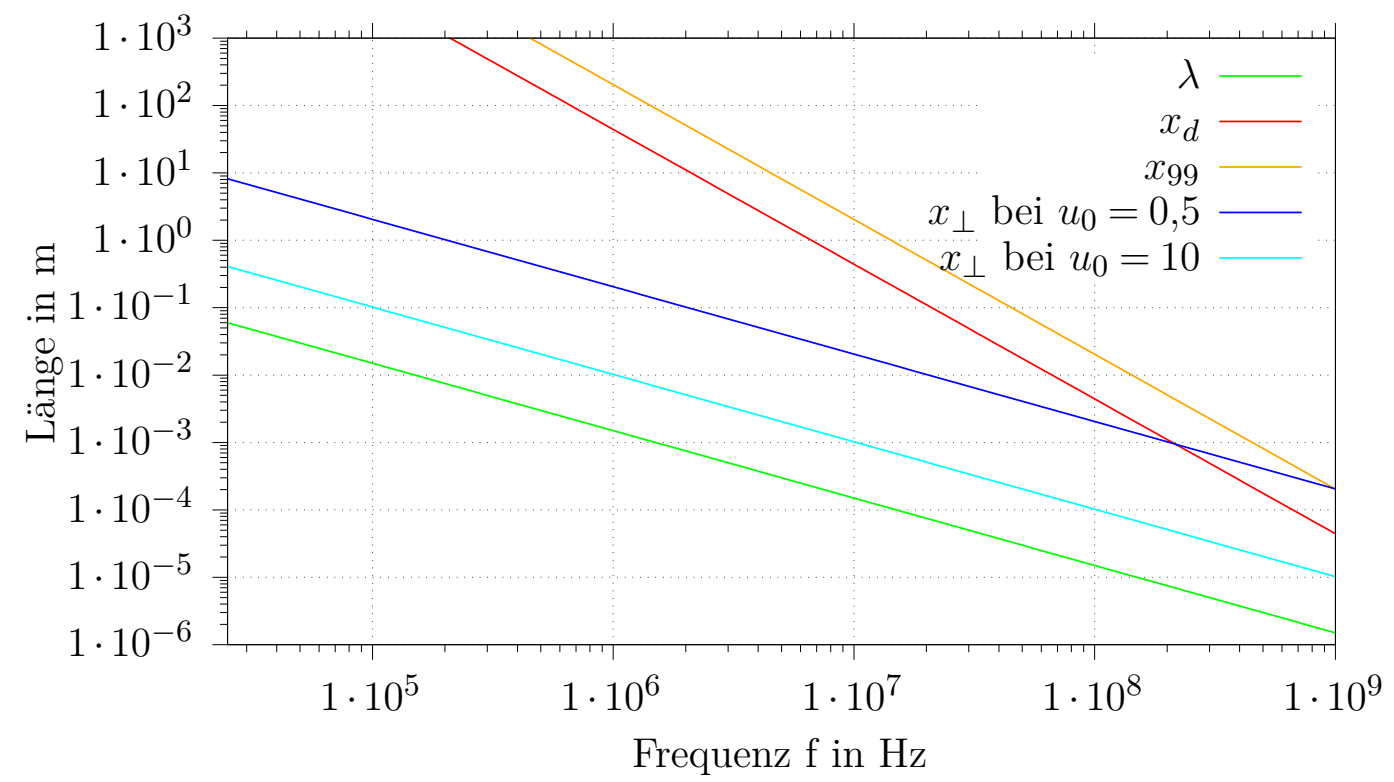

Abb. 2.2.: Doppelt logarithmischer Graph der Wellenlänge $\lambda$ sowie der Dissipationslänge $x_{d}$ und $x_{99}$ in Abhängigkeit von der Frequenz. Zum Vergleich ist noch die Schockdistanz $x_{\perp}$ aus Abschnitt 2.6.4 für zwei Amplituden $u_{0}=0,5 \mathrm{~m} / \mathrm{s}$ und $u_{0}=10 \mathrm{~m} / \mathrm{s}$ dargestellt.

Neben der üblichen Länge $x_{d}$, auf der die Intensität auf den Bruchteil $\frac{1}{e}$ gesunken ist, wird zusätzlich die Länge $x_{99}$ dargestellt, bei der nur noch ein Prozent der Anfangsintensität vorhanden ist.

Für die in dieser Arbeit relevanten Frequenzen zwischen $500 \mathrm{MHz}$ bis $1000 \mathrm{MHz}$ liegt die Dissipationslänge in Wasser bei $20^{\circ} \mathrm{C}$ zwischen $x_{d} \approx 177 \mu \mathrm{m}$ bis $44 \mu \mathrm{m}$ bzw. $x_{99} \approx 815 \mu \mathrm{m}$ bis $204 \mu \mathrm{m}$, wenn nichtlineare Effekte vernachlässigt werden.

Wie auch aus Abbildung 2.2 entnommen werden kann, wird die Dissipationslänge mit zunehmender Frequenz kürzer, und zwar proportional zu $1 / f^{2}$. 
Neben der Dissipationslänge ist auch die Schockdistanz $x_{\perp}$ aus Abschnitt2.6.4 abgebildet. Bei den Parametern, die im experimentellen Teil dieser Arbeit verwendet werden $\left(f \approx 750 \mathrm{MHz}\right.$ und Werte von $\left.u_{0}>1 \mathrm{~m} / \mathrm{s}\right)$, lässt sich bereits aus der Abbildung ablesen, dass die Schockdistanz in derselben Größenordnung wie die Dissipationslänge liegt. Wie im Abschnitt 2.5.2 diskutiert wird, lässt sich das Schallfeld dann nicht durch eine einfach dissipierende Schallwelle darstellen.

Für die meisten anderen Flüssigkeiten (ausgenommen verflüssigte Gase wie $N_{2}, O_{2}$, u.a.) ist der Dämpfungskoeffizient größer als bei Wasser und damit die Dissipationslänge kürzer.

\subsection{Dimensionslose Kennzahlen}

Eine dimensionlose Kennzahl oder auch Kenngrößen werden zur Entdimensionierung von Gleichungen eingesetzt und helfen, Phänomene und Prozesse allgemeiner $\mathrm{zu}$ formulieren.

Im folgenden werden einige Kennzahlen vorgestellt.

\subsubsection{Hydrodynamische Reynoldszahl}

Die Reynoldszahl ist eine dimensionslose Kennzahl, die nach dem Physiker Osborne Reynolds benannt wurde. In der Regel wird mit der Reynoldszahl das Verhältnis von Trägheitskräften zu Zähigkeitskräften bezeichnet. Diese Kennzahl wird in dieser Arbeit als hydrodynamische Reynoldszahl $R e_{\text {hy }}$ bezeichnet:

$$
R e_{\mathrm{hy}} \equiv \frac{\rho v d}{\mu}
$$

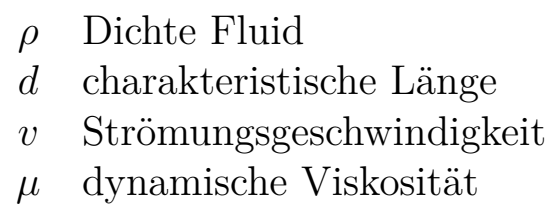

Bei großen Reynoldszahlen ist mit einer turbulenten Strömung zu rechnen. Bei einer Rohrströmung findet der Übergang von laminarer zur turbulenten Strömung bei $R e_{\text {hy }} \approx 2300$ statt [15].

\subsubsection{Akustische Reynoldszahl}

Ob bei der Ausbreitung einer akustischen Welle Nichtlinearitäten oder mehr die Dämpfung berücksichtigt werden müssen, wird durch die akustische Reynoldszahl $R e_{\mathrm{ak}}$ beschrieben werden. Nach Friend et al. [4] ist die Definition 
von Naugolnykh und Ostrovsky [16] am nützlichsten:

$$
R e_{\mathrm{ak}} \equiv \frac{\beta}{\alpha} \frac{u \omega}{c_{0}^{2}}=\frac{1}{\alpha \cdot x_{\perp}}
$$

Dabei steht $\alpha$ für den Dämpfungskoeffizienten, und $\beta$ (der Koeffizient der Nichtlinearität) ist aus der Zustandsgleichung (2.1.11) bekannt. Die Kreisfrequenz $\omega$ und die Schallgeschwindigkeit $c_{0}$ sind die weiteren nötigen Parameter für die akustische Reynoldszahl.

Diese Definiton der akustischen Reynoldszahl wird auch als Gol'dberg Zahl bezeichnet [6]. Die dimensionslose Zahl setzt die Stärke der Nichtlinearität ins Verhältnis zur Dämpfung, bzw. stellt sie das Produkt aus der Dämpfung $\alpha$ (siehe Abschnitt 2.4.1) und der später in der nichtlinearen Akustik erläuterten Schockdistanz $x_{\perp}=c_{0}^{2} /(\beta u \omega)$ (aus Abschnitt 2.6.4) dar.

Damit ist $R e_{\mathrm{ak}}$ ein Maß, ob die Nichtlinearität oder die Dämpfung überwiegt. Für $R e_{\mathrm{ak}}<1$ überwiegt die Dämpfung bzw. für $R e_{\mathrm{ak}}>1$ sind die nichtlinearen Effekte entscheidend.

Bei noch deutlicheren Quotienten für $R e_{\mathrm{ak}} \ll 1$ verhindert die Dissipation der Schallenergie im Fluid, dass sich die nichtlineare Effekte entwickeln.

Wenn $R e_{\mathrm{ak}} \gg 1$ akkumulieren sich nichtlinearen Effekte und die Wellenausbreitung muss mit einer entsprechenden Gleichung, z.B. der Burgers-Gleichung, berechnet werden. Die anfänglich sinusförmige Welle entwickelt sich zum Sägezahn bzw. zur Stoßwelle.

Mit den im experimentellen Teil der Arbeit verwendeten Parametern aus Tabelle 2.4 ergibt sich

$$
R e_{\mathrm{ak}}=1 \rightarrow u_{0}=\frac{\alpha c_{0}^{2}}{\beta \omega} \approx 1,54 \mathrm{~m} / \mathrm{s}
$$

\begin{tabular}{|c|c|c|}
\hline$\alpha$ & $\approx 226 \cdot 10^{-16} \mathrm{~s}^{2} / \mathrm{m} \cdot f^{2}$ & akustischer Dämpfungskoeffizient, siehe 2.4 .1 \\
\hline$\beta$ & $\approx 3,5$ & Koeffizient der Nichtlinearität \\
\hline$u_{0}$ & & Schallschnelle \\
\hline$f$ & $=750 \mathrm{Mhz}$ & Frequenz \\
\hline$c_{0}$ & $=1480 \mathrm{~m} / \mathrm{s}$ & Schallgeschwindigkeit \\
\hline
\end{tabular}

Tab. 2.4.: Parameter (von Wasser) für die Gleichung (2.5.3)

Daher sollten in Wasser bei einer Frequenz von $f=750 \mathrm{MHz}$ ab Schallschnellen von $u_{0} \approx 1,54 \mathrm{~m} / \mathrm{s}$ die Nichtlinearitäten nicht vernachlässigt werden. 


\subsubsection{Machzahl}

Die Machzahl ist eine dimensionslose Kennzahl, mit der die Strömungsgeschwindigkeit im Verhältnis zur Schallgeschwindigkeit angegeben wird:

$$
M a=\frac{v}{c_{0}}
$$

Bei der akustischen Machzahl wird statt der Strömungsgeschwindigkeit die Schallschnelle des Fluides in Relation zur Schallgeschwindigkeit gesetzt.

Erst bei Machzahlen über 0,3 muss man in der Regel Kompressibilitätseffekte berücksichtigen.

Mit den für diese Arbeit relevanten Größen wird eine Machzahl von $M a=$ 0,015 nicht überschritten, da keine Geschwindigkeiten $v>20 \mathrm{~m} / \mathrm{s}$ in Wasser $\left(c_{0}=1480 \mathrm{~m} / \mathrm{s}\right)$ beobachtet werden.

Jedoch sagt die Machzahl damit nur aus, dass auf Grund von hydrodynamischen Effekten keine Kompressibilitätseffekte zu erwarten sind. Über akustische Eigenschaften wird dabei keine Aussage getroffen.

\subsection{Nichtlineare Akustik}

Wie im Abschnitt 2.5.2 gezeigt wurde, ist es bei den Frequenzen und Amplituden, die im experimentellen Teil verwendet werden, teilweise nötig, Nichtlinearitäten zu berücksichtigen.

In diesem Abschnitt werden die nichtlinearen akustischen Gleichungen nach Westervelt und nach Burgers verglichen. Dabei wird die Schockdistanz eingeführt und im Zusammenhang mit der Dissipationslänge diskutiert.

\subsubsection{Westervelt-Gleichung}

Hamilton und Blackstock folgend [2] kann man mit der Konitnuitätsgleichung (2.2.5) und der Impulsgleichung (2.2.6) in der 2. Ordnung sowie der TaitGleichung als Zustandsgleichung folgende Wellengleichung 2. Ordnung herleiten:

$$
\square^{2} p+\frac{\delta}{c_{0}^{4}} \frac{\partial^{3} p}{\partial t^{3}}=-\frac{\beta}{\rho_{0} c_{0}^{4}} \frac{\partial^{2} p^{2}}{\partial t^{2}}-\left(\nabla^{2}+\frac{1}{c_{0}^{2}} \frac{\partial^{2}}{\partial t^{2}}\right) \mathcal{L}
$$

mit

$$
\begin{aligned}
\square & =\nabla^{2}-c_{0}^{-2}\left(\partial^{2} / \partial t^{2}\right) & & \text { d'Alembertscher Operator } \\
\delta & =\frac{1}{\rho_{0}}\left(\frac{4 \mu}{3}+\eta\right)+\frac{\kappa}{\rho_{0}}\left(\frac{1}{c_{v}}-\frac{1}{c_{p}}\right) & & \text { Diffusität des Schalls } \\
\mathcal{L} & =\frac{1}{2} \rho_{0} u^{2}-\frac{p^{2}}{2 \rho_{0} c_{0}^{2}} & & \text { zweite Ordnung Lagrangsche Dichte }
\end{aligned}
$$


Wenn man bei der Wellengleichung (2.6.1) den Term mit der Lagrangschen Dichte $\mathcal{L}$ vernachlässigt, erhält man die Westervelt-Gleichung (2.6.2] [2]. Dies ist möglich, da bei einer ebenen fortschreitenden Welle in der Näherung zweiter Ordnung $\mathcal{L}=0$ ist.

$$
\frac{1}{\rho c^{2}} \frac{\partial^{2} p}{\partial t^{2}}-\nabla \cdot\left(\frac{1}{\rho}\left(\nabla p+\frac{\delta}{c^{2}} \frac{\partial(\nabla p)}{\partial t}\right)\right)=\frac{\beta}{\rho^{2} c^{4}} \frac{\partial^{2} p^{2}}{\partial t^{2}}
$$

Diese Gleichung wird für numerische Simulationen eines nichtlinearen Schallfeldes in Abschnitt 3.3 genutzt.

\subsubsection{Burgers-Gleichung}

Die Westervelt Gleichung (2.6.2) kann durch die Annahme sich nur vorwärts ausbreitender Wellen vereinfacht werden und nimmt durch die Wahl geeigneter Koordinaten eine eindimensionale Form an. Entsprechend beschreibt die Burgers-Gleichung (2.6.3) die Ausbreitung einer ebenen akustischen Welle in einem Fluid unter Berücksichtigung der Dämpfung und der Nichtlinearität [2]:

$$
\frac{\partial u}{\partial x}-\frac{\beta}{c_{0}^{2}} u \frac{\partial u}{\partial \tau}=\frac{\delta}{2 c_{0}^{3}} \frac{\partial^{2} u}{\partial \tau^{2}}
$$

mit

$$
\begin{array}{ll}
\delta=\frac{1}{\rho_{0}}\left(\frac{4 \mu}{3}+\eta\right)+\frac{\kappa}{\rho_{0}}\left(\frac{1}{c_{v}}-\frac{1}{c_{p}}\right) & \text { Diffusität des Schalls } \\
\tau=t-\frac{x}{c_{0}} & \text { Verzögerungszeit } \\
\beta & \text { Koeffizient der Nichtlinearität }
\end{array}
$$

wie von Burgers [17] beschrieben. Die Welle wird durch einen schwingenden Kolben an der Stelle $x=0$ erzeugt und es wird das amplitudenabhängige, nichtlineare Verhalten der Welle berücksichtigt.

Durch Einführen der folgenden dimensionslosen Größen, wie in [18] gezeigt, kann die Gleichung 2.6.3) in die meist benutzte dimensionslose Form überführt werden.

$$
\begin{array}{lll}
U=u / u_{0} & \begin{array}{l}
\text { Die Geschwindigkeit } u \text { wird durch Anregungsgeschwindigkeit } \\
u_{0} \text { normiert }
\end{array} \\
\theta=\omega \tau & \begin{array}{l}
\text { Die Zeit } \tau \text { wird durch die Kreisfrequenz } \omega \\
\text { entdimensionalisiert }
\end{array} \\
\sigma=\frac{\beta \omega u_{0} x}{c_{0}^{2}} & \begin{array}{l}
\text { Für eine dimensionslose räumliche Koordinate wird } x \text { durch } \\
\text { die Schockdistanz } x_{\perp} \text { geteilt, siehe Abschnitt 2.6.4 }
\end{array} \\
\Gamma=\frac{1}{R e_{\mathrm{ak}}} & \begin{array}{l}
\text { Als Maß für die Nichtlinerarität wird die inverse } \\
\text { akutische Reynoldszahl } R e_{\text {ak }} \text { aus Abschnitt 2.5.2 verwendet }
\end{array}
\end{array}
$$




\section{Theorie}

Somit ergibt sich:

$$
\frac{\partial U}{\partial \sigma}-U \frac{\partial U}{\partial \theta}=\Gamma \frac{\partial^{2} U}{\partial \theta^{2}}
$$

Für die dimensionslose Gleichung (2.6.4) gibt es eine analytische Lösung, auf die in Abschnitt 2.6.7 weiter eingegangen wird.

\subsubsection{Fubini-Lösung}

Eine analytische Lösung der Burgers-Gleichung für die Ausbreitung von nichtlinearen Wellen bei Vernachlässigung der Dämpfung wurde von Fubini [19] gefunden. Nach [20, 21, 22, 23] und wie von [6] zusammengefasst lässt sich die Fubini-Lösung wie folgend Darstellen.

Die Fubini-Lösung ist für ebene, periodische, laufende Wellen mit endlicher Amplitude in der Nähe der Quelle bis zur Schockdistanz gültig:

$$
u(x, t)=u_{0} \sum_{n=1}^{\infty} B_{n}(x) \sin \left(n \omega\left(t-\frac{x}{c}\right)\right)
$$

mit

$$
B_{n}(x)=\frac{2}{n \sigma} J_{n}(n \sigma)
$$

Dabei steht $J_{n}$ für die sphärische Besselfunktion erster Gattung und $\sigma=\frac{x}{x_{\perp}}$ ist 
die dimensionslose örtliche Koordinate, die durch Division des Ortes $x$ durch die Schockdistanz $x_{\perp}$ gebildet wird, siehe Absatz 2.6.4.

Die Fubini-Lösung ist nur bis zur Schockdistanz gültig und beschreibt eine Sinuskurve, die sich von einer Schallquelle ausbreitet und mit Annähern an die Schockdistanz sich zu einer Sägezahnfunktion entwickelt. Bei der Schockdistanz erreicht die Funktion eine senkrechte Tangente.

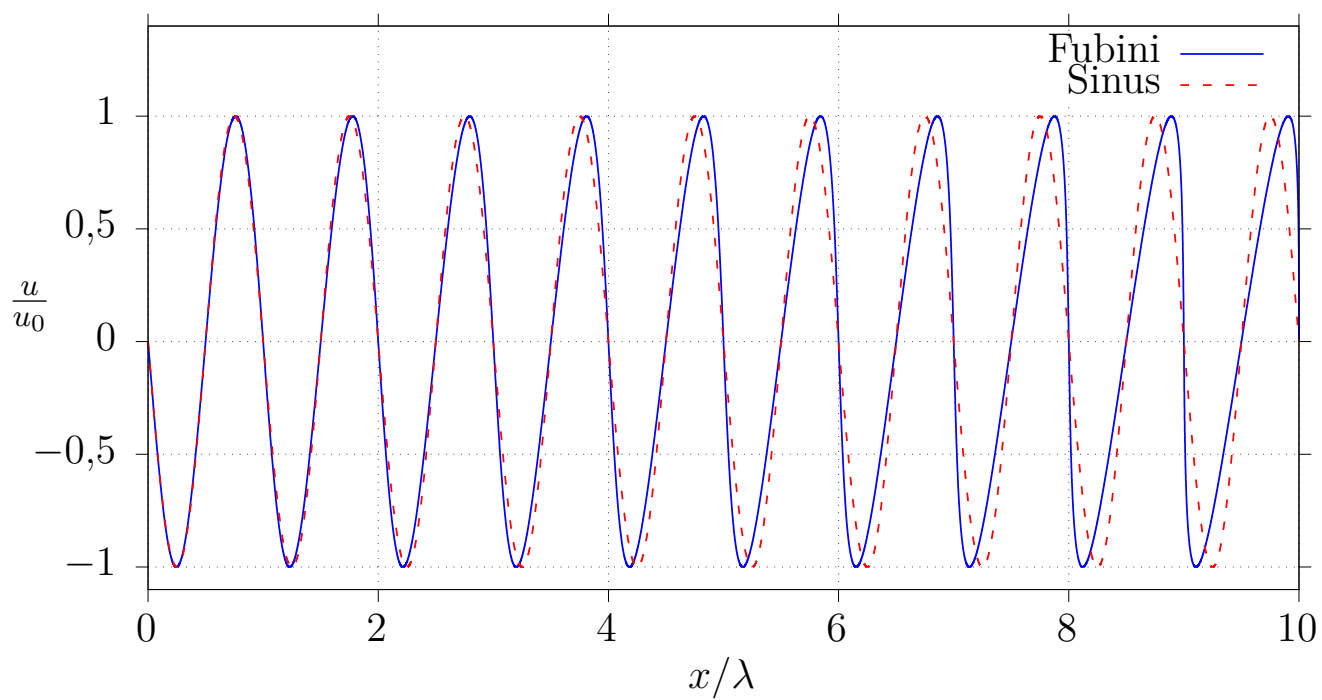

Abb. 2.3.: Fubini-Lösung mit der Schallquelle bei $x=0$ und Erreichen von $x_{\perp}$ bei $x=x_{\perp}=10 \lambda$. Gut zu erkennen ist der Übergang von der Sinuskurve zum Sägezahn. Zusätzlich ist als Vergleich eine einfache Sinuskurve dargestellt.

In der Abbildung 2.3 wurden die Parameter so gewählt, dass die Schockdistanz bereits nach 10 Wellenlängen erreicht ist. Damit lässt sich der Übergang vom Sinus zum Sägezahn in einer Abbildung darstellen, dies entspricht aber extremen Bedingungen.

Der Verlauf der Fubini-Lösung bei der Schockdistanz $x_{\perp}$ wird in Abbildung 2.4 noch mal genauer gezeigt. Hier werden die letzten 6 Wellenzügen vor der Schockdistanz dargestellt. Die Parameter sind so gewählt, dass $x_{\perp} \gg \lambda$ ist, also die Änderung pro Wellenlänge vernachlässigt werden kann. 


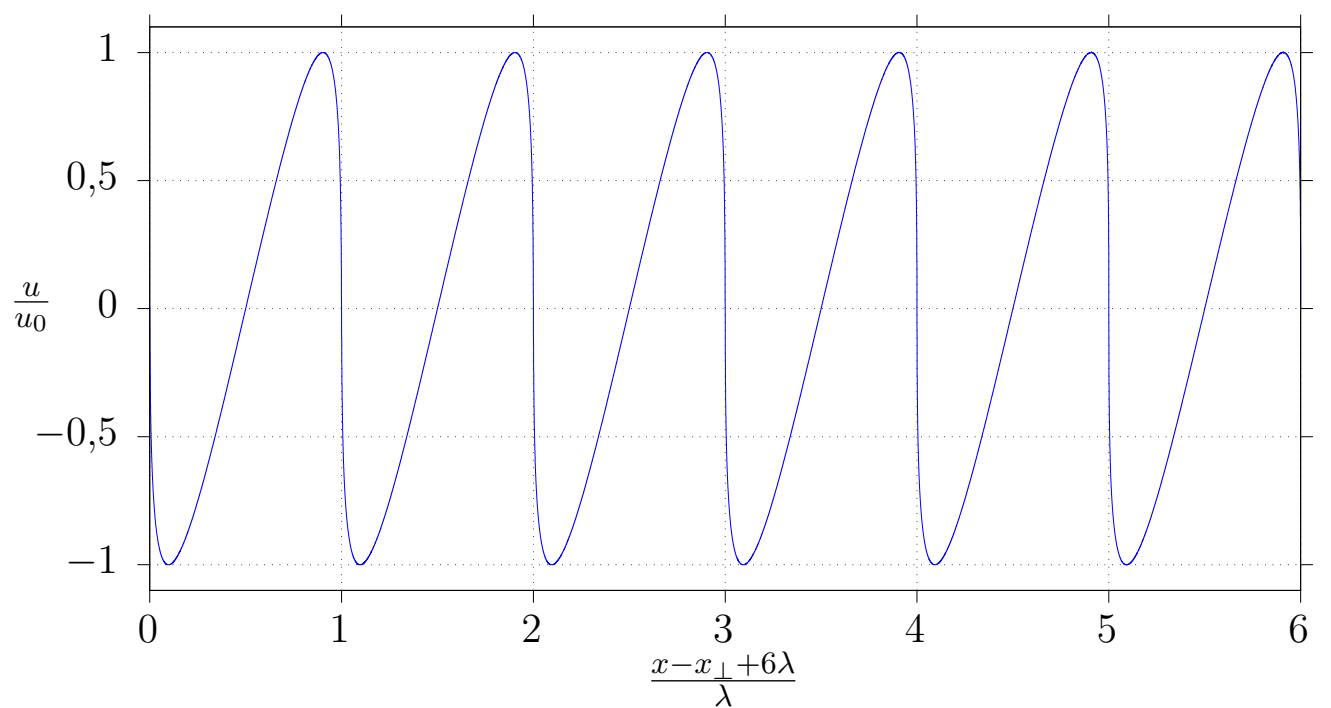

Abb. 2.4.: Die letzen 6 Perioden der Schallwelle vor der Schockdistanz $x_{\perp}$ zur Zeit $t_{\perp}$

Um die Betrachtung noch zu erweitern, wird in der Graphik 2.5 noch die Schallwelle in der zeitlichen Dimension betrachtet. Die Parameter wurden wie bei der Abbildung 2.4 gewählt.

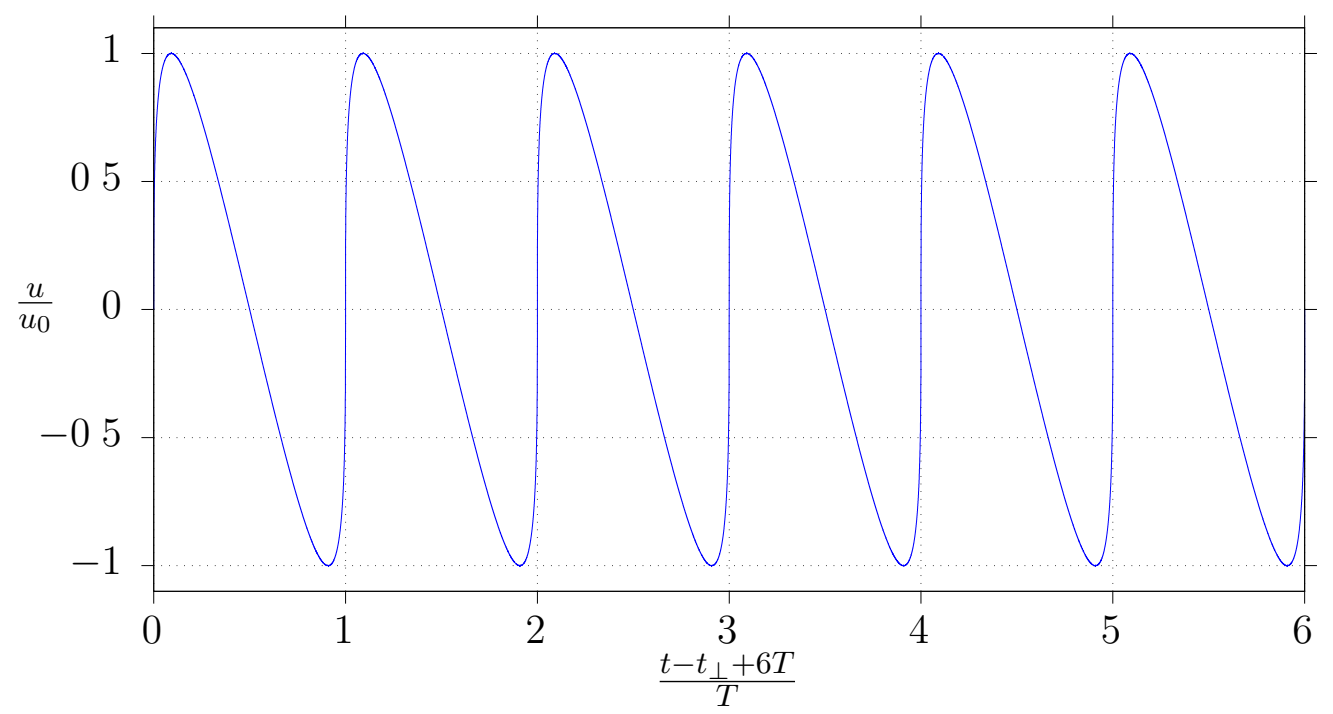

Abb. 2.5.: Die letzten 6 Perioden der Schallwelle vor der Schockzeit $t_{\perp}$ an der Stelle $x_{\perp}$

Das Aufsteilen der Welle kommt durch höherperiodische Komponenten, die bei der Gleichung (2.6.5) aufsummiert werden. Die ersten 8 Harmonischen sowie die Amplitude der fundamentalen Frequenz werden in Abbildung 2.6 gezeigt. Bei $x=0$ ist noch alle Energie bei der Fundamentalen $n=1$, wird 
aber mit zunehmender Entfernung von der Schallquelle auch auf die höheren Harmonischen verteilt.

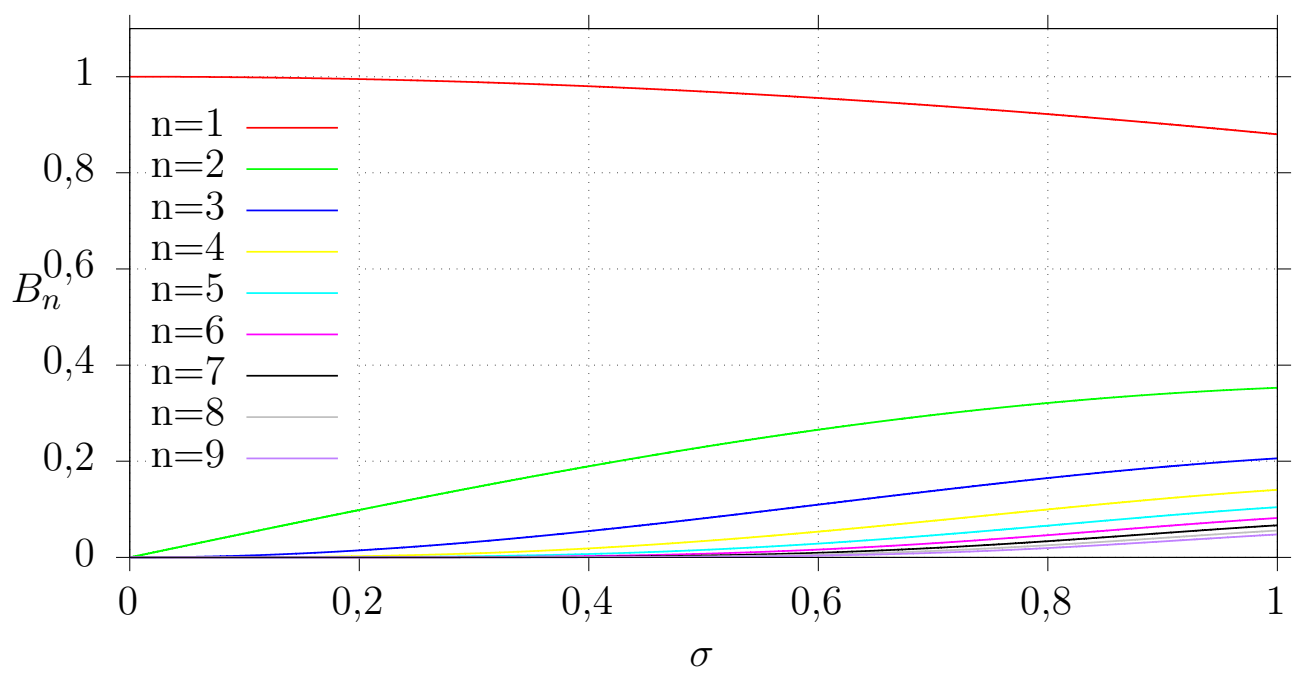

Abb. 2.6.: Amplitude der Frequenzen $B_{n}$ für $n=1$ bis $n=9$

Die analytische Fubini-Lösung kann ein Schallfeld von der Schallquelle bis zur Schockdistanz unter Vernachlässigung der Dämpfung der Schallwelle beschreiben.

Von Blackstock [24] werden noch folgende Erweiterungen an der Fubini-Lösung eingeführt, um die Dämpfung zu berücksichtigen.

$$
U(\sigma, \theta)=\sum_{n=1}^{\infty}\left[\frac{2 J_{n}(n \sigma)}{n \sigma}-\frac{2}{n R e_{\mathrm{ak}}} \sum_{r=1}^{\infty} r a_{r}\left(J_{n-r}(n \sigma)+J_{n+r}(n \sigma)\right)\right] \sin n \theta
$$

mit

$$
a_{r}=r\left(1-\sigma^{2}\right)^{-\frac{1}{2}} \sigma^{-r}\left[1-\left(1-\sigma^{2}\right)^{\frac{1}{2}}\right]^{r}
$$

Dabei ist der entscheidende neue Parameter $R e_{\mathrm{ak}}$ für die Intensität der Dissipation im Verhältniss zur Nichtlinearität verantwortlich (siehe Abschnitt 2.5.2).

Im Abschnitt 2.6.7 wird die erweiterte Fubini-Lösung 2.6.6 mit der BurgersGleichung, die die Dissipation berücksichtigt verglichen.

\subsubsection{Schockdistanz}

Die nichtlineare Fubini-Lösung aus Abschnitt 2.6.3 ist bis zur Schockdistanz gültig. Damit ist die Stelle gemeint, an der die Funktion des Schalldrucks beim 
Übergang von einer Sinuskurve zu einer Sägezahnfunktion eine vertikale Tangente bildet. Durch die Nichtlinearitäten werden höhere Frequenzen angeregt, die zu der Sägezahn- bzw. Stoßbildung führen. Wie in [6, 16] gezeigt, ist die Schockdistanz für nichtlineare Wellen ohne Dämpfung folgend definiert:

$$
x_{\perp}=\frac{1}{\beta M a k_{0}}=\frac{c_{0}^{2}}{\beta u_{0} \omega}
$$

Für Wasser bei $20^{\circ} \mathrm{C}$ und mit den Parametern aus Tabelle 2.5 ergeben sich die Werte aus der Grafik 2.7 .

Tab. 2.5.: Symbole und Parameter für die Schockdistanz, die für Abbildung 2.7 verwendet wurden

\begin{tabular}{cl}
\hline \hline$\beta=1+B / 2 A \approx 3,5$ & Koeffizient der Nichtlinearität, siehe Gleichung 2.1 .11 \\
$u_{0} \approx 0 \mathrm{~m} / \mathrm{s}$ bis $25 \mathrm{~m} / \mathrm{s}$ & Fluid Schallschnelle \\
$c_{0}=1480 \mathrm{~m} / \mathrm{s}$ & Schallgeschwindigkeit von Wasser \\
$\omega=2 \pi f$ & Kreisfrequenz
\end{tabular}

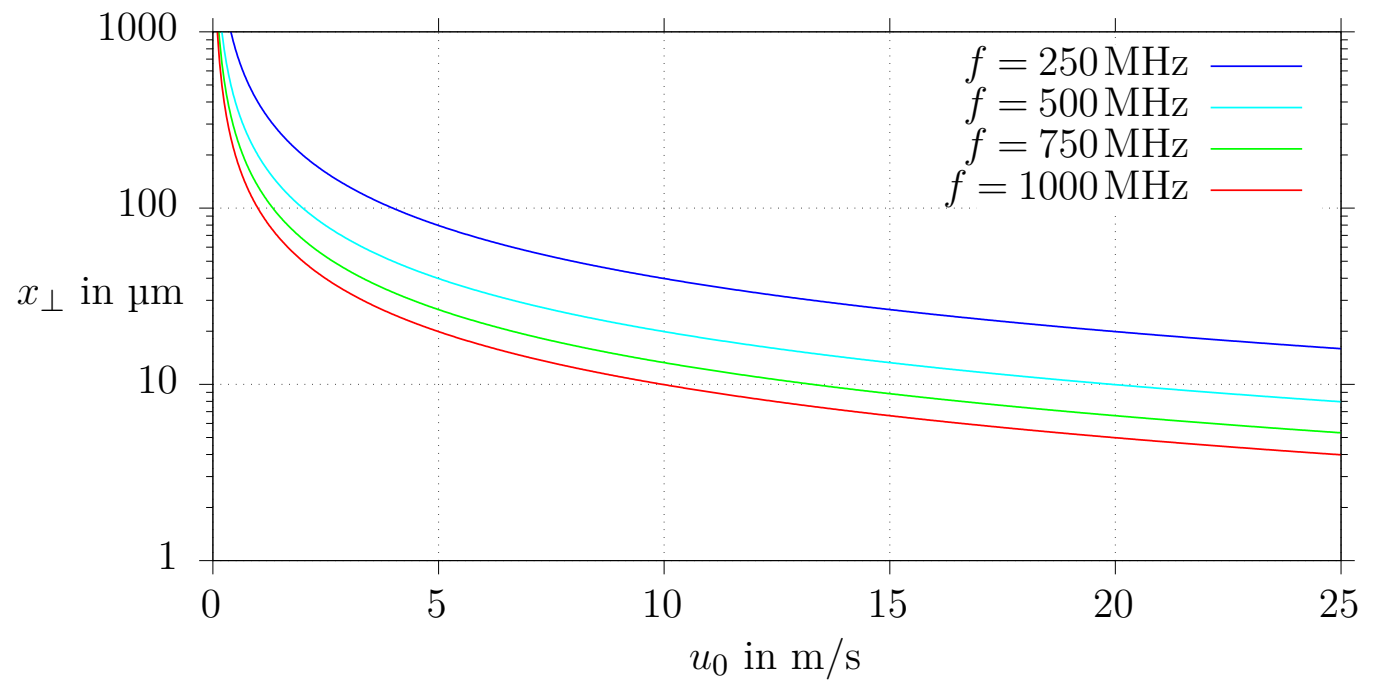

Abb. 2.7.: Schockdistanz $x_{\perp}$ in Abhänigkeit von der Partikelgeschwindigkeit $u_{0}$ für die Frequenzen $f \in[250,500,750,1000] \mathrm{MHz}$.

Bei $f=750 \mathrm{MHz}$ beträgt bereits bei einer Anregungspartikelgeschwindigkeit von $u_{0}=1 \mathrm{~m} / \mathrm{s}$ die Schockdistanz nur $x_{\perp 1} \approx 133 \mu \mathrm{m}$ und reduziert sich bei $u_{0}=10 \mathrm{~m} / \mathrm{s}$ auf $x_{\perp 10} \approx 13 \mu \mathrm{m}$.

Aus der Schockdistanz $x_{\perp}$ lässt sich mit der Schallgeschwindigkeit die Schockformationszeit $t_{\perp}$ berechnen.

$$
t_{\perp}=\frac{x_{\perp}}{c_{0}}=\frac{1}{\beta u_{0} \omega}
$$


Diese Zeit gibt an, wie lange eine Welle durch das nichtlineare Medium läuft, bis sich (ohne Dämpfung) eine Stoßwelle ausbildet.

Passend zum oberen Beispiel für $f=750 \mathrm{MHz}$ ergibt sich für $u_{0}=1 \mathrm{~m} / \mathrm{s} \Rightarrow$ $t_{\perp 1} \approx 90 \mathrm{~ns}$ bzw. für $u_{0}=10 \mathrm{~m} / \mathrm{s} \Rightarrow t_{\perp 10} \approx 9 \mathrm{~ns}$.

\subsubsection{Fay-Lösung}

Da auch der weitere Verlauf der Schallwelle nach $x_{\perp}$ von Interesse ist, wird hier die Fay-Lösung betrachtet. Die Fay-Lösung ist eine analytische Lösung der nichtlinearen Wellengleichung 2. Ordnung 2.6.1) und wurde erstmals 1931 von Fay [25] gezeigt.

Die Fay-Lösung gilt wie die Fubini-Lösung für ebene, laufende, periodische Schallwellen mit endlicher Amplitude. Im Gegensatz zu der Fubini-Lösung ist die Fay-Lösung für eine weit entfernte Schallquelle $\sigma \gg 1$ gültig aber bereits ab $\sigma \approx 4$ ist der Fehler recht klein.

In der Form wie in Gleichung 2.6.9 wird sie von [26] verwendet und ist eine Verbesserung der ursprünglichen Lösung von Fay:

$$
\frac{u}{u_{0}}=\sum_{n=1}^{\infty} \frac{2 R e_{\mathrm{ak}}}{\sinh \left[n(1+\sigma) R e_{\mathrm{ak}}\right]} \sin \left[n \omega\left(t-\frac{x}{c_{0}}\right)\right]
$$

Tab. 2.6.: Symbole und Parameter für die Fay-Lösung, die für Abbildung 2.8 verwendet wurden

\begin{tabular}{|c|c|c|}
\hline$\overline{f f}$ & $=750 \mathrm{MHz}$ & Fundamentale Frequenz \\
\hline$u_{0}$ & $=10 \mathrm{~m} / \mathrm{s}$ & Anregung Partikelgeschwindigkeit \\
\hline$R e_{\mathrm{ak}}$ & $=6,39$ & akustische Reynoldszahl, siehe 2.5.2 \\
\hline $\begin{array}{l}\sigma \\
n\end{array}$ & $=x / x_{\perp}$ & $\begin{array}{l}\text { Normierte Ortskoordinate } \\
\text { n-te Harmonische }\end{array}$ \\
\hline
\end{tabular}

Eine detaillierte Herleitung und einen Beweis, dass die Fay-Gleichung eine asymptotische Lösung der Burgers-Gleichung ist, kann man in [27] finden.

Die Abbildungen 2.8 zeigt die Schallwelle nach Gleichung (2.6.9) und 2.9 zeigt die Amplitude. 


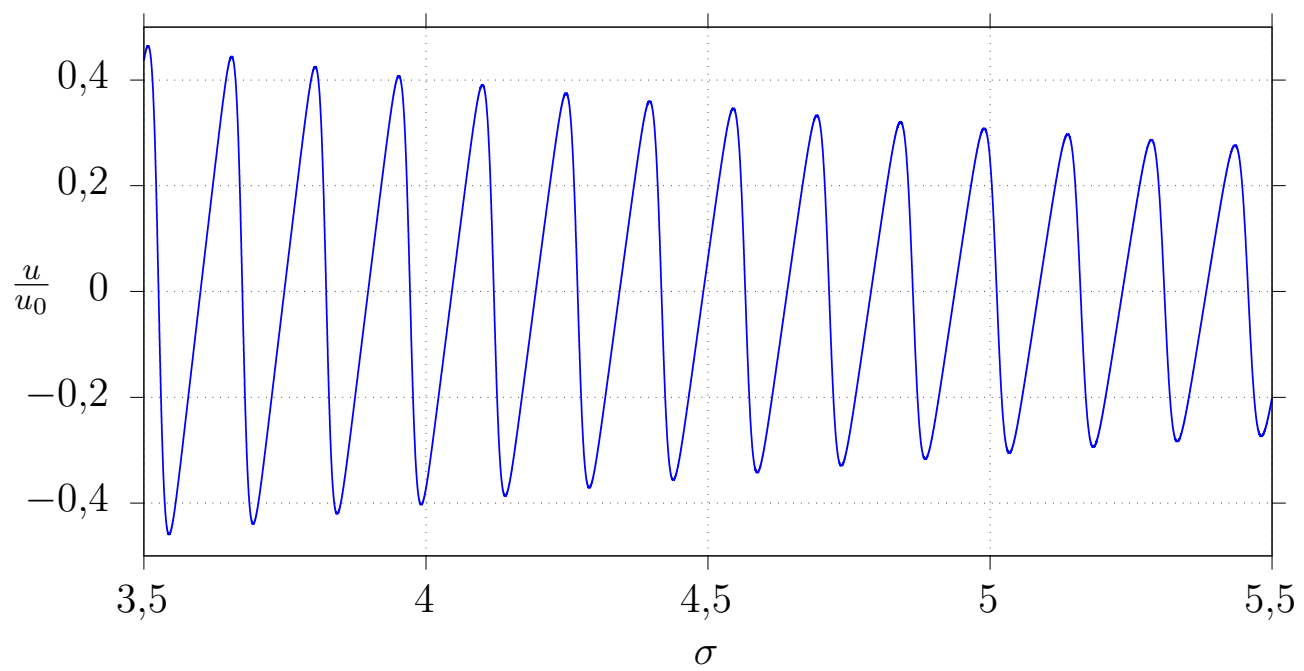

Abb. 2.8.: Fay-Lösung nach Gleichung 2.6 .9 mit den Parametern aus Tabelle (2.6). Man beachte hier $\sigma \gtrsim 4$. Die Aufsteilung geht mit größeren Laufstrecken $\sigma$ wieder zurück.

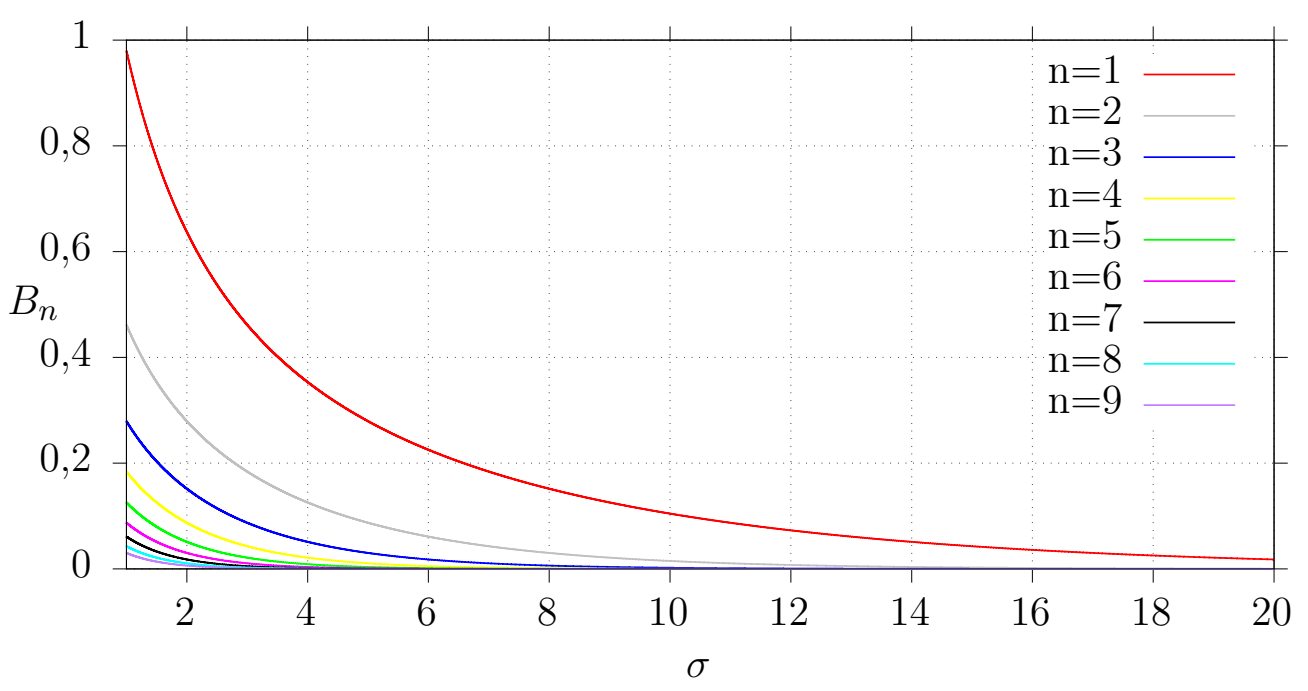

Abb. 2.9.: Amplitude der Fundamentalen sowie der Harmonischen bis $n=9$ nach Gleichung $(2.6 .9)$ ohne den Sinusterm.

Durch Grenzwertbetrachtungen [27] lassen sich folgende Aussage über die Funktion machen, wozu für eine einfachere Schreibweise an dieser Stelle die inverse akustische Reynoldszahl $\Gamma=1 / R e_{\text {ak }}$ eingeführt wird.

Mit $\Gamma \rightarrow \infty$ ergibt sich das verlustfreie Limit, welches eine Fourier-Reihe einer Sägezahnfunktion ist. 
Für $\sigma \gg 1$ und $\Gamma<\infty$ kann die Gleichung 2.6.9) durch

$$
\frac{u}{u_{0}} \approx 4 \Gamma e^{-\Gamma \sigma} \sin \left[n \omega\left(t-\frac{x}{c_{0}}\right)\right]
$$

angenähert werden, wenn man die höheren Harmonischen vernachlässigt, da diese stärker gedämpft werden. Dies bedeutet, dass für große Werte von $\sigma$ sich die Welle wieder einer Sinusform annähert.

Wenn man die höheren Harmonischen bei $\sigma \gg 1$ dennoch mitnimmt, ergibt sich

$$
\frac{u}{u_{0}}=4 \Gamma \sum_{n=1}^{\infty} e^{-n \Gamma \sigma} \sin \left[n \omega\left(t-\frac{x}{c_{0}}\right)\right]
$$

und durch Einsetzen von $\sigma$ und $\Gamma$ in Gleichung (2.6.11) ergibt sich

$$
u=4 \frac{b \omega}{2 \beta \rho_{0} c_{0}} \sum_{n=1}^{\infty} e^{\frac{-n b \omega^{2} x}{2 \rho_{0} c_{0}^{3}}} \sin \left[n \omega\left(t-\frac{x}{c_{0}}\right)\right] .
$$

Hier kann man erkennen, dass für den asymptotischen Grenzfall die Amplitude nicht von der Anfangsamplitude $u_{0}$ abhängt. Dieses Phänomen wird als Saturation bezeichnet. Ein nichtlineares Fluid dämpft umso stärker, je größere die Amplitude ist, womit nach einer gewissen Laufzeit der Welle durch das Medium die verbleibende Amplitude nicht von der anfänglichen Amplitude abhängt.

Ebenfalls kann man aus Gleichung (2.6.11) die Dämpfung der Amplitude ablesen. Die Dämpfung folgt $e^{-n \Gamma \sigma}$ und nicht wie aus der linearen Theorie zu erwarten $e^{-n^{2} \Gamma \sigma}$, also quadratisch mit der Frequenz ansteigend. Der Unterschied liegt in der Ursache für die höheren Harmonischen. In der linearen Theorie müssen diese durch die Randbedingung eingebracht werden, während bei der nichtlinearen Theorie die Harmonischen durch die Nichtlinearität generiert werden. Für die Fundamentale $n=1=n^{2}$ gibt es keinen Unterschied zwischen linearer und nichtlinearer Theorie.

\subsubsection{Verbindung der Fay- und der Fubini-Lösung}

Da sowohl die Fay- als auch die Fubini-Lösung in der Übergangsregion von nahe der Quelle zu einer weit entfernten Quelle nicht gültig sind, wurde an einer Verbindung der beiden Gleichungen gearbeitet, um die Lücke zu schließen. In [26] wird eine Verbindung der beiden Gleichungen gezeigt, die diesen Übergangsbereich von der Schockdistanz bis zur 4-fachen Schockdistanz beschreibt. Ab einer Entfernung von $\frac{1}{\alpha}$ wird allerdings diese Lösung auch wieder ungenau, denn die Dämpfung wird hier wieder vernachlässigt. 


\section{Theorie}

Die zusammengesetzte Lösung ist

$$
\frac{u}{u_{0}}=\sum_{n=1}^{\infty} B_{n}
$$

dabei ist die Amplitude der $n$-ten harmonischen durch

$$
B_{n}=\underbrace{\frac{2}{n \pi} V_{b}}_{\text {Sägezahn }}+\underbrace{\frac{2}{n \pi \sigma} \int_{\phi_{\min }}^{\pi} \cos [n(\phi-\sigma \sin \phi)] \mathrm{d} \phi}_{\text {Fubini }}
$$

gegen mit

$$
V_{b}=\frac{1}{\sigma} j_{0}^{-1}\left(\frac{1}{\sigma}\right) H(\sigma-1)
$$

dabei ist $H$ die Heaviside-Funktion und $j_{0}^{-1}\left(\frac{1}{\sigma}\right)$ berechnet die Größe, deren sphärische Besselfunktion 0-ter Ordnung gleich $1 / \sigma$ ist.

Die untere Grenze des Integrals ist durch $\phi_{\min }=\sigma V_{b}(\sigma)$ gegeben.

Gleichung (2.6.13) beschreibt die Amplitude der einzelnen Frequenzen, aus der sich die akustische Welle zusammensetzt. Bei $\sigma=0$ ist die gesamte Energie noch in der Fundamentalen wie in Abbildung 2.10 dargestellt.

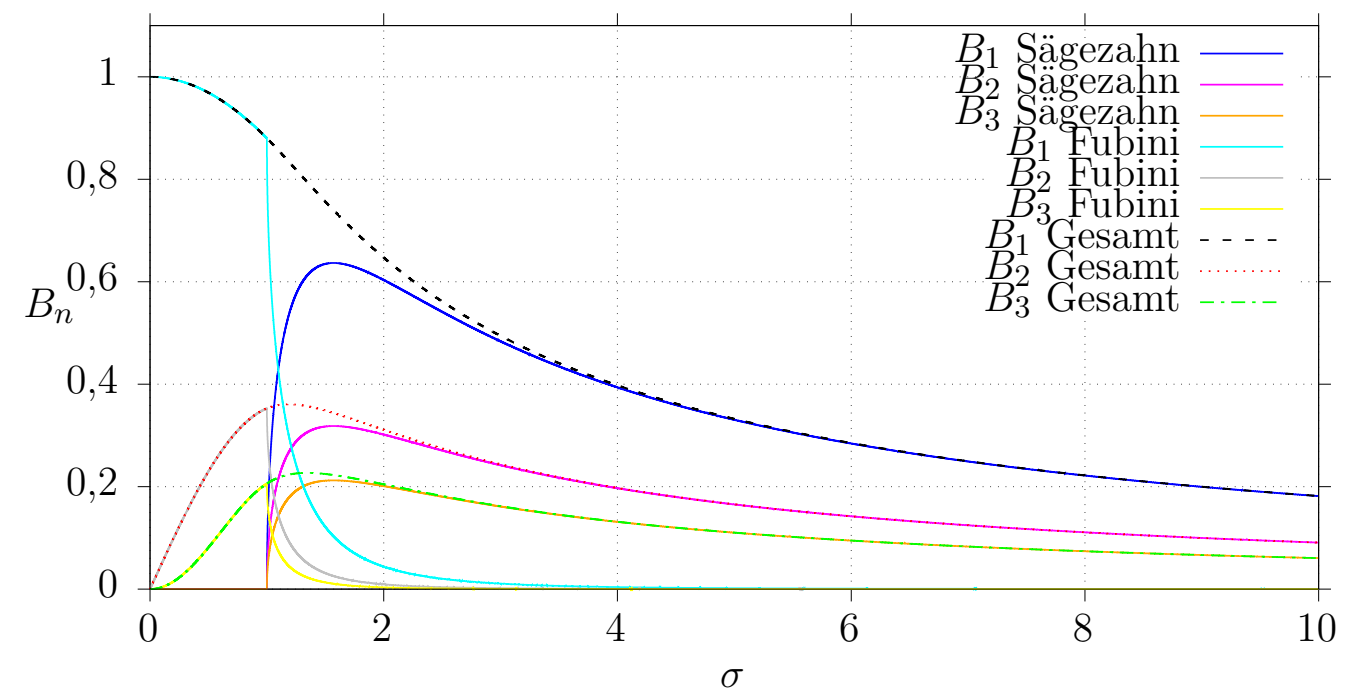

Abb. 2.10.: Verbindung der Fubini-Lösung mit der Fay-Lösung

Durch die Verbindung der Fay- und der Fubini-Lösung kann eine Welle von der Quelle bis weit entfernt von der Quelle berechnet werden, wobei die Entwicklung vom Sinus zum Sägezahn und wieder zurück zum Sinus beobachtet werden kann. 
Ein Problem bei der Fubini-Lösung ist, dass hier die Dämpfung nicht berücksichtigt wird. Sowohl für die Fay- als auch die Fubini-Lösungen gibt es Erweiterungen, die den Einfluss der Dämpfung berücksichtigen. Jedoch wird hier auf das Verbinden dieser Lösungen verzichtet.

Für den Parameterbereich dieser Arbeit würde ein Vernachlässigen der Dämpfung zu größeren Abweichungen führen, womit diese von Blackstock [20] beschriebene Lösung ungeeignet ist.

Wie in [27] werden im nächsten Abschnitt 2.6.7 die Fay-, die Fubini-Lösung und deren Verbindung durch Blackstock mit einer exakten Lösung der BurgersGleichung verglichen.

\subsubsection{Vergleich der Lösung der vollständigen Burgers-Gleichung mit Fay und Fubini}

Eine analytische Lösung der vollständigen ,viskosen“ Burgers-Gleichung (2.6.4) wird im Folgenden verwendet und mit den oben gezeigten Lösungen von Fay, Fubini (mit Dämpfung) 2.6.6 sowie deren Verbindung durch Blackstock (ohne Dämpfung) (2.6.13) verglichen.

Eine Lösung der Gleichung (2.6.4) ist mit der Cole-Hopf [28] [29] Transformation

$$
U=2 \Gamma \frac{\frac{\partial V}{\partial \theta}}{V}=2 \Gamma \frac{\partial}{\partial \theta}(\ln V)
$$

möglich, wobei V eine Lösung der Wärmeleitungsgleichung darstellt:

$$
\frac{\partial V}{\partial \sigma}=\Gamma \frac{\partial^{2} V}{\partial \theta^{2}}
$$

Die Lösung V kann auch wie folgend als Fourier-Reihe geschrieben werden:

$$
V(\sigma, \theta)=\sum_{k=0}^{\infty} A_{k} e^{-\Gamma k^{2} \sigma} \cos k \theta
$$


Mit der Randbedingung

$$
u(0, \tau)=u_{0} \sin \omega \tau
$$

für den Kolben an der Stelle $x=0$ ergibt sich für dimensionslose Formulierung

$$
U(0, \theta)=\sin \theta
$$

mit der die Gleichung gelöst werden muss.

Wie bereits von [27] durchgeführt, kann aus (2.6.14) und (2.6.16) sowie der Lösung von (2.6.4) mit (2.6.17) die folgende Lösung gefunden werden: [28, 30]

$$
U(\sigma, \theta)=\frac{-4 \Gamma\left[\sum_{n=1}^{\infty} e^{-n^{2} \Gamma \sigma} n(-1)^{n} I_{n}\left(\frac{1}{2 \Gamma}\right) \sin n \theta\right]}{\left[I_{0}\left(\frac{1}{2 \Gamma}\right)+2 \sum_{n=1}^{\infty} e^{-n^{2} \Gamma \sigma}(-1)^{n} I_{n}\left(\frac{1}{2 \Gamma}\right) \cos n \theta\right]}
$$

Dabei steht $I_{n}$ für die modifizierte Besselfunktion erster Gattung und $n$-ter Ordnung. Die Lösung (2.6.18) kann numerisch ausgewertet werden wie in der Abbildung 2.11 gezeigt. Die Abbildung zeigt die Amplitude der Fundamentalen für unterschiedlich starke Dämpfungen. Die Achsen sind durch die Anregungsintensität $u_{0}$ bzw. die Schockdistanz $x_{\perp}$ entdimensionalisiert. Zusätzlich zur exakten Lösung der Burgers-Gleichung ist noch im Bereich von 0 bis $\sigma$ die um die Dämpfung erweiterte Fubini-Lösung Gl. (2.6.6) und im Bereich von $3<\sigma<20$ die Fay-Lösung mit Dämpfung Gl. 2.6.12) dargestellt.

Die Blackstock-Lösung aus Gleichung (2.6.13) stellt den Grenzfall dar, in dem die Dissipation vernachlässigt wird und Nichtlinearitäten dominieren. Die Burgers-Gleichung nähert sich dieser Lösung mit $\Gamma \rightarrow 0$ an.

Die Fubini-Lösung aus Gleichung (2.6.6) ist für kleine $\Gamma$ eine gute Näherung, aber nur im Bereich von $\sigma \in[0 ; 1]$.

Für größere Werte von $\sigma$ ist die Fay-Lösung aus Gleichung (2.6.11) eine gute Näherung und gibt vor allem die Steigung gut wieder, ist aber etwas zur exakten Lösung verschoben.

Die "lineare" Lösung aus Gleichung 2.4.2) ist der Grenzfall, in dem die Nichtlinearität vernachlässigt wird und nur die Dissipation betrachtet wird. Wie zu erwarten, ist das eine gute Näherung für große $\Gamma$. Die dissipative Lösung hängt eigentlich nicht von $\Gamma$ ab, da aber die $x$-Achse durch $\sigma=\frac{x}{x_{\perp}}$ dargestellt wird, skaliert die Lösung mit $\Gamma$. 

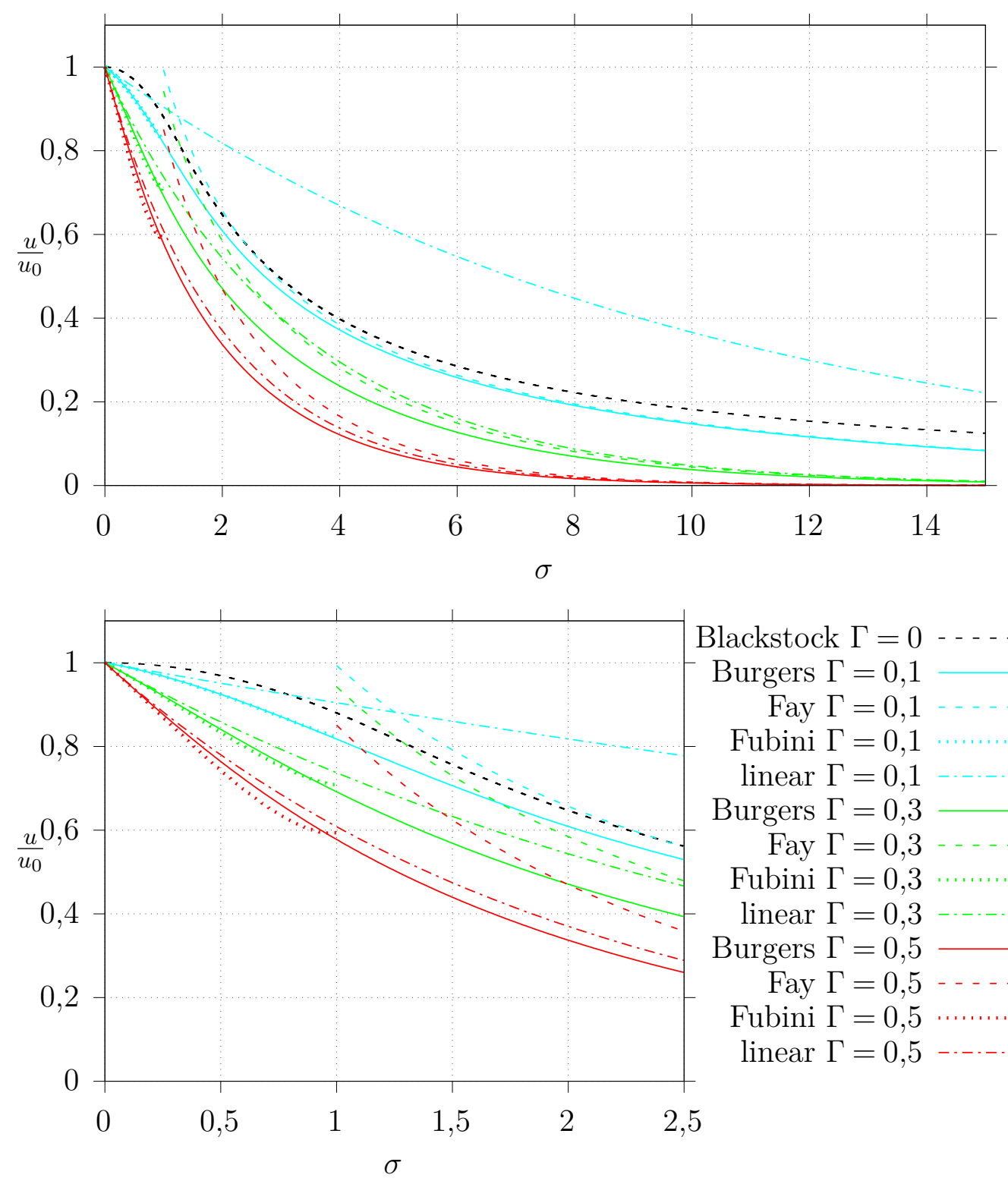

Blackstock $\Gamma=0$

Burgers $\Gamma=0,1$ Fay $\Gamma=0,1$ Fubini $\Gamma=0,1$ linear $\Gamma=0,1$ Burgers $\Gamma=0,3$ Fay $\Gamma=0,3$ Fubini $\Gamma=0,3$ linear $\Gamma=0,3$ Burgers $\Gamma=0,5$ Fay $\Gamma=0,5$ Fubini $\Gamma=0,5$ linear $\Gamma=0,5$

Abb. 2.11.: Vergleich der Amplituden der Fundamentalen von der Lösung der Burgers-Gleichung mit der dissipativen Fay-, Fubini- und nichtdissipativen Blackstock-Gleichung für verschiedene $\Gamma=1 / R e_{\mathrm{ak}}$. Oben: im Bereich von $0 \sigma$ bis $15 \sigma$. Unten: Im Bereich von $0 \sigma$ bis $2,5 \sigma$

Für die erste Harmonische ergibt sich ein ähnliches Bild, wie in der folgenden Abbildung 2.12 gezeigt wird.

Mit steigendem $\Gamma$ nimmt die Bedeutung der Harmonischen ab. Die FubiniLösung wird recht schnell ungenau und stellt schon ab $\Gamma=0,3$ den Verlauf nicht mehr gut dar. 

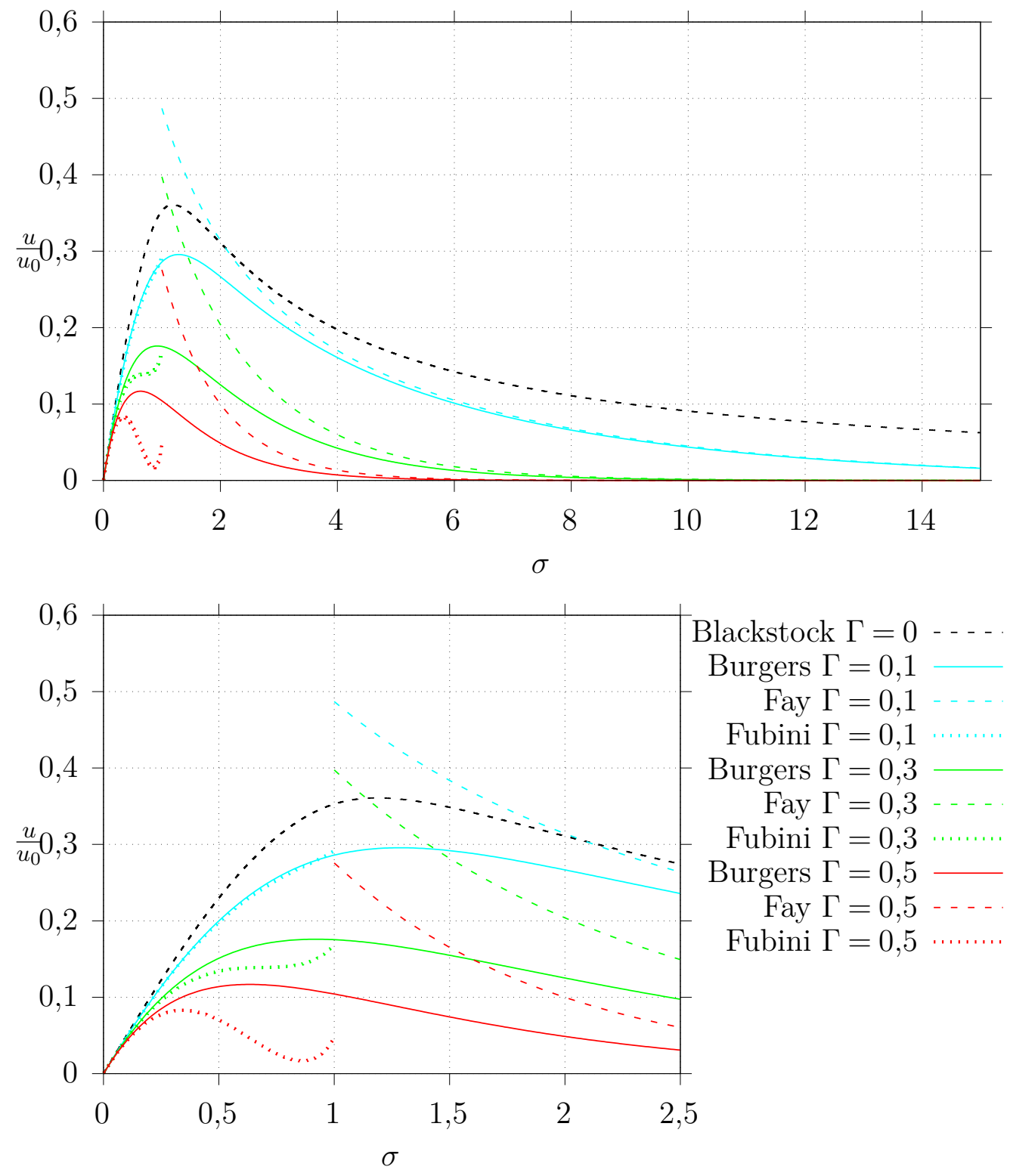

Abb. 2.12.: Die Amplitude der ersten Harmonischen der exakten Lösung der Burgers-Gleichung in Abhängigkeit von $\sigma$ für unterschiedliche $\Gamma$ im Vergleich zur dissipativen Fay-, Fubini- und nicht-dissipativen Blackstock-Gleichung. Oben: im Bereich von $0 \sigma$ bis $15 \sigma$. Unten: Im Bereich von $0 \sigma$ bis $2,5 \sigma$

Die einzige Gleichung, die für alle $\Gamma$ den Bereich von 0 bis einige $\sigma$ darstellen kann, ist die oben vorgestellte analytische Lösung der Burgers-Gleichung (2.6.18). Daher wird später bei der Berechnung der Volumenkraft einer nichtlinearen und ebenen Welle im Abschnitt 2.7.4 diese Lösung der BurgersGleichung verwendet. 


\subsection{Acoustic Streaming}

Unter der „akustischen Strömung“ (engl. ,acoustic streaming“) versteht man die gerichtete Bewegung eines Fluides, das sich durch Schallwellen in Bewegung setzt. Hierbei ist nicht die Vor- und Zurückbewegung durch die Schwingung gemeint, sondern eine im Mittel über Perioden des Schallfeldes gleichförmige Strömung. Diesen Effekt gibt es nur in verlustbehafteten Fluiden. Auch im deutschen Sprachgebrauch benutzt man oft das englische „acoustic streaming“. Hier wird nur die akustische Strömung die im Volumen des Fluids auftritt behandelt (nach Eckart und Zarembo). Daneben gibt es auch akustische Strömungen an Grenzflächen, wobei die viskose Grenzschicht eine Rolle spielt (Rayleigh- und Schlichting-Streaming). Für Details siehe [8, 31, 32].

\subsubsection{Eckart-Streaming (Langsame Strömung zweiter Ordnung)}

Die von Eckart beschriebene akustische Strömung [33] ist grundlegend und wird hier der Herleitung von [4] folgend gezeigt.

Wenn man bei der Kontinuitätsgleichung und der Impulsgleichung in der Näherung zweiter Ordnung nur die Komponenten zweiter Ordnung berücksichtigt, erhält man die Gleichungen (2.2.5) und (2.2.6), siehe Kapitel 2.

Die Zustandsgleichung 2.1.6 in der Näherung zweiter Ordnung ergibt

$$
p_{2}=A\left[\frac{\rho_{2}}{\rho_{0}}+\frac{1}{2} \frac{B}{A}\left(\frac{\rho_{1}}{\rho_{0}}\right)^{2}\right]=\frac{c_{0}^{2}}{\rho_{0}}\left[\frac{1}{2} \frac{B}{A} \rho_{1}^{2}+\rho_{0} \rho_{2}\right] .
$$

Da die Komponenten zweiter Ordnung der Energiegleichung nicht von den Gleichungen 2.2.5) und 2.2.6 abhängen, kann die Energiegleichung für die weitere Analyse vernachlässigt werden.

Durch Mittelung der Gleichungen (2.2.5, 2.2.6) und (2.7.1) über die Zeit ergeben sich die Ausdrücke

$$
\begin{aligned}
\left\langle\rho_{1} \frac{\partial \vec{u}_{1}}{\partial t}\right\rangle+\rho_{0}\left\langle\left(\vec{u}_{1} \cdot \nabla\right) \vec{u}_{1}\right\rangle & =-\nabla p_{d c}+\mu \nabla^{2} u_{d c}+\left(\eta+\frac{\mu}{3}\right) \nabla \nabla \cdot \vec{u}_{d c} \\
\rho_{0}\left(\nabla \cdot \vec{u}_{d c}\right)+\nabla \cdot\left\langle\rho_{1} \vec{u}_{1}\right\rangle & =0 \\
p_{d c} & =\frac{1}{2} \frac{c_{0}^{2}}{\rho_{0}}\left[\frac{B}{A}\left\langle\rho_{1}^{2}\right\rangle\right]+2 \rho_{0} \rho_{d c}
\end{aligned}
$$

wobei $\langle a\rangle=1 / T \int_{0}^{T} a(t) d t$ für die Mittelung über eine Schallfeldperiode steht. Der Index $d c$ bezeichnet die gleichgerichtete Komponente der zugehörigen Größe zweiter Ordnung, die beim Mitteln über eine Schallfeldperiode übrig bleibt. Zusammen mit der nullten Ordnung ergibt die $d c$ Komponente den gesamten Gleichgewichtszustand z.B. $\rho_{0}+\rho_{d c}$. 
Durch Kombinieren von (2.7.2) und 2.7.4) und Gruppieren der Relationen erster und zweiter Ordnung sowie unter Verwendung der Gleichung

$$
p_{1}=c_{0}^{2} \rho_{1}
$$

erhält man

$$
\vec{F}_{d c} \sim \nabla p_{d c}-\mu \nabla^{2} \vec{u}_{d c}
$$

Hier ist die Volumenkraftdichte durch

$$
\vec{F}_{d c}=-\frac{1}{c_{0}^{2}}\left\langle p_{1} \frac{\partial \vec{u}_{1}}{\partial t}\right\rangle-\rho_{0}\left\langle\left(\vec{u}_{1} \cdot \nabla\right) \vec{u}_{1}\right\rangle-\frac{1}{c_{0}^{2} \rho_{0}}\left(\eta+\frac{\mu}{3}\right) \nabla \nabla \cdot\left\langle p_{1} \vec{u}_{1}\right\rangle
$$

gegeben. Durch Verwenden der Strörungsrechnung ist diese Lösung limitiert auf kleine Strömungsgeschwindigkeiten. Die sogenannte akustische Strömungsgeschwindigkeit zweiter Ordnung $\vec{u}_{d c}$ muss deutlich kleiner sein als die akustische Schallschnelle $\vec{u}_{1}$.

\subsubsection{Akustische Strömung nach Zarembo}

Anders als bei der Methode der sukzessiven Annäherung bzw. der Störungsrechnung werden bei Zarembo [31] die Variablen $\rho, \vec{u}$ und $p$ aufgeteilt.

$$
\begin{aligned}
\vec{u} & =\vec{u}_{d c}(x, y, z)+\vec{u}_{A}(x, y, z, t) \\
p & =p_{d c}(x, y, z)+p_{A}(x, y, z, t) \\
\rho & =\rho_{d c}(x, y, z)+\rho_{A}(x, y, z, t)
\end{aligned}
$$

Wie auch in [4] erläutert, erfolgt die Aufteilung so, dass in der Komponente mit dem Index $d c$ alles beinhaltet ist, was bei einer Mittlung über eine, bzw. Vielfache einer Schallfeldperiode übrig bleibt. Am Beispiel der Geschwindigkeit $\vec{u}$ dargestellt, folgt

$$
\vec{u}_{d c}=\frac{1}{t_{2}-t_{1}} \int_{t_{1}}^{t_{2}} \vec{u} \mathrm{~d} t
$$

wobei für das Zeitintervall $t_{2}-t_{1}=n f^{-1}$ mit $n=1,2,3, \ldots$ gilt. Daraus folgt für die Komponenten mit dem Index ${ }_{A}$, die unter anderem die Akustik enthalten, dass die Mittelwerte $\left\langle\vec{u}_{A}\right\rangle=0,\left\langle p_{A}\right\rangle=0$ und $\left\langle\rho_{A}\right\rangle=0$ gleich Null sind.

Durch Einsetzen der Bedingungen aus (2.7.8) in die Navier-Stokes-Gleichung 2.1.2 und mit der Annahme, dass $\rho_{A} \ll \rho_{d c}$, erhält man folgende Impulsglei- 
chung:

$$
\frac{\partial \vec{u}_{d c}}{\partial t}+\left(\vec{u}_{d c} \cdot \nabla\right) \vec{u}_{d c}-\frac{\mu}{\rho_{d c}} \Delta \vec{u}_{d c}-\frac{1}{\rho_{d c}}\left(\eta+\frac{\mu}{3}\right) \nabla \nabla \cdot \vec{u}_{d c}=-\frac{1}{\rho_{d c}} \nabla p_{d c}+\vec{F}_{d c}
$$

mit

$\vec{F}_{d c}=-\rho_{d c}\left\langle\left(\vec{u}_{A} \cdot \nabla\right) \vec{u}_{A}\right\rangle+\frac{1}{\rho_{d c}}\left[\left\langle\rho_{A} \nabla p_{A}\right\rangle-\mu\left\langle\rho_{A} \Delta \vec{u}_{A}\right\rangle-\left(\eta+\frac{\mu}{3}\right)\left\langle\rho_{A} \nabla \nabla \cdot \overrightarrow{u_{A}}\right\rangle\right]$

Die stationäre Dichte $\rho_{d c}$ muss nicht mit der Dichte $\rho_{0}$ des ungestörten Fluides übereinstimmen, wie schon von Zarembo erläutert. Das Gleiche gilt für den stationären Druck $p_{d c}$, der von dem Druck $p_{0}$ abweichen kann.

Unter der Annahme, dass $\rho_{A} \ll \rho_{d c}$, was für kleine Machzahlen gerechtfertigt ist, lässt sich die Kraft aus Gleichung 2.7.10 mit der Näherung

$$
\frac{1}{\rho}=\frac{1}{\left(\rho_{d c}+\rho_{A}\right)} \frac{\rho_{d c}+\rho_{A}}{\left(\rho_{d c}+\rho_{A}\right)} \approx \frac{\rho_{d c}+\rho_{A}}{\rho_{d c}^{2}}
$$

weiter vereinfachen $\mathrm{zu}$

$$
\vec{F} \approx-\rho_{d c}\left\langle\left(\vec{u}_{d c} \cdot \nabla\right) \vec{u}_{d c}\right\rangle .
$$

Gerade in Flüssigkeiten wie Wasser ist die Annahme $\rho_{A} \ll \rho_{d c}$ auf Grund der geringen Kompressibilität gerechtfertigt. Die isentropische Kompressibilität von Wasser beträgt $\chi \approx 5 \cdot 10^{-10} \mathrm{~Pa}^{-1}$ [13]. Daraus ergibt sich sogar für eine hohe Partikelgeschwindigkeit von $u=20 \mathrm{~m} / \mathrm{s}$, die einem Druck von $p=\rho c u \approx$ $30 \mathrm{MPa}$ entspricht, ein Verhältnis von $\frac{\rho_{k}}{\rho_{0}} \approx 0,03$, also variiert die Dichte nur minimal mit dem Druck, wodurch die über die Zeit gemittelte Dichte $\rho_{d c}$ deutlich größer sein muss als die zeitliche variierende Dichte $\rho_{A}$.

Durch den hier verwendeten Ansatz ist die Fluidströmung nur über die zeitlich gemittelte Volumenkraft mit dem Schallfeld verknüpft. Dies wird in den Simulationen in Abschnitt 3 genutzt, indem zunächst das Schallfeld und die daraus resultierende Kraft berechnet wird. In einem weiteren Schritt wird dann die aus der Kraft resultierende Strömung berechnet.

\subsubsection{Volumenkraft einer dissipierenden ebenen Welle}

Im Folgenden wird die aus einer ebenen dissipierenden Schallwelle resultierende Volumenkraft berechnet. Dazu wird die Näherung der akustischen Volumenkraft $F$ aus Gleichung 2.7.11) verwendet.

Zunächst wird die Berechnung in kartesichen Koordinaten durchgeführt:

Unter der Annahme, dass die Partikelgeschwindigkeit $\vec{u}$ eine ebene sich in 
2. Theorie

z-Richtung ausbreitende und dissipierende Welle ist, die durch

$$
\vec{u}=\left(\begin{array}{c}
u_{x} \\
u_{y} \\
u_{z}
\end{array}\right)=\left(\begin{array}{c}
0 \\
0 \\
u_{0} \cdot \mathrm{e}^{-\alpha z} \sin \left(2 \pi f\left(\frac{z}{c}-t\right)\right)
\end{array}\right)
$$

beschrieben wird, folgt für die resultierende Kraft auf das Fluid:

$$
\begin{aligned}
\Rightarrow F & \approx-\rho_{0}\left\langle\left(u_{x} \frac{\partial}{\partial x}+u_{y} \frac{\partial}{\partial y}+u_{z} \frac{\partial}{\partial z}\right) \vec{u}\right\rangle \\
& =\rho_{0}-\left\langle u_{x} \frac{\partial \vec{u}}{\partial x}+u_{y} \frac{\partial \vec{u}}{\partial y}+u_{z} \frac{\partial \vec{u}}{\partial z}\right\rangle \\
& =\rho_{0}-\left\langle u_{z} \frac{\partial \vec{u}}{\partial z}\right\rangle
\end{aligned}
$$

Durch Einsetzen der Ausdrücke 2.7.12 und 2.7.14

$$
\frac{\partial \vec{u}}{\partial z}=-\frac{u_{0}}{c} \mathrm{e}^{-\alpha z}\left(\alpha c \sin \left(2 \pi f\left(\frac{z}{c}-t\right)\right)-\frac{2 \pi}{c} f \cos \left(2 \pi f\left(\frac{z}{c}-t\right)\right)\right)
$$

in Gleichung (2.7.13) folgt

$$
\begin{array}{r}
F \approx-\rho_{0}\left\langle u_{0} \cdot \mathrm{e}^{-\alpha z} \sin \left(2 \pi f\left(\frac{z}{c}-t\right)\right) .\right. \\
\left.-\frac{u_{0}}{c} \cdot \mathrm{e}^{-\alpha z}\left(\alpha c \sin \left(2 \pi f\left(\frac{z}{c}-t\right)\right)-\frac{2 \pi}{c} f \cos \left(2 \pi f\left(\frac{z}{c}-t\right)\right)\right)\right\rangle
\end{array}
$$

was sich in

$$
\begin{array}{r}
F \approx-\rho_{0}\left\langle-u_{0}^{2} \mathrm{e}^{-2 \alpha z} \sin \left(2 \pi f\left(\frac{z}{c}-t\right)\right) .\right. \\
\left.\left(\alpha \sin \left(\frac{2 \pi}{c} f\left(\frac{z}{c}-t\right)\right)-2 \pi f \cos \left(2 \pi f\left(\frac{z}{c}-t\right)\right)\right)\right\rangle
\end{array}
$$

umschreiben lässt. Durch weiteres Vereinfachen erhält man

$$
F \approx-\rho_{0}\left\langle-\frac{u_{0}^{2}}{c} \mathrm{e}^{-2 \alpha z} \alpha c \sin ^{2}\left(2 \pi f\left(\frac{z}{c}-t\right)\right)-\frac{\pi}{c} f \sin \left(4 \pi f\left(\frac{z}{c}-t\right)\right)\right\rangle
$$

aber für die Integration, die beim Mitteln ausgeführt wird, ist der Ausdruck

$$
F \approx-\rho_{0}\left\langle\frac{u_{0}^{2}}{2 c} \mathrm{e}^{-2 \alpha z}\left[\frac{\pi}{c} f \sin \left(4 \pi f\left(\frac{z}{c}-t\right)\right)+\alpha c\left(\cos \left(4 \pi f\left(\frac{z}{c}-t\right)\right)-1\right)\right]\right\rangle
$$

geeigneter. Um die Funktion über eine Schwingungsperiode z.B. $T=\frac{1}{f} \mathrm{zu}$ 
mitteln, muss

$F \approx-\rho_{0} f \cdot \int_{0}^{T} \frac{u_{0}^{2}}{2 c} \mathrm{e}^{-2 \alpha z}\left[2 \pi f \sin \left(4 \pi f\left(\frac{z}{c}-t\right)\right)+\frac{\alpha}{2}\left(\cos \left(4 \pi f\left(\frac{z}{c}-t\right)\right)-1\right)\right] d t$

berechnet werden. Durch Integrieren erhält man

$F \approx$

$$
-\rho_{0} f \cdot\left[\frac{u_{0}^{2} \mathrm{e}^{-2 \alpha z}\left(-\alpha c \sin \left(4 \pi f\left(\frac{z}{c}-t\right)\right)-2 \pi f \cos \left(4 \pi f\left(\frac{z}{c}-t\right)\right)-4 \pi \alpha c f t\right)}{8 \pi c f}\right]_{t=0}^{t=T}
$$

und da das Integral von $\sin (t)$ sowie $\cos (t)$ über eine Periode Null ist, lässt sich der Ausdruck zu

$$
\begin{aligned}
F & \approx-\rho_{0} f \cdot\left[\frac{-u_{0}^{2} \mathrm{e}^{-2 \alpha z} \cdot \alpha t}{2}\right]_{t=0}^{t=T}=-\rho_{0} f \cdot\left(\frac{-u_{0}^{2} \mathrm{e}^{-2 \alpha z} \cdot \alpha}{2 f}-0\right) \\
& =\rho_{0} \frac{\alpha u_{0}^{2} \mathrm{e}^{-2 \alpha z}}{2}
\end{aligned}
$$

vereinfachen. Das hier erhaltene Resultat

$$
F \approx \rho_{0} \frac{\alpha u_{0}^{2} \mathrm{e}^{-2 \alpha z}}{2}
$$

stimmt mit der in Moujed [13] angegebenen Lösung überein, wobei dort der Faktor $\frac{1}{2}$ in der Mittelung der ebenen Welle versteckt ist.

Abbildung 2.13 zeigt Gleichung 2.7.17) für eine $750 \mathrm{MHz}$ Welle in Wasser, welches einen Dissipationskoeffizienten von $\alpha \approx 12700 / \mathrm{m}$ hat. Man erkennt, dass die Volumenkraft bei dieser Frequenz bereits bei $z \approx 200 \mu \mathrm{m}$ fast vollständig abgeklungen ist. 


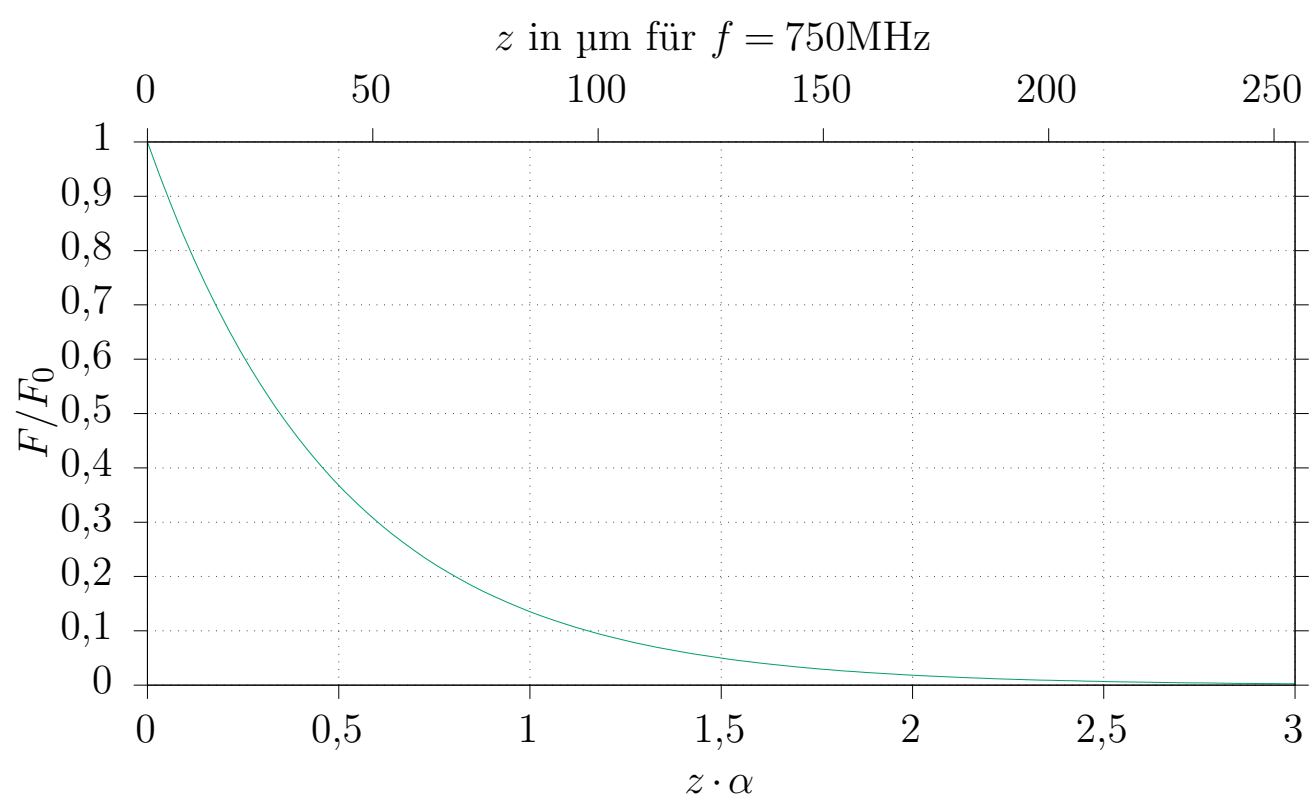

Abb. 2.13.: Volumenkraft einer ebenen in z-Richtung laufenden und dissipierenden Welle. Die y-Achse wurde durch die Kraft $F_{0}$ an der Stelle $z=0$ normiert. Die untere $z$-Achse wurde mit der Dämpfungslänge $\alpha$ normiert, womit sie frequenzunabhängig ist. Die obere $z$-Achse gilt für eine Frequenz $f=750 \mathrm{MHz}$.

\subsubsection{Volumenkraft einer dissipierenden nichtlinearen ebenen Welle}

Die Schallwelle wird in diesem Abschnitt mit der Burgers-Gleichung aus Abschnitt 2.6.7 berechnet, wodurch das nichtlineare Verhalten bei höheren Amplituden berücksichtigt wird.

Die Schallwelle wird vom Sinus zum Sägezahn durch das Anregen von höheren Frequenzen. Dies führt zu einem schnelleren Dissipieren der Welle und damit auch zu einer anderen Volumenkraft auf das Fluid. Die Volumenkraft wird mit der Gleichung 2.7.11 durch das Mitteln über einer Schallfeldperiode numerisch berechnet. 


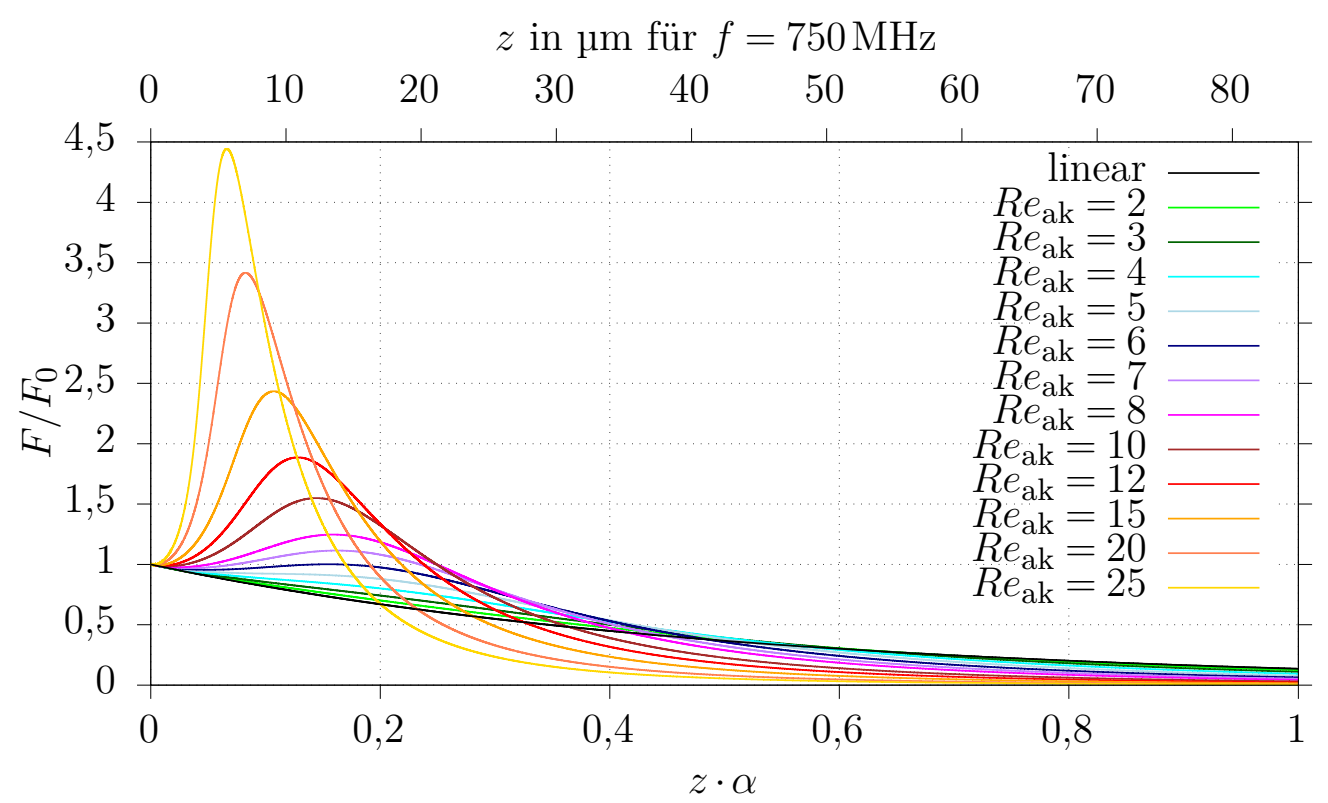

Abb. 2.14.: Volumenkraft einer ebenen in z-Richtung laufenden und nichtlinearen Welle nach der Burgers-Gleichung. Die y-Achse wurde durch die Kraft $F_{0}$ an der Stelle $z=0$ normiert. Die x-Achse wurde durch die Dämpfungslänge normiert. Die Intensität des Schallfeldes wurde durch $R e_{\mathrm{ak}}$ normiert.

Der Vorteil der dimensionslosen Achsen in Abbildung 2.14 ist, dass sie damit für alle Frequenzen gültig ist. Durch das Entdimensionieren der x-Achse mit $\alpha$ bleibt auch bei unterschiedlichen Amplituden die Skalierung der Achse gleich und ändert sich nur mit der Frequenz, was ein Vorteil gegenüber der Schockdistanz $\sigma$ ist, die sowohl von der Frequenz als auch von der Amplitude abhängt.

In Abhängigkeit von der Amplitude, die hier über $R e_{\text {ak }}$ aus Abschnitt 2.5.2 ebenfalls dimensionslos dargestellt ist, ist zu erkennen, dass sich die Dämpfungslänge bei großen Amplituden deutlich verkürzt. Ebenfalls entsteht ein Peak in der Volumenkraft zwischen $z \cdot \alpha \approx 0,05-0,2$.

Bei einer Frequenz von $f=750 \mathrm{MHz}$ sind ab einer Schallschnelle von $u_{0}=$ $1,5 \mathrm{~m} / \mathrm{s}$, was einem $R e_{\mathrm{ak}} \approx 1$ entspricht, die ersten Effekte der Nichtlinearität zu erkennen. Aus der Tabelle 2.7 kann man weitere Werte für $f=500 \mathrm{MHz}$, $750 \mathrm{MHz}$ und $1000 \mathrm{MHz}$ entnehmen. 


\begin{tabular}{c|c|c|c|c|c|c} 
für & \multicolumn{7}{|c}{$R e_{\mathrm{ak}}$} \\
$500 \mathrm{MHz}$ & 1 & 2 & 5 & 10 & 15 & 20 \\
\hline$u_{0}[\mathrm{~m} / \mathrm{s}]$ & 1,04 & 2,09 & 5,22 & 10,43 & 15,65 & 20,86 \\
\hline$F_{0}\left[\mathrm{~N} / \mathrm{cm}^{3}\right]$ & 2,8 & 11,4 & 71,1 & 284 & 640 & 1137 \\
\hline$x_{\perp}[\mu \mathrm{m}]$ & 191 & 95,5 & 38,2 & 19,1 & 12,37 & 9,54 \\
\hline
\end{tabular}

\begin{tabular}{c|c|c|c|c|c|c} 
für & \multicolumn{7}{|c}{$R e_{\mathrm{ak}}$} \\
$750 \mathrm{MHz}$ & 1 & 2 & 5 & 10 & 15 & 20 \\
\hline$u_{0}[\mathrm{~m} / \mathrm{s}]$ & 1,56 & 3,13 & 7,82 & 15,65 & 23,47 & 31,29 \\
\hline$F_{0}\left[\mathrm{~N} / \mathrm{cm}^{3}\right]$ & 14 & 58 & 360 & 1439 & 3238 & 5756 \\
\hline$x_{\perp}[\mu \mathrm{m}]$ & 84,89 & 42,44 & 16,98 & 8,49 & 5,66 & 4,24 \\
\hline
\end{tabular}

\begin{tabular}{c|c|c|c|c|c|c} 
für & \multicolumn{7}{|c}{$R e_{\mathrm{ak}}$} \\
$1000 \mathrm{MHz}$ & 1 & 2 & 5 & 10 & 15 & 20 \\
\hline$u_{0}[\mathrm{~m} / \mathrm{s}]$ & 2,09 & 4,17 & 10,43 & 20,86 & 31,29 & 41,72 \\
\hline$F_{0}\left[\mathrm{~N} / \mathrm{cm}^{3}\right]$ & 45 & 182 & 1137 & 4548 & 10234 & 18196 \\
\hline$x_{\perp}[\mathrm{\mu m}]$ & 47,75 & 23,87 & 9,55 & 4,77 & 3,18 & 2,39 \\
\hline
\end{tabular}

\begin{tabular}{c|c|c|c|c|c|c} 
ohne & \multicolumn{7}{|c}{$R e_{\mathrm{ak}}$} \\
Dimension & 1 & 2 & 5 & 10 & 15 & 20 \\
\hline$F_{\max } / F_{0}$ & 1 & 1 & 1 & 1,55 & 2,43 & 3,42 \\
\hline$x_{d e} \cdot \alpha$ & 0,99 & 0,96 & 0,81 & 0,61 & 0,49 & 0,42 \\
\hline$x_{\perp} \cdot \alpha$ & 1 & 0,5 & 0,2 & 0,1 & 0,07 & 0,05 \\
\hline
\end{tabular}

Tab. 2.7.: Numerisch berechnete Partikelgeschwindigkeit $u_{0}$, Volumenkraft $F_{0}$, Maximale Volumenkraft $F_{\max }$, Schockdistanz $x_{\perp}$ und effektive Dämpfungslänge $x_{d e}$ für $f \in[500,750,1000]$ und $R e_{\mathrm{ak}} \in$ $[1,2,5,10,15,20]$.

In der Tabelle 2.7 wird mit der effektiven Dämpfungslänge $x_{d e}$ die Strecke bezeichnet, bei der die nicht linear berechnete Volumenkraft auf $F_{0} / e^{2}$ gefallen ist, was demselben Dämpfungswert entspricht, die die linear berechnete Volumenkraft an der Stelle $1 / \alpha$ hat. 


\subsubsection{Skalierung und Proportionalitäten}

Wie schon von Moudjed [13] durchgeführt, lassen sich Proportionalitäten von der Intensität des Schallfeldes und der daraus resultierenden Strömung finden. Nahe der Schallquelle ist zu erwarten, dass die Trägheit entscheidend ist. Im Weiteren wird die Achse senkrecht und mittig über dem Schallwandler beachtet und nur die Größenordnungen verglichen.

Die Schallintensität $I_{a k}$ einer ebenen akustischen Welle kann mit

$$
I_{a k}=\overline{\rho c u^{2}}=\frac{1}{2} \rho c u_{0}^{2} e^{-2 \alpha z}
$$

abgeschätzt werden [34]. Hier ist das Abklingen gemäß linearer Dämpfung mit berücksichtigt.

Durch Einsetzen von Gleichung (2.7.18) in die Gleichung der Volumenkraft einer ebenen dissipierenden Welle (2.7.17) ergibt sich

$$
F=\frac{2 \alpha I_{a k}}{c} \text {. }
$$

Aus der Kontinuitätsgleichung (2.1.1) kann für eine stationäre Strömung, die nur in z-Richtung strömt, die folgende Relation zwischen Volumenkraft und Impulsstrom bestimmt werden:

$$
F=\rho \frac{\partial u_{z}^{2}}{\partial z}
$$

Durch Gleichsetzen der Gleichung (2.7.19) und 2.7.20 ergibt sich die Proportionalität

$$
\rho \frac{\partial u^{2}}{\partial z} \propto \frac{2 \alpha I_{a k}}{c}
$$

Die Leistung des Schallfeldes $P_{a k}$ ist durch das Integral

$$
P_{a k}=\int_{0}^{R_{\text {beam }}} I_{a k}(r) 2 \cdot \pi d r
$$

gegeben; mit $R_{\text {beam }}$ als Radius des Schallfeldes. Betrachtet man weiter nur die Größenordnung, kann man aus dem Integral folgende Proportionalität ablesen:

$$
I_{a k} \propto \frac{P_{a k}}{\pi R_{\text {beam }}^{2}}
$$

und durch Einsetzen in die Proportionalität (2.7.21) ergibt sich für die Strö- 
mungsgeschwindigkeit in $z$-Richtung

$$
u_{z} \propto \sqrt{\frac{\alpha}{\rho c} \frac{P_{a k}}{R_{\text {beam }}^{2}} z} .
$$

Die akustische Leistung ist proportional zur elektrischen Leistung am Schallwandler, die benötigt wird, um das Schallfeld zu erzeugen. Die elektrische Leistung $P_{e l}$ ist mit der angelegten Spannung $U_{e l}$ am Wandler über

$$
P_{e l}=\frac{U_{e l}^{2}}{R_{e l}}
$$

verknüpft, mit dem elektrischen Widerstand $R_{e l}$. Hierdurch ergibt sich für $u_{z}$ in Abhängigkeit von der elektrischen Spannung $U_{e l}$ die Gleichung

$$
u_{z} \propto \sqrt{\frac{\alpha}{\rho c} \frac{U_{e l}^{2}}{R_{\text {beam }}^{2} \cdot R_{e l}} z}
$$

Also ist ein linearer Zusammenhang zwischen der angelegten Spannung und der Strömungsgeschwindigkeit zu erwarten.

\subsection{Kavitation}

Unter Kavitation, aus dem Lateinischen cavitare „aushöhlen“, versteht man das Aufreißen einer Flüssigkeit durch Unterdruck. Dabei entstehen Blasen, die mit Dampf oder mit in der Flüssigkeit gelösten Gasen gefüllt sind.

Im Unterschied zum Sieden, wobei die Temperatur erhöht wird, geschieht die Blasenbildung aber in „kalten“ Flüssigkeiten durch das Wirken einer Zugspannung. Hierbei unterscheidet man zwischen hydrodynamischer und akustischer Kavitation.

Hydrodynamische Kavitation entsteht in Unterdruckzonen bei rasch strömenden Flüssigkeiten und kann zum Beispiel bei Schiffsschrauben oder Pumpen auftreten und zu deren Erosion führen.

Bei akustischer Kavitation wird die zum Aufreißen benötigte Zugspannung durch Druckunterschiede in akustischen Wellen erzielt. Dieser Effekt wird bei Ultraschallreinigungsbädern eingesetzt. Hier sind die Kaviationsblasen maßgeblich für den Reinigungseffekt verantwortlich.

Flüssigkeiten können außerdem auch durch lokalen Energieeintrag aufreißen, z.B. Laser-, Teilchenstrahlung sowie durch Blitzentladungen

\subsubsection{Keller-Miksis-Modell}

Unter der Annahme sphärischer Symmetrie kann man die Dynamik von Kavitationsblasen mit einer gewöhnlichen Differentialgleichung für den Radius 
$R$ darstellen.

Keller und Miksis [35] betrachten die Kompressibilität bis zur 1. Ordnung in der Mach-Zahl der Blasenwand $M=\frac{\dot{R}}{c}$. Sie entwickelten unter der Annahme einer rein sphärischen Oszillation und einer konstanten Anzahl an Gasmolekülen in der Blase folgende als Keller-Miksis-Gleichung bekannte Formel (2.8.1). Die Blase wird durch die Druckdifferenz $p$ zwischen Blasseninnendruck $p_{i}$ und Umgebungsdruck im Fluid um die Blase $p_{a}$, also $p=p_{i}-p_{a}$ getrieben. Ergänzt um den Dampfdruck und einer sinusförmigen Schallanregung folgt nach Lauterborn [36]:

$$
\left(1-\frac{\dot{R}}{c}\right) R \ddot{R}+\frac{3}{2} \dot{R}^{2}\left(1-\frac{\dot{R}}{3 c}\right)=\left(1+\frac{\dot{R}}{c}\right) \frac{p_{l}}{\rho}+\frac{R}{\rho c} \frac{\mathrm{d} p}{\mathrm{~d} t}
$$

mit

$$
p=\left(p_{\text {stat }}-p_{v}+\frac{2 \sigma_{O}}{R_{n}}\right)\left(\frac{R_{n}}{R}\right)^{3 \eta}-p_{\text {stat }}+p_{v}-\frac{2 \sigma_{O}}{R}-\frac{4 \mu}{R} \dot{R}-p_{A} \sin (\omega t)
$$

c Schallgeschwindigkeit in der Flüssigkeit

$p_{v} \quad$ Dampfdruck über dem Fluid

$\sigma_{O} \quad$ Oberflächenspannung

Die Keller-Miksis-Gleichung lässt sich mit akzeptablem Rechenaufwand, selbst bei hohen Schalldruckamplituden und daraus resultierenden intensiven Kollapsen, numerisch lösen und liefert dabei gute Resultate.

\subsubsection{Linearisierung des Keller-Miksis-Modells}

Für Blasen, deren Ruheradius $R_{n}$ die Bedingung $4 \mu / \rho c \ll R_{n} \ll c / \omega=\lambda / 2 \pi$ erfüllt und deren Anregungsdruck deutlich kleiner als der Umgebungsdruck $p_{A} \ll p_{\text {stat }}$ ist, gibt es die Möglichkeit, das Keller-Miskis-Modells mit dem Ansatz $R(t)=R_{n}+R^{\prime}(t)$ zu linearisieren. Wie z.B. von Parlitz [37] gezeigt wurde, ergibt sich für die Linearisierung

$$
\ddot{R}^{\prime}+\alpha_{L} \dot{R}^{\prime}+\omega_{0}^{2} R^{\prime}=-\frac{p_{A}}{\rho R_{n}} \cos (\omega t)
$$

mit

$$
\omega_{0}^{2}=\frac{1}{\rho R_{n}^{2}}\left[3 \eta p_{\text {stat }}+\frac{2 \sigma_{O}}{R_{n}}(3 \eta-1)\right], \quad \alpha_{L}=\frac{4 \mu}{\rho R_{n}^{2}}+\frac{w_{0}^{2} R_{n}}{c} .
$$

Gleichung (2.8.3) kann zum Bestimmen der linearen Resonanzfrequenz $f_{\text {res }}=$ $\omega_{0} /(2 \pi)$ bei bekanntem Blasenruheradius $R_{n}$ verwendet werden oder umgekehrt zur Bestimmung des Resonanzradius bei bekannter Frequenz. Der li- 


\section{Theorie}

neare Resonanzradius wurde erstmals von Minnaert [38] bestimmt, weswegen man den Resonanzradius auch als Minnaert-Radius bezeichnet. In Abbildung 2.15 ist der Minneart-Radius für Wasser und bei Normaldruck nach Gleichung (2.8.3) im Vergleich zur einfachen Faustregel für die Resonanzfrequenz $f_{\text {res }}=\frac{3 \mathrm{~m} / \mathrm{s}}{R_{n}}$ dargestellt.

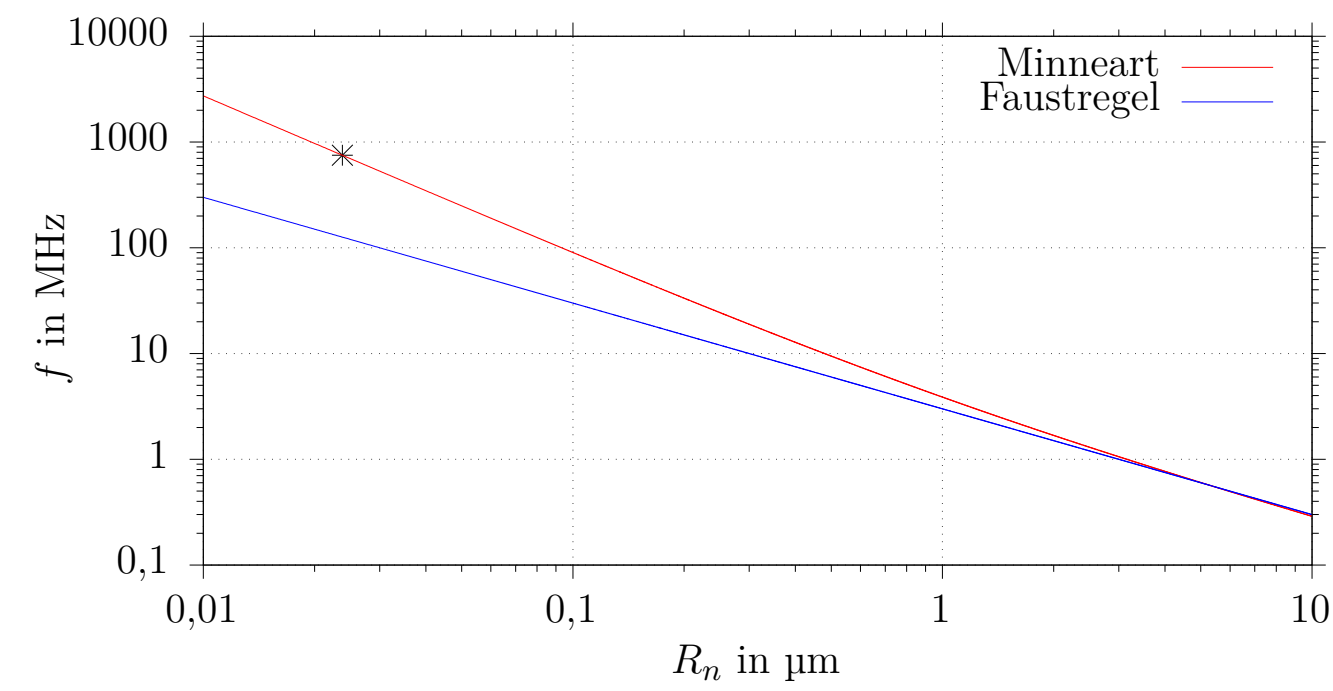

Abb. 2.15.: Die Grafik zeigt die Resonanzferquenz $f_{\text {res }}$ in Abhängigkeit vom Blasenradius für Wasser bei Normaldruck. Einmal nach Gleichung 2.8.3 (rot) und nach der Faustregel $f_{\text {res }}=\frac{3 \mathrm{~m} / \mathrm{s}}{R_{n}}$ (blau).

Gerade für die hohen Frequenzen dieser Arbeit ist die Faustformel sehr ungenau, da die Oberflächenspannung nicht eingeht. Wie man in der Abbildung 2.15 erkennen kann, haben Blasen mit einem Ruheradius von $R_{n}=23,78 \mathrm{~nm}$ ihre Resonanz bei $750 \mathrm{MHZ}$. 


\subsubsection{Blake-Schwelle}

Bei der Blake-Schwelle wird zwischen der statischen und der dynamischen Schwelle unterschieden. Durch statische Betrachtungen folgt ein kritischer abgesenkter Druck $p_{B s}$, ab dem der durch die Oberflächenspannung erzeugte Innendruck überwunden wird und eine Blase im Prinzip beliebig weit expandiert.

Bei der dynamischen Blake-Schwelle $p_{B d}$ wird der periodische (sinusförmige) akustische Anregungsdruck gesucht, ab dem die Oberflächenspannung überwunden wird und eine Blase deutlich über ihren Ruheradius aufschwingt. Die dynamische Blake-Schwelle liegt durch die zeitweisen Überdruck der Anregung etwas höher als die statische Blake-Schwelle.

\subsubsection{Statische Blake-Schwelle}

Die statische Blake-Schwelle wird von Young [39] wie im folgenden Abschnitt beschrieben.

Unter Vernachlässigung des Dampfdrucks sowie der Diffusion von Gas, kann der Blaseninnendruck $p_{i}$ im Gleichgewicht mit den auf die Blase wirkenden Kräften, die sich aus Umgebungsdruck und Oberflächenspannung zusammensetzen, sein. Die Blase hat den konstanten Radius $R_{n}$ und es gilt

$$
p_{i}=p_{\text {stat }}+\frac{2 \sigma_{O}}{R_{n}}
$$

Wird nun der Umgebungsdruck um einen statischen Druck der Größe $p_{A}$ reduziert, so wächst die Blase, bis sich ein neues Gleichgewicht einstellt. Im isothermen Fall gilt $p_{i} V_{n}=\bar{p}_{i} \bar{V}_{n}$ und somit folgt für das neue Gleichgewicht:

$$
\bar{p}_{i}=p_{i}\left(\frac{R_{n}}{\bar{R}_{n}}\right)^{3}=p_{\text {stat }}-p_{A}+\frac{2 \sigma_{O}}{\bar{R}_{n}}
$$

Diese Gleichung lässt sich nach der Druckminderung $p_{A}$ umstellen und stellt damit eine Funktion in Abhängigkeit des neuen Ruheradius $\bar{R}_{n}$ dar.

$$
p_{A}=p_{\text {stat }}+\frac{2 \sigma_{O}}{\bar{R}_{n}}-\left(p_{\text {stat }}+\frac{2 \sigma_{O}}{R_{n}}\right)\left(\frac{R_{n}}{\bar{R}_{n}}\right)^{3}
$$

Da diese Funktion nach oben beschränkt ist (siehe Abbildung 2.16), lässt sich ein maximaler Absenkungsdruck $p_{B s}$ finden, der bei dem sogenannten 
kritischen Radius $R_{\text {krit }}$ erreicht wird:

$$
\begin{aligned}
\frac{\partial p_{A}}{\partial \bar{R}_{n}} & =0 \\
& =\frac{-2 \sigma_{O}}{\bar{R}_{n}{ }^{2}}+3\left(p_{\text {stat }}+\frac{2 \sigma_{O}}{R_{n}}\right) \frac{R_{n}^{3}}{\bar{R}_{n}{ }^{4}} \\
\Rightarrow R_{\text {krit }} & =\sqrt{\left(\frac{3 R_{n}^{3}\left(p_{\text {stat }}+\frac{2 \sigma_{O}}{R_{n}}\right)}{2 \sigma_{O}}\right)}
\end{aligned}
$$

Bei einem Überschreiten des kritischen Radius $\bar{R}_{n}>R_{\text {krit }}$ sinkt die Oberflächenspannung zu stark ab, um den Innendruck der Blase auszugleichen und es gibt keine mögliche Gleichgewichtslage von $\bar{R}_{n}$ für $p_{A}$. Das hätte zur Folge, dass die Blase ungehindert expandiert, solange der Unterdruck $p_{A}$ besteht.

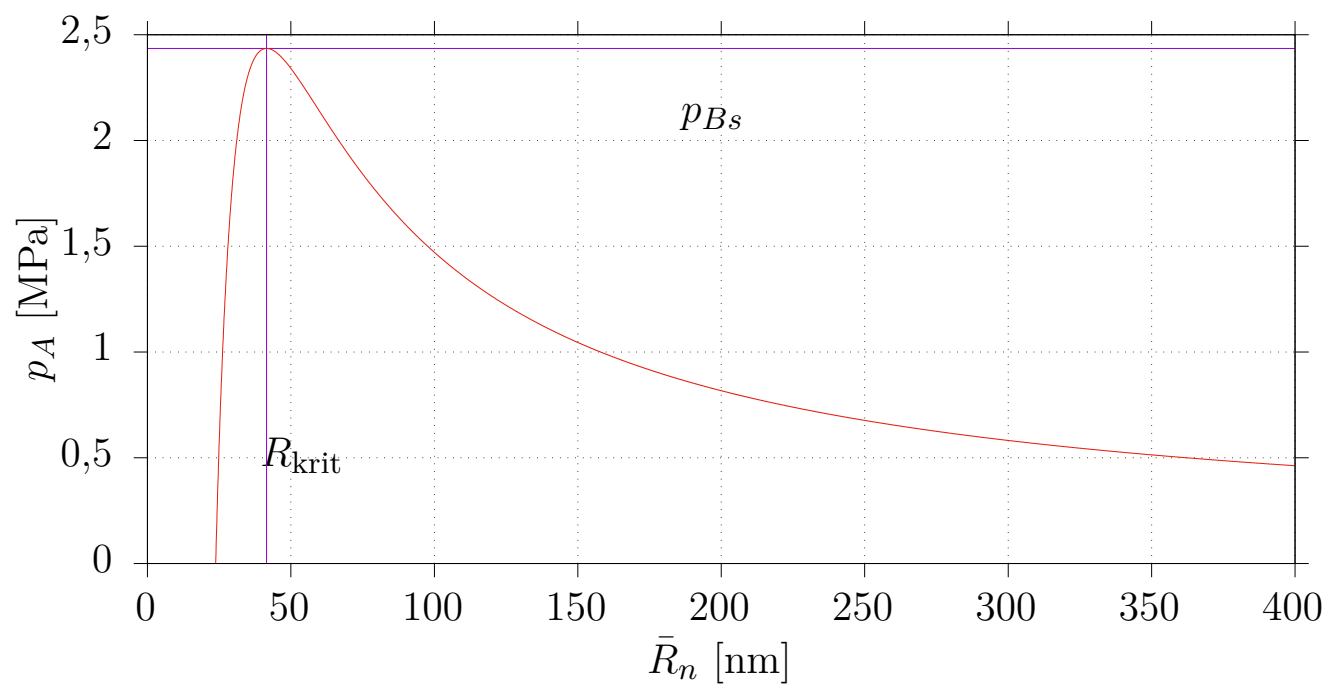

Abb. 2.16.: Die Kurve zeigt die Gleichung (2.8.4), in der $p_{A}$ in Abhängigkeit von $\bar{R}_{n}$ angegeben ist. Zusätzlich ist der kritische Radius $R_{\text {krit }}$ und die statische Blake-Schwelle $p_{B s}$ eingezeichnet. Für den Ruheradius, die Oberflächenspannung und den statischen Druck wurde $R_{n}=23,78 \mathrm{~nm}, \sigma_{O}=0,07275 \mathrm{~N} / \mathrm{m}$ und $p_{\text {stat }}=100 \mathrm{kPa}$ gewählt.

Wird der kritische Radius $R_{\text {krit }}$ in die Gleichung (2.8.4) eingesetzt, erhält man den von Blake [40] bestimmten Grenzwert des Unterdruckes, der eine Blase auf ihren kritischen Radius aufzieht.

$$
p_{B s}=p_{\text {stat }}+\frac{4 \sigma_{O}}{3} \sqrt{\frac{2 \sigma_{O}}{3 R_{n}^{3}\left(p_{\text {stat }}+\frac{2 \sigma_{O}}{R_{n}}\right)}}
$$

Da diese Betrachtungen mit statischen Annahmen gemacht wurden, spricht 
man bei $p_{B s}$ von der statischen Blake-Schwelle, die in Abbildung 2.17 für unterschiedliche Ruheradien dargestellt ist.

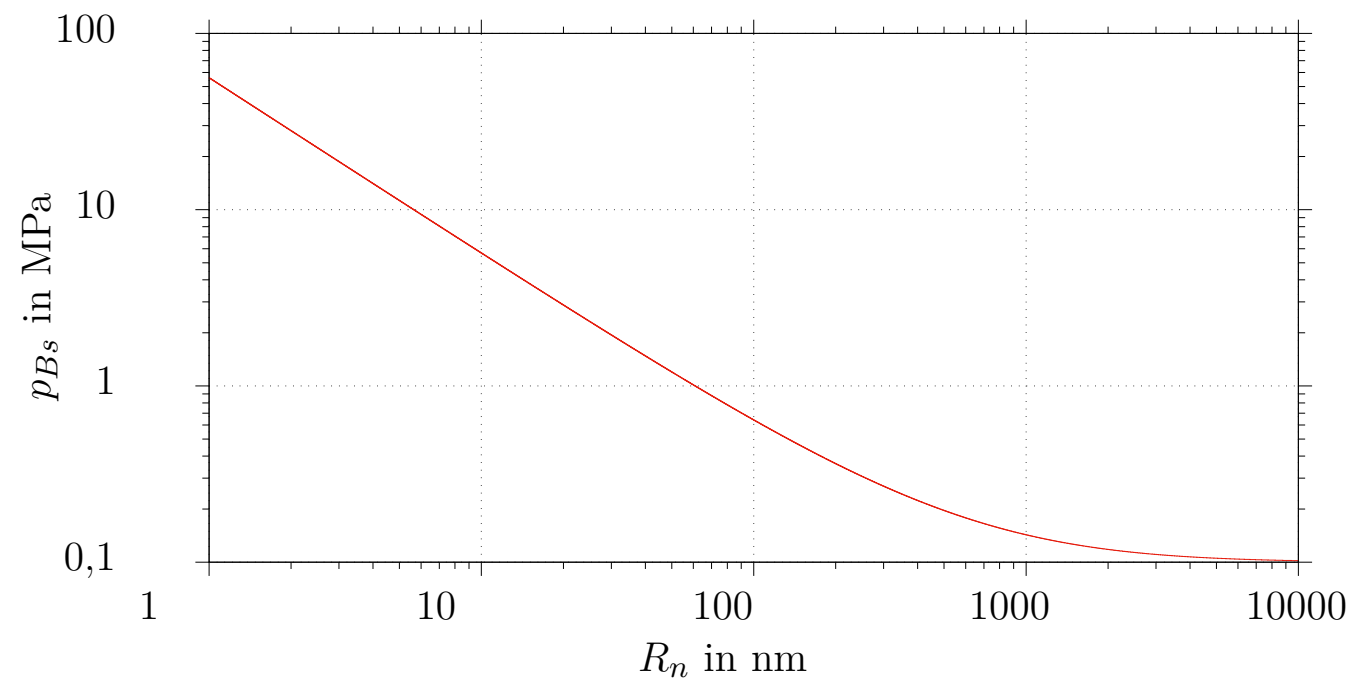

Abb. 2.17.: Zeigt den statischen Blake-Druck für unterschiedliche Ruheradien mit der Oberflächenspannung $\sigma_{O}=0,07275 \mathrm{~N} / \mathrm{m}$ und dem statischen Druck $p_{\text {stat }}=100 \mathrm{kPa}$.

\subsubsection{Dynamische Blake-Schwelle}

Während beim statischen Fall das Überschreiten der Blake-Schwelle zu einem unbegrenzten Wachstum führt, hat es bei einer periodisch getriebenen Blase eine starke nichtlineare Oszillation zur Folge, die einen eingeschwungenen Zustand erreichen kann. Der Übergang von schwacher zu starker Oszillation der Kavitationsblase wird als dynamische Blake-Schwelle bezeichnet.

In Abbildung 2.18 wird der maximale Radius einer Schwingung $R_{\max }$ oder $R_{\max } / R_{n}$ in Abhängigkeit vom Ruheradius $R_{n}$ für unterschiedliche akustische Anregungsdrücke dargestellt. 

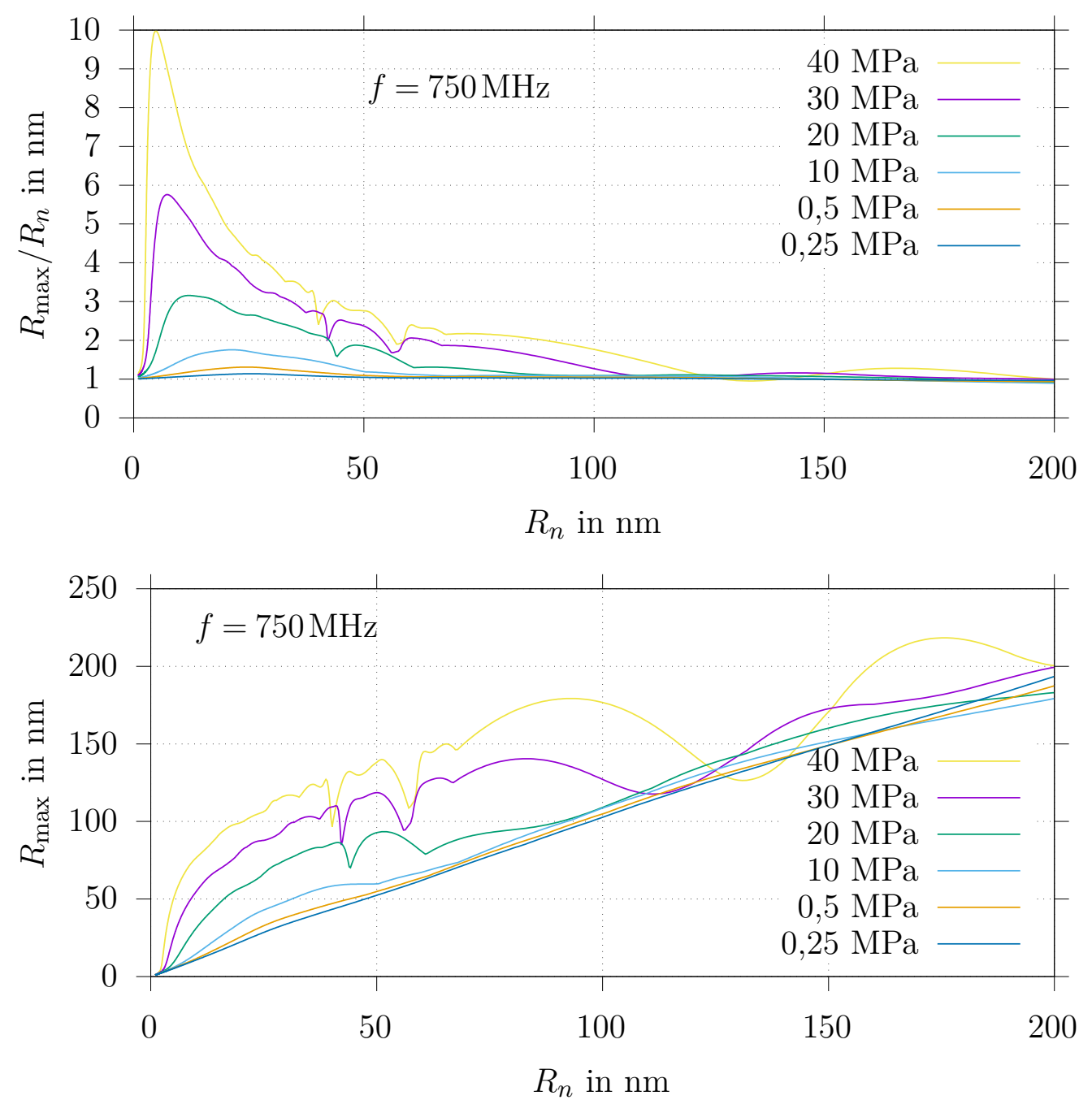

Abb. 2.18.: $R_{\max } / R_{n}$ gegen $R_{n}$ (oben) und $R_{\max }$ gegen $R_{n}$ (unten) für unterschiedliche Anregungsdrücke $p_{A}$ bei einer Anregungsfrequenz von $f=750 \mathrm{MHz}$.

Tab. 2.8.: Parameter für die dynamische Blake Schwelle

\begin{tabular}{|c|c|c|}
\hline$\sigma_{=}$ & $=0,07275 \mathrm{~N} / \mathrm{m}$ & Oberflächenspannung \\
\hline$\rho$ & $=998 \mathrm{~kg} / \mathrm{m}^{3}$ & Dichte \\
\hline$\mu$ & $=0,001 \mathrm{~Pa} \cdot \mathrm{s}$ & Viskosität \\
\hline$p_{v}$ & $=2330 \mathrm{~Pa}$ & Dampfdruck \\
\hline$p_{\text {stat }}$ & $=100 \mathrm{kPa}$ & Umgebungsdruck \\
\hline
\end{tabular}

Für die Berechnung der Abbildungen 2.18 2.21 wird das Keller-Miksis-Modell aus Abschnitt 2.8.1 verwendet mit den Parameter aus Tabelle 2.8. Die Anregungsfrequenz und der Anregungsdruck ist in den Abbildungen angegeben. 
Noch deutlicher ist der Übergang von geringer Blasenoszillation zu einer großen Dynamik bei der doppelt doppeltlogarhitmischen Darstellung in Abbildung 2.19 zu erkennen.

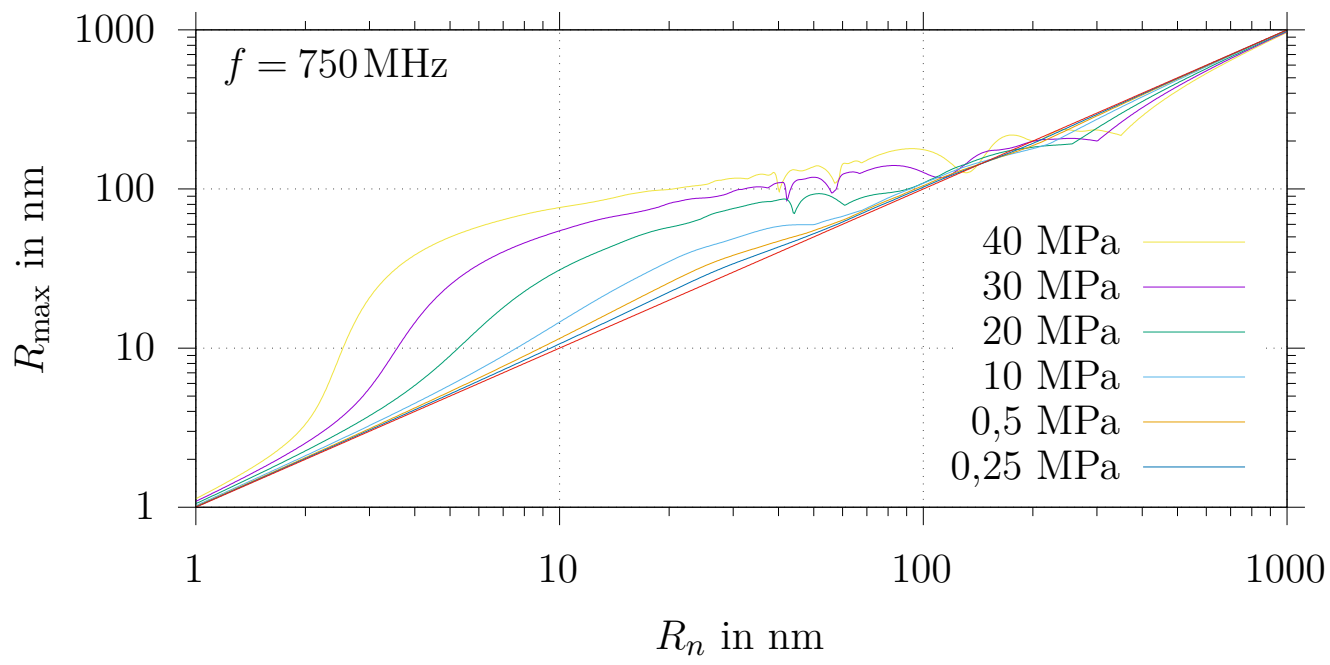

Abb. 2.19.: Doppeltlogarhitmische Auftragung von $R_{\max }$ gegen $R_{n}$ für unterschiedliche Anregungsdrücke $p_{A}$ bei einer Anregungsfrequenz von $f=750 \mathrm{MHz}$.

Bei geringeren Anregungsdrücken ist die Blasendynamik beim Resonanzradius am größten, verschiebt sich aber mit steigendem Druck zu geringeren Blasenradien. Mit einem Radius von $<100 \mathrm{~nm}$ sind die Blasen auch noch eine Größenordnung kleiner als eine Schallwelle mit $\lambda \approx 2 \mu \mathrm{m}$.

Im Vergleich zu niedrigeren Frequenzen wie in Abb. 2.20 dargestellt $(100 \mathrm{kHz}$ und $10 \mathrm{MHz}$ ) werden bei einer Anregung mit $750 \mathrm{MHz}$ deutlich höhere Drücke benötigt, um bei den sehr kleinen Blasen überhaupt eine Blasenschwingung zu erzeugen. 

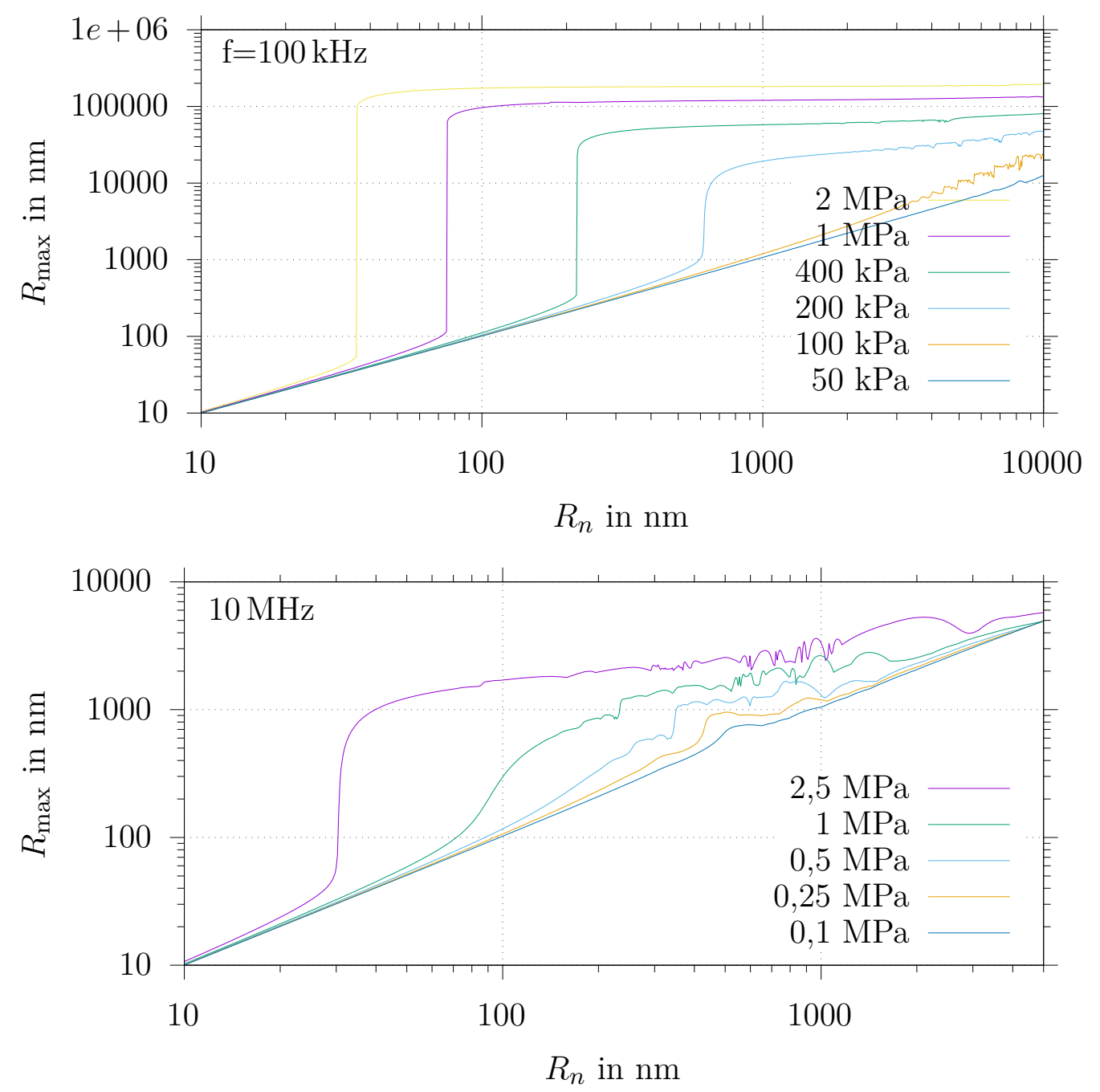

Abb. 2.20.: Doppeltlogarhitmische Auftragung von $R_{\max }$ gegen $R_{n}$ für eine Anregungsfrequenz von $f=100 \mathrm{kHz}$ (oben) und $f=10 \mathrm{MHz}$ (unten) bei unterschiedliche Anregungsdrücke $p_{A}$

Als Kriterium für das Überschreiten der dynamischen Blake-Schwelle wird das Erreichen eines maximalen Blasenradius $R_{\max }$ während einer Oszillation, der dem $N$-fachen des Ruheradius $R_{n}$ entspricht, gewählt. Typische Werte für $\mathrm{N}$ liegen zwischen zwei und zehn.

Betrachtet man die Parameterebenen in der Abbildung 2.21, die durch den Anregungsdruck $p_{A}$ und den Ruheradius $R_{n}$ aufgespannt werden, so sieht man, für welche Werte starke Blasenschwingungen zu erwarten sind. Größere Werte von $N$ bedeuten, dass Mehrfache des Ruheradius $R_{n}$ während einer Schwingung erreicht werden und dadurch ein heftigerer Kollaps zu erwarten ist. Diese unterschiedlichen Arten des Schwingungsverhaltens werden durch die dynamische Blake-Schwelle getrennt. 

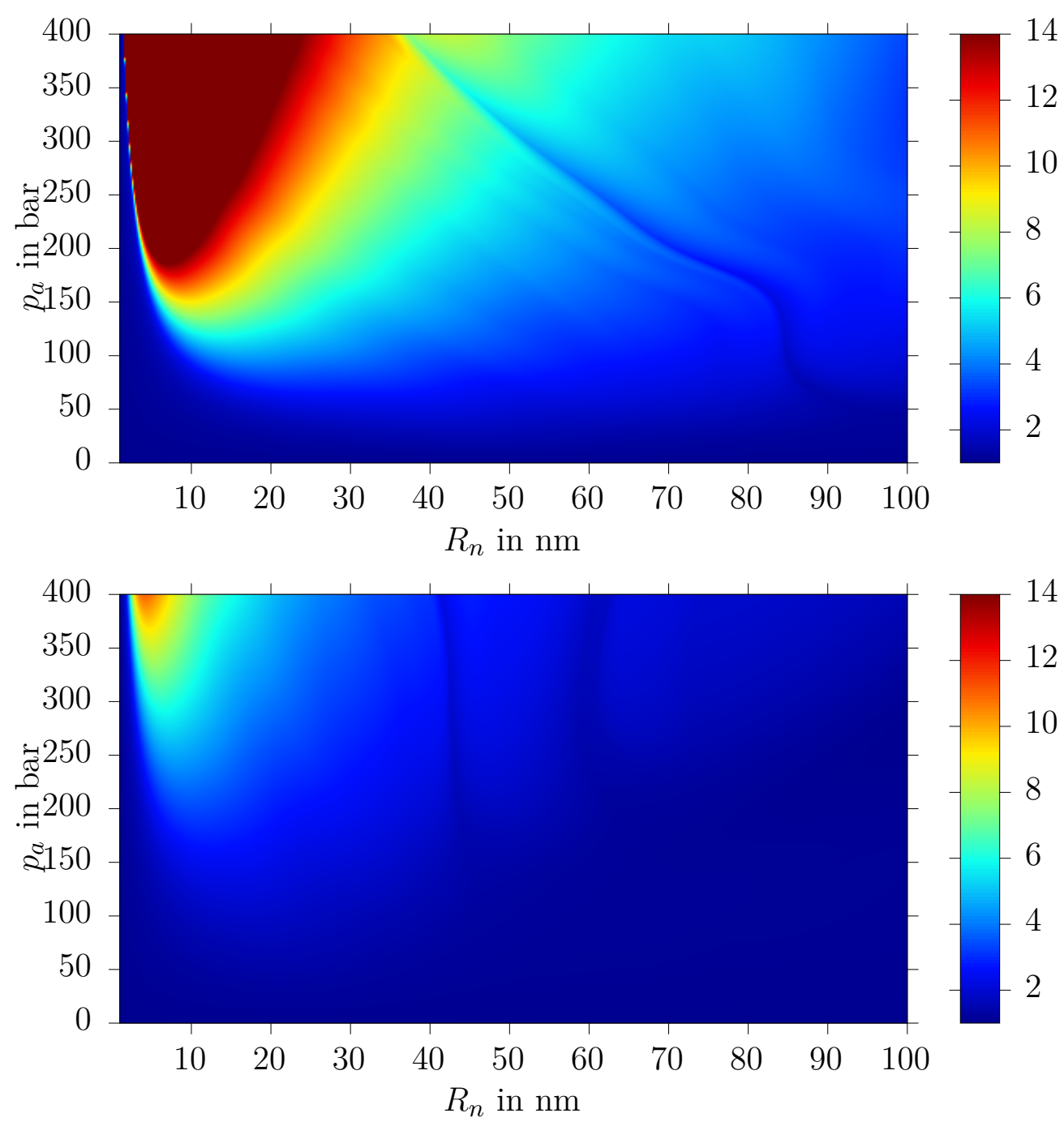

Abb. 2.21.: Darstellung der dynamischen Blake-Schwelle über den normierten Maximalradius $R_{\max } / R_{n}$ (farbig codiert) bei $f=250 \mathrm{MHz}$ (oben) und $f=750 \mathrm{MHz}$ (unten) in der Parameterebene für Druckamplitude $p_{A}$ und Ruheradius $R_{n}$.

Für eine Frequenz von $750 \mathrm{MHz}$ ist erst bei Drücken von über $\approx 100$ bar $(10 \mathrm{MPa})$ eine stärker oszillierende Blase zu erwarten. Voraussetzung für das Auftreten von Kavitation ist, dass in der Flüssigkeit Blasen mit einem Ruheradius von $1 \mathrm{~nm}$ bis $20 \mathrm{~nm}$ („Keimblasen“) vorhanden sind. 
2. Theorie 


\section{Simulationen}

Für die Simulationen in diesem Abschnitt wird die Simulationssoftware Comsol verwendet, die auf Finite-Elemente-Methode basiert. Zunächst wird die Schallausbreitung bei einem runden Schallwandler mit einem Durchmesser von $200 \mu \mathrm{m}$ simuliert, zum Einen in der Näherung der linearen Akustik und zum Anderen mit der nicht-linearen Akustik. Die beiden Resultate werden miteinander verglichen, und ein einfacheres Modell des Schallfeldes als eine ebene Welle wird eingeführt.

Im Weiteren wird für den linearen sowie nichtlinearen Fall die Volumenkraft berechnet, die durch das dissipierende Schallfeld erzeugt wird. Anschließend wird die Volumenkraft genutzt, um eine laminare Flüssigkeitsströmung zu simulieren, die im nächsten Abschnitt mit den experimentell gemessenen Strömungen verglichen werden können.

\subsection{Courant-Zahl}

Die Courant-Zahl (oder um alle Beteiligten zu nennen die Courant-FriedrichLewy-Zahl) wird in numerischen Strömungssimulationen als charakteristische Größe verwendet, um die Stabilität, Korrektheit und Genauigkeit zu beurteilen. Sie gibt an, um wie viel Rechenzellen sich eine betrachtete Größe pro Zeitschritt bewegt [41]:

$$
z_{c}=\frac{v \cdot \delta t}{\delta x}
$$

Diese Zahl ist für das explizite Euler-Verfahren entscheidend, da dieses nur für $z_{c}<1$ stabil ist: Die Strömung darf pro Zeitschritt nicht über eine Zelle hinwegströmen.

Auch für akustische Simulationen findet die Courant-Zahl Anwendung, nur wird hier nicht die Strömungsgeschwindigkeit $v$, sondern die Schallgeschwindigkeit $c$ berücksichtigt: Die Ausbreitung einer Schallwelle muss pro Zeitschritt möglichst auf eine Nachbarzelle beschränkt bleiben.

\subsection{Lineare Schallausbreitung}

Die lineare Schallausbreitung wird mit Gleichung (2.3.3) berechnet, um das Schallfeld eines Schallwandlers im Frequenzbereich von $150 \mathrm{MHz}$ bis $1500 \mathrm{MHz}$ 


\section{Simulationen}

zu simulieren. Dazu wurde ein 2d-rotations-symmetrischer Fall angenommen. Die z-Achse dient als Rotationsachse.

Der Radius des Wandlers beträgt $r_{w}=100 \mu \mathrm{m}$ und der Mittelpunkt liegt im Schnittpunkt der r-Achse und z-Achse. Für den Schallwandler wird die erste Oberflächenmode angenommen, also bei $r=0 \mu \mathrm{m}$ die maximale Auslenkung sowie die maximale Auslenkungsbeschleunigung $a$, die für die Simulation benötigt wird. Diese fällt bis $r=r_{w}$ dann kosinusförmig auf $0 \mathrm{ab}$, wie es von der Gleichung (3.2.1) beschrieben wird und in Abb. 3.1 dargestellt:

$$
a(r)= \begin{cases}a_{\max } \cdot \cos \left(0,5 \cdot \frac{\pi \cdot r}{r_{w}}\right) & \text { für } r \in\left[0, r_{w}\right] \\ 0 & \text { für } r \notin] 0, r_{w}[\end{cases}
$$

Das Rechengebiet umfasst $r=0 \mu \mathrm{m}$ bis $200 \mu \mathrm{m}$ und $z=0 \mu \mathrm{m}$ bis $500 \mu \mathrm{m}$ und wird mit Wasser gefüllt angenommen.

Alle Simulationsabbildungen mit der radialen Komponente $r$ werden an der Rotationsachse (z-Achse) gespiegelt dargestellt was bei der rotationssymmetrischen Rechnung einem Schnitt durch den Simulationszylinder entspricht. Daher geht z.B. die Abb. 3.1 von $-200 \mu \mathrm{m}$ bis $-200 \mu \mathrm{m}$.

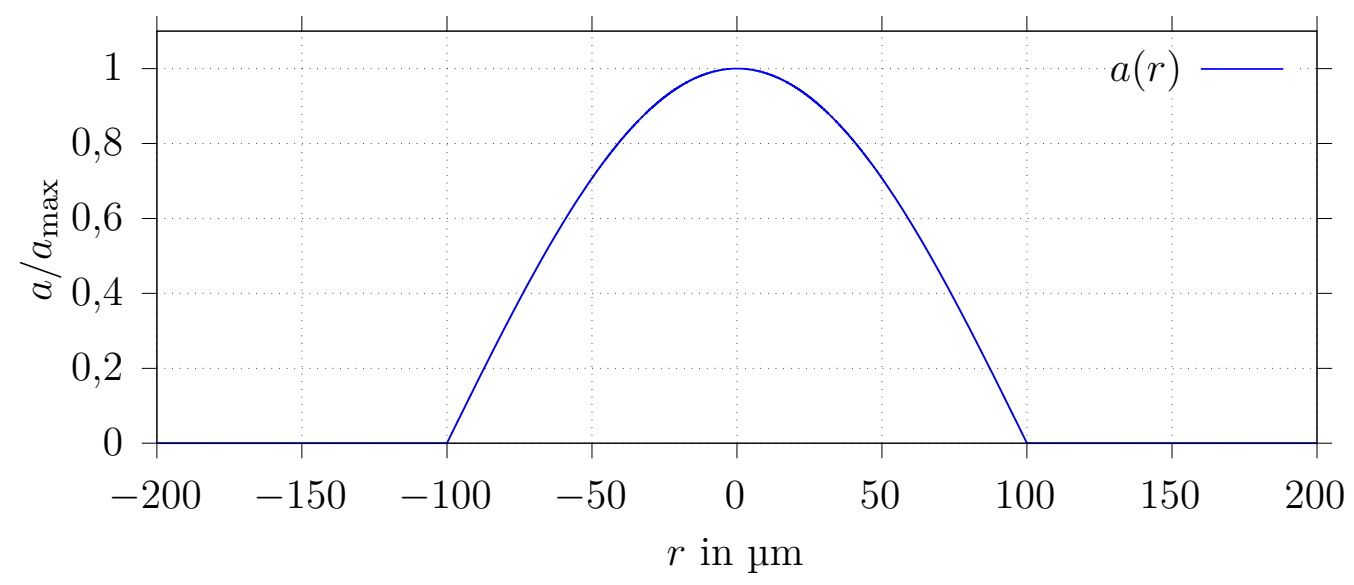

Abb. 3.1.: Wandlerbeschleunigung $a(r)$ nach Gleichung 3.2.1.

Im folgenden Teil wird exemplarisch eine Frequenz $f=750 \mathrm{MHz}$ für die Abbildungen verwendet.

Mit den Parametern aus Tabelle 3.1 ergeben sich für die numerische Simulation des Schallfeldes die in den Abbildungen 3.2 gezeigten Werte. 

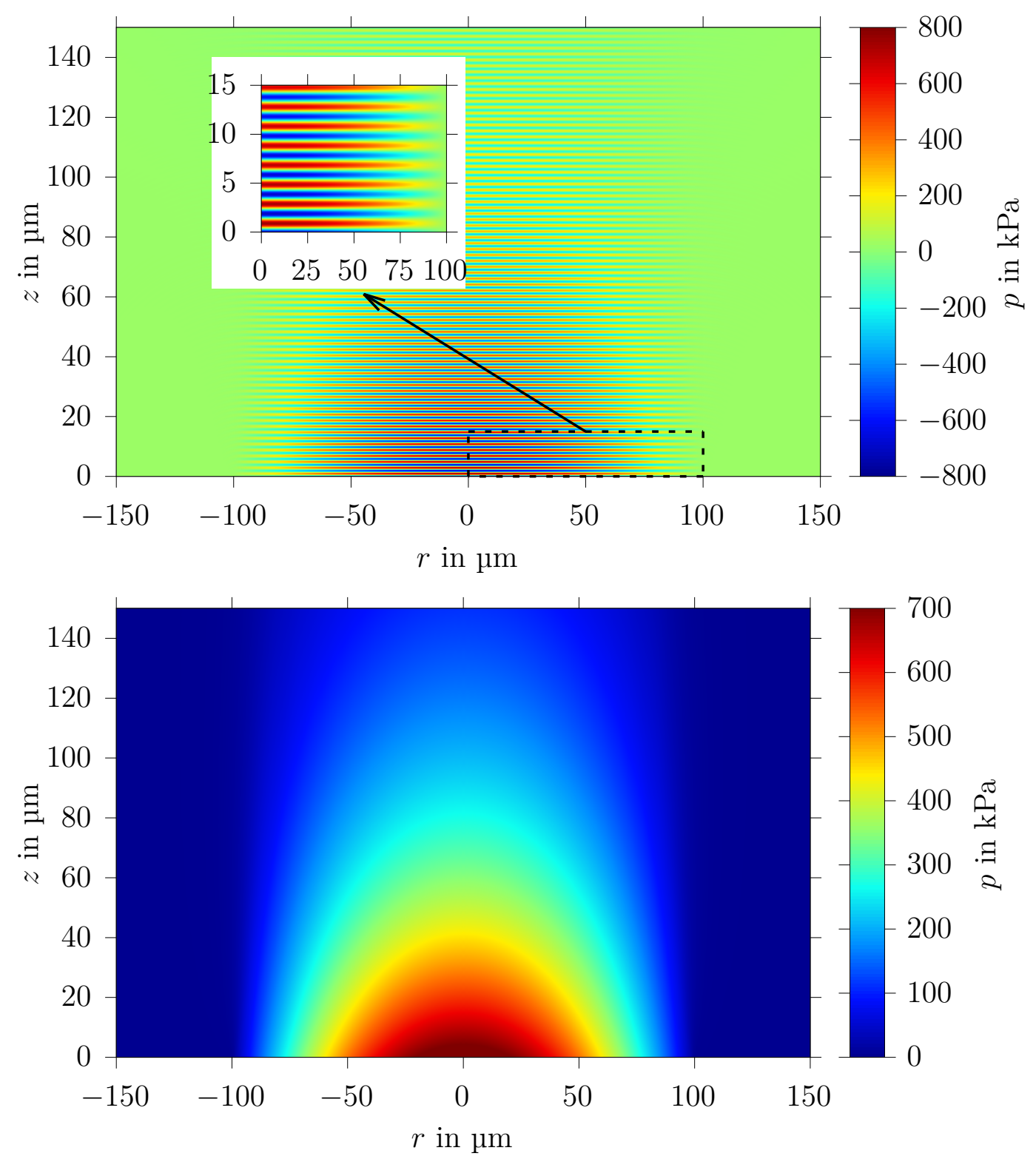

Abb. 3.2.: Schalldruckverteilung (oben) und Amplitude (unten) der in zRichtung laufenden Welle, berechnet mit linearer Akustik durch Comsol. Die Anregungsfrequenz beträgt $f=750 \mathrm{MHz}$; die weiteren Parameter können der Tabelle 3.1 entnommen werden.

Als Randbedingung wurde oben und rechts eine ebene Wellenabstrahlung angenommen. Die untere Grenze stellt der Wandler von $0 \mu \mathrm{m}$ bis $100 \mu \mathrm{m}$ dar, der durch eine beschleunigte Wand simuliert wird. Der Rest des unteren Rands wird als schallharte Grenze angenommen. Die $z$-Achse bei $r=0$ ist die Achse der Rotationssymmetrie. Für eine anschaulichere Abbildung wird der Simulationsbereich immer an der $z$-Achse gespiegelt dargestellt. Das Gitter der Simulation hat eine maximale Größe von $\lambda / 12$, um die Schallwellen auflösen 


\section{Simulationen}

zu können.

Für die Simulation wird als Fluid Wasser mit seinen Materialparametern bei $20^{\circ} \mathrm{C}$ angenommen. Für die akustische Anregung werden folgende Werte verwendet:

Tab. 3.1.: Parameter der numerischen Simulation

\begin{aligned} \hline \hline$r_{w} & =100 \mu \mathrm{m} & &$ Radius Schallwandler \\ $f= & 750 \mathrm{MHz} & &$ Frequenz \\ $\omega=2 \pi f= & 4712 \mathrm{MHz} & &$ Kreisfrequenz \\ $d_{\max } \approx & 0,11 \mathrm{~nm} & &$ Auslenkung \\ $v_{\max }=d_{\max } \cdot \omega & =0,5 \mathrm{~m} / \mathrm{s} & &$ Auslenkungsgeschwindigkeit \\ $a_{\max }=v_{\max } \cdot \omega & =2,36 \mathrm{Gm} / \mathrm{s}^{2} & &$ Auslenkungsbeschleunigung \end{aligned}

Bei der Berechnung wurden nur die viskosen Effekte, jedoch keine thermischen Effekte berücksichtigt. Da die Viskosität einen deutlich größeren Einfluss hat, wie im Kapitel zum Dämpfungskoeffizienten (2.4.1) erläutert, ist der daraus resultierende Fehler vernachlässigbar klein.

\subsection{Nichtlineare Schallausbreitung}

In diesem Abschnitt wird das Schallfeld mittels der Westervelt-Gleichung 2.6.2 aus Abschnitt 2.6.1 mit Comsol berechnet. Wie dort bereits gezeigt, ist für die Stärke der Nichtlinearität der Schalldruck ausschlaggebend.

Die Geometrie sowie die Randbedingungen werden wie bei der linearen Berechnung aus Abschnitt 3.2 gewählt. Wegen des steigenden Rechenaufwandes durch das benötigte feinere Simulationsgitter wird in z-Richtung nur bis $60 \mu \mathrm{m}$ gerechnet.

Abbildung 3.3 (oben) zeigt die Amplitude eines nichtlinear gerechneten Schallfeldes mit einer relativ geringen Wandlerauslenkung von 0,11 nm im Zentrum des Wandlers. Dies entspricht einer Partikelgeschwindigkeit von $u_{0}=0,5 \mathrm{~m} / \mathrm{s}$ bzw. einem Druck von $p_{\max } \approx 740 \mathrm{kPa}$. 

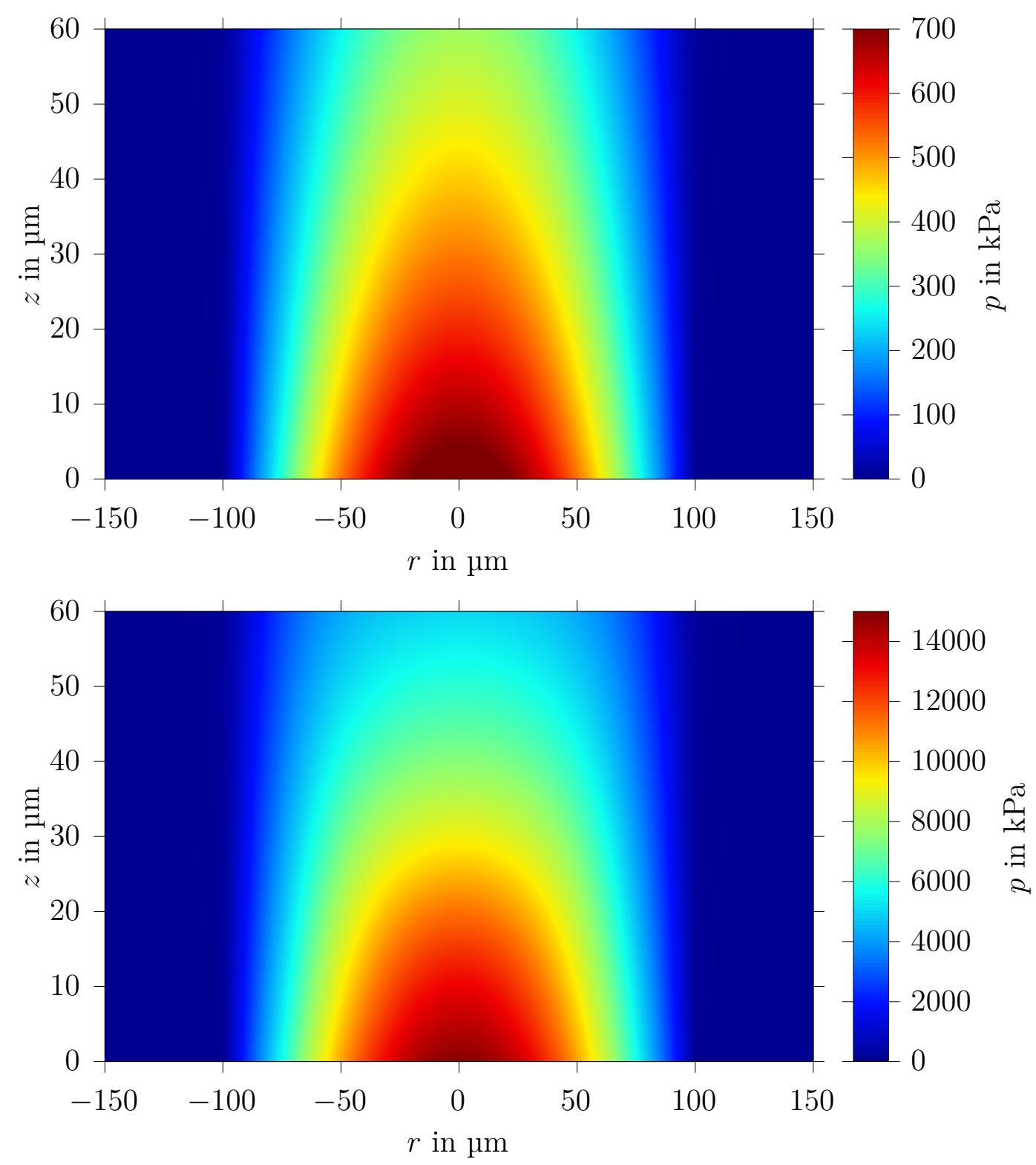

Abb. 3.3.: Amplitude mit nichtlinear gerechnetem Schallfeld bei einer Wandlerauslenkungsgeschwindigkeit von $u_{\max }=0,5 \mathrm{~m} / \mathrm{s}$ (oben) und $u_{\max }=10 \mathrm{~m} / \mathrm{s}$ (unten).

Bei der kleinen Auslenkung ist das Schallfeld nahezu identisch mit der linearen Lösung in Abb. 3.2 .

Bei der größeren Auslenkung, wie in Abbildung 3.3 unten dargestellt ist, zeigt sich das nichtlineare Verhalten. Hierzu wird bei der Simulation eine Auslenkung von $d_{\max }=2,12 \mathrm{~nm}$ im Zentrum des Wandlers angenommen, was einer maximalen Auslenkungsgeschwindigkeit von $u_{\max }=10 \mathrm{~m} / \mathrm{s}$ bzw. einem Schalldruck am Wandler von $p_{\max } \approx 15 \mathrm{MPa}$ entspricht.

Der Unterschied zwischen der niedrigen und der hohen Auslenkung in der Ab- 


\section{Simulationen}

bildung 3.3 ist neben der Maßeinteilung der Farbskala, dass die Amplitude in z-Richtung deutlich schneller absinkt. Dies ist durch die Nichtlinearität, wodurch höheren Frequenzen im Schallfeld angeregt werden, die wiederum stärker dissipieren, auch zu erwarten. Neben der Amplitude wird in Abbildung 3.4 auch die Schwingungsform der beiden nichtlinearen Lösungen verglichen.

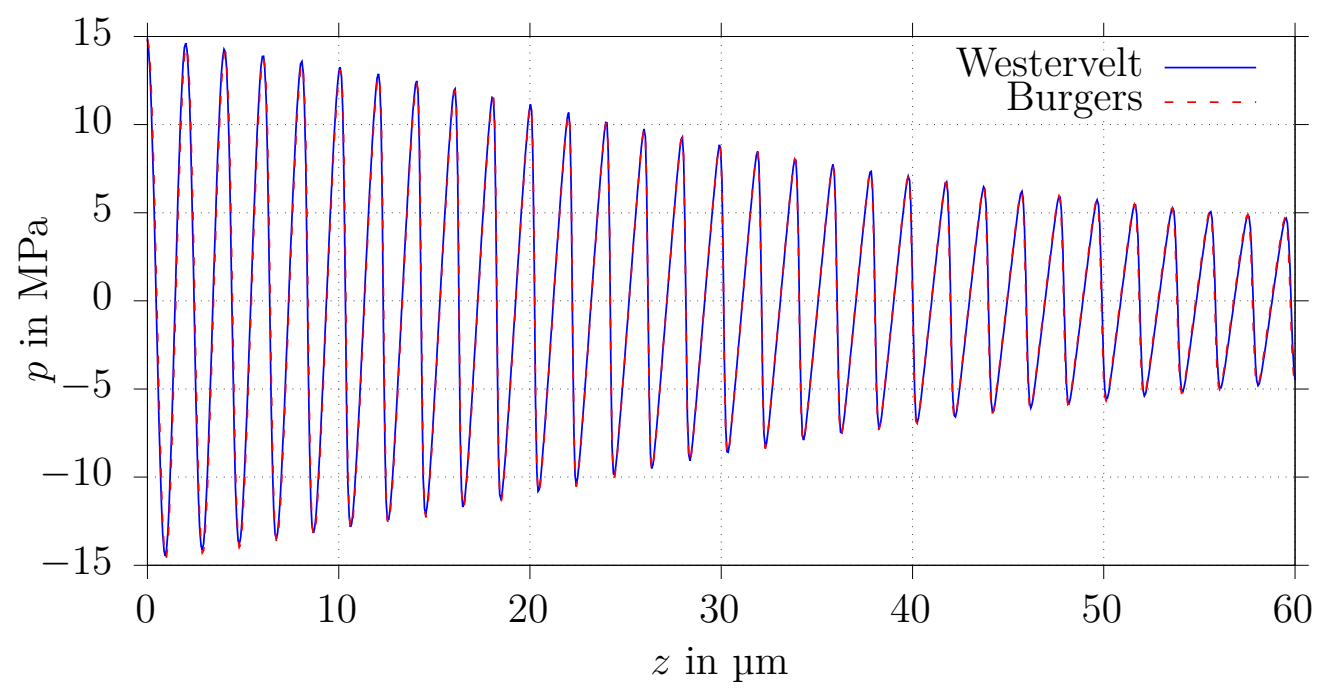

Abb. 3.4.: Vergleich von $p(z)$ bei $\mathrm{r}=0 \mu \mathrm{m}$ und festem $t$ der numerischen Lösung der Westervelt-Gleichung (Comsol) mit der Burgers-Gleichung.

Abbildung 3.5 (oben) vergleicht die Amplitude auf der Rotationsachse $(r=0)$ für die Westervelt-Gl. von Abbildung 3.3 (unten) mit der Amplitude, die durch eine einfache Dämpfung aus Abschnitt 2.4.1 zu erwarten wäre, und der exakten nichtlinearen Lösung der Burges-Gleichung für eine ebene Welle aus Abschnitt 2.6.7.

Da das hier simulierte Schallfeld keine einfache ebene Welle darstellt, sondern mit größer werdendem $r$ sich die Auslenkung des Wandlers und damit die Amplitude des Schallfeldes verringert, wird neben der Symmetrieachse auch bei $r=55$ und 94um das Schallfeld der Simulation mit dem einer linearen ebenen Welle einer geringeren Amplitude verglichen. 

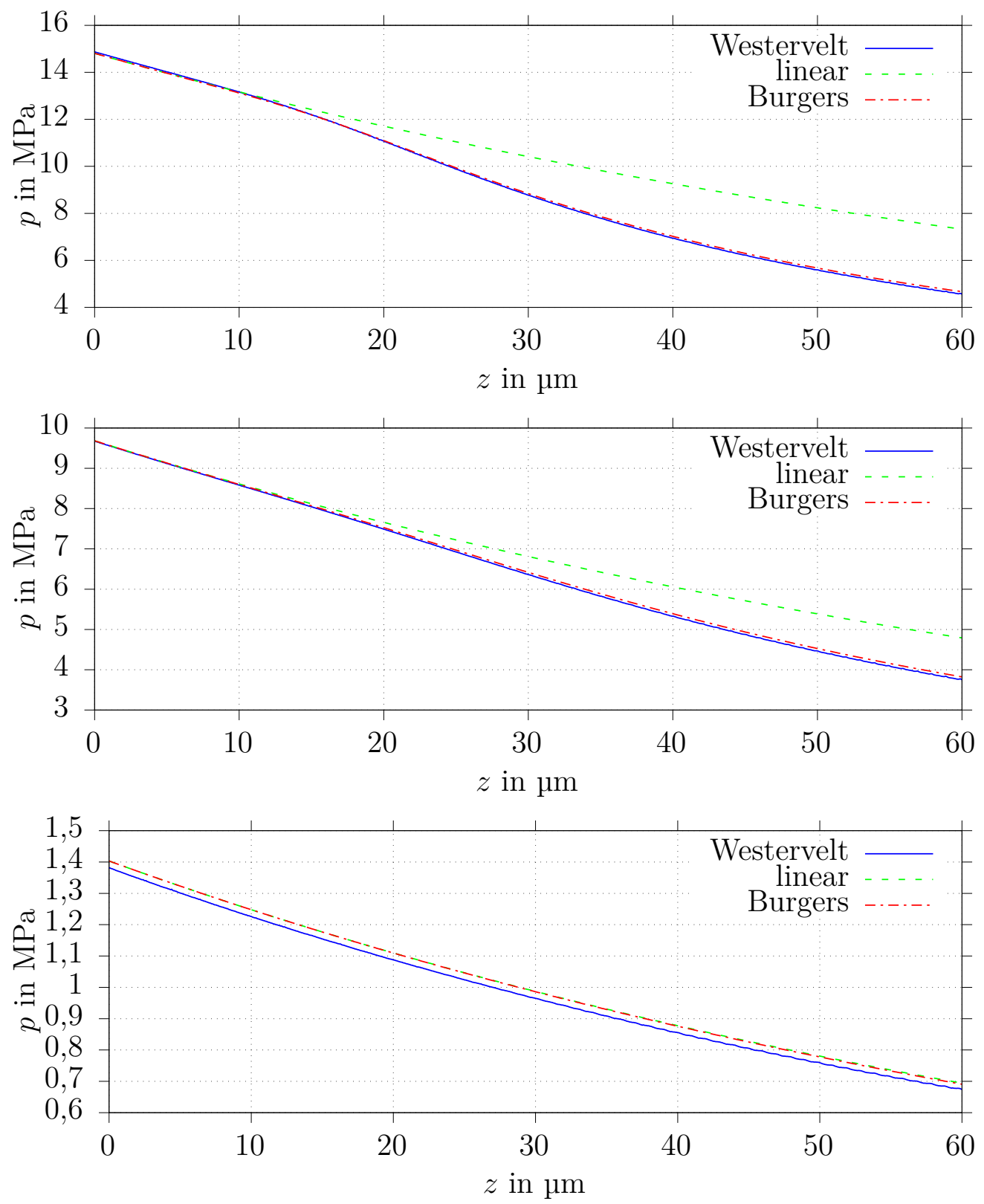

Abb. 3.5.: Vergeleich der Amplitude des Schallfeldes bei $r=0 \mu \mathrm{m}$ (oben), $r=55 \mu \mathrm{m}$ (mitte) und $r=94 \mu \mathrm{m}$ (unten) der numerischen Comsol Rechnung der Westerveltgleichung im Vergleich zu einer linearen dissipierenden Schallwelle und der nichtlinearen Burgers-Gleichung.

Wie in der Abbildung 3.5 gut zu erkennen ist, beeinflusst die Nichtlinearität insbesondere die Bereiche über dem Zentrum des Schallwandlers, der mit einer maximalen Auslenkungsgeschwindigkeit von $v_{\max }=10 \mathrm{~m} / \mathrm{s}$ simuliert wurde. Bei einer Partikelgeschwindigkeit von $u_{0}=10 \mathrm{~m} / \mathrm{s}$, was bei $750 \mathrm{MHz}$ in Wasser einem $R e_{\mathrm{ak}} \approx 6,4$ entspricht, ist ein nichtlineares Verhalten auch zu erwarten. Die Amplitude der linear dissipierenden Welle weicht im Zentrum 


\section{Simulationen}

deutlich von den Lösungen, die die Nichtlinearität berücksichtigen, ab, aber der Unterschied wird zum Rand des Schallwandlers hin mit sinkender Amplitude kleiner.

Die 1d numerische Lösung des nichtlinearen Schallfeldes durch die BurgersGleichung liefert nahezu das gleiche Ergebnis wie die 2d-rotations-symmetrische numerische Lösung mit der Westervelt-Gleichung. Die hohe Anforderung ans Gitter der 2d-rotations-symmetrischen numerischen Rechnung und die damit verbundene Dauer der Berechnung gibt Anlass, das Schallfeld durch eine ebene Welle zu nähern, wie im nächsten Abschnitt 3.4 gezeigt wird.

\subsection{Einfache Näherung des Schallfelds in analytischer Form, ebene dissipierende Welle}

\subsubsection{Ebene dissipierende Welle in linearer Näherung}

In diesem Abschnitt wird versucht, das Schallfeld in analytischer Weise als eine ebene exponentiell abklingende fortschreitende Welle darzustellen. Dazu wird der Faktor $e^{-\alpha z}$ als Dämpfung in z-Richtung verwendet. In seitliche Richtung (r-Richtung) wird eine kosinusförmig abnehmende Amplitude angenommen. Dazu kommt noch die sinusförmige Modulation einer laufenden Welle in Raum und Zeit, um den Wellencharakter zu berücksichtigen.

Mit diesen Annahmen ergibt sich die Gleichung (3.4.1), mit der sich ein Schallfeld berechnen lässt, das dem numerisch berechneten ähneln soll. Für den Schalldruck ergibt sich

$$
p(r, z, t)= \begin{cases}p_{0} \cdot \cos \left(0,5 \cdot \pi \cdot r / r_{w}\right) \cdot e^{-\alpha z} \cdot \sin (2 \pi f t-k z) & \text { für } r \in\left[0, r_{w}\right] \\ 0 & \text { für } r \notin] 0, r_{w}[.\end{cases}
$$

Die Schalldruckamplitude aus Gleichung (3.4.1) wird in der Abbildung 3.6 dargestellt. Soweit es für diese einfache Näherung nötig ist, wurden die Parameter wie in Abschnitt 3.2 gewählt. Als Anregungsdruck $p_{0}$ wird der maximale Druck der numerischen Rechnung aus Abschnitt 3.2 verwendet, um die Schallfelder vergleichen zu können. 

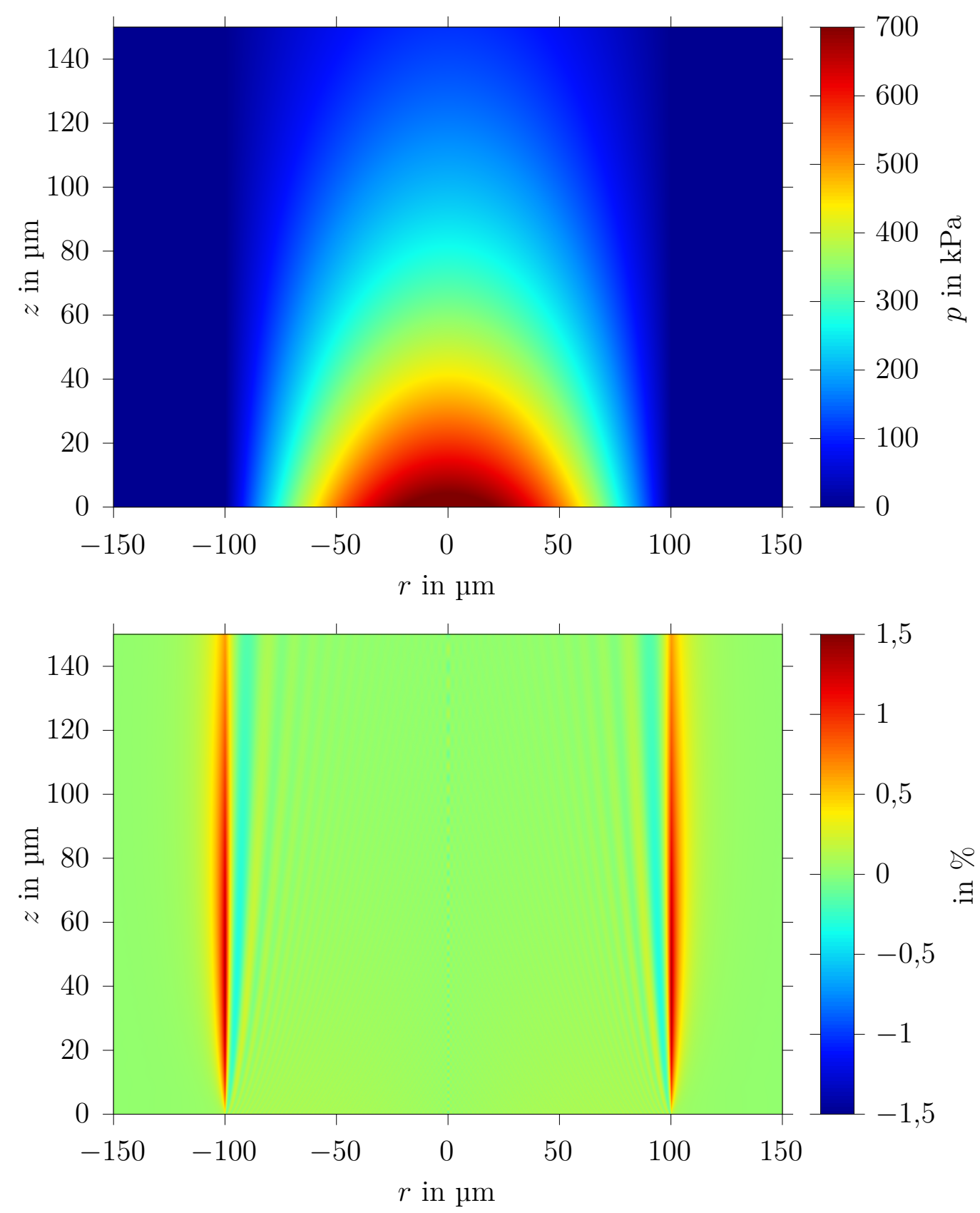

Abb. 3.6.: Amplitude des Schallfeldes von der Näherung als ebene Welle (oben) und Differenz zur lineare Lösung der Schallausbreitung aus Abschnitt 3.2 (unten).

Auf den ersten Blick gibt es kaum Unterschiede zwischen der linearen akustischen Lösung der Helmholtz-Gleichung aus Abschnitt 3.2 und der hier gezeigten, einfachen analytischen Näherung. Die Unterschiede werden erst durch die Differenz der beiden Schallfeldamplituden sichtbar wie in der Abbildung 3.6 (unten) gezeigt. 


\section{Simulationen}

Die größten Differenzen sind vor dem Wandler sowie an den seitlichen Rändern des Wandlers zu erkennen, an denen es in der numerischen Lösung „Keulen“ gibt. Diese Beugungseffekte sind im vereinfachten Modell vernachlässigt. Dies erscheint aber in der Regel akzeptabel, denn verglichen mit der maximalen Amplitude sind die Differenzen um circa drei Größenordnungen kleiner. Da der Abstrahlwinkel sehr klein ist, ist auch die seitliche Beugung während der Ausbreitung sehr klein.

Wie bereits gezeigt, lässt sich mit Gleichung 2.7.17) die Volumenkraft einer ebenen Welle berechnen. Sie wird in Abbildung 3.7 für das mit Gleichung (3.4.1) genäherte Schallfeld berechnet.

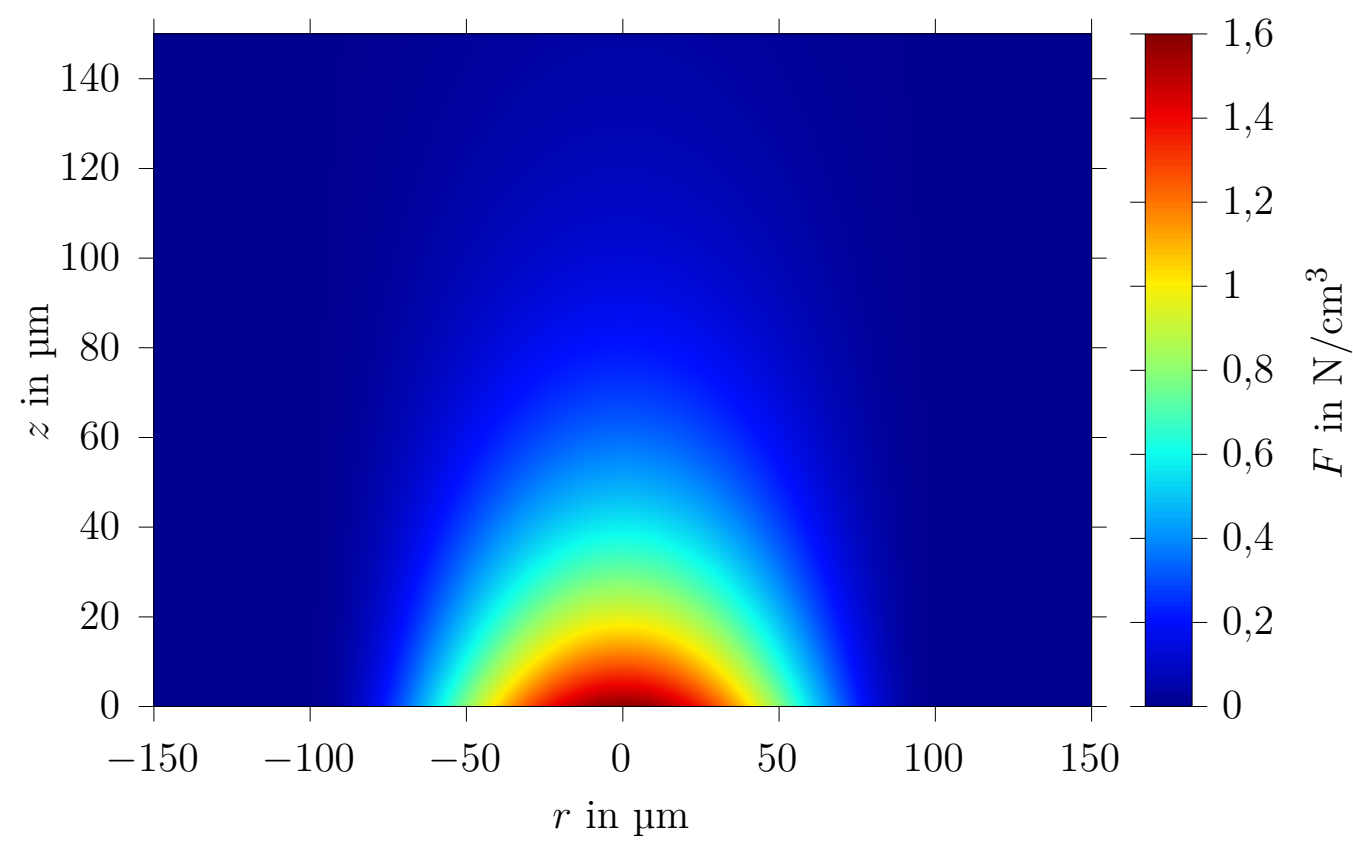

Abb. 3.7.: z-Komponente der Volumenkraft für eine Auslenkung von $d_{\max }=$ $0,11 \mathrm{~nm}$, was einer Partikelgeschwindigkeit von $u_{0}=0,5 \mathrm{~m} / \mathrm{s}$ entspricht bzw. einem Druck von $p_{\max } \approx 740 \mathrm{kPa}$.

Wie bereits in Abschnitt 2.7 .3 gezeigt, fällt die Volumenkraft mit $e^{-2 \alpha z} \mathrm{ab}$, im Gegensatz zur Amplitude des Schallfeldes, die nur mit $e^{-\alpha z}$ sinkt.

\subsubsection{Ebene dissipierende Welle in nichtlinearer Näherung}

Die oben gezeigte Näherung ist für geringe Amplituden gut geeignet, aber sobald man sich einer $R e_{\mathrm{ak}} \approx 1$ nähert, siehe Abschnitt 2.5.2, sollten die Nichtlinearitäten nicht vernachlässigt werden. Im Folgenden wird wie bei der linearen Näherung aus Abschnitt 3.4.1 die Schallwelle als ebene Welle mit einer seitlich 
abklingenden von $r$ abhängigen Abstrahlamplitude angenähert:

$$
p_{0}(r)=p_{0}(r=0) \cos \left(\frac{0,5 \cdot \pi \cdot r}{r_{w}}\right)
$$

für $|r|<r_{\mathrm{w}}$, sonst 0 .

In $z$-Richtung wird die Ausbreitung der Welle mit der Burgers-Gleichung aus Abschnitt 2.6.7 berechnet. Abbildung 3.8 zeigt die Amplitude eines Schallfeldes, das mit der oben beschriebenen Methode berechnet wurde.

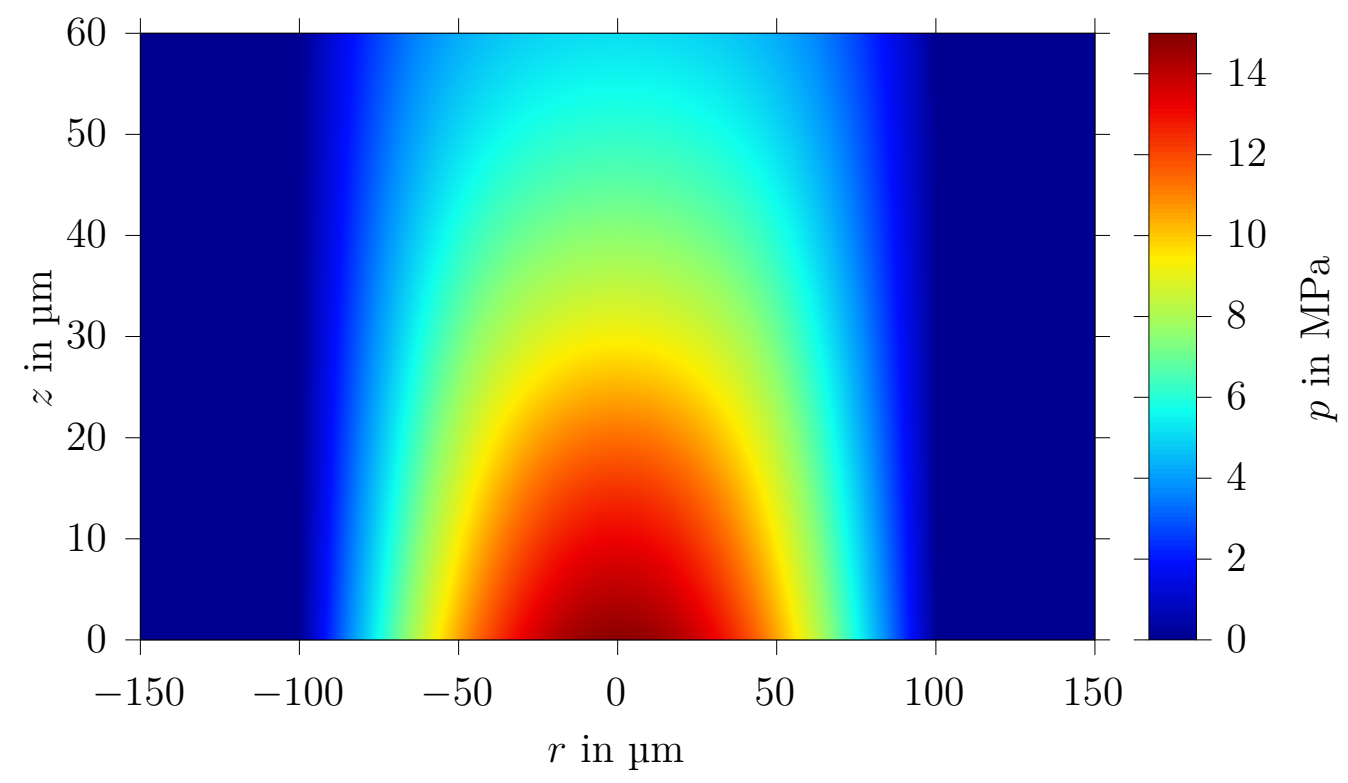

Abb. 3.8.: Schalldruckamplitude der nichtlinearen Näherung mit $u_{0}(r=0)=$ $10 \mathrm{~m} / \mathrm{s}$.

Durch Bilden der Differenz des numerisch berechneten Schallfeldes mittels der Westervelt-Gleichung (Abb. 3.3 (unten)) und der Näherung als nichtlineare ebene Welle (Abb.3.8) ergibt sich die Abbildung 3.9.

Die Differenzen sind mit einem Prozent der maximalen Amplitude relativ klein. Da die Näherung für die Berechnung der Volumenkraft benötigt wird, sind gerade die Bereiche in der Nähe des Schallwandlers entscheidend, die durch die Näherung recht gut wiedergegeben werden. 


\section{Simulationen}

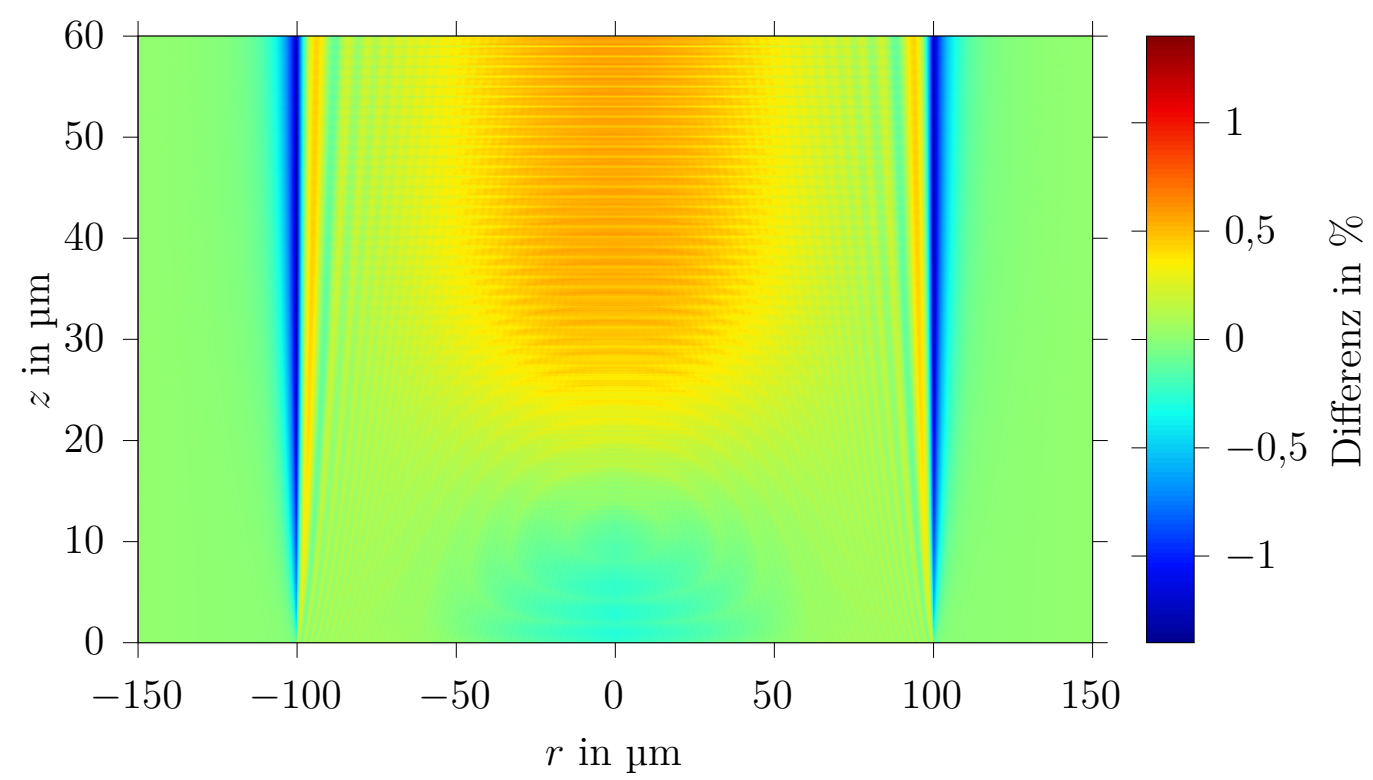

Abb. 3.9.: Differenz von 3.8 zur numerische gelösten Westerveltgleichung aus Abschnitt 3.3 .

Für Abbildung 3.10 wird das Schallfeld der nichtlinearen Näherung verwendet, um mit Gleichung (2.7.11) die resultierende Volumenkraft zu berechnen.

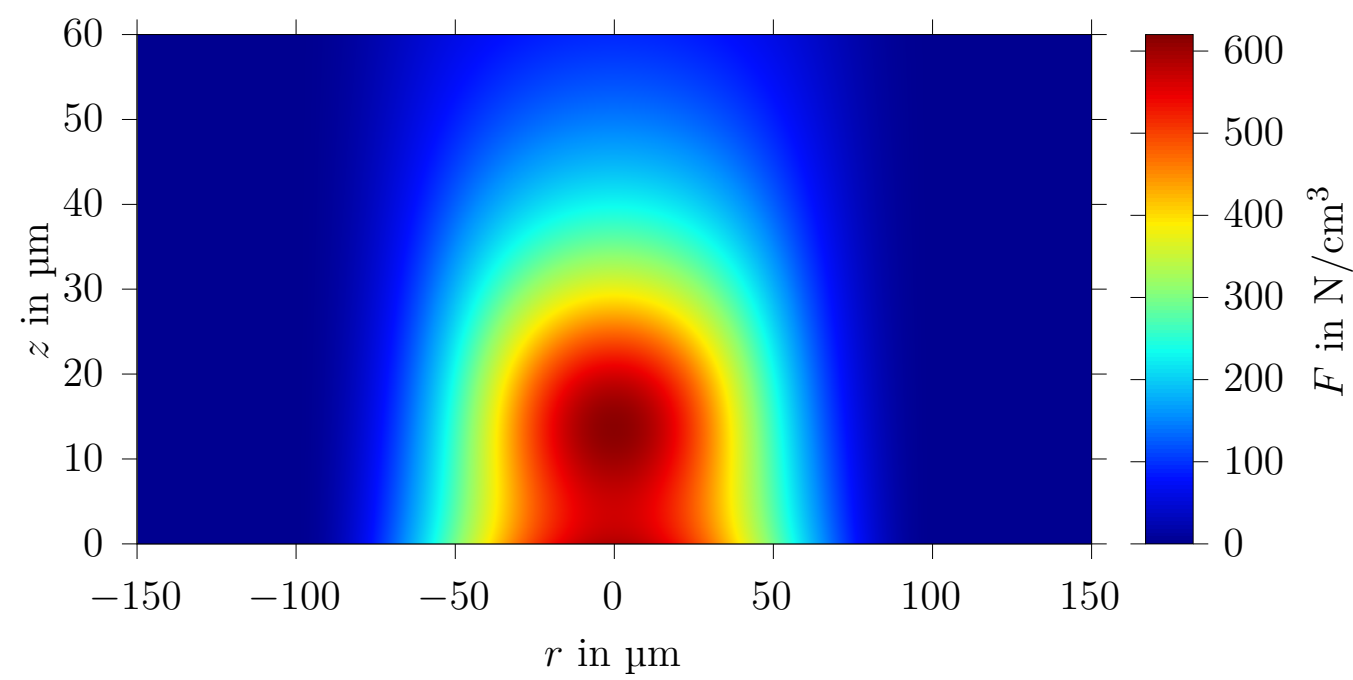

Abb. 3.10.: Volumenkraft in z-Richtung für die nichtlineare Näherung einer ebenen Welle mit $u_{0}=10 \mathrm{~m} / \mathrm{s}$.

Während im linearen Fall die maximale Amplitude direkt am Zentrum des Wandlers liegt, verschiebt sich durch die Nichtlinearität das Maximum in die Flüssigkeit hinein, wie in Abschnitt 2.7.4 „Volumenkraft einer dissipierenden ebenen Welle nichtlinear" erwartet wird. 
Durch die akzeptable Näherung des Schallfeldes kann die resultierende Volumenkraft einfacher berechnet werden. Die so erhaltene Volumenkraft kann dann für Strömungssimulationen verwendet werden, die eine gröbere Auflösung haben können, da nicht die Schallwellen aufgelöst werden müssen. Die Bedingung für die getrennte Berechnung von Schallfeld und Strömung wurde bereits in Abschnitt 2.2.2 diskutiert.

\subsection{Strömung durch Volumenkraft}

\subsubsection{Strömung durch Volumenkraft eines linear dissipierende Schallfeldes}

Mit der einfachen linearen Näherung des Schallfelds und der daraus resultierenden Volumenkraft aus Abschnitt 3.4.1 wird im Folgenden die resultierende Strömung berechnet.

Die durch die Volumenkraft entstehende Strömung wird mit Comsol in einer 2d-rotations-Symmetrie als eine laminare Strömung simuliert, dabei wird die obere und rechte Grenze als offen angenommen, der untere Rand wird als feste 'no slip' Grenze simuliert, und die $z$-Achse bei $r=0$ ist die Achse der Rotationssymmetrie. Als Startbedingung wird das Fluid in Ruhe angenommen. Die Abbildungen werden wie beim Schallfeld an der $z$-Achse gespiegelt dargestellt.

Die durch die Volumenkraft entstehende stationäre Strömung die aus einem Schallfeld mit $u_{0}=0,5 \mathrm{~m} / \mathrm{s}$ nach der Näherung aus Abschnitt 3.2 resultiert, ist in Abb. 3.11 gezeigt.

Bei der Strömung sind mehrere Effekte zu bemerken. Die durch die Volumenkraft entstehende Strömung ist zeitabhängig; es braucht eine gewisse Zeit, bis sich eine stationäre Strömung ausbildet. Außerdem reicht die Strömung deutlich weiter als die Volumenkraft bzw. wie das Schallfeld. Das Fluid wird oberhalb des Schallwandlers beschleunigt und strömt in die Ausbreitungsrichtung des Schallfeldes. Dabei erreicht die Strömung in einem Abstand von circa $85 \mu \mathrm{m}$ über dem Wandler eine maximale Geschwindigkeit von $13 \mathrm{~cm} / \mathrm{s}$. 


\section{Simulationen}

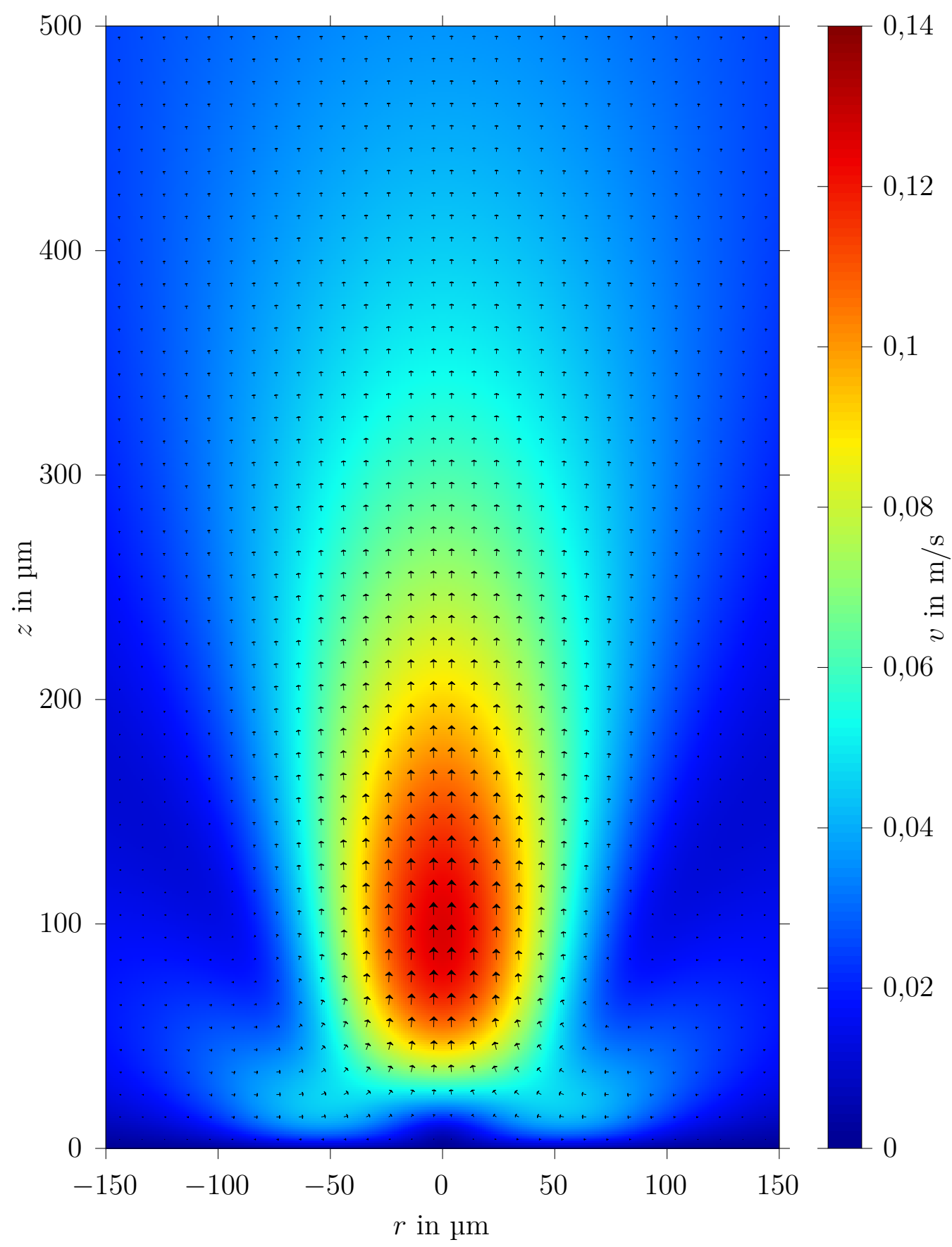

Abb. 3.11.: Der Betrag der Strömungsgeschwindigkeit ist durch die Farbskala wiedergegeben. Zusätzlich zeigen die Pfeile die Strömungsrichtung an. Als treibende Kraft wird die Volumenkraft ähnlich zur Abbildung 3.7 verwendet, resultierend aus einem Schallfeld mit $u_{0}=0,5 \mathrm{~m} / \mathrm{s}$.

In Abbildung 3.12 ist die Strömungsgeschwindigkeit auf der Rotationsachse, also auf der $z$-Achse, für unterschiedliche Zeiten dargestellt. Es dauert etwa 
$200 \mathrm{~ms}$ bis sich im 1,5 mm langen Simulationsgebiet eine stationäre Strömung ausbildet. Die Volumenkraft, wie in Abb. 3.7 gezeigt, beschleunigt die Flüssigkeit nur relativ nah am Schallwandler und reicht nur etwa $150 \mu \mathrm{m}$ in das Fluid. Die weitere Strömung wird nur durch Impulserhaltung aufrecht erhalten.

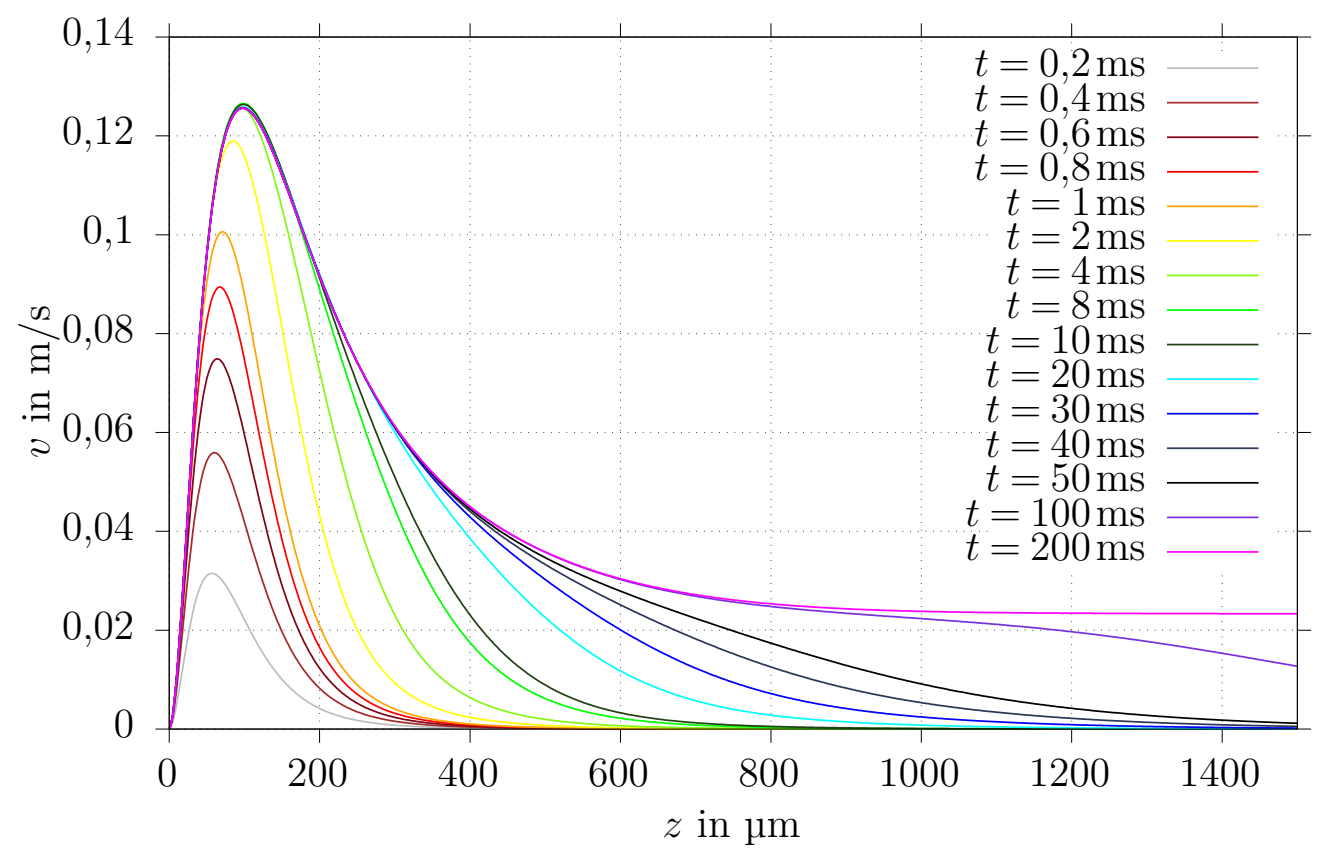

Abb. 3.12.: Strömungsgeschwindigkeit auf der z-Achse für unterschiedliche Zeiten nach dem Anschalten. Die Transducer-WandGeschwindigkeit, die zur Berechnung der Volumenkraft durch ein linear dissipierendes Schallfeld genutzt wurde, ist $u_{0}=0,5 \mathrm{~m} / \mathrm{s}$ bei einer Frequenz von $f=750 \mathrm{MHz}$

Wenn man die oben gemachten Berechnungen für unterschiedliche Frequenzen durchführt und die Strömungsgeschwindigkeiten auf der Rotationsachse im stationären Zustand vergleicht, ergibt sich Abbildung 3.13 .

Je höher die Frequenz ist, umso stärker wird die Schallwelle dissipiert und dadurch die Volumenkraft auf die Flüssigkeit größer. Jedoch wird auch die Reichweite des Schallfeldes und der Bereich in dem die Flüssigkeit beschleunigt wird kürzer. Bei dieser Anregung und Geometrie liegt die maximal erreichte Geschwindigkeit bei einer Frequenz zwischen $400 \mathrm{MHz}$ bis $500 \mathrm{MHz}$. 


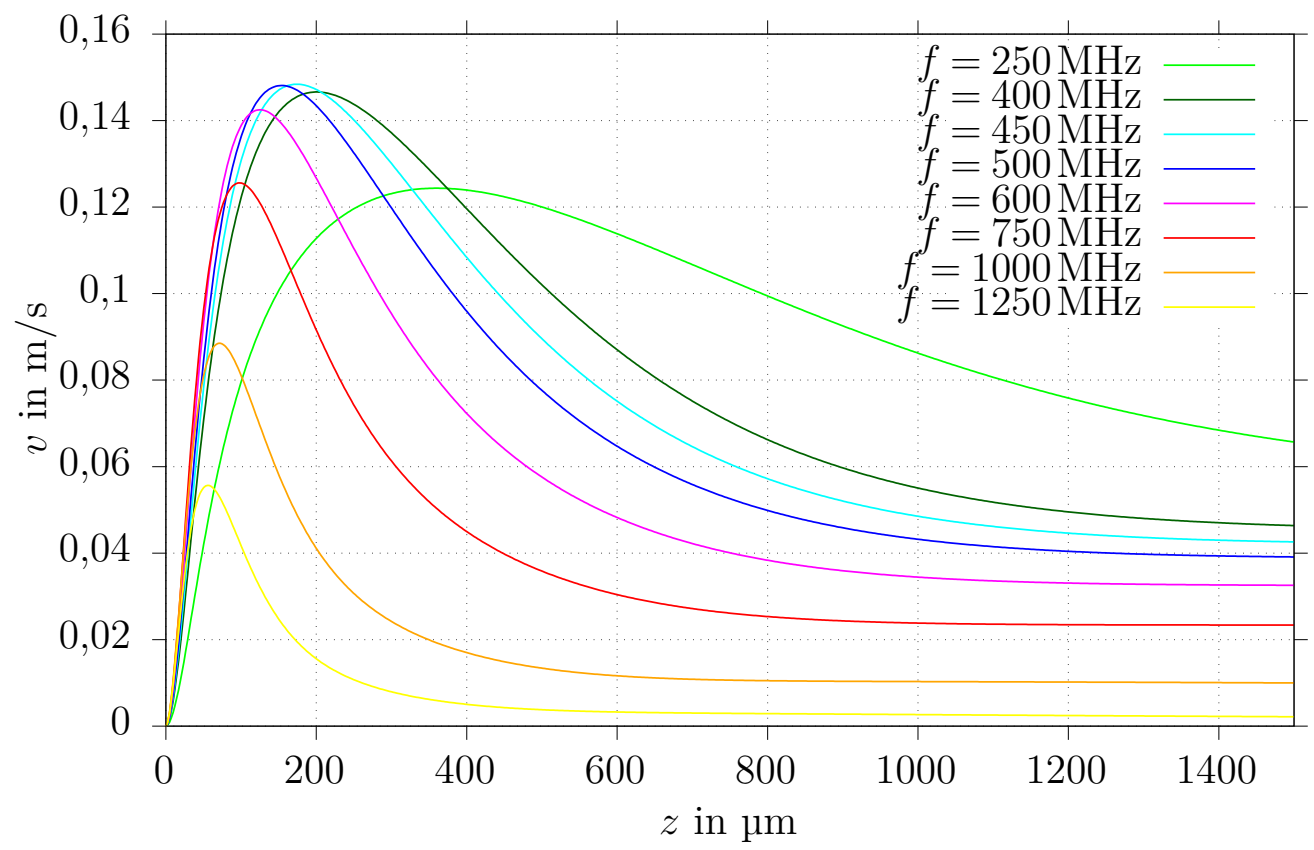

Abb. 3.13.: Strömungsgeschwindigkeit auf der z-Achse für $t \rightarrow \infty$. Die Transducer-Wand-Geschwindigkeit, die zur Berechnung der Volumenkraft durch ein linear dissipierendes Schallfeld genutzt wurde, ist für alle Frequenzen $u_{0}=0,5 \mathrm{~m} / \mathrm{s}$

\subsubsection{Strömung durch Volumenkraft eines nichtlinearen Schallfeldes}

Die obere Näherung ist nur für niedrige Amplituden gültig. Bei einer Frequenz von $750 \mathrm{MHz}$ wird es ab einer Partikelgeschwindigkeit von $u_{0} \approx 1,5 \mathrm{~m} / \mathrm{s}$ nötig, das Schallfeld und die daraus resultierende Volumenkraft mit der nichtlinearen Näherung aus Abschnitt 2.7.4 zu berechnen.

Die maximale Strömungsgeschwindigkeit ist nicht mehr bei $t \rightarrow \infty$ zu finden, sondern gerade bei stärkerer Anregung bereits während sich das Strömungsfeld ausbildet. Durch einen Anfahrwirbel, der sich zu beginn der Strömung bildet, wie von [42], [43], [44], [45], [46] beschrieben, und im weiteren zeitlichen Verlauf in $z$-Richtung aus dem simulierten Bereich wandert, kommt es zu höheren Strömungsgeschwindigkeiten. Abbildung 3.14 zeigt die stationäre Strömung, die sich bei einer Schallschnelle von $u_{0}=10 \mathrm{~m} / \mathrm{s}$ nach circa $1 \mathrm{~ms}$ ausbildet. Eine jetförmige Strömung breitet sich in Abstrahlrichtung des Schallwandlers aus. Die höchste stationäre Strömungsgeschwindigkeit befindet sich im Zentrum der Strömung in einem Abstand von $126 \mu \mathrm{m}$ vom Schallwandler. 


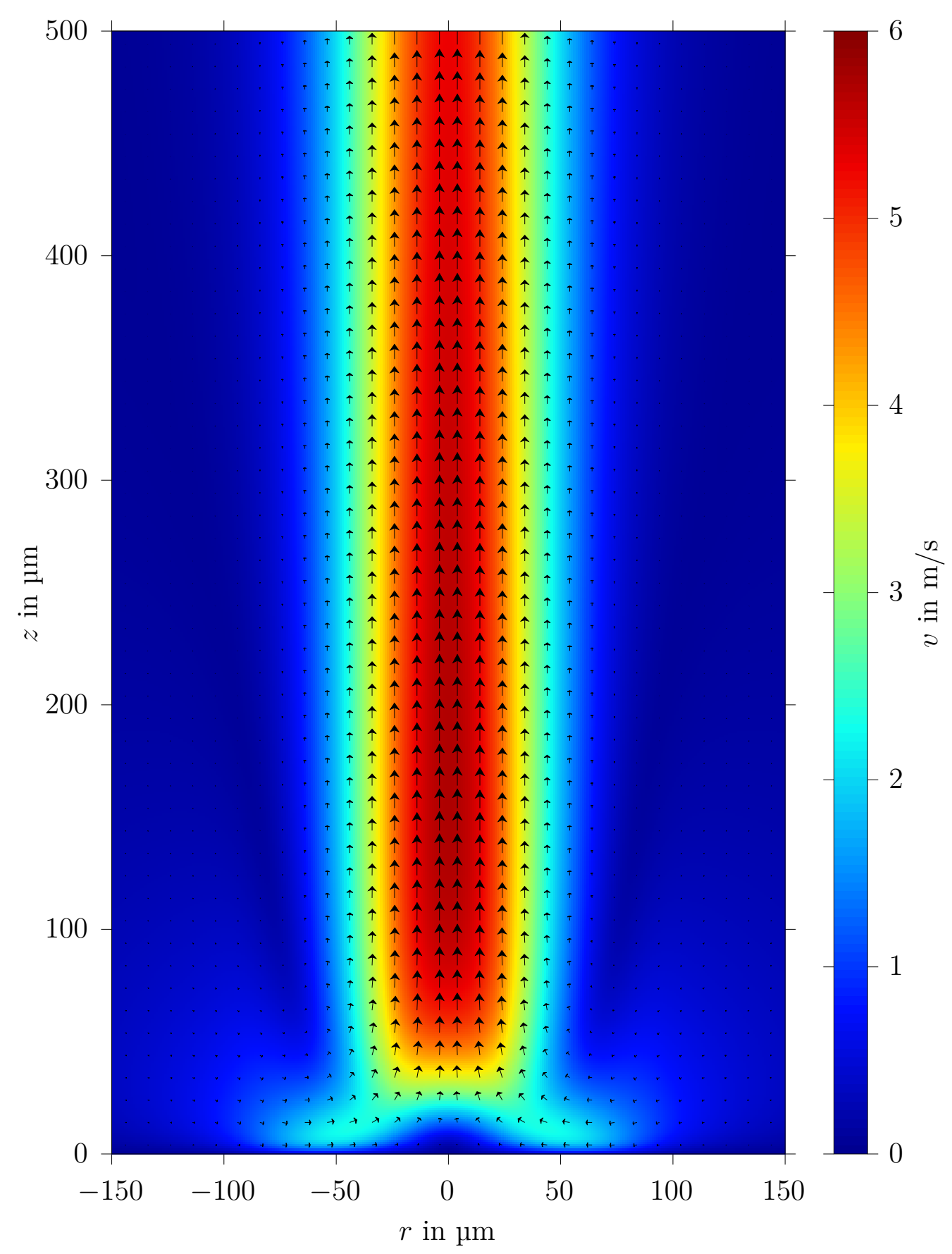

Abb. 3.14.: Der Betrag der Strömungsgeschwindigkeit ist durch die Farbskala wiedergegeben. Zusätzlich zeigen die Pfeile die Strömungsrichtung an. Als treibende Kraft wird die Volumenkraft aus Abbildung 3.10 verwendet, die aus einem nichtlinearen Schallfeld mit $u_{0}=10 \mathrm{~m} / \mathrm{s}$ und $f=750 \mathrm{MHz}$ resultiert.

Zunächst kommt es zur Bildung eines Anfahrwirbels, wie in Abbildung 3.15zu erkennen ist. Der Wirbel läuft dann nach oben aus dem Simulationsgebiet und 


\section{Simulationen}

dahinter bildet sich die stationäre Jetströmung aus. Die höchste Strömungsgeschwindigkeit in der Simulation kann im Zentrum des Wirbels abgelesen werden, als es einen Abstand von $183 \mu \mathrm{m}$ vom Schallwandler hat.

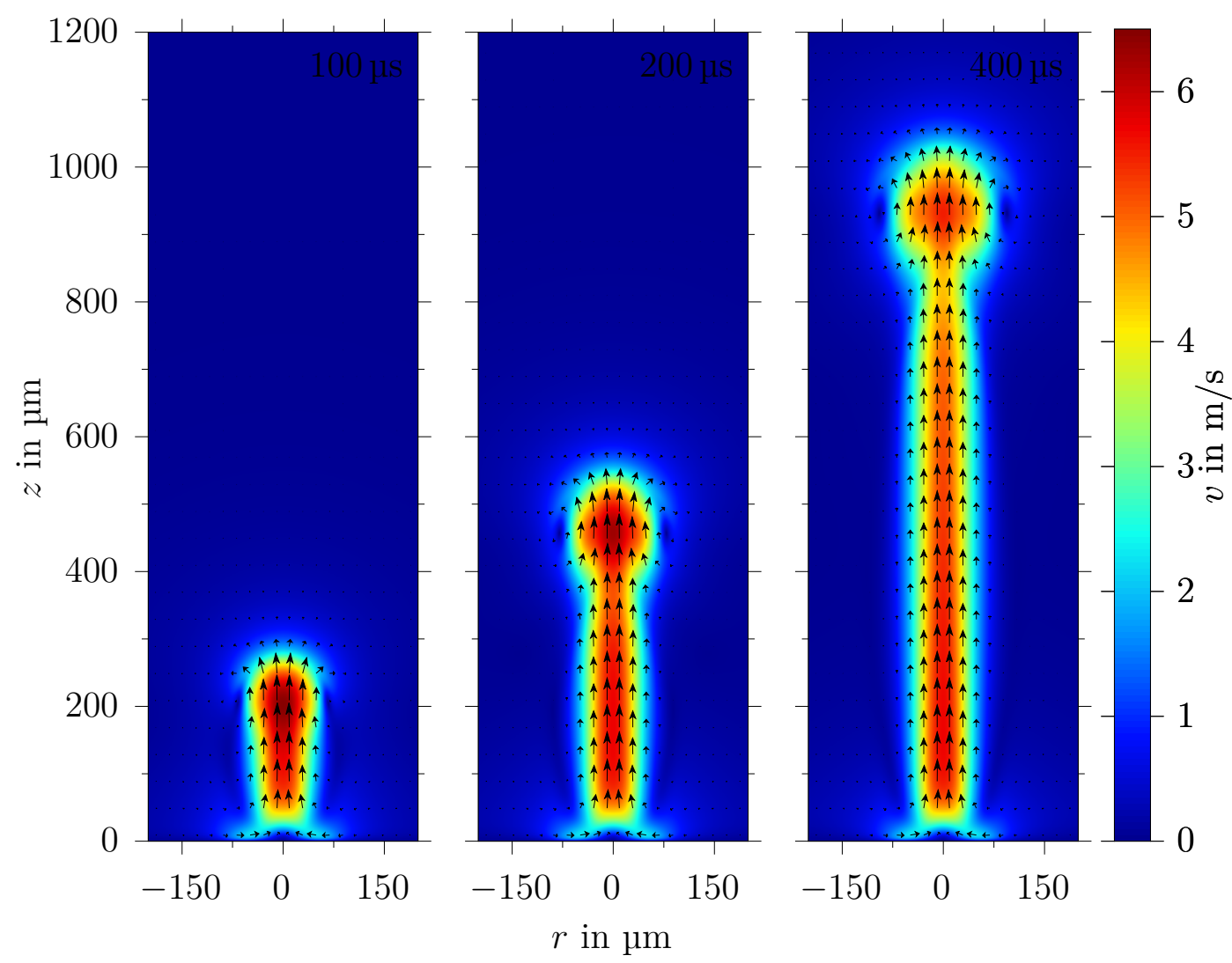

Abb. 3.15.: Ausbreitung der Strömung, der Zeitpunkt $t$ ist rechts oben in den Bildern angegeben. Die Schallschnelle des nichtlinearen Schallfelds die zur Berechnung der Volumenkraft genutzt wurde, ist wie bei Abb. $3.16 u_{0}=10 \mathrm{~m} / \mathrm{s}$ bei einer Frequenz von $f=750 \mathrm{MHz}$

Durch Auftragen der Strömungsgeschwindigkeit auf der Rotationsachse, also auf der z-Achse, für unterschiedliche Zeiten, wie es für das linear dissipierende Schallfeld in Abb. 3.12 getan wurde, ergibt sich Abbildung 3.16

Der Anfahrwirbel ist auch hier durch ein (lokales) Strömungsgeschwindigkeitsmaximum zu erkennen. 


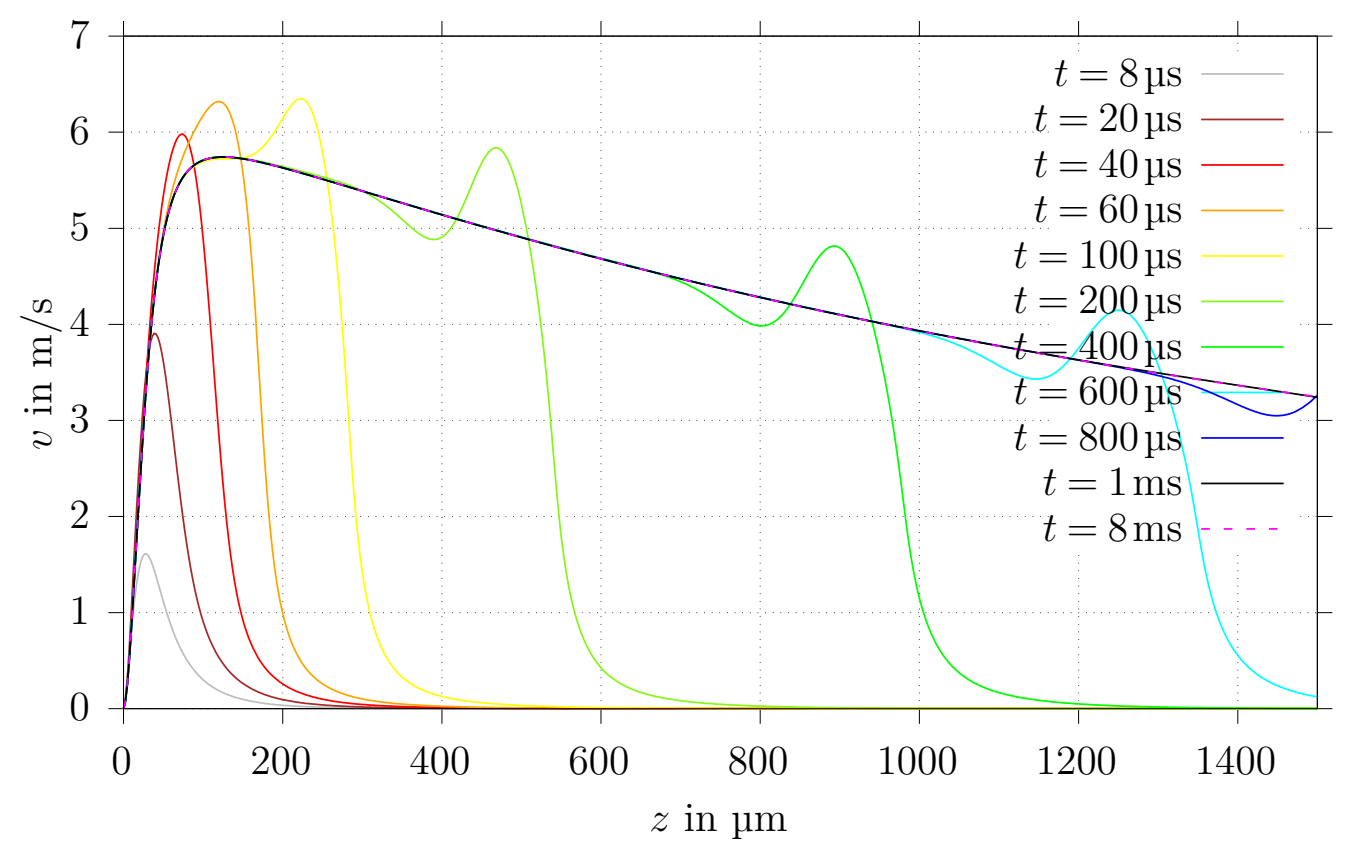

Abb. 3.16.: Strömungsgeschwindigkeit auf der z-Achse für unterschiedliche Zeiten. Die Schallschnelle des nichtlinearen Schallfelds die zur Berechnung der Volumenkraft genutzt wurde, ist $u_{0}=10 \mathrm{~m} / \mathrm{s}$ bei einer Frequenz von $f=750 \mathrm{MHz}$

Die gezeigte Strömung reicht im Gegensatz zum Schallfeld bis weit über das Simulationsgebiet hinaus.

Die hier erwähnten Effekte wie Einschwingzeit, Strömungsgeschwindigkeit und Reichweite der Strömung sind von der Schallfeldamplitude abhängig und werden im Folgenden noch genauer betrachtet.

Die maximal erreichte Strömungsgeschwindigkeit $v_{\max }$ sowie die maximale stationäre Strömungsgeschwindigkeit $v_{e}$ unterscheiden sich mit zunehmender Amplitude. Durch einen Anfahrwirbel ist die Strömungsgeschwindigkeit zunächst lokal etwas größer. Die experimentell beobachteten Anfahrwirbel passen recht gut mit den simulierten überein, wie in Abschnitt 4.2.2 gezeigt wird. 

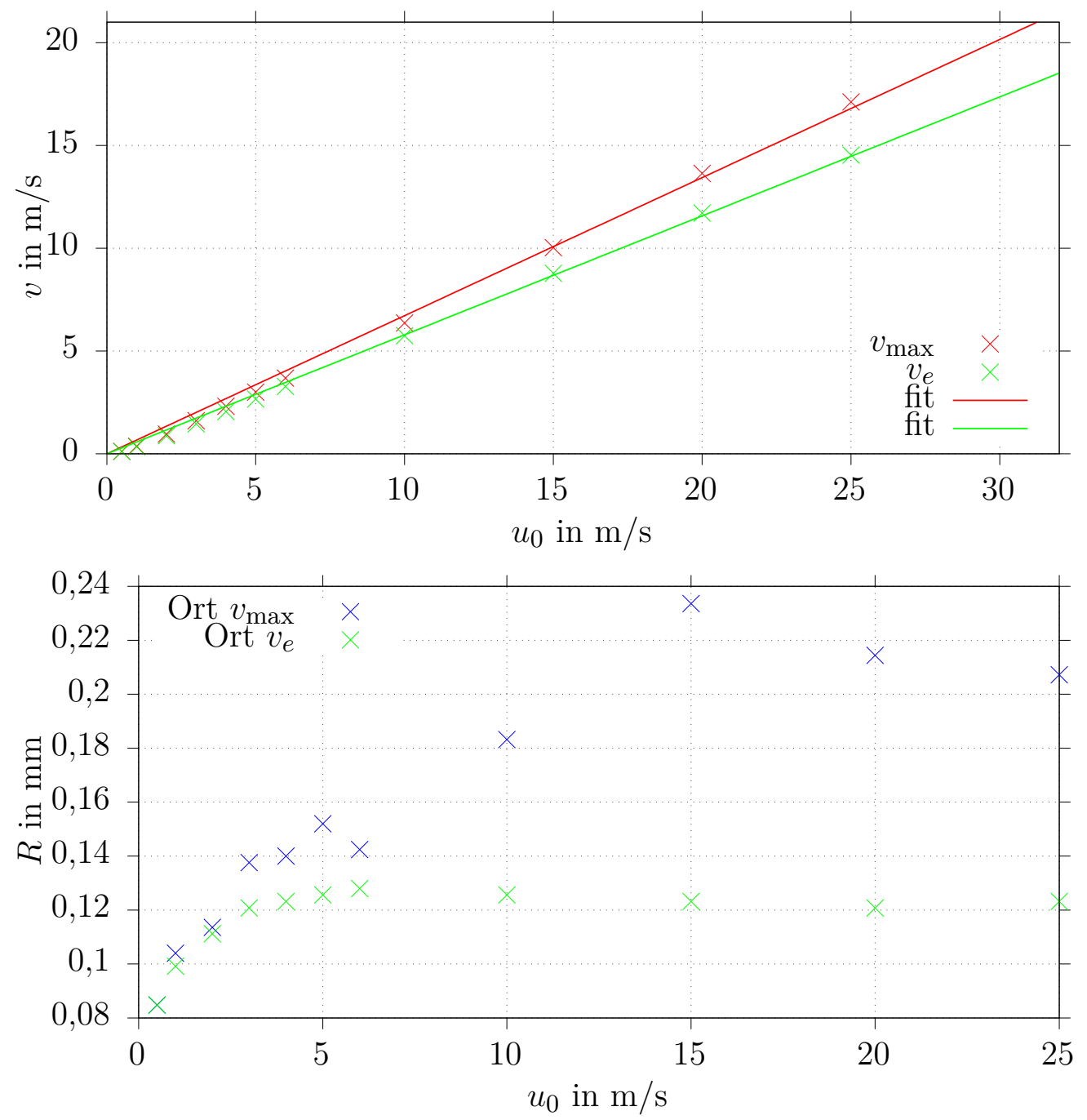

Abb. 3.17.: Maximale Strömungsgeschwindigkeit $v_{\max }$ sowie maximale stationäre Strömungsgeschwindigkeit $v_{e}$ für unterschiedliche Amplituden (oben), mit linearem Fit. Position von $v_{\max }$ und $v_{e}$ für unterschiedliche Amplituden (unten).

In Abbildung 3.18 ist die „Einschwingzeit“ der Strömung für unterschiedliche Schallfeldamplituden angegeben. Für die Einschwingzeit wird nur die maximale Strömungsgeschwindigkeit sowie der Ort des Maximums berücksichtigt. Das Maximum wird als stationär angenommen, wenn die Änderung der Geschwindigkeit pro Zeitschritt unter 0,1\% der maximalen Geschwindigkeit fällt. 


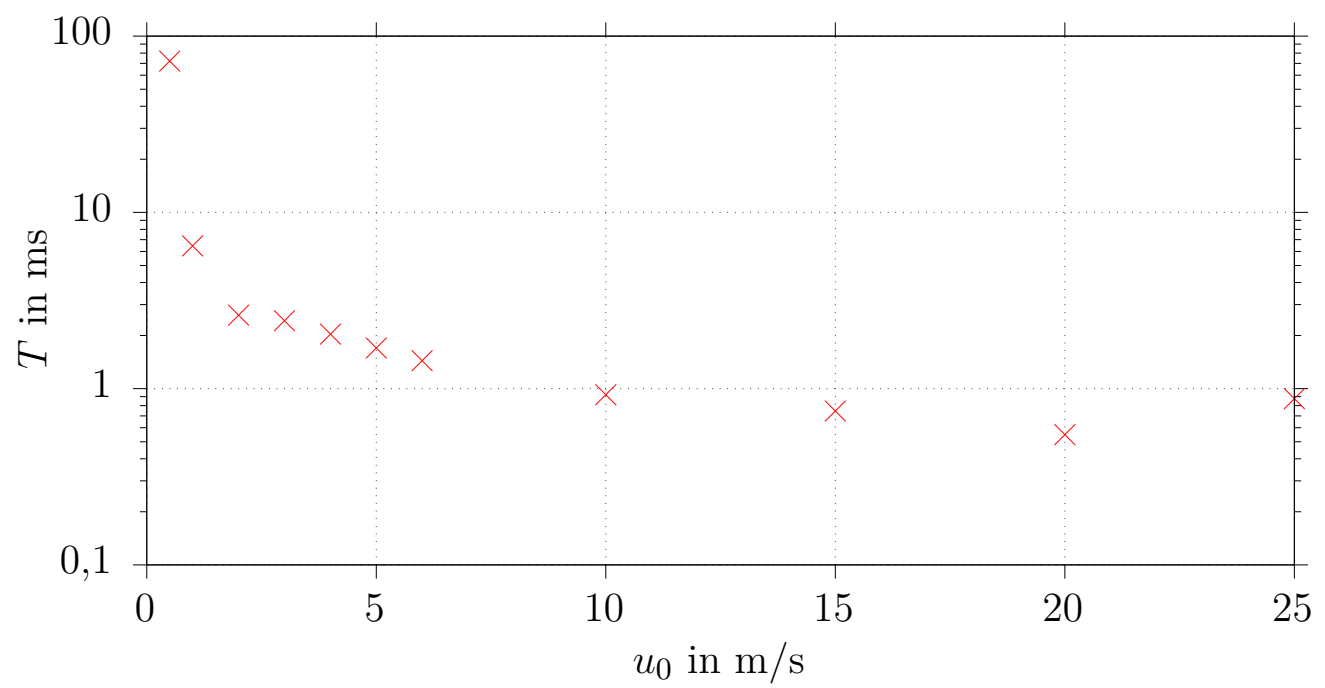

Abb. 3.18.: Einschwingzeit des stationären Strömungsmaximums

Mit steigender Amplitude verkürzt sich die Einschwingzeit und die Reichweite der Strömung vergrößert sich, wie im Graph 3.19 abgebildet. Als Reichweite wird hier der Wert bezeichnet, bei dem die stationäre Strömung auf der zAchse noch eine Amplitude von $v_{\mathrm{e}} / e$ hat.

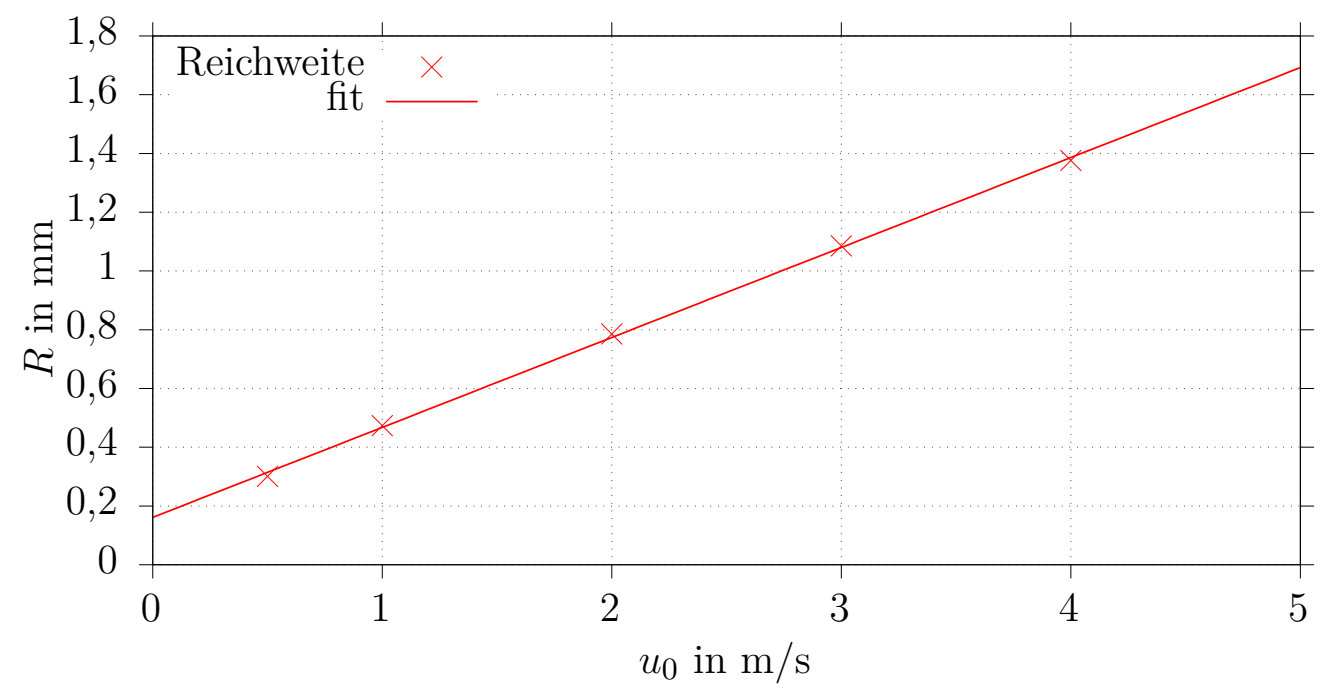

Abb. 3.19.: Reichweite der Strömung

Bereits ab einer Partikelgeschwindigkeit $u_{0} \approx 7 \mathrm{~m} / \mathrm{s}$ liegt die Reichweite der Strömung außerhalb des simulierten Bereichs $(z<2 \mathrm{~mm})$, daher gibt es für die Reichweite weniger Datenpunkte.

Die Volumenkraft und damit die resultierende Strömung ist von der Amplitude des Schallfeldes und auch von der Frequenz abhängig. Die Abbildung 3.20 


\section{Simulationen}

zeigt die Strömungsgeschwindigkeit auf der Rotationsachse für eine Amplitude von $u_{0}=10 \mathrm{~m} / \mathrm{s}$ für unterschiedliche Frequenzen im stationären Zustand.

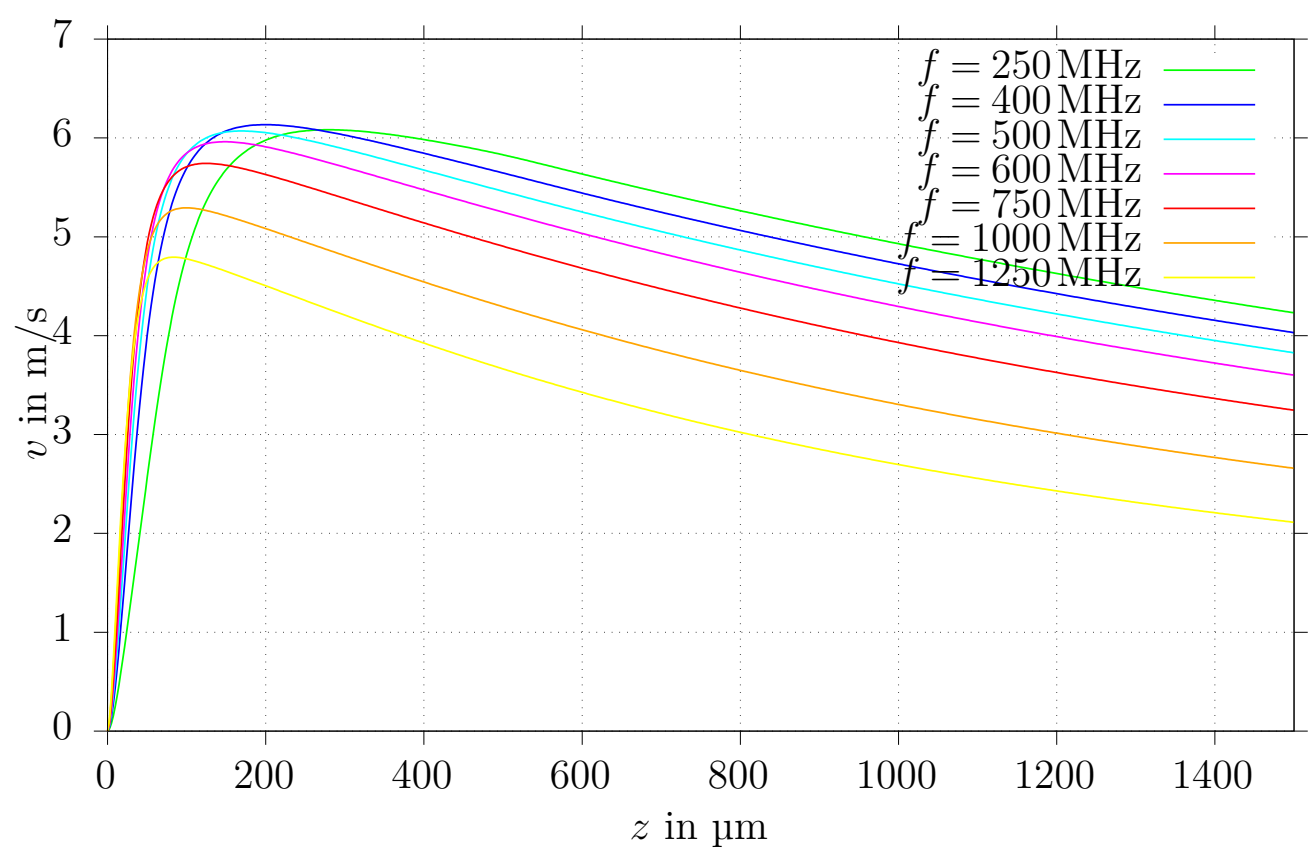

Abb. 3.20.: Strömungsgeschwindigkeit auf der z-Achse für unterschiedliche Frequenzen. Die Partikelgeschwindigkeit, die zur Berechnung der Volumenkraft genutzt wurde, ist $u_{0}=10 \mathrm{~m} / \mathrm{s}$

Durch die konstante Amplitude ändert sich mit der Frequenz die $R e_{\text {ak }}$ wie in der Tabelle 3.2 notiert.

Tab. 3.2.: Aksutische Reynoldszahl $R e_{\mathrm{ak}}$ der Simulationen aus Abb. 3.20

\begin{tabular}{c|c|c|c|c|c|c|c|c}
\hline \hline für & \multicolumn{8}{|c}{$f$ in MHz } \\
$u_{0}=10 \mathrm{~m} / \mathrm{s}$ & 250 & 400 & 450 & 500 & 600 & 750 & 1000 & 1250 \\
\hline$R e_{\mathrm{ak}}$ & 19,47 & 12,17 & 10,82 & 9,73 & 8,11 & 6,49 & 4,87 & 3,89 \\
\hline
\end{tabular}

Bei den niedrigeren Frequenzen ist bei gleicher Amplitude die akustische Reynoldszahl größer, also kommt es zu einer stärkeren Nichtlinearität, was die effektive Dissipationslänge verkürzt. Hierdurch werden die resultierenden Strömungen der unterschiedlichen Frequenzen ähnlicher, verglichen mit dem linearen Fall in Abbildung 3.13 . 


\section{Experimente}

Bei den Experimenten wurden neuartige Schallwandler verwendet, die nahe einem Gigahertz arbeiten. Diese hohen Frequenzen die im UHF-Band (ultra high frequency) von $300 \mathrm{MHz}$ bis $3000 \mathrm{MHz}$ liegen, die ansonsten eher bei Mobilfunkübertragungen Verwendung finden, stellen besondere Anforderungen. Durch die Verbreitung dieses Frequenzbereiches unter Anderem bei der kabellosen Datenübertragung sind Geräte wie Funktionsgeneratoren, Verstärker und Richtkoppler erhältlich.

Bei dieser Hochfrequenztechnik müssen Effekte, wie die Wellenlänge im Koaxialkabel, die bei der Erzeugung von Ultraschall in der Regel vernachlässigt werden, doch beachtet werden [47]. Alle gezeigten Experimente sind in DI-Wasser durchgeführt mit je nach Experiment variierenden Zusätzen von Tinte, Partikeln oder Salzen, die jeweils für die Strömungsmessung benötigt werden.

\subsection{Wandler}

Die in dieser Arbeit verwendeten Schallwandler wurden vom Fraunhofer-Institut für Biomedizinische Technik IBMT in St. Ingbert entwickelt. Hierbei handelt es sich um Wandler, die mit einer Kolbenbewegung arbeiten. Die Resonanzfrequenzen der Wandler liegen zwischen $600 \mathrm{MHz}$ bis $800 \mathrm{MHz}$. Die Resonanzen variieren je nach Wandler und nur einige der Resonanzen sind für den Betrieb geeignet.

\subsubsection{Aufbau}

Bei der Herstellung werden mehrere Schichten auf einem Siliziumwafer abgeschieden. Der Schichtaufbau für einen einzelnen Wandler ist in Abbildung 4.1 skizziert.

Es wurden mehrere Arten von Wandlern hergestellt. Dabei wurde unterschiedliches Elektrodenmaterial verwendet sowie die Dicke des Siliziumwafers variiert. Bei der ersten Generation der Schallwandler bestehen die Elektroden aus Gold mit einer Schicht Chrom als Haftvermittler. Zusätzlich sind mehrere Wandler auf einem Chip in Reihen- sowie Parallelschaltung ausgeführt. Bei den weiteren Generationen werden nur Einzelwandler getestet. 


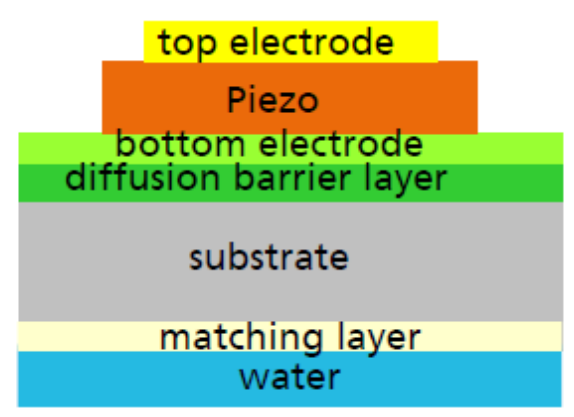

Abb. 4.1.: Schichtaufbau des Schallwandlers. Quelle: Fraunhofer IBMT

Um die Probleme, die im Folgenden skizziert werden, zu verringern, wurde bei der zweiten Generation die Elektrode aus Platin mit Titan als Haftvermittler verwendet; der weitere Aufbau ist identisch zur ersten Generation. Die dritte Generation unterscheidet sich durch die Dicke des Siliziums, welches zu einer dünnen Membran geätzt wurde. Das Piezomaterial ist bei allen Wandlern Zinkoxid.

\subsubsection{Messungen am Fraunhofer IBMT}

An den Wandlern sind bereits vom Fraunhofer IBMT Messungen zur Resonanzfrequenz, Impedanz und Schwingungsverhalten durchgeführt worden.

Die Auslenkung des Schallwandlers ist in Abbildung 4.2 gezeigt.

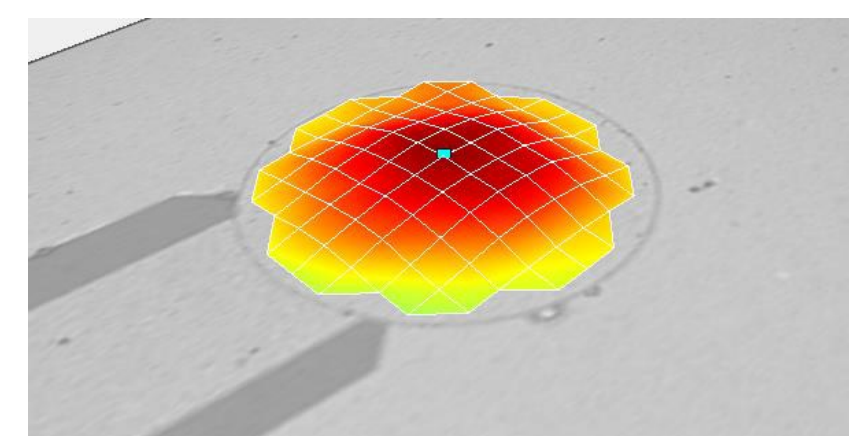

Abb. 4.2.: Vibrometermessung der Auslenkung, farbcodiert, Quelle: Fraunhofer IBMT

Es ist gut die erste Oberflächenmode zu erkennen, die einer Dickenschwingung mit zentralem Maximum entspricht.

Die frequenzabhängige Phasenverschiebung sowie der Widerstand der Schallwandler wurde ebenfalls vom Fraunhofer IBMT vermessen und wird in der Abbildung 4.3 exemplarisch für einen Schallwandler gezeigt. 


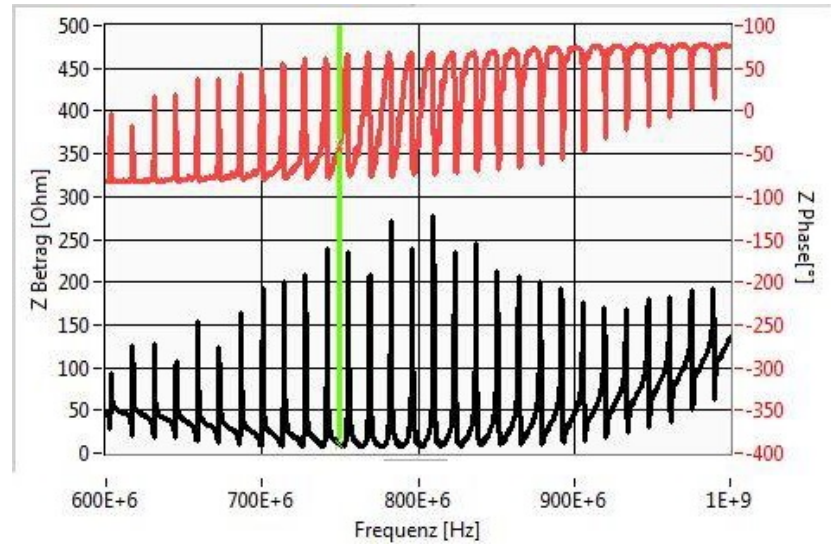

Abb. 4.3.: Widerstand und Phase eines Wandlers, Quelle: Fraunhofer IBMT

Die Kurven sind für jeden Wandler leicht unterschiedlich, daher muss bei den Experimenten für jeden Schallwandler zunächst eine gut funktionierende Resonanzfrequenz gefunden werden.

\subsubsection{Probleme}

Die verwendeten Schallwandler weisen bei zu hoher Beanspruchung zwei unterschiedliche Arten an Defekten auf. In Abbildung 4.4 ist exemplarisch ein Wandler mit beiden Defekten gezeigt.

Der Kreis in Abbildung 4.4 stellt die obere Elektrode dar. Im Zentrum der Elektrode sind dunkle Bereiche zu erkennen, die durch Zerstörung der Elektrode entstanden sind. Die Zerstörung entsteht vermutlich durch die Auslenkung des Piezomaterials. Zum Test wird der Wandler abseits der Resonanzfrequenz betrieben und die Spannung gesteigert, bis der erste Schaden zu erkennen ist. Derselbe Schaden tritt bei der Resonanzfrequenz bereits bei deutlich niedrigeren Spannungen auf. 


\section{Experimente}

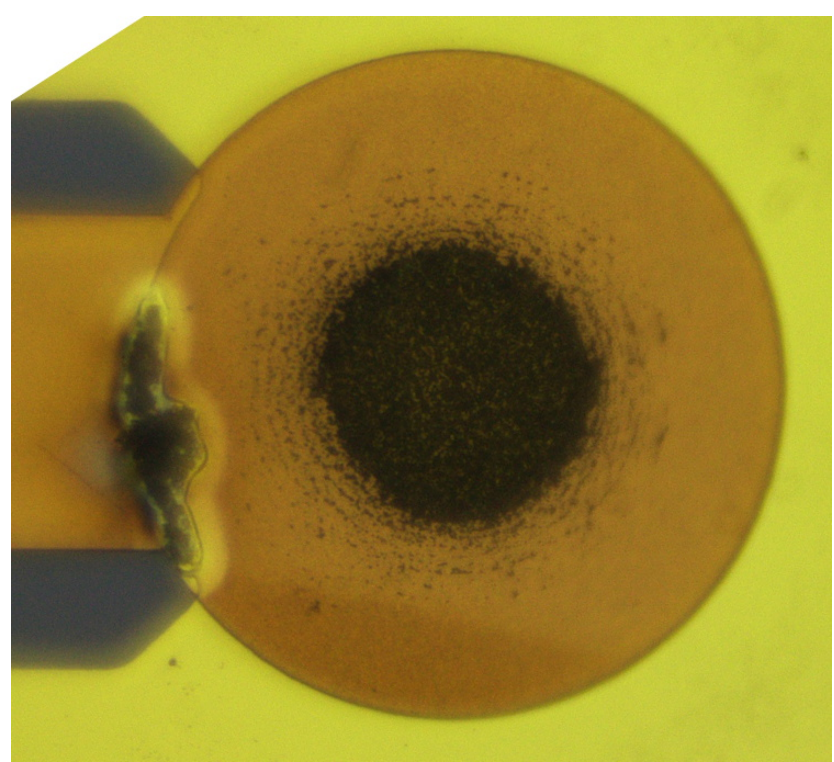

Abb. 4.4.: Optisches Bild eines defekten Schallwandlers, Ansicht von oben nach Abb. 4.1

Da die Strukturen teilweise unter der optischen Auflösungsgrenze liegen, werden detaillierte Aufnahmen mittels eines Raster-Elektronen-Mikroskops (REM) gemacht. Ein Beispiel ist in Abb. 4.4 gezeigt.

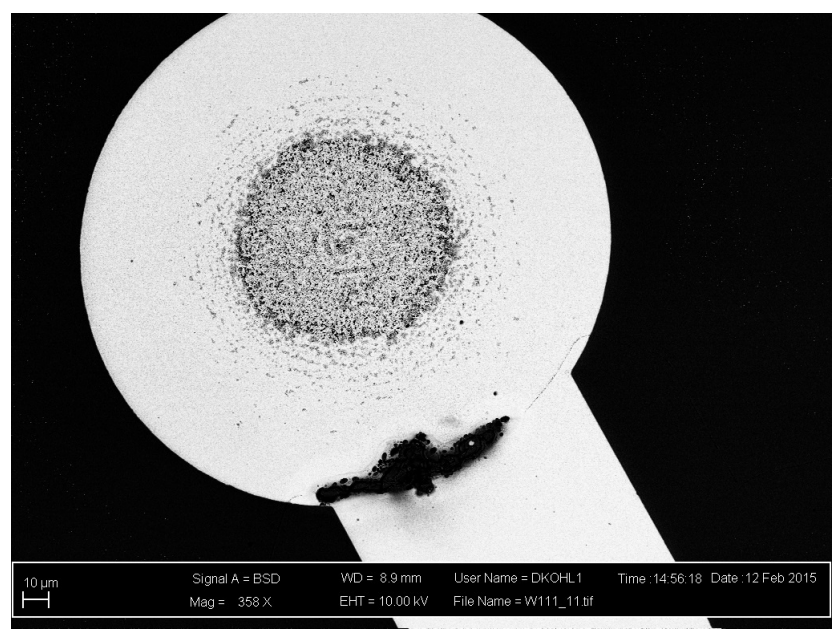

Abb. 4.5.: REM Bild eines defekten Schallwandlers -Übersicht

Abbildung 4.6 zeigt den Randbereich der zerstörten Elektrode. ebenfalls als REM-Aufnahme. 


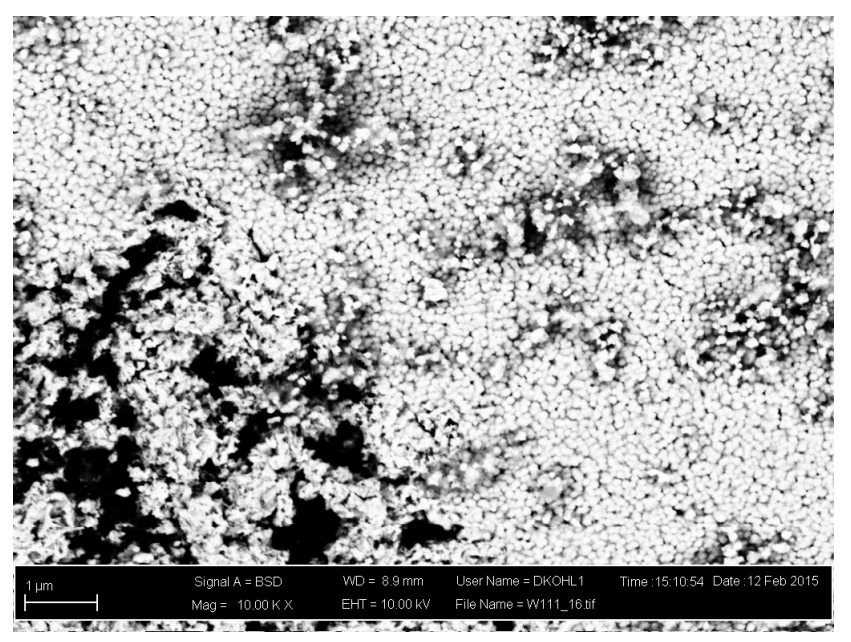

Abb. 4.6.: REM Bild eines defekten Schallwandlers -Detail

Auf der rechten Hälfte des Bildes ist die noch intakte Elektrode mit den aufgewachsenen Goldinseln zu erkennen. Am linken Rand haben sich diese Goldinseln abgelöst und wurden über die Oberfläche der Elektrode verteilt. Der zweite Mechanismus, der den Wandler zerstört, ist vermutlich ein Spannungsdurchschlag oder ein Überhitzen der Anschlussstelle der oberen Elektrode an die Leiterbahn. Bei zu hoher Spannung sowie längerer Laufzeit des Schallwandlers und damit steigender Temperatur kann es zu dem Schaden am Rand der Elektrode kommen. Auch von diesem Schadensbild gibt es eine REM Aufnahme, die in Abbildung 4.7 zu sehen ist.

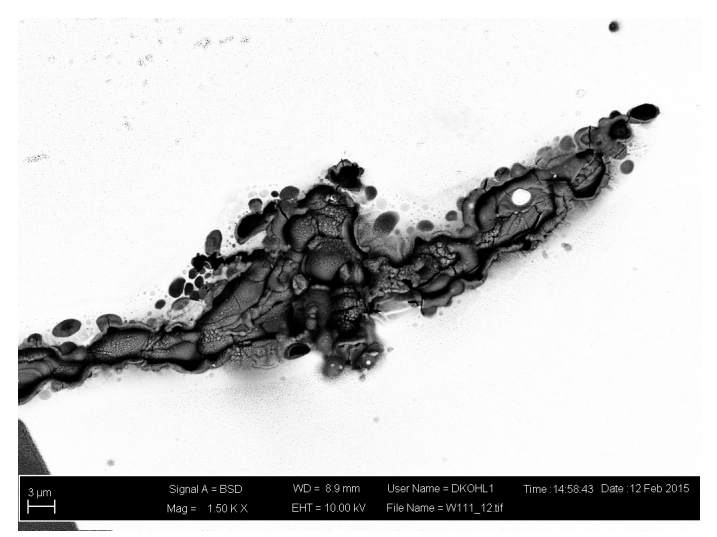

Abb. 4.7.: REM Bild eines defekten Schallwandlers -Detail

Da die Anzahl der Schallwandler stark limitiert ist und die Belastungsgrenzen der Wandler stark variieren, können keine detaillierten Aussagen über die Belastungsgrenzen gemacht werden. Einzelne Wandler haben Spannungen (Spitze-Spitze bzw. peak-peak) von $U_{p p}=80 \mathrm{~V}$ überstanden bei Betriebsdauern im Bereich von einigen ms. 


\subsection{Strömungsmessung mit Tinte}

Für die Visualisierung der Strömung eignet sich Tinte, bzw. die Grenze des eingefärbtem Bereichs. Dazu wird zunächst die Tinte mit einer Spritze vorsichtig auf den Boden der weiterhin mit DI-Wasser gefüllten Küvette platziert. Die durch den Schallwandler erzeugte Strömung bzw. Bewegung der Tintenfront wird mit einer Hochgeschwindigkeitskamera in der Seitenansicht aufgenommen.

\subsubsection{Versuchsaufbau „Tinte“}

Die Ansteuerung der Schallwandler sowie der Aufbau zur optischen Messung sind in Skizze 4.8 dargestellt und die verwendeten Geräte in Tabelle 4.1 aufgelistet.

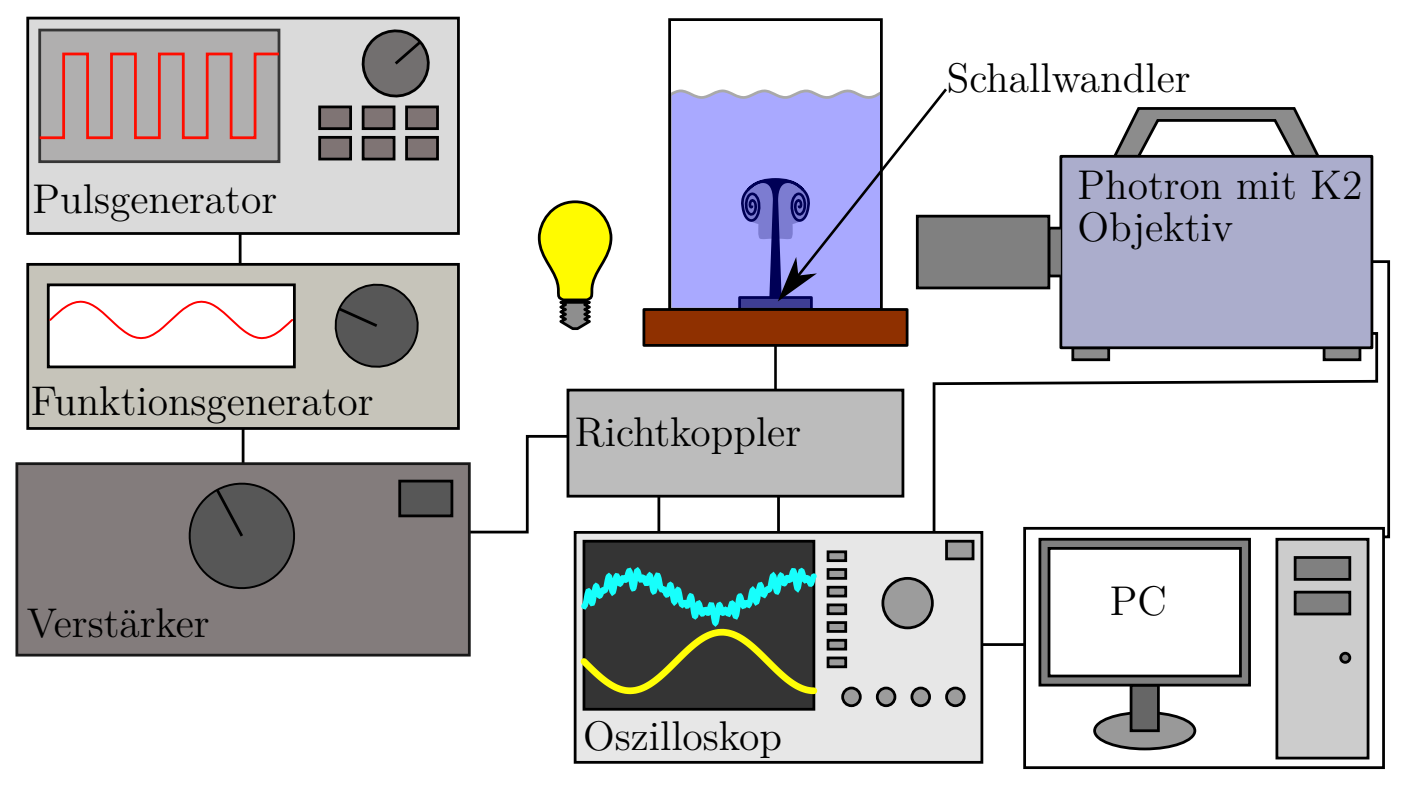

Abb. 4.8.: Versuchsaufbau

Tab. 4.1.: Verwendete Geräte für den Versuchsaufbau „Tinte“

\begin{tabular}{ll}
\hline \hline Pulsgenerator & Tektronix AFG 3021 \\
Funktionsgenerator & Hameg HM 8135 \\
Verstärker & ar 15S1G4 \\
Richtkoppler & Mini-Circuits ZABDC20-182H+ \\
Oszilloskop & Tektronix DPO 7254 \\
Beleuchtung & Nikon D-LH/LC HALOGEN 12V100W \\
Kamera & Photron Fastcam SA5 \\
Objektiv & Infinity K2 CF3
\end{tabular}


Als Küvette wird eine PMMA Einmalküvette der Firma Roth mit einer Höhe von $45 \mathrm{~mm}$ sowie einer Breite und Tiefe von 12,5 mm außen bzw. $10 \mathrm{~mm}$ innen verwendet. Zum Montieren der Küvette auf der Platine des Schallwandlers wird der Boden der Küvette abgesägt und die vier transparenten Seiten mit Silikon aufgeklebt.

Die Ansteuerung der Schallwandler erfolgt über einen Pulsgenerator, der ein TTL-Signal erzeugt, das den Ausgang des Funktionsgenerators schaltet. Der Ausgang des Funktionsgenerators, der das hochfrequenten Sinussignal erzeugt, wird dann über den Verstärker zum Schallwandler geleitet. Zur Messung der Spannung am Schallwandler wird ein Richtkoppler zwischen Verstärker und dem Schallwandler eingesetzt. Dies ermöglicht die Messung der angelegten Spannung am Wandler durch ein Oszilloskop.

\subsubsection{Messung Tinte}

Abbildung 4.9 zeigt einen Ausschnitt aus einer Hochgeschwindigkeitsaufnahme. Durch Abziehen eines Referenzbildes wird nur die bewegte Tinte sichtbar. Die Tinte, die den Boden der Küvette bedeckt, wird ausgeblendet. Der Schallwandler wurde mit einer Frequenz von $f=745,88 \mathrm{MHz}$ für $1 \mathrm{~ms}$ mit einer Wiederholrate von $10 \mathrm{~Hz}$ betrieben. Jeder Puls erzeugt eine pilzförmige Strömungsfront („Tinten-Pilz“).

Die Strömung direkt oberhalb des Wandlers ist durch die Tinte verdeckt, jedoch kann die Ausbreitung der Tintenfront verfolgt werden. Die Abbildung 4.10 zeigt die Position sowie die ermittelte Geschwindigkeit der Tintenfront bei einer Wandlerspannung von $26 \mathrm{~V}_{\mathrm{pp}}$.

Mit der Simulationsmethode aus Abschnitt 3.5 wird versucht, die experimentellen Daten nachzustellen. 


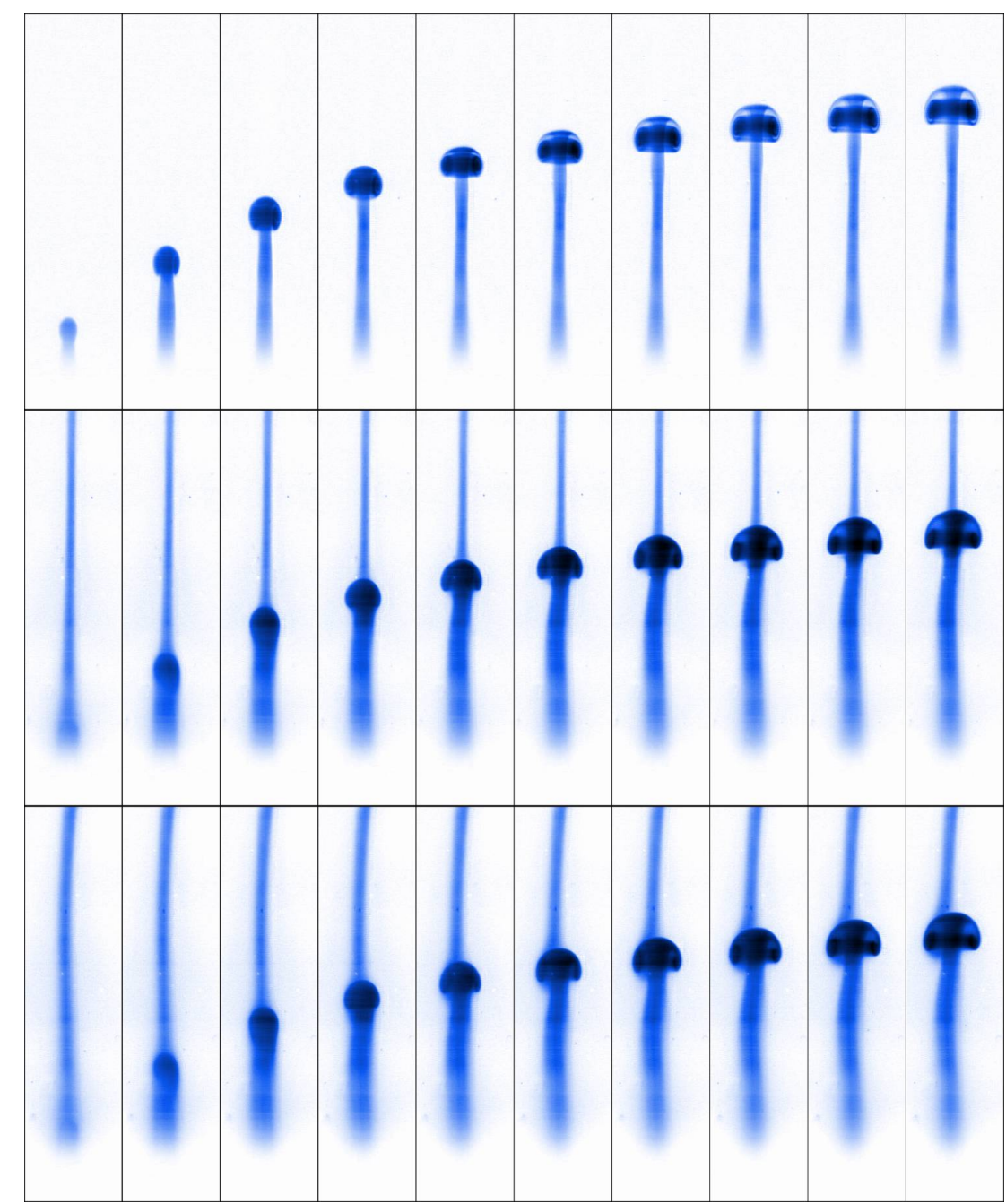

Abb. 4.9.: Aufnahmen der Strömung durch (Tinten-Pilze). Eingefärbte s/wAufnahmen und durch Abziehen eines Referenzbildes bereinigt. Die erste Reihe zeigt den ersten Tinten-Pilz, die zweite Reihe den zweiten und die dritte Reihe den dritten. Der Schallwandler wird mit $10 \mathrm{~Hz}$ für $1 \mathrm{~ms}$ betrieben. Die Zeit zwischen zwei Bildern in einer Reihe ist $0,75 \mathrm{~ms}$ und $100 \mathrm{~ms}$ in einer Spalte. Eine Aufnahme zeigt einen Bereich von 4,12 x 1,02 $\mathrm{mm}^{2}$. Der Schallwandler befindet sich mittig am unteren Bildrand.

Der einzige verwendete Parameter ist die Partikelgeschwindigkeit $u_{0}$ am Schallwandler, die die Volumenkraft vorgibt. Die anderen Parameter wie Frequenz und Materialeigenschaften von Wasser sowie die Füllhöhe der Tinte werden durch das Experiment vorgegeben. 

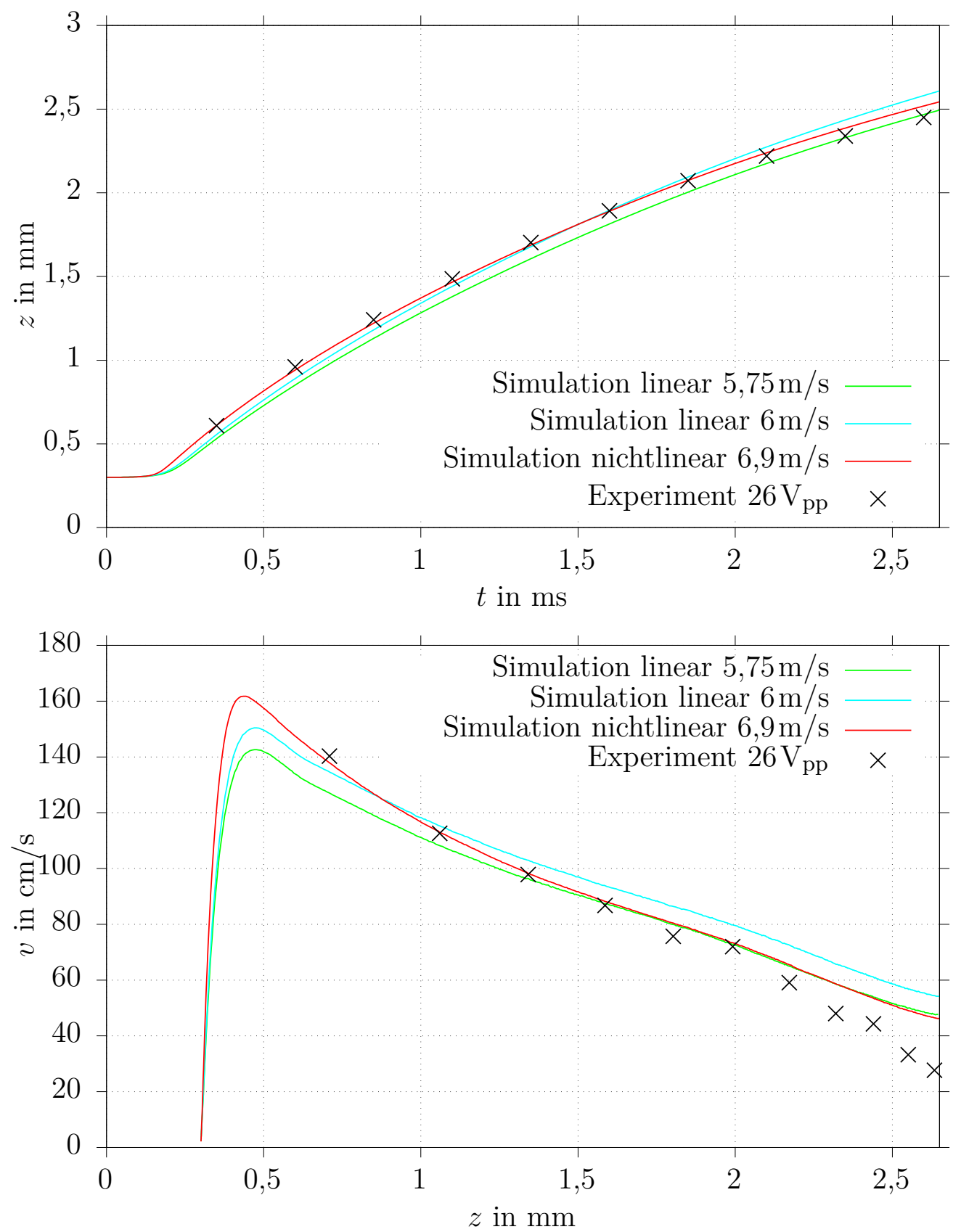

Abb. 4.10.: Ausbreitung der Tintenfront aus Abbildung 4.9 mit passender Simulation als ebene Welle in linearer und nichtlinearer Näherung. Oben: Position der Tintenfront gegen die Zeit. Unten: Geschwindigkeit der Tintenfront gegen den Abstand vom Wandler. Bei den Simulationen ist der Parameter $u_{0}$ in $\mathrm{m} / \mathrm{s}$ angegeben.

Die Simulation, in der die Volumenkraft als eine ebene nichtlineare dissipierende Welle (siehe Abschnitt 3.4.2) mit $u_{0}=6,9 \mathrm{~m} / \mathrm{s}$ genähert wurde, zeigt die beste Übereinstimmung mit dem Experiment. Ein direkter Vergleich der 
Aufnahmen und einer Strömungssimulation mit Comsol ist in Abbildung 4.11 gezeigt.

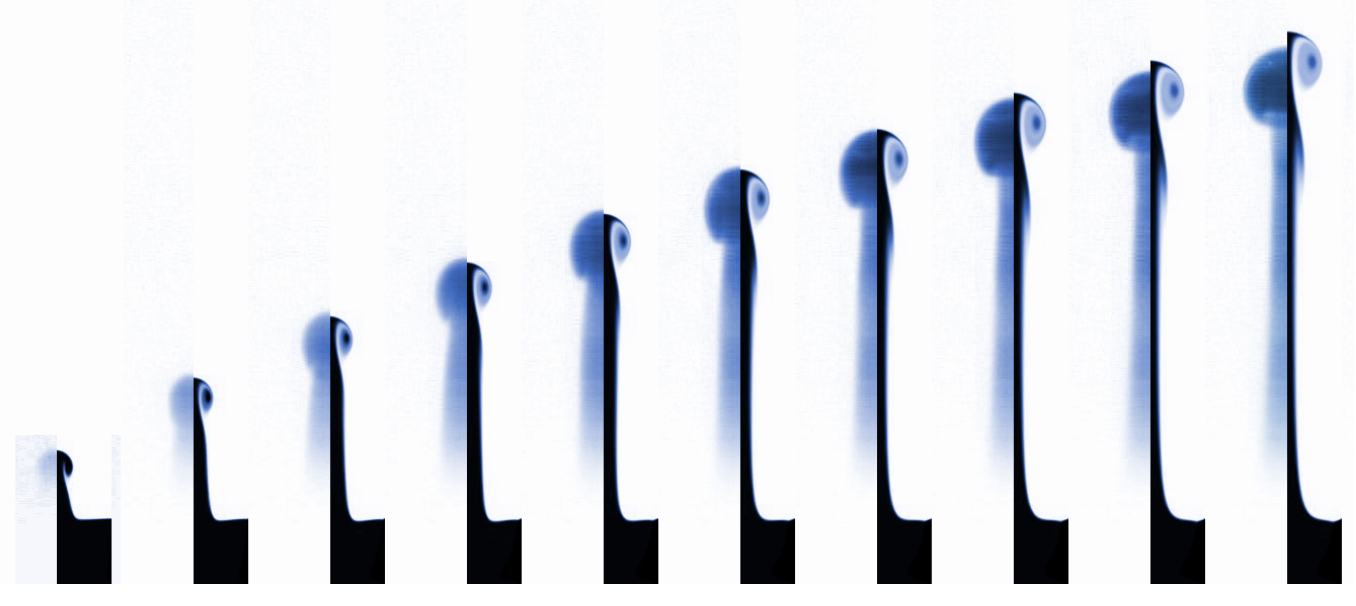

Abb. 4.11.: Vergleich von Simulation (rechts) und Experiment (links) eines Tinten-Pilzes durch akustisch erzeugte Strömung. Die Zeit zwischen zwei Bildern beträgt $0,75 \mathrm{~ms}$. Eine Aufnahme zeigt einen Bereich von $4,12 \times 1,02 \mathrm{~mm}^{2}$.

Aus der Simulation ergibt sich für die Schnelleamplitude am Wandler $u_{0}=$ $6,9 \mathrm{~m} / \mathrm{s}$, folglich eine $R e_{\mathrm{ak}}$ von $\approx 4,43$. Ebenfalls lässt sich aus der Simulation eine maximale Strömungsgeschwindigkeit von $v_{\max }=4,5 \mathrm{~m} / \mathrm{s}$ während des Pulses ermitteln und nähert sich einem $v_{e}$ von $3,84 \mathrm{~m} / \mathrm{s}$ an. Diese Geschwindigkeiten reihen sich in die Simulationen aus Abschnitt 3.5, bei denen mit $750 \mathrm{MHz}$ gerechnet wurde ein.

\subsubsection{Ergebnisse Tinte}

In den Tintenaufnahmen ist die entstehende Strömung qualitativ gut zu erkennen. Durch Einschalten des Wandlers entsteht ein pilzförmiger Anfahrwirbel über dem Wandler, der auch nach Abschalten des Wandlers weiter läuft. Der „Kopf“ der Strömung wird durch einen Ringwirbel gebildet. Der Stiel des Pilzes entsteht durch das nachströmende Fluid.

Schon durch die Größe der „Pilze“ ist zu erkennen, dass die Strömung deutlich weiter reicht als das Schallfeld wirkt, sowohl räumlich als auch zeitlich.

Durch Verfolgen der Tintenfront lässt sich die Ausbreitungsgeschwindigkeit bestimmen, jedoch wird der interessante Bereich direkt vor dem Wandler durch die Tinte selbst verdeckt. Diese ist intransparent und verdeckt damit einen Bereich (je nach Füllhöhe der Tinte von circa $300 \mu \mathrm{m}$ ) über dem Wandler. Ebenso hat die Tinte auch einen Einfluss auf die Strömung, da sie eine geringfügig andere Dichte und Viskosität als Wasser hat. Bei zu hoher Konzentration der Tinte und der damit höheren Dichte gegenüber dem Wasser spielen auch gravitative Effekte eine Rolle. 
Eine quantitative Aussage über die generellen Strömungsgeschwindigkeiten lässt sich nur sehr begrenzt machen, da nur die Ausbreitungsgeschwindigkeit der Tintenfront gemessen werden kann. Jedoch sind schon die Daten der Ausbreitung der Tintenfront ausreichend, um den nichtlinearen Ansatz bei den Simulationen zu unterstützen, da sich ein deutlich bessere Übereinstimmung zwischen Experiment und Simulation ergibt, wenn die Nichtlinearität des Schallfeldes berücksichtigt wird. Bei Tintenpilzen, die mit geringerer Amplitude erzeugt wurden, zeigt auch die lineare Näherung gute Übereinstimmung zum Experiment.

Die Entwicklung des Pilzes wird in der Simulation bis zu einer Höhe von $\approx 2 \mathrm{~mm}$ recht gut wiedergegeben. Ab da ist die Ausbreitungsgeschwindigkeit im Experiment deutlich niedriger als in der Simulation, bei der eine laminare Strömung angenommen wird. Mit einer Reynoldszahl von $R e_{\text {hy }} \approx 900$ bezogen auf $v_{\max }$ und mit dem Durchmesser des Schallwandlers als charakteristische Länge ist auch eine laminare Strömung zu erwarten. Dennoch scheinen viskose Effekte im Wirbel für zusätzliche Dissipation zu sorgen.

Für eine weite Parameterstudie ist das Tintenexperiment zu aufwändig, da nach jedem Puls die Küvette neu mit Tinte präpariert werden muss und sich die meisten Parameter erst durch eine passende Simulation finden lassen.

Für eine bessere räumliche Auflösung wird im nächsten Abschnitt die Tinte durch Partikel ersetzt, die als Tracer im Fluid fungieren.

\subsection{Particle Tracking Velocimetry (PTV)}

Bei der „Particle Tracking Velocimetry“, kurz PTV, werden Tracer-Partikel in das Fluid gegeben. Durch die Partikel, die sich mit der Strömung bewegen, ist es möglich, die Fluidbewegung sichtbar zu machen. Im Gegensatz zur Tinte gibt es keine Bereiche, die überhaupt nicht mehr sichtbar sind. Zusätzlich gibt es für das PTV bereits fertige Algorithmen für die Auswertung der Bewegung der einzelnen Partikel.

\subsubsection{Versuchsaufbau PTV}

Der Versuchsaufbau der PTV-Messung ist fast identisch mit dem Aufbau bei der Tinte mit dem Unterschied, dass die Tinte durch Melanin Resin $10 \mu \mathrm{m}$ 


\section{Experimente}

Partikel ersetzt wird, um die Strömung sichtbar zu machen. Die Wandler werden weiterhin mit den Geräten aus Tabelle 4.1 betrieben und sind, wie in Abb. 4.8 skizziert, aufgebaut.

Bei den Schallwandlern wurden zwei unterschiedliche Betriebsarten vermessen:

- Dauerbetrieb: Der Schallwandler wird für die Dauer der Aufnahme von 100 ms durchgängig betrieben.

- Pulsbetrieb: Der Schallwandler wird mit $25 \mathrm{kHz}$ gepulst mit $50 \%$-Anzeit betrieben; also für immer $20 \mu \mathrm{s}$ „an" gefolgt von derselben Zeit „aus“ über die Dauer der Messung.

Die Schallwandler wurden auch mit unterschiedlichen Schaltungen hergestellt, die an dieser Stelle auch kurz auf ihre Eignung getestet wurden:

- Reihenschaltung von mehreren Schallwandlern

- Parallelschaltung von mehreren Schallwandlern

- einzelner Schallwandler

\subsubsection{Ergebnisse PTV}

Die Reihenschaltung der Schallwandler hat sich als nicht praktikabel erwiesen, da die Schallwandler der Reihe nach eine abnehmende Amplitude haben, wie in Abbildung 4.12 an der Ausbreitung der Partikel zu erkennen ist.

Bei der Parallelschaltung zeigen alle Wandler eine ähnliche Amplitude, nur wird die elektrische Leistung auf die Wandler verteilt, was die maximale Amplitude stark limitiert. Im Weiteren wird daher, wie bei dem Experiment mit Tinte, immer ein Einzel-Wandler verwendet. 


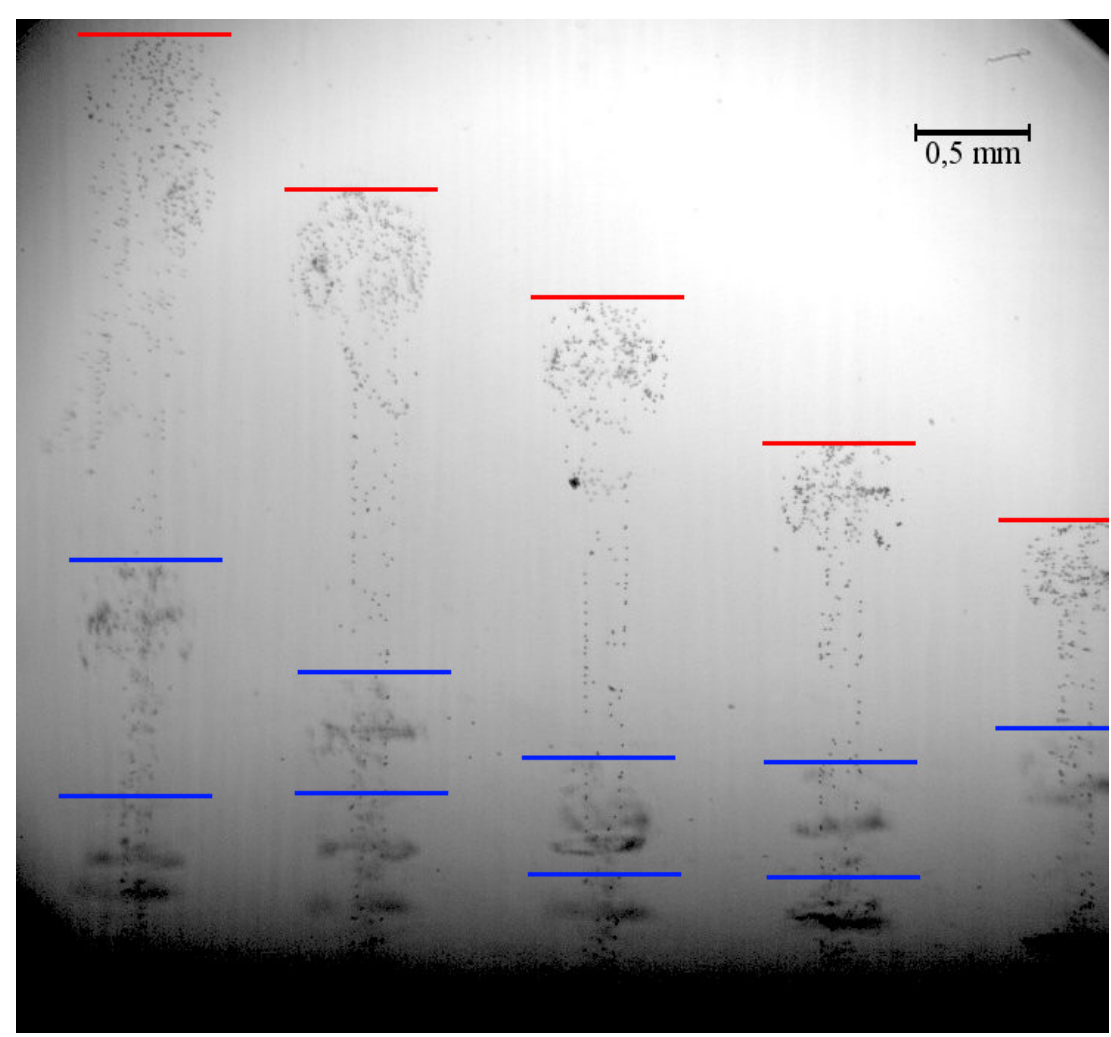

Abb. 4.12.: „Partikelpilze“, die durch eine Reihenschaltung von Schallwandlern erzeugt werden. Dabei sind die Wandler in 3 Reihen auf dem Chip verteilt, daher sind außerhalb des Fokus noch weitere Partikelpilze zu erkennen. Das obere Ende der „Pilze“ ist bei den „Pilzen" im Fokus durch einen roten Balken markiert, bei den Anderen durch einen blauen.

Das Particle Tracking wurde mit dem Plugin Mosaic [48] für ImageJ [49] ausgewertet. Hierzu wurden jeweils Sequenzen von 10000 Bildern einer Hochgeschwindigkeitsaufnahme ausgewertet. Da nicht nur die Partikel in der Ebene des Schallwandlers scharf abgebildet werden, sind in den Messwerten viele Partikel enthalten, die nicht die maximale Strömungsgeschwindigkeit haben. Ebenso werden auch Partikel neben der Jetströmung, die sich oberhalb des Wandlers befindet, mit verfolgt.

Exemplarisch zeigt Abbildung 4.13 die Geschwindigkeit aller Partikel, die durch das Tracking ermittelt werden konnten. Die Auflösung der Geschwindigkeit ist zum Einen durch die Bildrate der Kamera und zum Anderen die Größe der Bildpunkte limitiert. Bei den Messungen betrug die Bildrate 100,000 Bilder pro Sekunde und die Größe eines Pixels in der Aufnahme beträgt 3,17 $\mu \mathrm{m}$. Dadurch ergibt sich bereits durch die Verschiebung um einen Pixel pro Bild eine Geschwindigkeitsdifferenz von 0,317 m/s. Dies erklärt die Schichten in der Geschwindigkeitsverteilung in der Abbildung 4.13 . 

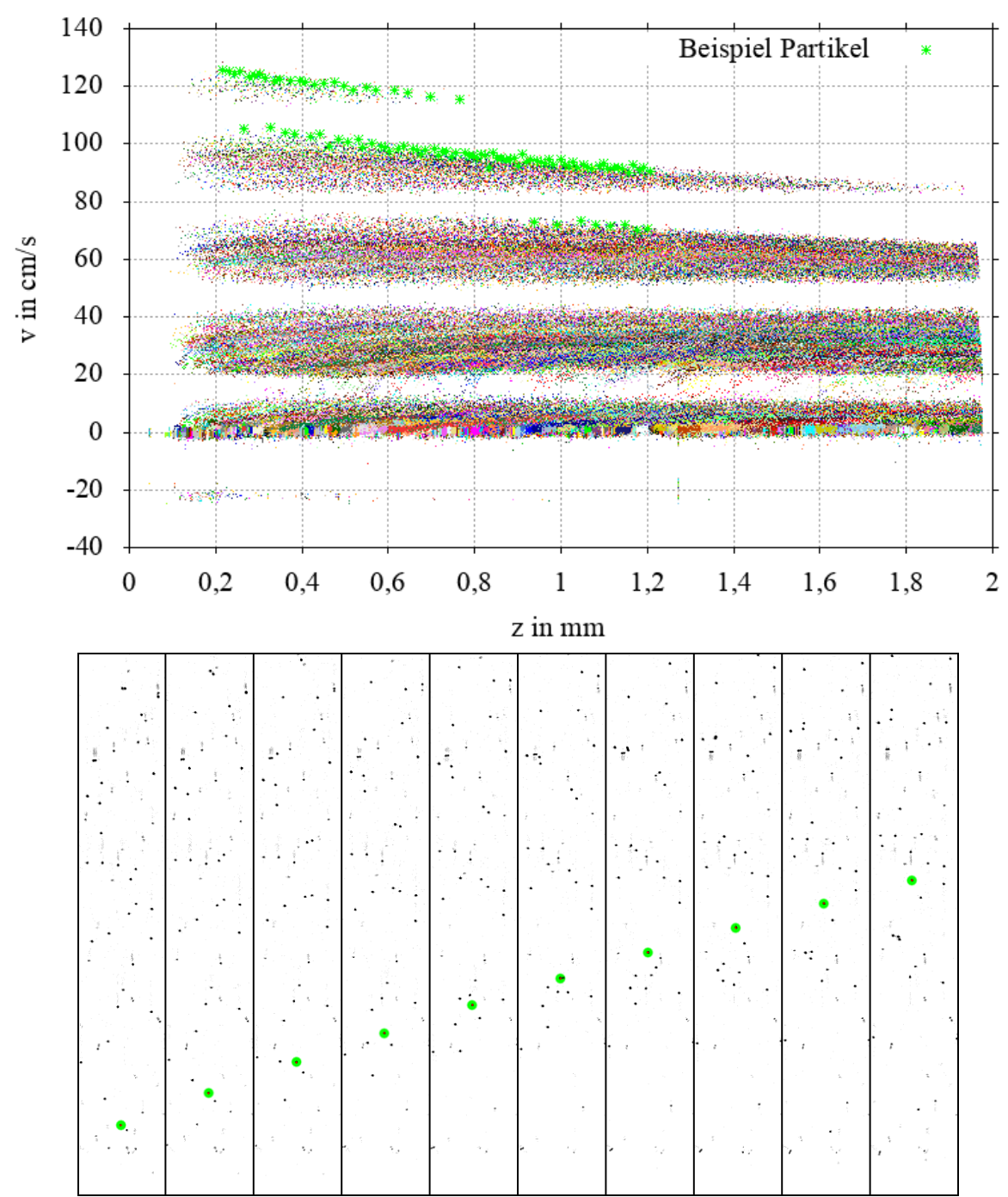

Abb. 4.13.: Alle Partikelgeschwindigkeiten aus einer Hochgeschwindigkeitsaufnahme, 10000 Bilder bei 100000 Bilder pro Sekunde. Der Schallwandler wurde mit $14,8 \mathrm{~V}_{\mathrm{pp}}$ gepulst betrieben bei einer Frequenz von $f=752,4 \mathrm{MHz}$. Exemplarisch wurde der Verlauf eines Partikels grün hervorgehoben und unten in der Bildserie markiert. Die Bildserie zeigt jedes 10te Bild einer Hochgeschwindigkeitsaufnahme. Ein der Montage hat eine Breite von 0,33 mm und eine Höhe von $2,03 \mathrm{~mm}$

Die Software versucht, die Geschwindigkeit noch durch Grauwerte der Partikel exakter zu bestimmen, was zu der Breite der Balken führt. 


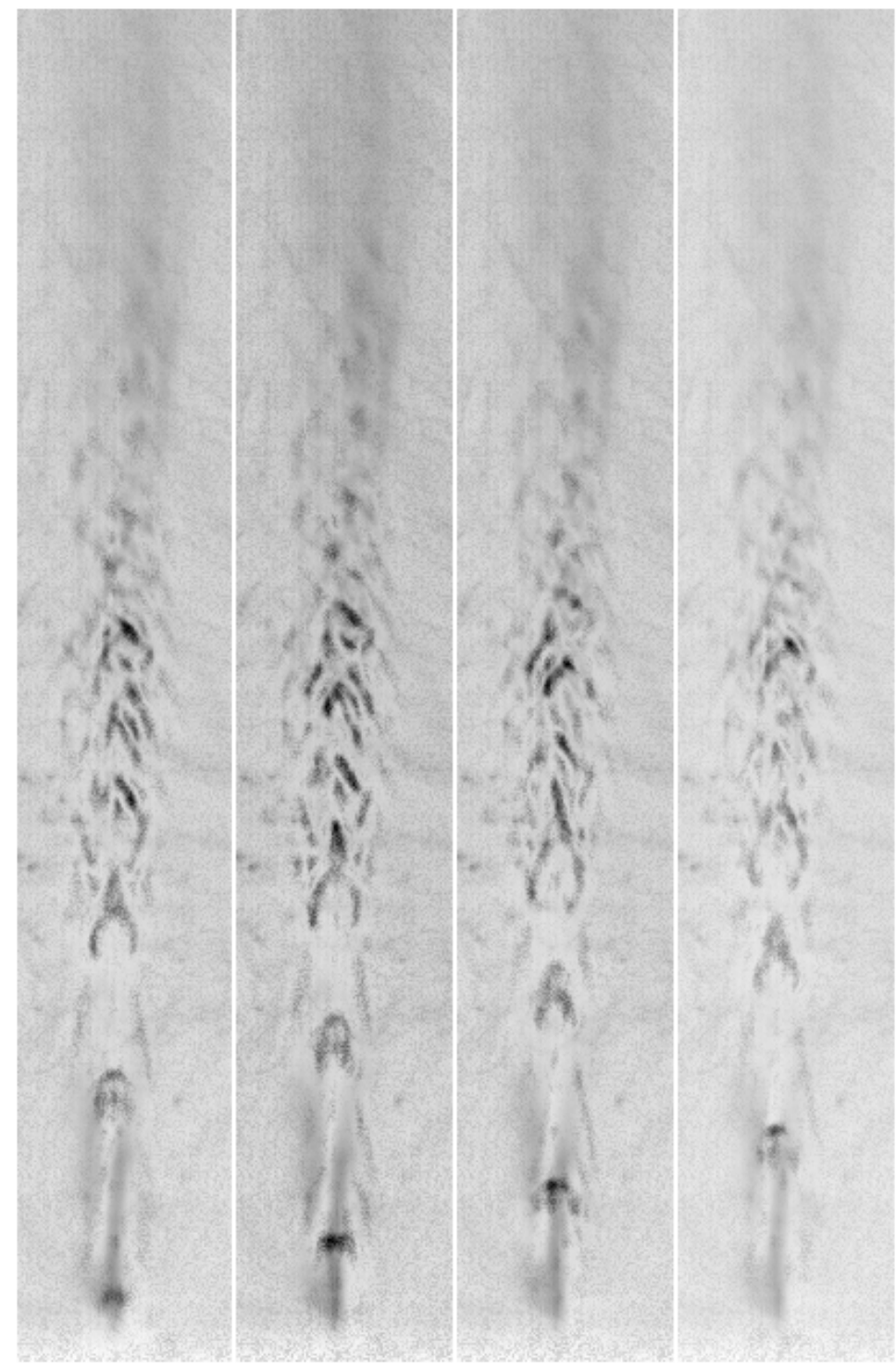

Abb. 4.14.: Temperaturschlieren bei einer Aufnahmegeschwindigkeit von 100000 Bildern pro Sekunde und einer Pulsrate von $25 \mathrm{kHz}$. Es ergeben sich Aufnahmen zu vier unterschiedlichen Phasenlagen. Dargestellt sind Mittelwerte aus 2500 Bildern je Phasenlage. Zusätzlich wurde der Mittelwert aus allen Aufnahmen abgezogen. Ein Bild der Montage hat eine Breite von 0,33 mm und eine Höhe von $2,03 \mathrm{~mm}$.

Bei höheren Amplituden werden Schlieren sichtbar, die auf einen Temperaturanstieg durch den Schallwandler schließen lassen. Diese Schlieren lassen sich beim Pulsbetrieb ebenfalls nutzen, um die Ausbreitung der Strömung zu visualisieren, wie es in Abbildung 4.14 gemacht wird. Die Schlieren können wie die Tinte im vorherigen Abschnitt zum Verfolgen der Strömung genutzt werden. Daraus lässt sich dann ebenfalls die Strömungsgeschwindigkeit ab- 


\section{Experimente}

schätzen.

Die oben gezeigten Experimente mit PTV wurden für unterschiedliche Spannungen und mit beiden Betriebsarten (gepulst und Dauerstrich) durchgeführt. Die maximalen Geschwindigkeiten der Experimente werden in Abbildung 4.15 zusammengefasst. Ebenfalls sind die durch Schlierenverfolgung ermittelten Geschwindigkeiten eingezeichnet.

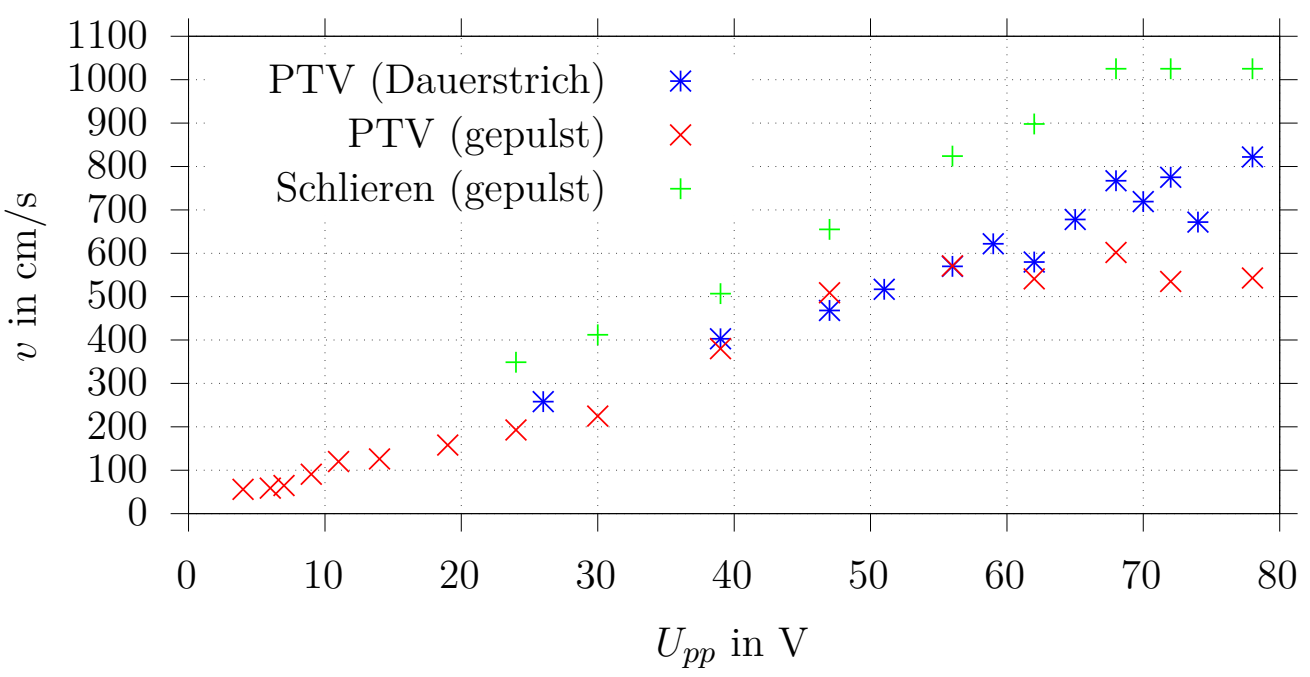

Abb. 4.15.: Geschwindigkeitsmessung aus Partikelverfolgung und Schlierenverfolgung

Die ermittelten Geschwindigkeiten geben den in Abschnitt 2.7.5 erwarteten linearen Verlauf der Strömungsgeschwindigkeit in Abhängigkeit von der Spannung wieder. Ebenfalls konnte kein großer Unterschied der maximalen Geschwindigkeit zwischen Puls und Dauerbetrieb festgestellt werden. Die Schlieren lassen vermuten, dass durchs Tracking die maximale Strömung nicht richtig aufgelöst wird, da zu wenige Partikel in die Bereiche mit der maximalen Strömungsgeschwindigkeit gelangen, wodurch die Strömung gerade bei größeren Amplituden unterschätzt wird.

Auch lassen sich bisher wenig Aussagen über das Geschwindigkeitsfeld sowie den zeitlichen Verlauf machen. Durch den PIV Aufbau aus dem folgenden Abschnitt wird es möglich das Strömungsfeld darzustellen.

\subsection{Particle Image Velocimetry (PIV)}

Wie beim PTV werden bei der Particle Image Velocimetry, kurz PIV, TracerPartikel in das Fluid gegeben. Jedoch werden bei der Auswertung nicht die Trajektorien der einzelnen Partikel verfolgt, sondern die Bewegung von Bildausschnitten durch Kreuzkorrelation ermittelt. Hierzu werden in der Regel 
zwei Bilder mit einem kurzen zeitlichen Abstand aufgenommen und mit einem Korrelationsalgorithmus verglichen.

\subsubsection{Versuchsaufbau PIV}

Folgende Skizze zeigt den optischen Teil des PIV Versuchsaufbaus. Die Ansteuerung des Schallwandlers findet wie bei dem Versuchsaufbau mit der Tinte (Abb. 4.8) statt.
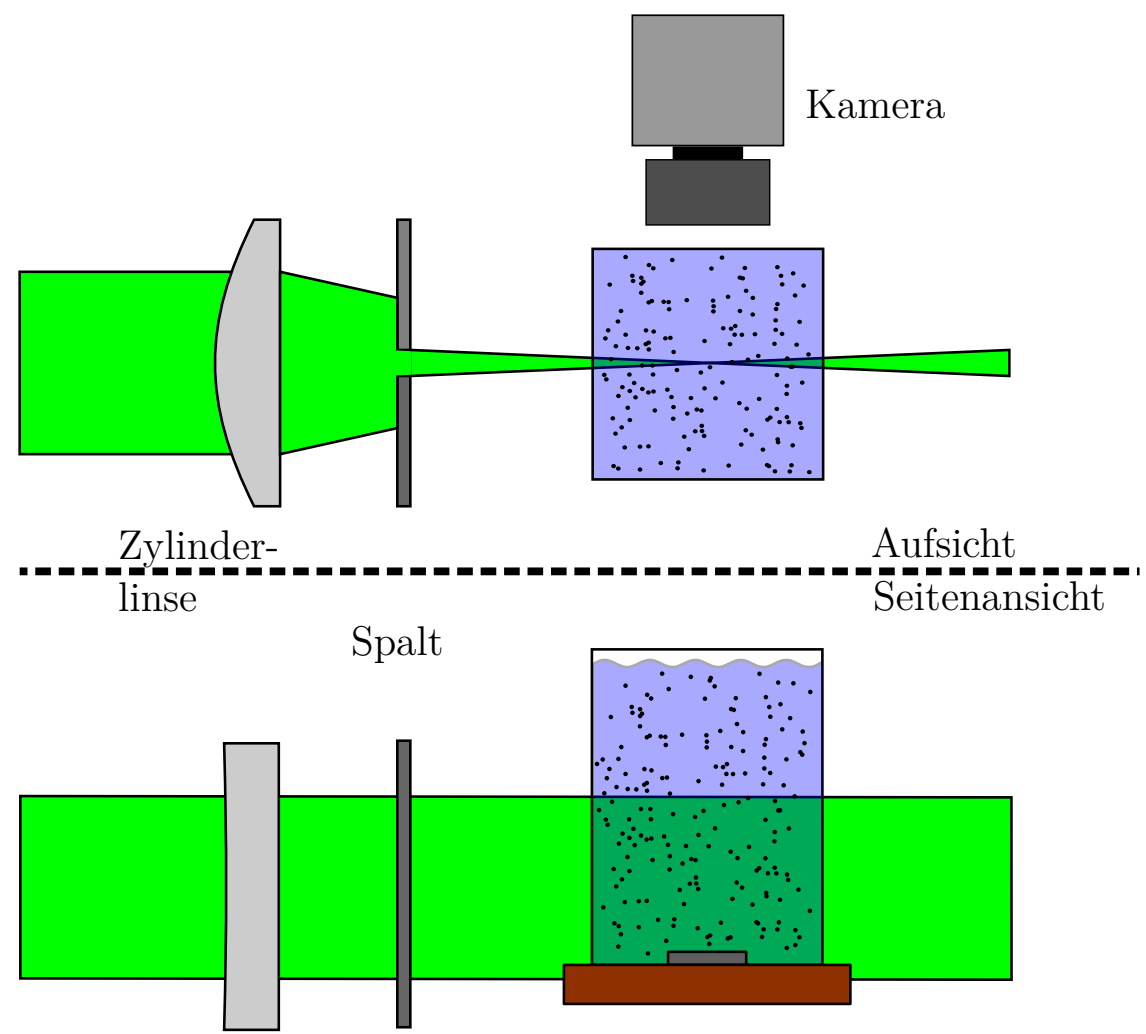

Abb. 4.16.: Skizze Versuchsaufbau PIV, grüner Laserlichtschnitt oberhalb des Schallwandlers

Als Erweiterung bzw. Ersatz der Geräte aus Tabelle 4.1 kommen folgende Gerätschaften zum Einsatz:

Tab. 4.2.: Zusätliche Geräte für den PIV-Aufbau

\begin{tabular}{ll}
\hline \hline Verstärker & Anfertigung von R.F.P.A. 200-800 MHz bis $100 \mathrm{~W}$ \\
PIV Laser & Litron NanoPIV LAS036 \\
Delaygenerator & Berkeley Nucleonics Corporation BNC 575 \\
Kamera & PCO Sensicam \\
Tracer Partikel & $\mathrm{SiO}_{2} 500 \mathrm{~nm}$ \\
Küvette & Helma Quarzglas
\end{tabular}




\section{Experimente}

Neben den Geräten für den Laser-Lichtschnitt wird ein Delaygenerator für die Synchronisation der Geräte verwendet. Der Verstärker wird durch einen leistungsfähigeren ersetzt, was höhere Spannungen an den Wandlern ermöglicht. Eine Küvette aus Quarzglas ist auch von den optischen Eigenschaften her besser geeignet als die vorher verwendete PMMA Küvette.

Eine PIV-Aufnahem besteht aus 2 Kamerabildern die jeweils mit einem Laserpuls beleuchtet werden. Die verwendete Kamera ist mit einer Aufnahmegeschwindigkeit von $5 \mathrm{~Hz}$ je Doppelbild der limitierende Faktor bei der Wiederholrate. Dafür beträgt die minimale Zeit zwischen den Doppelbildern nur 500 ns. Der Schallwandler wird synchronisiert mit der Aufnahme betrieben, also für jede Aufnahme wird der Schallwandler bis zu $2 \mathrm{~ms}$ im Dauer- bzw. Pulsbetrieb geschaltet. Dadurch können beliebige Phasenlagen der Pulse reproduzierbar aufgezeichnet werden, was eine Mittlung über viele Pulse ermöglicht. Die Auswertung der PIV Aufnahmen erfolgt mit PIVLAB [50] für Matlab.

Zusätzlich kann noch eine Grenzfläche in die Strömung vor dem Wandler angebracht werden, um das Strömungsverhalten der akustisch getriebenen Strömung in der Nähe einer Grenzfläche zu messen. Hierzu wird ein kleines Stück Siliziumwafer, welches an eine Halterung geklebt ist, in die Küvette getaucht.

\subsubsection{Ergebnisse PIV}

Durch die PIV Aufnahmen lässt sich das durch die akustisch generierte Volumenkraft resultierende Strömungsfeld darstellen und somit auch die maximale Strömungsgeschwindigkeit in Abhängigkeit der Spannung am Schallwandler ermitteln, wie in Abbildung 4.17 dargestellt.

Der große Vorteil von PIV ist, dass nicht nur einzelne Geschwindigkeiten bestimmt werden, sondern eine ganze Ebene gemessen wird, wie die Abbildung 4.18 zeigt, in der das Strömungsfeld zu unterschiedlichen Zeiten nach Anlegen der Spannung an den Schallwandler dargestellt ist. 


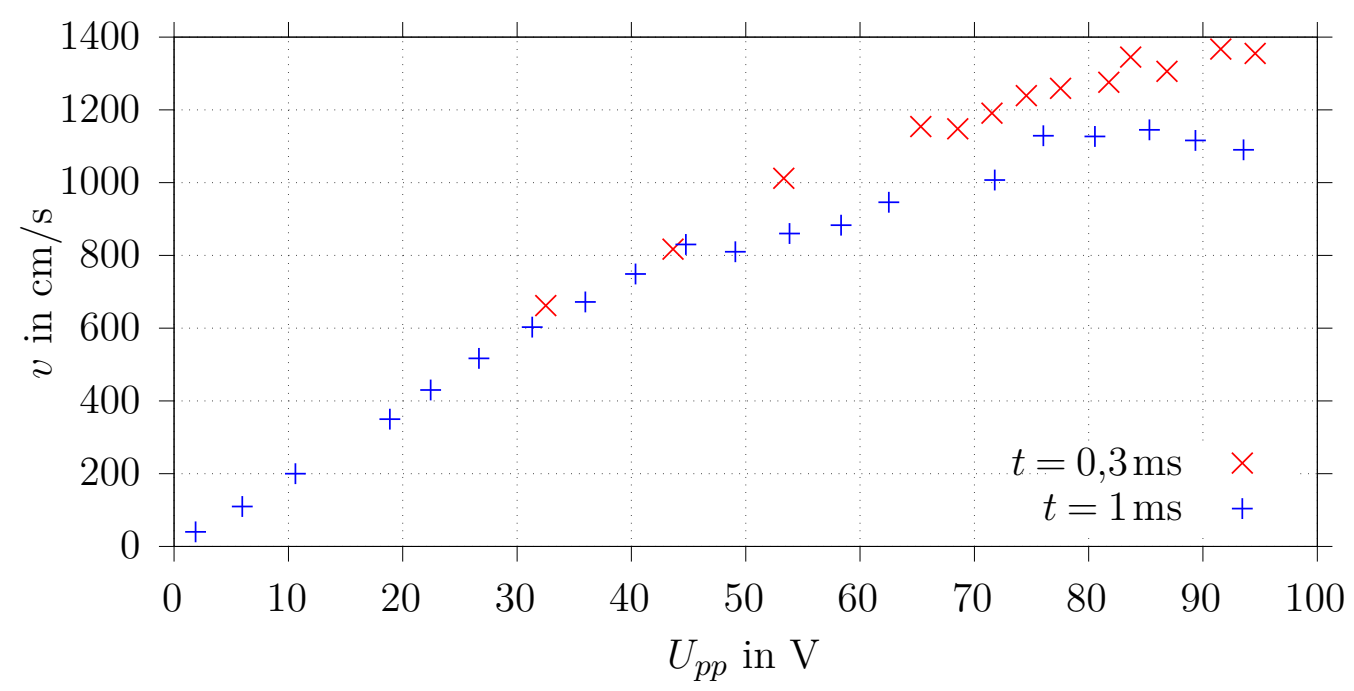

Abb. 4.17.: Maximale Strömungseschwindigkeiten der PIV-Messungen in Abhängigkeit von der Spitze-Spitze Spannung am Schallwandler zum Zeitpunkt 0,3 ms und $1 \mathrm{~ms}$ nach anlegen der Spannung. Die Betriebsdauer ist jeweils $0,1 \mathrm{~ms}$ länger.

Da eine einzelne PIV Aufnahme lückenhaft bzw. mit Fehlern versehen ist, wird bei den dargestellten Bildern immer der Mittelwert aus den Resultaten von 100 Doppelaufnahmen gebildet.

Bei den ersten Bildern in Abb. 4.18 ist noch die Ausbreitung des Strömungsfelds mit dem pilzförmigen Anfahrtswirbel am oberen Ende zu erahnen. Da die Strömungsgeschwindigkeiten abseits des Zentrums aber deutlich langsamer sind als im Zentrum, wird der Wirbel nicht gut aufgelöst. Mit längerer Laufzeit des Wandlers lassen die Verwirbelungen um die jetförmige Strömung nach und das Strömungsprofil ähnelt zunehmend dem eines Freistrahls. Auch nimmt die Strömungsgeschwindigkeit über die Dauer der Messung ab, was vermutlich an der Erwärmung des Wandlers und einer Resonanzverschiebung liegt.

Das Strömungsfeld stimmt mit der Erwartung aus der numerischen Simulation aus Abschnitt 3.5 gut überein. Die Beschleunigung des Fluides durch die Volumenkraft des Schallfeldes findet demnach innerhalb von $\approx 100 \mu \mathrm{m}$ vor dem Wandler statt und im weiteren Verlauf ist nur die Massenträgheit für die Strömung verantwortlich. Nach dem transienten Anfahrtsvorgang bildet sich ein recht scharf begrenzter, wirbelfreier Strahl aus, der einem laminaren Freistrahl gleichkommt. 


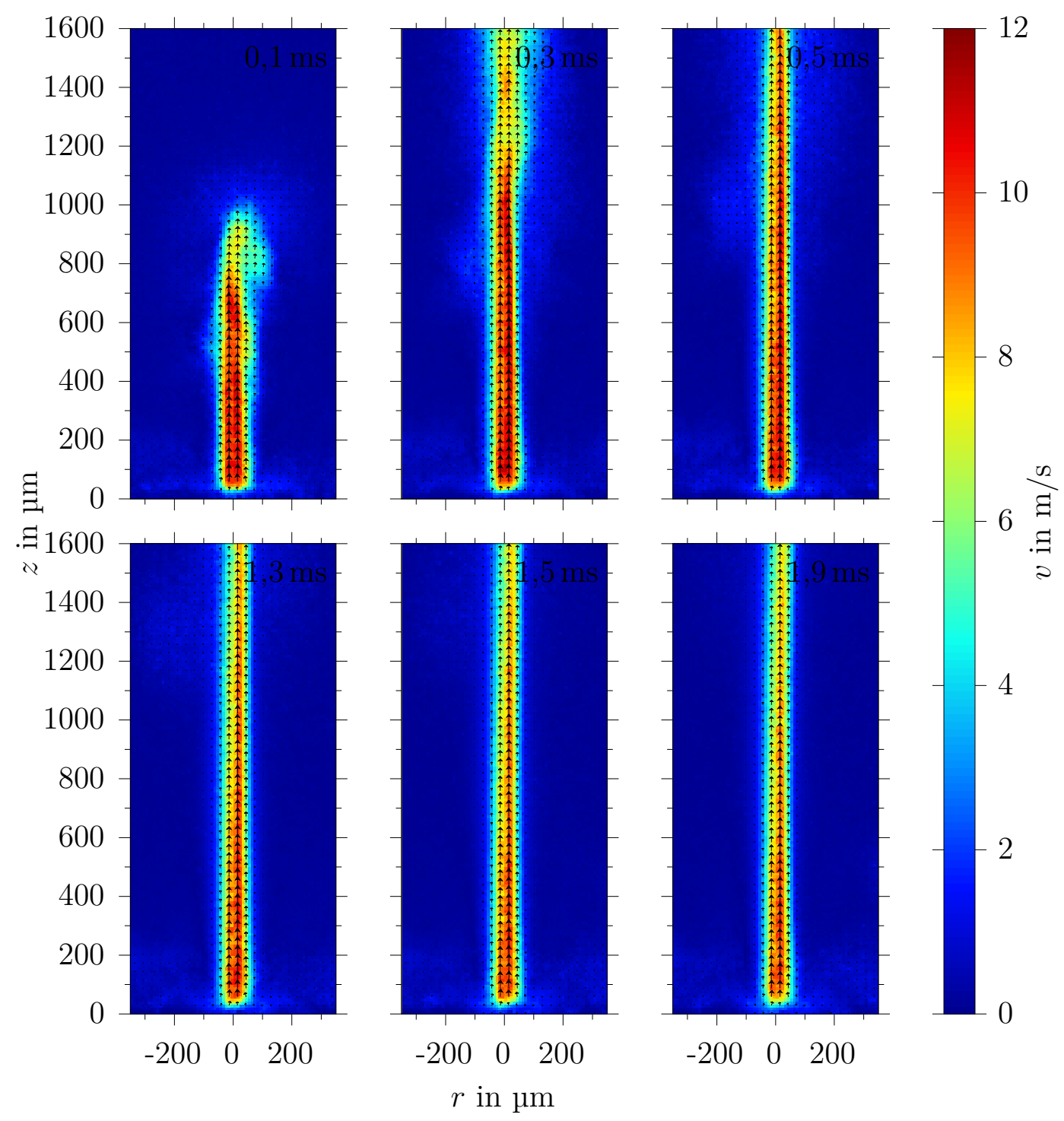

Abb. 4.18.: Schallwandler wurde mit 760,7 MHz betrieben und einer Spannung von $U_{p p} \approx 83,7 \mathrm{~V}$. Der Zeitpunkt der Aufnahme nach Anlegen der Spannung ist oben rechts angegeben.

\subsubsection{Pulsbetrieb}

Der Schallwandler kann auch ohne Probleme im schnellen Pulsbetrieb arbeiten, wie bereits im Abschnitt 4.3.2 verwendet. Hierdurch werden aufeinanderfolgende Anfahrtswirbel erzeugt, die auch in den PIV Aufnahmen zu erkennen sind. 


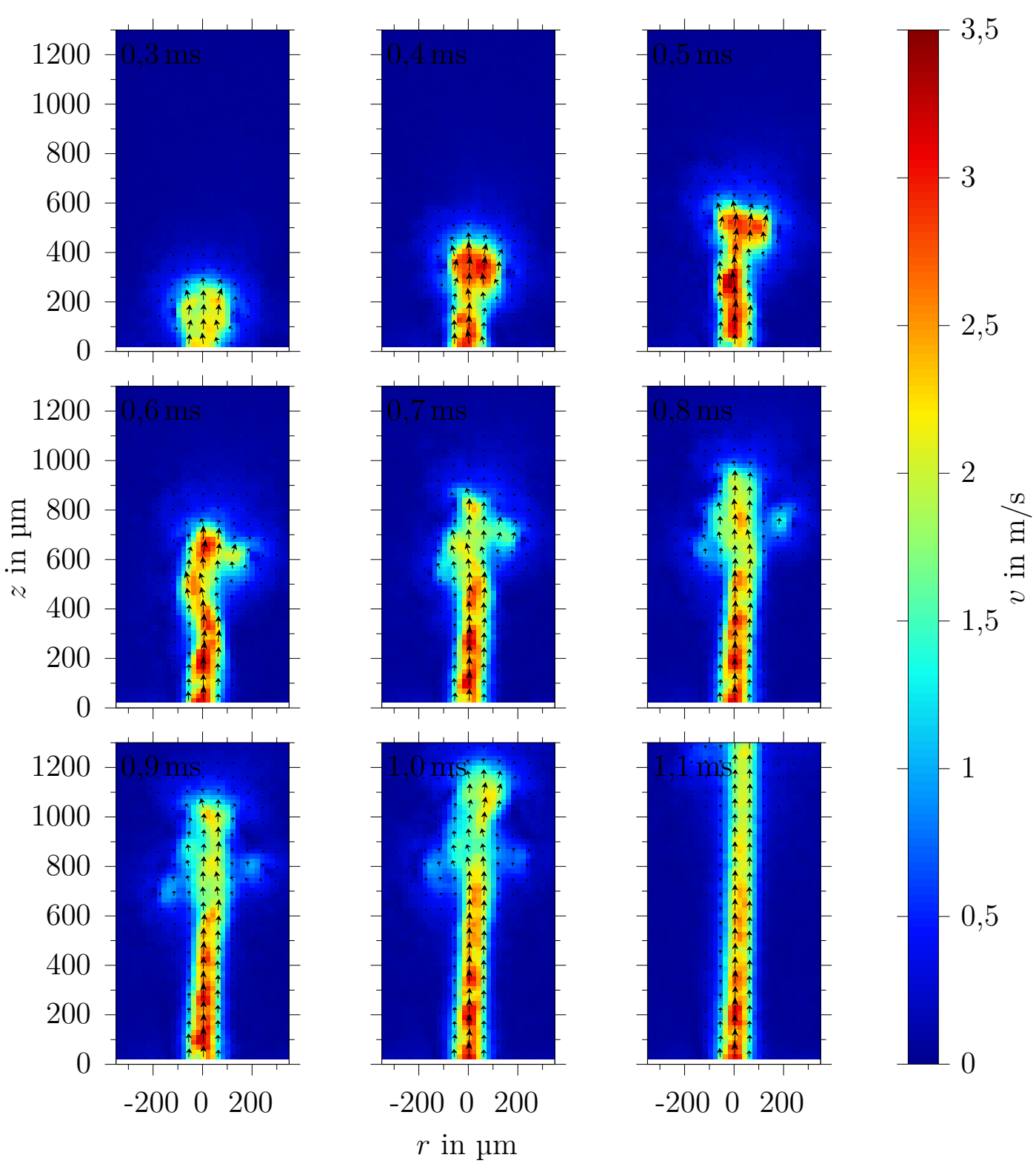

Abb. 4.19.: PIV-Aufnahmen im schnell gepulsten Betrieb. Der Schallwandler wird mit 797,4 MHz und einer Spannung von $U_{p p} \approx 33 \mathrm{~V}$ betrieben. Das erst Bild zeigt die Strömung 0,3 ms nach Anlegen der Spannung. Die weiteren Bilder sind immer 0,1 ms später aufgenommen. Die Zeiten sind links oben in den Bildern angegeben.

Beim Pulsbetrieb wird wie beim PTV aus Abschnitt 4.3 eine Frequenz von $25 \mathrm{kHz}$ gewählt mit einer Anzeit von $50 \%$. Da der Schallwandler eine Arbeitsfrequenz von $\approx 750 \mathrm{MHz}$ hat, beinhaltet ein einzelner Puls noch immer 15000 Schallfeldperioden. Aus dem Abstand der Wirbel lässt sich auch eine Ausbreitungsgeschwindigkeit abschätzen. Diese liegt mit $v \approx 4 \mathrm{~m} / \mathrm{s}$ im Bereich der durchs PIV ermittelten Geschwindigkeit im Zentrum der Wirbel. Es ist auch 


\section{Experimente}

zu erahnen, dass die ersten Pulse sich langsamer ausbreiten als die folgenden, die im bereits beschleunigten Fluid erzeugt werden.

\subsubsection{Betrieb mit kurzen Einzelpulsen}

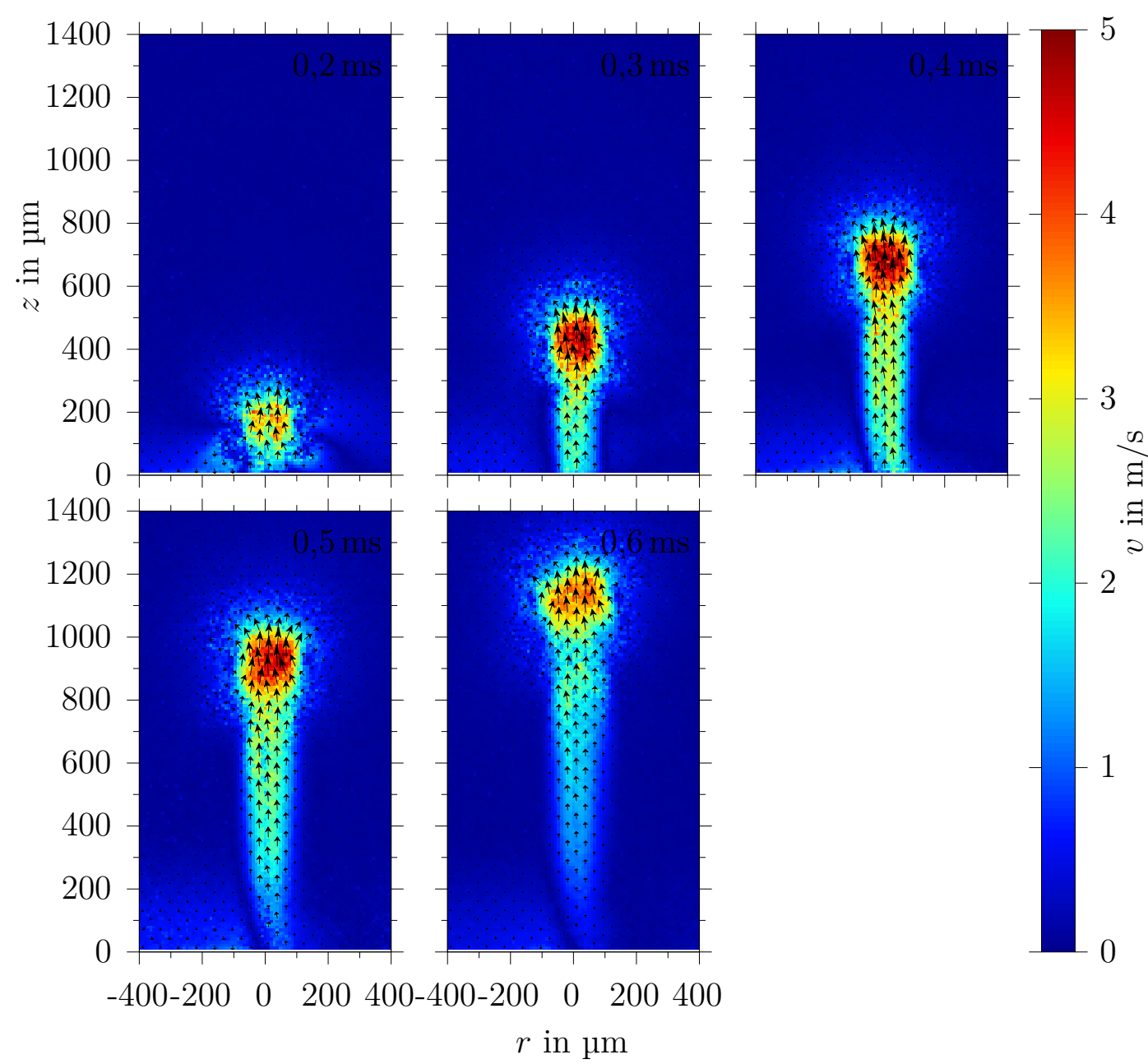

Abb. 4.20.: PIV-Aufnahme bei einem kurzem Einzelpuls. Der Schallwandler wurde mit $f=632,9 \mathrm{MHz}$ bei einer Spannung von $U_{p p} \approx 30 \mathrm{~V}$ für 0,35 ms betrieben. Der Aufnahmezeitpunkt nach Anlegen der Spannung ist rechts oben in den Bildern angegeben.

Für die Experimente der kurzen Einzelpulse wird ein Schallwandler der dritten Generation verwendet, der verglichen mit den anderen Generationen eine höhere Strömungsgeschwindigkeit pro angelegter Spannung aufweist aber durch die dünne Siliziummembran auch anfälliger für Defekte ist.

Abbildung 4.20 zeigt die Strömung eines 350 us langen Pulses mit einer Anregungsfrequenz von $f=632,9 \mathrm{MHz}$. Ein Anfahrtswirbel wie der Kopf bei den 
„Tintenpilzen“ aus Abschnitt 4.2 wird erzeugt und bewegt sich durchs Wasser. Die bei längeren Pulsen erzeugte Jetströmung hinter dem Wirbel ist bei dem kurzen Puls kaum vorhanden und die Strömung fällt schnell wieder ab.

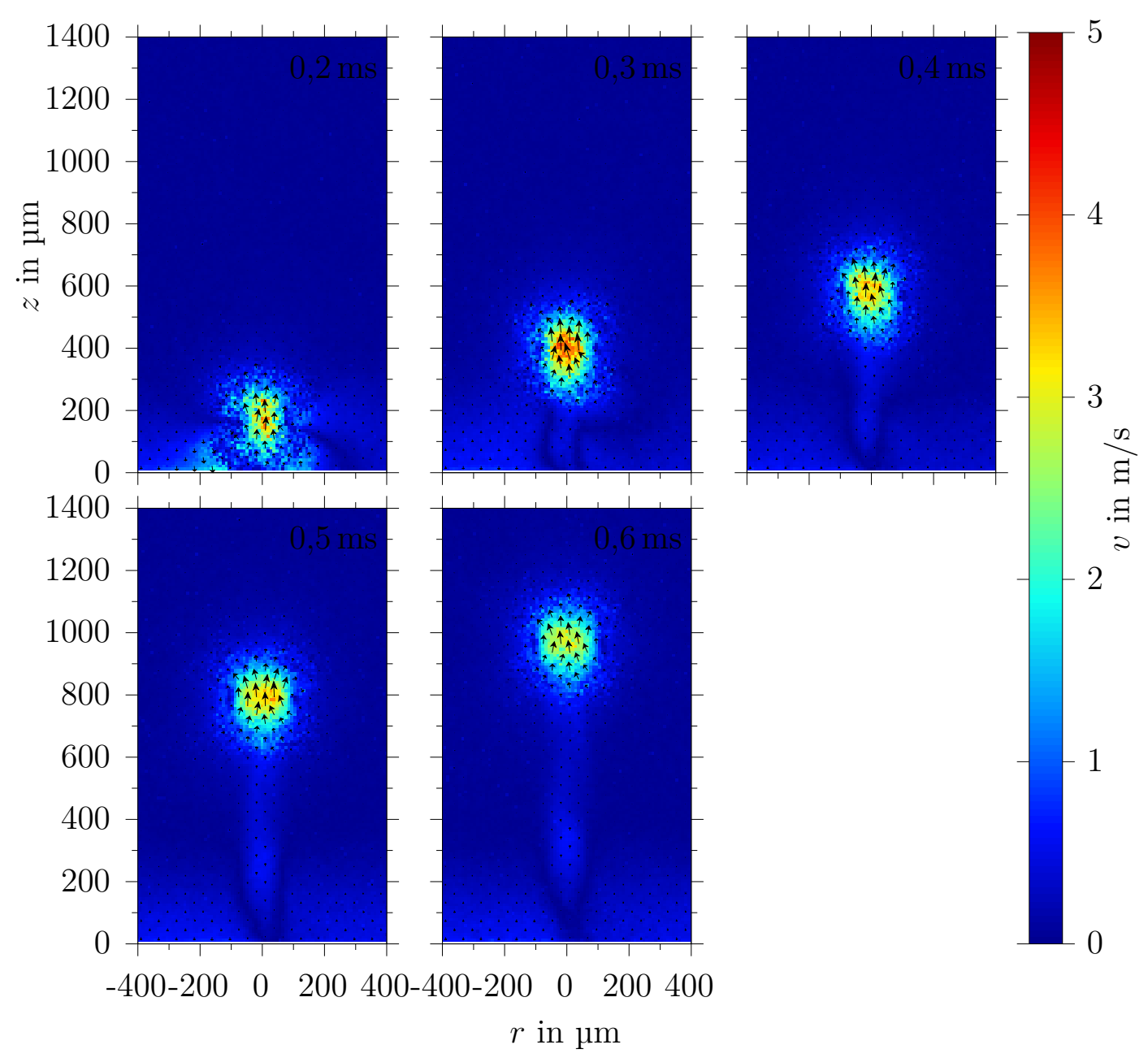

Abb. 4.21.: PIV-Aufnahme bei einem sehr kurzen Einzelpuls. Der Schallwandler wurde mit $f=632,9 \mathrm{MHz}$ bei einer Spannung von $U_{p p} \approx 30 \mathrm{~V}$ für $0,1 \mathrm{~ms}$ betrieben. Der Aufnahmezeitpunkt nach Anlegen der Spannung ist rechts oben in den Bildern angegeben.

Bei einem noch kürzeren Puls von $100 \mu$ s wie in Abbildung 4.21 ist der Effekt der geringen Strömung nach dem Wirbel noch ausgeprägter und die nachströmende Flüssigkeit ist kaum noch messbar. Jedoch reduziert sich beim kürzeren Puls die maximal erreichte Strömungsgeschwindigkeit bei gleicher Anregung. Die Geschwindigkeit, mit der sich die Wirbel durch die Flüssigkeit bewegen, beträgt $u_{a} \approx 2,6 \mathrm{~m} / \mathrm{s}$ bei dem $350 \mu \mathrm{s}-\mathrm{Puls}$ und $u_{a} \approx 2 \mathrm{~m} / \mathrm{s}$ bei dem $100 \mu \mathrm{s}-P u l s$. Sie ist damit deutlich langsamer als die Geschwindigkeit im Wirbel. 


\subsubsection{Strömung an einer festen Grenzfläche}

In diesem Abschnitt wird die entstehende Strömung, die sich beim Auftreffen des akustisch generierten Strahls auf eine feste Grenzfläche ausbildet, gemessen. Auf Grund der Beleuchtung von der Seite ist die Grenzfläche nicht exakt parallel zum Schallwandler ausgerichtet, sondern in einem leichten Winkel, wie am oberen Rand auf der linken Hälfte in Abbildung 4.22 zu sehen.

Auf der rechten Seite von Abbildung 4.22 wird eine nach Abschnitt 3.5 .2 simulierte Strömung gezeigt, bei der eine Grenzfläche parallel zum Schallwandler in einem Abstand von 1,6 mm angenommen wird. Für die Berechnung der Volumenkraft nach Abschnitt 2.7.4 wird eine Schallschnelle von $u_{0}=25 \mathrm{~m} / \mathrm{s}$ angesetzt.

Qualitativ ist das Ergebnis der Messung, die auf der linken Seite gezeigt ist, und der Simulation recht ähnlich. Eine jetförmige eng begrenzte Strömung reicht vom Schallwandler bis zur Grenzfläche, an der ein Staupunkt im Zentrum der Strömung zu sehen ist. Der Anfahrtswirbel, der auf die Grenzfläche trifft, bewegt sich seitlich nach außen.

Quantitativ sind Unterschiede in der Strömungsgeschwindigkeit zu erkennen, und auch die Breite des Jets ist bei der Messung größer als in der Simulation. Auch ist gerade im oberen Bereich noch eine erhöhte Geschwindigkeit in der Flüssigkeit neben dem Jet zu sehen die so nicht in der Simulation vorhanden ist. In der Simulation wird die Strömung als laminar angenommen.

Mit einem $R e_{\text {hy }} \approx 2400\left(\rho \approx 1000 \mathrm{~kg} / \mathrm{m}^{3}, v \approx 12 \mathrm{~m} / \mathrm{s}, d \approx 200 \mu \mathrm{m}\right.$ und $\mu \approx$ 0,001 Pas) ist das Einsetzen von Turbulenzen zu erwarten, was eine mögliche Erklärung für die Unterschiede ist.

Die unterschiedlich weite Ausbreitung des Wirbels an der Oberfläche liegt möglicherweise auch daran, dass bei der PIV Messung die Geschwindigkeit der Strömung unterschätzt wird und daher sich die Zeitskala vom Auftreffen des Wirbels und die Ausbreitung über die Oberfläche zwischen Messung und Simulation unterscheidet. 


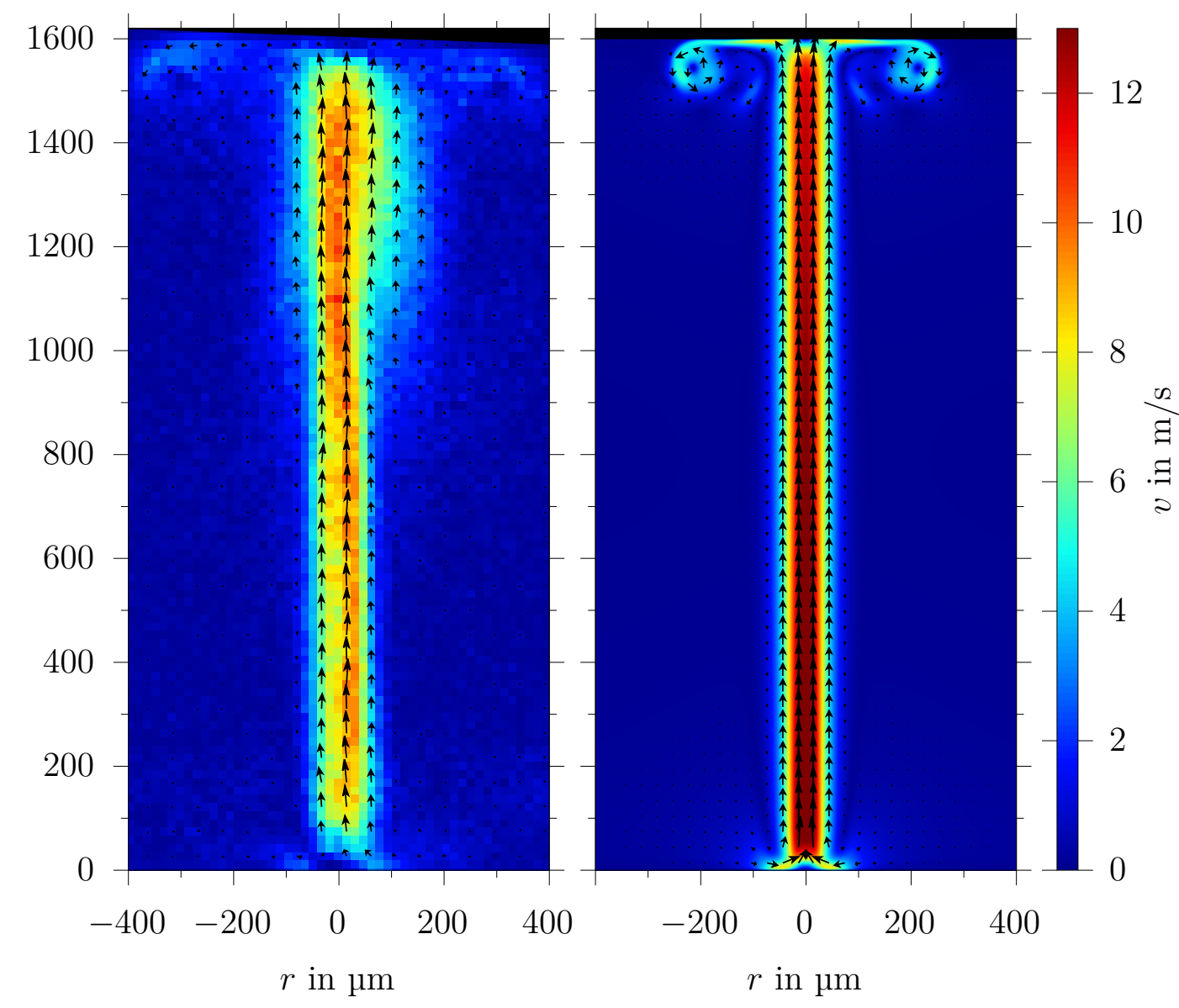

Abb. 4.22.: Links: PIV-Aufnahme des Experiments, bei dem der Schallwandler mit $760,7 \mathrm{MHz}$ und einer Spannung von $U_{p p} \approx 83,7 \mathrm{~V}$ betrieben wird, 0,3 ms nach Anlegen der Spannung gemessen. Rechts: Nichtlineare Simulation mit Grenzfläche. 


\subsection{Chronoamperometrie}

Mit einem Versuchsaufbau, der dem von Reuter in [51] vorgestellten vergleichbar ist, wurde die Strömung untersucht. Als Flüssigkeit wird hier eine wässrige Elektrolytlösung verwendet. Durch eine Mikroelektrode, die in einen Glasstab eingelassen ist, kann durch das chronoamperometrische Messverfahren die Störung der elektrodennahen Flüssigkeitsströmung als elektrischer Strom gemessen werden. Durch Anlegen einer Spannung zwischen der Mikroelektrode und einer Referenzelektrode wird ein Ladungsfluss erzeugt, der durch die oxidierbaren Ionen an der Mikroelektrode limitiert wird. Einige ms nach Anlegen der Spannung sind alle Ladungsträger an der Mikroelektrode verbraucht, und nur durch Diffusion von neuen Ladungsträgern zur Elektrode wird ein geringer Strom aufrecht erhalten. In diesem Zustand reagiert der Strom durch die Mikroelektrode empfindlich und mit hoher zeitlicher Aufösung auf Fluidströmungen, die nichtoxidierte Ladungsträger an die Elektrode bringen. [52, 53, 54 ]

\subsubsection{Versuchsaufbau Chronoamperometrie}

Die verwendete Elektrode besteht aus einem Platindraht, der an der Spitze einen Durchmesser von $16 \mu \mathrm{m}$ hat. Dieser Draht befindet sich in einem Glasstab mit einem Durchmesser von $4 \mathrm{~mm}$.

Mit der Elektrode werden horizontale Ebenen parallel zur Wandleroberfläche von $10 \times 10$ Punkten abgefahren. Der Abstand zwischen zwei benachbarten Punkten beträgt sowohl in $x$ - als auch $y$-Richtung $25 \mu \mathrm{m}$. Es werden sieben Ebenen in unterschiedlicher Höhe $z$ von $110 \mu \mathrm{m}$ bis $2370 \mu \mathrm{m}$ über dem Schallwandler gemessen.

Für jeden der 100 Messpunkte je Ebene wird der Schallwandler für $1 \mathrm{~ms}$ betrieben.

Die Geräte für den Betrieb der Schallwandler sind identisch mit dem vorherigen Versuchsaufbau der PIV in Abschnitt 4.4.1. Zur Messung des elektrischen Stroms wird ein Potentiostat des Typs Gamry Reference 600 verwendet. Als Elektrolyt dient Ruthenium Hexamin Chlorid mit der Formeleinheit $\left[\mathrm{Ru}\left(\mathrm{NH}_{3}\right)_{6}\right] \mathrm{Cl}_{3}$ mit Kaliumnitrat $\mathrm{KNO}_{3}$ als Pufferlösung [55], [56].

\subsubsection{Ergebnisse}

Der elektrischen Strom, der durch die Elektrode fließt, ist im gemessenen Bereich proportional zur Flüssigkeitsströmung an der Elektrode. Ohne Strömung stellt sich bei konstanter Spannung ein durch Diffusion begrenzter elektrischer Strom ein. Wenn durch eine Strömung neue Ionen an die Elektrode gebracht werden, ist das durch einen Anstieg im elektrischen Strom zu erkennen.

Abbildung 4.23 zeigt die beiden Messebenen, die in einer Höhe von $110 \mu \mathrm{m}$ 
und $230 \mu \mathrm{m}$ übder dem Wandler aufgenommen wurden. Zum Zeitpunkt $t=0 \mathrm{~s}$ wird die Spannung am Schallwandler angelegt.

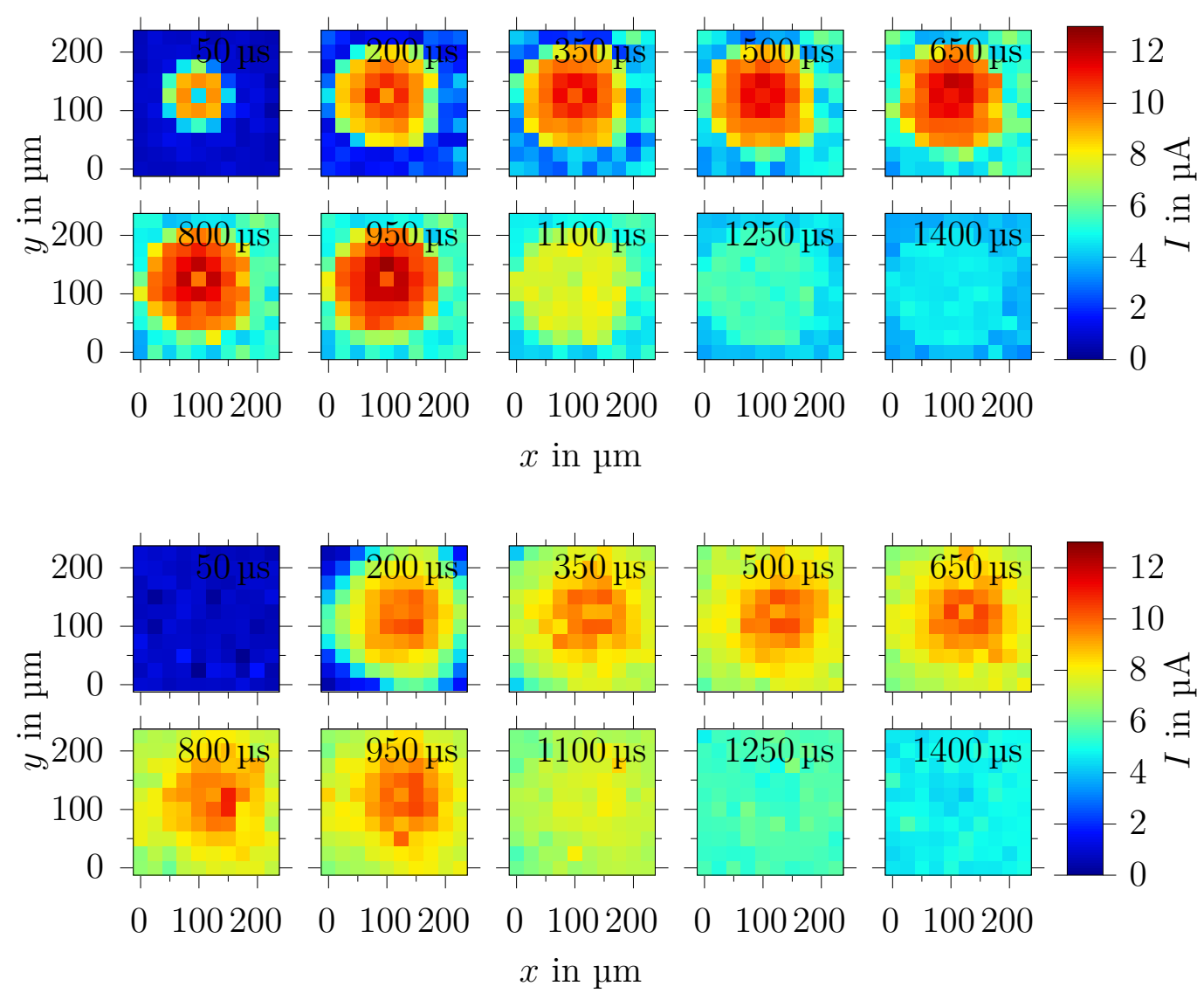

Abb. 4.23.: Gemessener Strom zu unterschiedlichen Zeitpunkten $t$ rechts oben in den Bildern dargestellt, für $z=110 \mu \mathrm{m}$ (oben) und $z=230 \mu \mathrm{m}$ (unten).

Der maximale gemessene Strom einer Ebenen ist zeitaufgelöst für die sieben gemessenen Ebenen in der Abbildung 4.24 dargestellt. Bereits nach $\approx 30 \mu \mathrm{s}$ ist bei der Messebene mit einem Abstand von $110 \mu \mathrm{m}$ ein deutlicher Anstieg im Stromfluss zu erkennen. Im Zentrum der Messebenen ist ein Staupunkt zu erkennen, an dem der gemessene Strom kleiner als in der Umgebung ist. Auch ist der Bereich, in dem ein Anstieg des Stroms zu erkennen ist, in der Größenordnung des Durchmessers des Schallwandlers von $\approx 200 \mu \mathrm{m}$. 


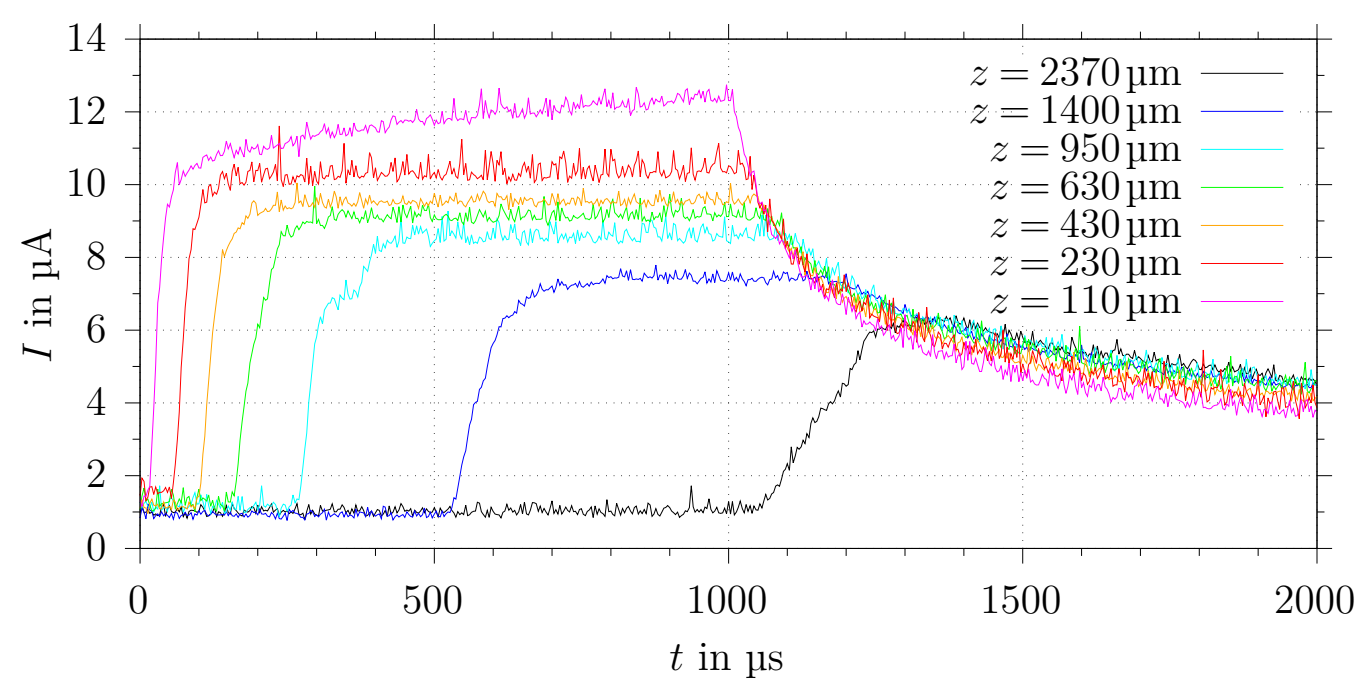

Abb. 4.24.: Maximal gemessener Elektrodenstrom der 100 Messpunkte gegen die Zeit nach Einschalten des Schallwandlers für $1 \mathrm{~ms}$.

Mit größer werdendem Abstand vom Schallwandler und mit der Zeit wird auch der Bereich, in dem eine Strömung gemessen wird, größer. Mit dem Ausschalten des Wandlers fällt die Strömung mit einer Verzögerung durch die Laufzeit der Strömung etwa exponentiell ab. Ebenso nimmt der maximal gemessene Strom mit dem Abstand zum Wandler ab, was auch durch die schwächer werdende Flüssigkeitsströmung zu erwarten ist.

Bei der Messebene im Abstand von $110 \mu \mathrm{m}$ kommt es über die Laufzeit des Wandlers zu einem weiteren Anstieg des maximalem Stroms, während sich bei den anderen Ebenen ein konstanter Wert einstellt. Man kann annehmen, dass der weitere Anstieg durch die steigende Temperatur in dem kleinen Flüssigkeitsvolumen zwischen Wandler und Elektrode erzeugt wird.

Mit den Messungen erkennt man, dass durch die akustisch erzeugte Strömung diffusionsbegrenzte Reaktionen an Oberflächen beschleunigt werden. Ebenso entstehen Scherkräfte, die mit entsprechenden Modellen auch quantifiziert werden könnten [51]. Hier wird lediglich qualitativ festgestellt, dass die akustische Strömung auf sehr kleinen Skalen „berührungslos“, schnell und gut steuerbare Agitation von Fluiden an festen Oberflächen bewirken kann. Anwendungen hiervon liegen z.B. in der Oberflächenchemie oder der Reinigung. 


\subsection{Experimente mit Luminol}

Bei der Visualisierung von Kavitation in Ultraschallbädern kann Luminol eingesetzt werden: Falls sich beim Kavitationsblasenkollaps Radikale bilden wird das Luminol zur Lumineszenz angeregt. In Bereichen mit hoher Schallintensität sind durch die höheren Drücke die Blasenkollpase intensiver und damit auch die Lumineszenz [57]. Dadurch wird dann auch das Schallfeld sichtbar gemacht.

\subsubsection{Ergebnisse Luminol}

Bei den verwendeten hochfrequenten Schallwandlern konnte keine Lumineszenz gemessen werden. Trotz Tests mit argongesättigter Luminollösung, die im kavitierenden Ultraschallbad besonders intensiv leuchtet, konnte mit einer Nikon D700 mit einem 50 mm Objektiv mit maximal geöffneter Blende auch bei einer Belichtungszeit von $30 \mathrm{~s}$ keine Emission gemessen werden. Mit vermuteten Schalldrücken von bis zu $30000 \mathrm{kPa}$ am Schallwandler ist zwar nach den Berechnungen aus Abschnitt 2.8 mit dem Einsetzen von Kavitation zu rechnen. Das negative Messergebnis lässt allerdings vermuten, dass entweder keine entsprechend kleinen Kavitationskeime vorhanden waren bzw. der Blasenkollaps bei der Größe zu sanft ist, um Radikale für die Anregung des Luminols zu erzeugen.

Es bleibt daher weiter zu klären, ob bei diesen Schallfrequenzen überhaupt Kavitation erzeugt werden kann. 
4. Experimente 


\section{Fazit}

Auch wenn akustische Strömungen schon vor langer Zeit entdeckt und theoretisch beschrieben wurden, ist der UHF-Bereich noch recht wenig untersucht. Durch die hohen Frequenzen und Amplituden war das Einbeziehen von nichtlinearer Akustik zur Beschreibung des Phänomens nötig.

Die neuartigen Schallwandler haben es ermöglicht, besonders hohe Strömungsgeschwindigkeiten in kleinen Raumskalen zu erzeugen und präzise zu kontrollieren. Da nicht alle Parameter durch die kleinen zeitlichen und räumlichen Skalen einer direkten Messung zugänglich sind, wird durch die vorgestellten Simulationen das Bild vervollständigt. Zudem sind die Experimente auf die durch den Schallwandler festgelegte Frequenz beschränkt. Auch hier ermöglichen die Simulationen einen vollständigeren Blick auf das Phänomen.

Gerade das Schallfeld mit einer Frequenz von $\approx 750 \mathrm{MHz}$ und damit einer Wellenlänge von $\lambda=2 \mu \mathrm{m}$ ist für eine Messung nur schwer zugänglich, stellt aber die Grundlage für die Beschreibung der Strömung dar. Mit einem Durchmesser von $\approx 100 \mu \mathrm{m}$ ist die Strömung um fast zwei Größenordnungen größer, aber fällt immer noch in den Bereich der Mikrofluidik. Die gemessen Strömungsgeschwindigkeiten von über $12 \mathrm{~m} / \mathrm{s}$ sind für diese Größenordnung bemerkenswert.

Die experimentellen Daten, die durch die Tintenverfolgung, PTV sowie PIV gewonnen werden konnten, lassen sich gut durch die Simulationen reproduzieren. Gerade die anfänglich weniger aussagekräftigen Tintenfronten haben sich als nützlich erwiesen, um den nichtlinearen Ansatz bei den Simulationen $\mathrm{zu}$ validieren.

Bisher wurde nur das Limit der Schallwandler getestet sowie eine gute Beschreibung der ungestörten Strömung (experimentell und simuliert) erarbeitet. Eine erste Beschreibung der Strömung in der Nähe einer Grenzfläche wurde mittels PIV und vergleichender Simulation sowie durch Chronoamperometrie durchgeführt.

Viele mögliche Anwendungen sind noch nicht wirklich untersucht, aber im folgenden Ausblick werden einige erste Tests vorgestellt. 
5. Fazit 


\section{Ausblick}

Die durch die Schallwandler erzeugte akustische Strömung wird bisher noch für keine technische Anwendung genutzt. In diesem kurzen Ausblick werden einige Anwendungsmöglichkeiten skizziert und kurz getestet.

\subsection{Tropfenerzeugung}

Durch den räumlich und zeitlich begrenzten Jet, bzw. die abgelösten Wirbel lassen sich gezielt Tropfen aus einer Flüssigkeit schießen. Eine mögliche Anwendung wäre eine Art Tintenstrahldrucker, der ohne Aufheizen der Tinte auskommt, was z.B. für das gezielte Aufbringen von Zellkulturen nützlich sein könnte.

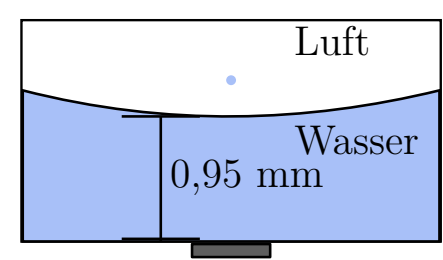

Schallwandler

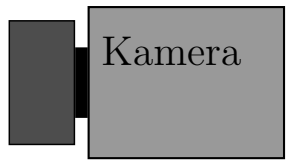

Abb. 6.1.: Skizze der Wasserschicht, aus der Tropfen nach oben herausgeschleudert wurden; der Schallwandler befindet sich unten in der Mitte.

Für einen ersten Test wurde der Schallwandler mit einer circa $1 \mathrm{~mm}$ dicken Wasserschicht bedeckt, wie in Abbildung 6.1 gezeigt, und für 50 us betrieben. Dadurch wird die Flüssigkeit für die Dauer des Pulses über dem Wandler beschleunigt und bildet, wie in Abschnitt 4.4 .4 gezeigt, einen Wirbel aus. Diese Strömung durchdringt die Wasseroberfläche und erzeugt einen Tropfen mit einer Geschwindigkeit von $\approx 1,4 \mathrm{~m} / \mathrm{s}$, wie in Abbildung 6.2 oben gezeigt. 


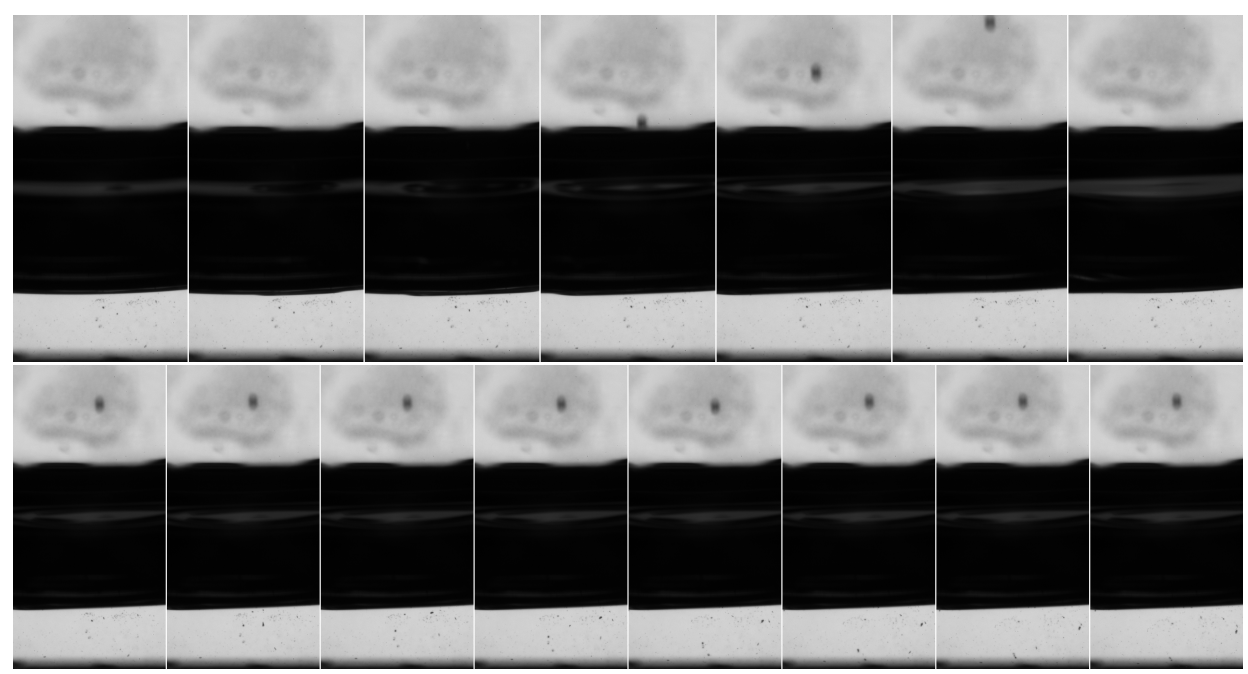

Abb. 6.2.: Wassertropfen Ausstoß durch einen 50 ps Puls. Der Bildausschnitt ist $2,5 \mathrm{~mm} \times 5 \mathrm{~mm}$ groß; die obere Reihe zeigt die Entstehung eines einzelnen Tropfens, Aufnahme mit 1750 Bildern pro Sekunde bzw. $\approx 0,57 \mathrm{~ms}$ zwischen zwei Bildern. Die untere Reihe zeigt unterschiedliche Tropfen zum gleichen Zeitpunkt nach dem Puls.

Die Erzeugung der Tropfen ist sehr reproduzierbar, wie in der unteren Zeile von Abbildung 6.2 zu sehen ist. Die gezeigten Tropfen haben einen Durchmesser von $140 \mu \mathrm{m}$, was einem Volumen von $0,0015 \mathrm{~mm}^{3}$ bzw. 1,5 nl entspricht. Je nach Pulslänge, angelegter Spannung am Schallwandler und Füllhöhe des Wassers werden unterschiedlich große und schnelle Tropfen erzeugt.

Dasselbe Prinzip wie mit der Luft-Wasser Grenzschicht funktioniert auch mit anderen Medien. In der folgenden Abbildung 6.3 wurden Wassertropfen in Silikonöl erzeugt. Durch den Dichteunterschied befindet sich das Öl über dem Wasser und am unteren Bildrand befindet sich der Schallwandler. Es lassen sich gezielt Wassertropfen in das Öl drücken und durch weitere Pulse kann man weiteres Wasser zu dem Tropfen hinzufügen. In einer MikrofluidikAnwendung könnten mit dieser Technik Tropfen erzeugt werden, sowie gezielt weitere Flüssigkeiten in die Tropfen eingebracht werden. Die elektrische Ansteuerung erlaubt eine gute Kontrolle über die Flüssigkeitsmengen. 


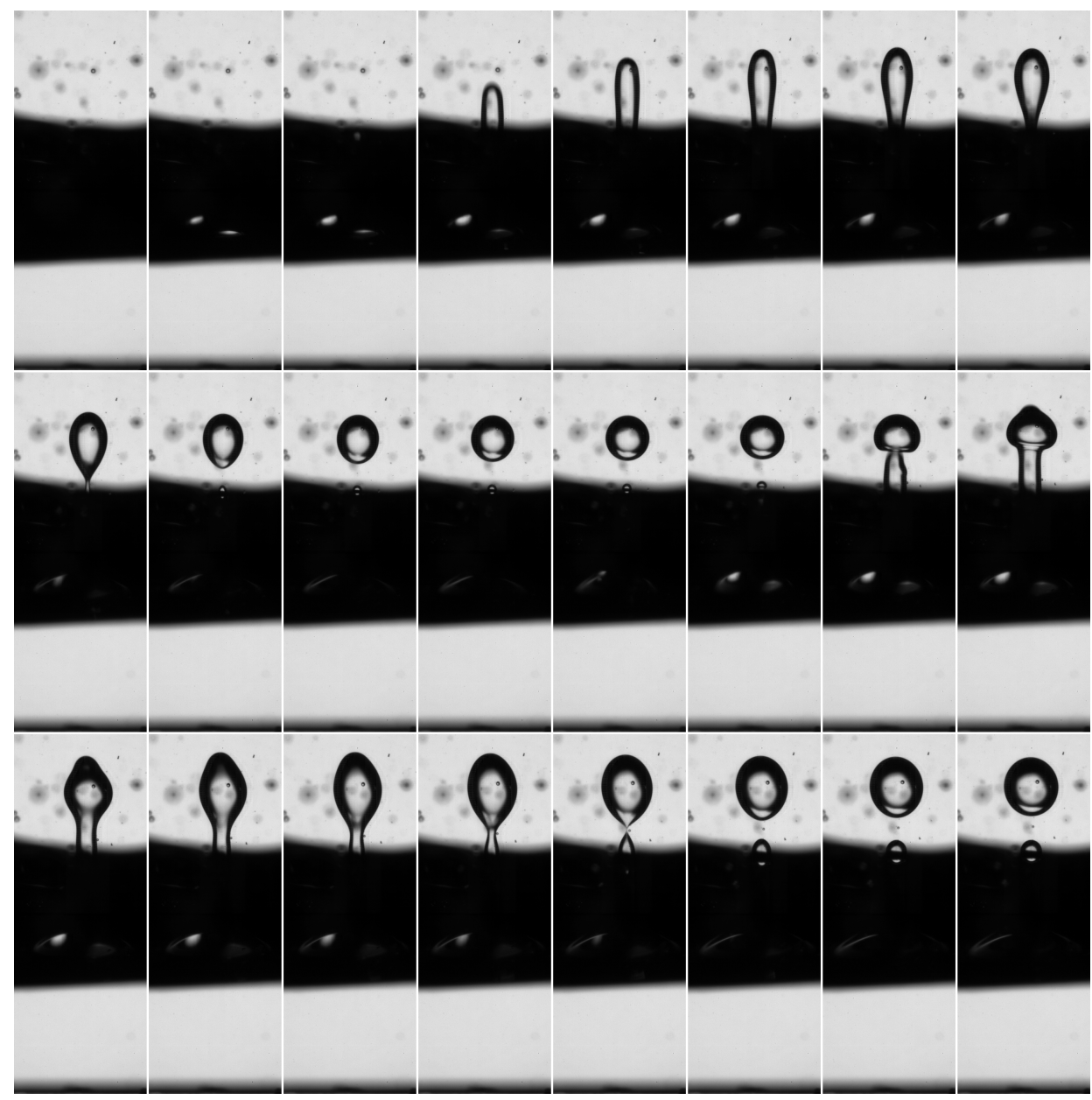

Abb. 6.3.: Wassertropfen in Silikonöl. Ein Bildausschnitt ist $1,84 \mathrm{~mm} \times 5 \mathrm{~mm}$ groß, Zeit zwischen zwei Bildern beträgt 0,6 ms.

Der in diesem Beispiel erzeugte Tropfen in Abbildung 6.3 hat nach dem ersten Puls ein Volumen von $0,11 \mu \mathrm{l}$ und nach dem zweiten Puls hat sich das Volumen auf circa $0,22 \mu \mathrm{l}$ verdoppelt.

\subsection{Mikropumpe / Flüssigkeitsjet}

Neben der Erzeugung von Tropfen kann die akustisch erzeugte Volumenkraft auch als eine Art Mikropumpe eingesetzt werden. Durch längere Pulse können Flüssigkeitsjets gebildet werden, wie in Abbildung 6.5 gezeigt. Der Jet in Abbildung 6.5 breitet sich anfangs mit über $1,5 \mathrm{~m} / \mathrm{s}$ aus und zerfällt durch Rayleigh-Taylor Instabilitäten in Tropfen.

Durch eine dünne rechteckige Glasröhre (mit abgerundeten Ecken) wie in der Skizze 6.4 gezeigt, kann die Grenzfläche zwischen Wasser und Luft gezielt in die Nähe des Schallwandlers gebracht werden. 


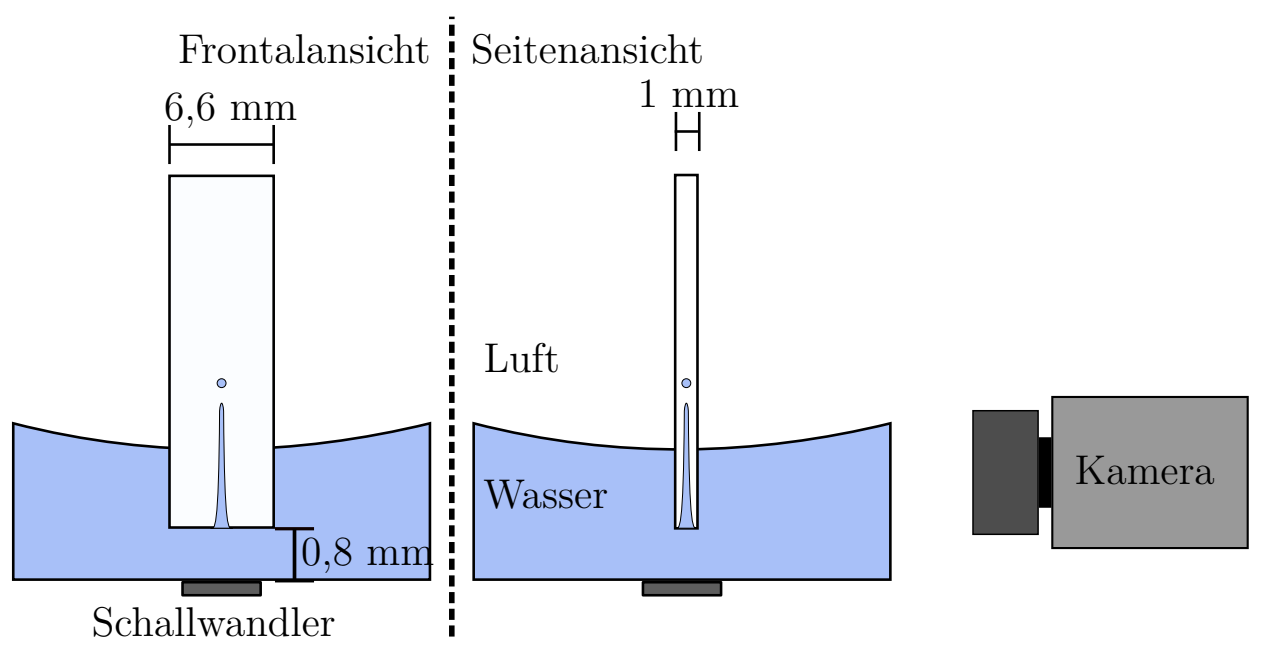

Abb. 6.4.: Skizze des Versuchsaufbaus in Frontal- und Seitenansicht.

Die Oberflächenspannung des Wassers ist ausreichend groß um das an beiden Seiten offene Röhrchen mit Luft gefüllt zu halten während es einige Millimeter ins Wasser getaucht wird. Dadurch wird die Stelle an der der Jet die Wasseroberfläche durchdringt von dem durch den Meniskus erzeugten dicken schwarzer Balken in den Aufnahmen, verschoben.

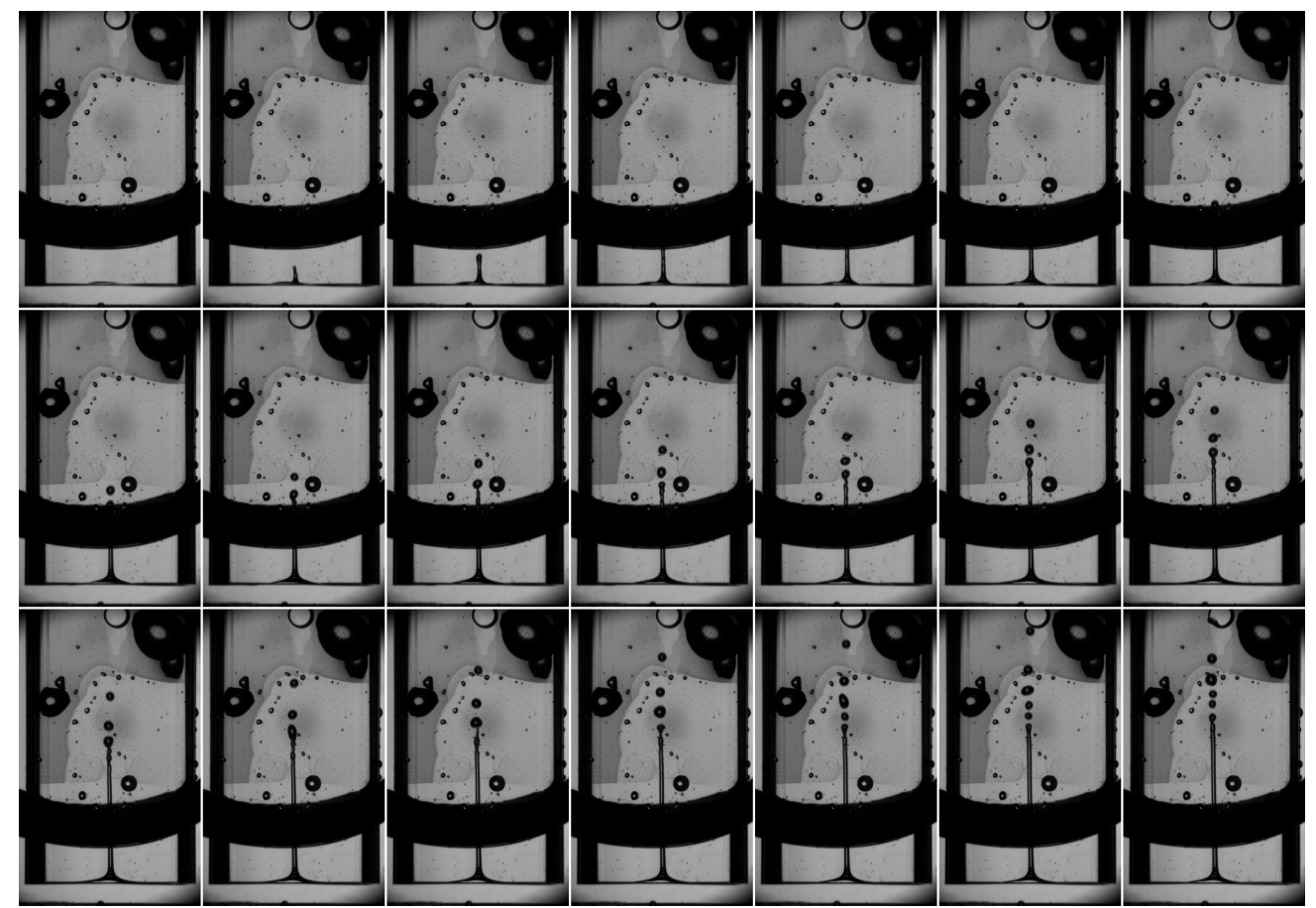

Abb. 6.5.: Flüssigkeitsjet während eines $100 \mathrm{~ms}$ Pulses. Ein Bildausschnitt ist $7,08 \mathrm{~mm} \times 11,5 \mathrm{~mm}$ groß, zeit zwischen zwei Bildern beträgt 0,2 ms, bzw. Aufnahme mit 5.000 Bilder pro Sekunde 


\subsection{Strömung in Spalten}

Auch die Strömung in einer Spalte wurde getestet. Dazu wurde eine dünne Spalte wie in Skizze 6.6 hergestellt und mit Tinte befüllt.

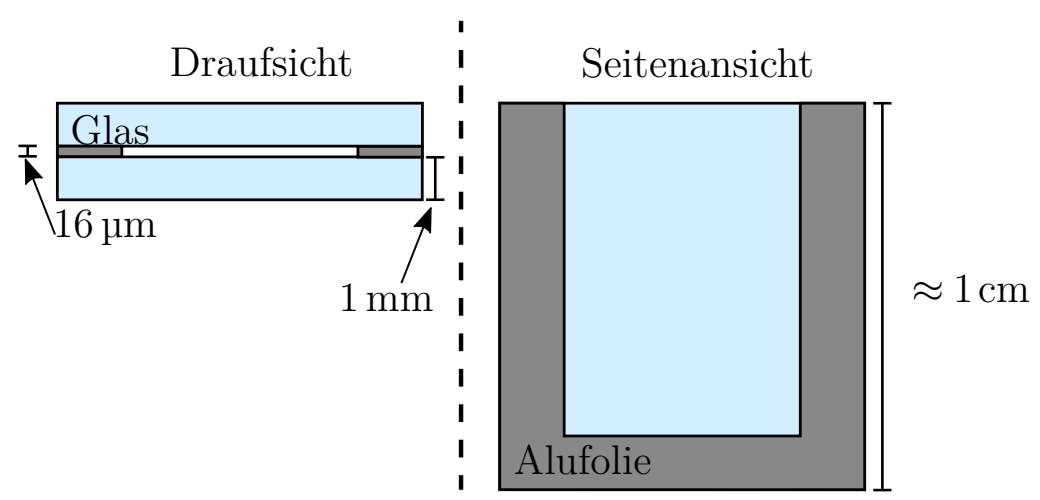

Abb. 6.6.: Skizze der Spalte

Die mit Tinte gefüllte Spalte wird für den Versuch in eine mit Wasser gefüllte Küvette getaucht und der Schallwandler darüber platziert. Die Abbildung 6.7 zeigt die Bilderserie eine Hochgeschwindigkeitsaufnahme. Der Schallwandler befindet sich oben mittig in der Abbildung. An den Schallwandler wird für $1 \mathrm{~ms}$ ein Sinus mit einer Frequenz von $785 \mathrm{MHz}$ angelegt mit einer Spannung $U_{p p} \approx 50 \mathrm{~V}$.

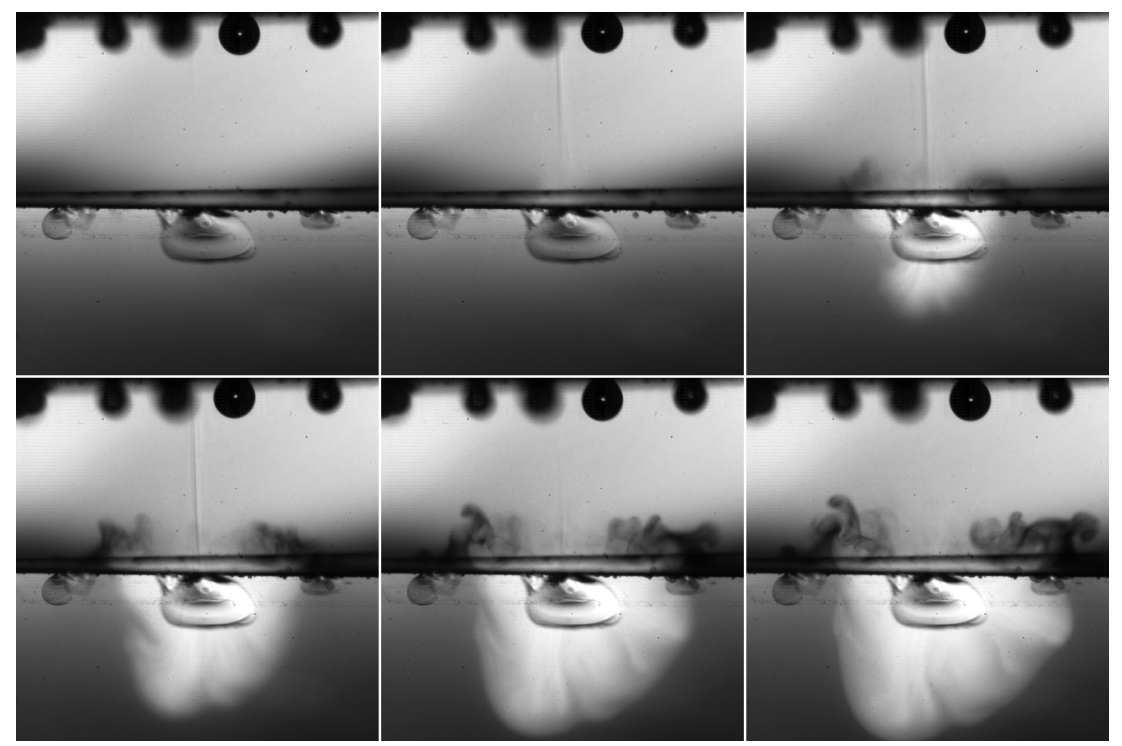

Abb. 6.7.: Bildserie einer Hochgeschwindigkeitsaufnahme, 5000 Bilder pro Sekunde. Die Breite und Höhe eines Bildausschnitts beträgt 1,96 mm.

Durch Schlieren ist die Flüssigkeitsströmung zu erkennen und mit Auftreffen auf die Spalte wird dort Tinte durch das Wasser verdrängt. 
Bei der Herstellung von Halbleiterchips geht die Entwicklung zu drei Dimensionalen Strukturen. Das Ätzen innerhalb der Strukturen sowie die Reinigung ist oft Problematisch und könnte möglicherweise durch akustische Strömung optimiert werden. Jedoch sind die dort vorhandenen Strukturen eine Größenordnung kleiner als in dem oberen Beispiel. 


\section{Literaturverzeichnis}

[1] David T. Blackstock. Fundamentals of physical acoustics. Wiley New York, 2000.

[2] edited by Mark F. Hamilton and David T. Blackstock. Nonlinear acoustics. Academic Press, San Diego, CA, 1998.

[3] L.D. Landau and E.M. Lifshitz. Fluid Mechanics: Landau and Lifshitz: Course of Theoretical Physics. Elsevier Science, 2013.

[4] James Friend and Leslie Y. Yeo. Microscale acoustofluidics: Microfluidics driven via acoustics and ultrasonics. Rev. Mod. Phys., 83:647-704, Jun 2011.

[5] M. S. Howe. Hydrodynamics and Sound. Cambridge University Press, 2006.

[6] Werner Lauterborn (et al.). Springer Handbook of Acoustics, chapter 8: Nonlinear Acoustics in Fluids, pages 265-314. Springer New York, 2014.

[7] R.T. Beyer and United States. Naval Ship Systems Command. Nonlinear Acoustics. The Command, 1974.

[8] WESLEY LE MARS NYBORG. 11 - acoustic streaming. In WARREN P. MASON, editor, Properties of Polymers and Nonlinear Acoustics, volume 2 of Physical Acoustics, pages 265 - 331. Academic Press, 1965.

[9] Frederick V. Hunt. Notes on the exact equations governing the propagation of sound in fluids. The Journal of the Acoustical Society of America, 27(6):1019-1039, 1955.

[10] P.M. Morse and K.U. Ingard. Theoretical Acoustics. International series in pure and applied physics. McGraw-Hill, 1971.

[11] Michel Bruneau. Basic Solutions to the Equations of Linear Propagation in Cylindrical and Spherical Coordinates, pages 227-275. ISTE, 2010.

[12] J.D.N. Cheeke. Fundamentals and Applications of Ultrasonic Waves, Second Edition. Taylor \& Francis, 2012. 
[13] B. Moudjed, V. Botton, D. Henry, H. Ben Hadid, and J.-P. Garandet. Scaling and dimensional analysis of acoustic streaming jets. Physics of Fluids, 26(9), 2014.

[14] Ph. M. Nasch, M. H. Manghnani, and R. A. Secco. A modified ultrasonic interferometer for sound velocity measurements in molten metals and alloys. Review of Scientific Instruments, 65(3):682-688, 1994.

[15] Kerstin Avila, David Moxey, Alberto de Lozar, Marc Avila, Dwight Barkley, and Björn Hof. The onset of turbulence in pipe flow. Science, 333(6039):192-196, 2011.

[16] K. Naugolnykh and L. Ostrovsky. Nonlinear Wave Processes in Acoustics. Cambridge Texts in Applied Mathematics. Cambridge University Press, 1998.

[17] J.M. Burgers. A mathematical model illustrating the theory of turbulence. Advances in Applied Mechanics, 1:171 - 199, 1948.

[18] Bengt O. Enflo and Claes M. Hedberg. Fourier decomposition of a plane nonlinear sound wave developing from a sinusoidal source. Acta Acustica united with Acustica, 87(2):163-169, 2001.

[19] E Fubini-Ghiron. Anomalies in acoustic wave propagation of large amplitude. Alta Frequenza, 4:530-581, 1935.

[20] David T Blackstock. Propagation of plane sound waves of finite amplitude in nondissipative fluids. The Journal of the Acoustical Society of America, 34(1):9-30, 1962.

[21] Winfield Keck and Robert T Beyer. Frequency spectrum of finite amplitude ultrasonic waves in liquids. Physics of Fluids (1958-1988), 3(3):346352,1960 .

[22] Logan E Hargrove. Fourier series for the finite amplitude sound waveform in a dissipationless medium. The Journal of the Acoustical Society of America, 32(4):511-512, 1960.

[23] Bill D Cook. New procedure for computing finite-amplitude distortion. The Journal of the Acoustical Society of America, 34(7):941-946, 1962.

[24] David T. Blackstock. Thermoviscous attenuation of plane, periodic, finite-amplitude sound waves. The Journal of the Acoustical Society of America, 36(3):534-542, 1964.

[25] R. D. Fay. Plane sound waves of finite amplitude. The Journal of the Acoustical Society of America, 3(2A):222-241, 1931. 
[26] David T. Blackstock. Connection between the fay and fubini solutions for plane sound waves of finite amplitude. The Journal of the Acoustical Society of America, 39(6):1019-1026, 1966.

[27] B.O. Enflo and C.M. Hedberg. Theory of Nonlinear Acoustics in Fluids. Fluid Mechanics and Its Applications. Springer Netherlands, 2002.

[28] Julian D. Cole. On a quasi-linear parabolic equation occuriring in aerodynamics. Quarterly of Applied Mathematics, 9(3):225-236, 1951.

[29] Eberhard Hopf. The partial differential equation ut + uux $=\hat{\mathrm{I}} 1 / 4 \mathrm{xx}$. Communications on Pure and Applied Mathematics, 3(3):201-230, 1950.

[30] J. S. Mendousse. Nonlinear dissipative distortion of progressive sound waves at moderate amplitudes. The Journal of the Acoustical Society of America, 25(1):51-54, 1953.

[31] L. K. Zarembo. Acoustic Streaming, pages 135-199. Springer US, Boston, MA, 1971.

[32] Lord Rayleigh. On the pressure developed in a liquid during the collapse of a spherical cavity. Phil. Mag., 34(6):94-98, 1917.

[33] Carl Eckart. Vortices and streams caused by sound waves. Phys. Rev., 73:68-76, Jan 1948.

[34] L. E. Kinsler, A. R. Frey, A. B. Coppens, and J. V. Sanders. Fundamentals of Acoustics, 4th Edition. Wiley New York, December 1999.

[35] J. B. Keller and M. Miksis. Bubble oscillations of large amplitude. J. Acoust. Soc. Am., 68(2):628-633, 1980.

[36] W. Lauterborn, T. Kurz, R. Mettin, and C. D. Ohl. Advances in Chemical Physics, volume 110, chapter Experimental and Theoretical Bubble Dynamics, pages 295-380. Wiley, 1999.

[37] U. Parlitz et al. Spatio-temporal dynamics of acoustic cavitation bubble clouds. Phil. Trans. R. Soc., 357:313-334, 1999.

[38] M. Minnaert. On musical air-bubbles and the sounds of running water. Phil. Mag., 16(7):235-248, 1933.

[39] F. R. Young. Cavitation. McGraw-Hill Book Company, London, 1989.

[40] F. G. Blake. The onset of cavitation in liquids. i. cavitation threshold sound pressures in water as a function of temperature and hydrostatic pressure. Technical Report 12, Harvard University, Acoustic Research Laboratory, 1949. 
[41] R. Courant H. Lewy, K. Friedrichs. über die partiellen differenzengleichungen der mathematischen physik. Mathematische Annalen, 100:32-74, 1928.

[42] H. Fellouah, C.G. Ball, and A. Pollard. Reynolds number effects within the development region of a turbulent round free jet. International Journal of Heat and Mass Transfer, 52(17):3943 - 3954, 2009. Special Issue Honoring Professor D. Brian Spalding.

[43] Michael C. Rogers and Stephen W. Morris. Natural versus forced convection in laminar starting plumes. Physics of Fluids, 21(8):083601, 2009.

[44] Elisha Moses, Giovanni Zocchi, and Albert Libchaberii. An experimental study of laminar plumes. Journal of Fluid Mechanics, 251:581â€"601, 1993.

[45] E J List. Turbulent jets and plumes. Annual Review of Fluid Mechanics, 14(1):189-212, 1982.

[46] Seok Jae Kwon and Il Won Seo. Reynolds number effects on the behavior of a non-buoyant round jet. Experiments in Fluids, 38(6):801-812, Jun 2005 .

[47] J.S. Seybold. Introduction to RF Propagation. Wiley, 2005.

[48] I.F. Sbalzarini and P. Koumoutsakos. Feature point tracking and trajectory analysis for video imaging in cell biology. Journal of Structural Biology, 151(2):182 - 195, 2005.

[49] W.S Rasband. ImageJ, U. S. National Institutes of Health, Bethesda, Maryland, USA, 1997-2012. http://imagej.nih.gov/ij/.

[50] W. Thielicke and E. J. Stamhuis. Pivlab- towards user-friendly, affordable and accurate digital particle image velocimetry in matlab. Journal of Open Research Software, e30(2), 2014.

[51] Fabian Reuter, Carlos Cairós, and Robert Mettin. Vortex dynamics of collapsing bubbles: Impact on the boundary layer measured by chronoamperometry. Ultrasonics Sonochemistry, 33:170 - 181, 2016.

[52] V. G. Levich. Physicochemical hydrodynamics, volume 2nd ed. PrenticeHall, 1962.

[53] Joseph Wang. Fundamental Concepts, chapter 1, pages 1-28. John Wiley \& Sons, Ltd, 2006. 
[54] C. Beriet and D. Pletcher. A microelectrode study of the mechanism and kinetics of the ferro/ferricyanide couple in aqueous media: The influence of the electrolyte and its concentration. Journal of Electroanalytical Chemistry, 361(1):93 - 101, 1993.

[55] Fabian Reuter and Robert Mettin. Electrochemical wall shear rate microscopy of collapsing bubbles. Phys. Rev. Fluids, 3:063601, Jun 2018.

[56] R Mettin C Cairós, T Nowak. Studying cavitation and acoustic streaming by electrochemistry. DAGA, 2012.

[57] Markus Kauer, Valentina Belova-Magri, Carlos Cairós, Hans-Jürgen Schreier, and Robert Mettin. Visualization and optimization of cavitation activity at a solid surface in high frequency ultrasound fields. Ultrasonics Sonochemistry, 34:474 - 483, 2017. 


\section{Literaturverzeichnis}




\section{A. Symbolliste}

Die folgende Liste enthält alle in der Arbeit verwendeten Symbole und ihre Bedeutung.

\begin{tabular}{cl} 
Symbol & Beschreibung \\
\hline$A$ & Parameter der Zustandsgleichung, siehe 2.1.9 \\
$B$ & Parameter der Zustandsgleichung, siehe 2.1 .10 \\
$D_{s}$ & Durchmesser des Schallwandlers \\
$F$ & Volumenkraft \\
$H$ & Heaviside Funktion \\
$I$ & Intensität \\
$I_{n}$ & modifizierte Besselfunktion $n$-ter Ordnung \\
$J_{n}$ & sphärische Besselfunktion $n$-ter Ordnung \\
$M a$ & Machzahl, siehe 2.5.3 \\
$N$ & Vielfache, bzw . N-fache eines Wertes \\
$N_{\alpha}$ & $\alpha / f^{2}$ Dämpfungskoeffizient \\
$Q$ & Parameter der Taitgleichung \\
$R$ & Richtcharakteristik \\
$R_{\text {krit }}$ & kritischer Radius \\
$R_{n}$ & Ruheradius einer Blase \\
$R e_{\mathrm{ak}}$ & akustische Reynoldszahl, siehe 2.5 .2 \\
$R e_{\text {hy }}$ & hydrodynamische Reynoldszahl, siehe 2.5 .1 \\
$T$ & Periodendauer Schallfeld \\
$U$ & dimensionslose Geschwindigkeit $u / u_{0}$ \\
$a$ & Beschleunigung der Auslenkung \\
$b$ & Viskosität (modifiziert durch Wärmeleitungseffekte) \\
$c$ & Schallgeschwindigkeit \\
$c_{0}$ & Schallgeschwindigkeit in Ruhe \\
$c_{p}$ & spezifische Wärmekapazität bei konstantem Druck \\
$c_{v}$ & spezifische Wärmekapazität bei konstantem Volumen \\
$f$ & Frequenz \\
$p$ & Druck \\
$p_{0}$ & Druck in Ruhe \\
$p_{B d}$ & dynamischer Blake Druck, siehe 2.8.5 \\
$p_{B s}$ & statischer Blake Druck, siehe 2.8 .4 \\
$r$ & Abstand von der Achse der Rotationssymmetrie \\
$s$ & Entropie \\
$t$ & Zeit \\
&
\end{tabular}




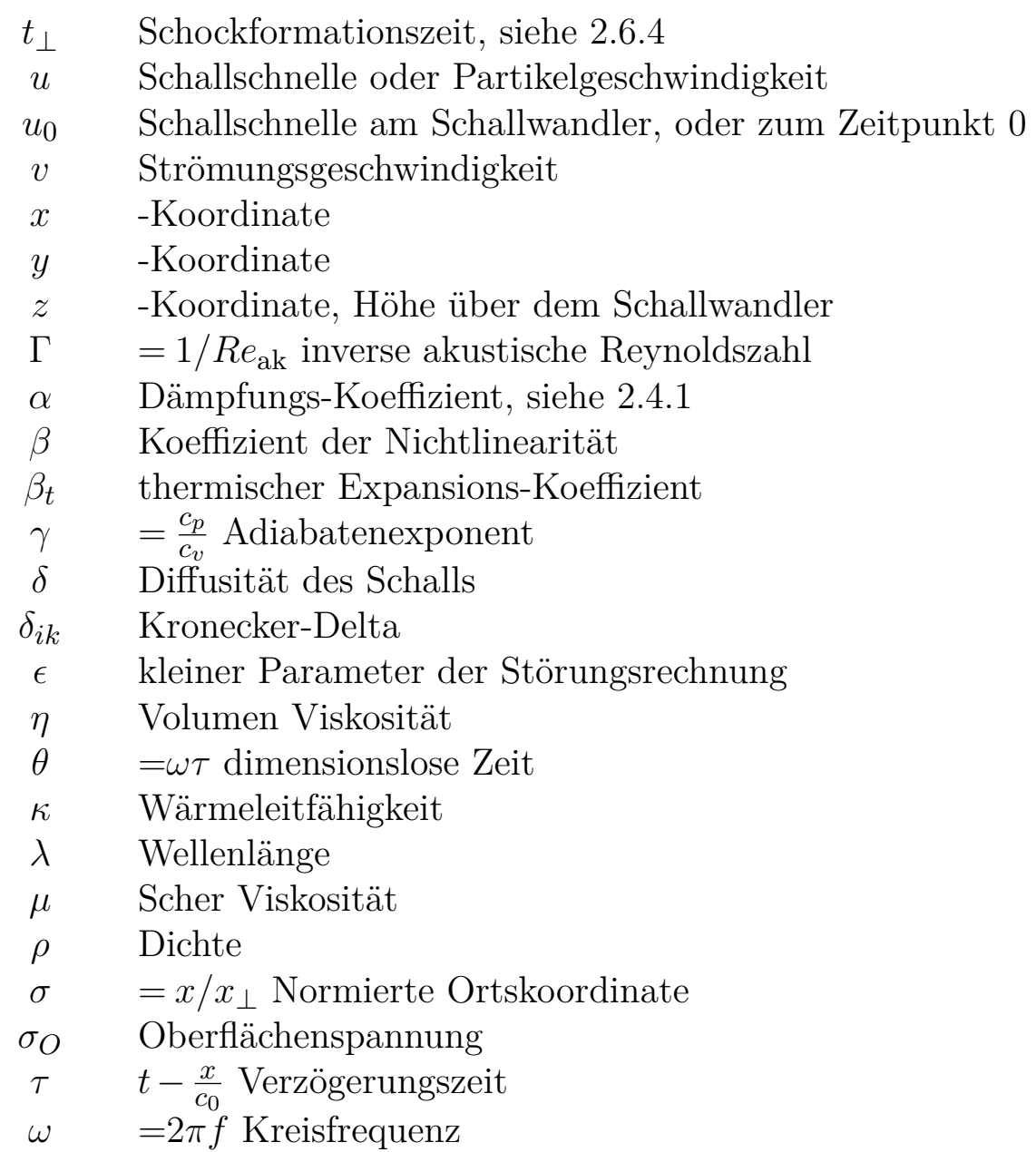




\section{B. Danksagung}

Viele Personen und Institutionen haben mir die Experimente, die dieser Dissertation zugrunde liegen, sowie die sich anschließende Verschriftlichung ermöglicht und mich unterstützt; diesen möchte ich an dieser Stelle danken:

Meinen Betreuern Prof. Dr. Christoph Schmidt, Prof. Dr. Jörg Enderlein und Prof. Dr. Ulrich Parlitz für die Betreuung. Ferner möchte ich einen besonderen Dank aussprechen an Dr. Robert Mettin für die stetige Betreuung, für die vielen Anregungen experimenteller wie nummerischer Art, sowie für einen abschließenden kritischen Blick auf diese Dissertationsschrift.

Die ausgezeichnete Infrastruktur des DPI ermöglichte erst den Experimentaufbau. Die feinmechanische Werkstatt unter Leitung von Dieter Hille, sowie die Elektronikwerkstatt unter der Leitung von Markus Schönekeß haben mit allen ihren Mitarbeiter stets problemorientiert mitgewirkt und so eine rasche, Durchführung der Experimente gewährleistet, sowie Thomas Geiling, der ein störungsfreies Funktionieren der IT sicherstellt.

Dem wechselnden Sekretariat Elke Zech, Sabine Huhnold, Ursula Hahn-Wörgötter und Regina Wunderlich, die einen immer freundlich bei der notwendigen Bürokratie unterstützen.

Der Christian Doppler Forschungsgesellschaft für das CD-Labor für Kavitation und Mikroerosion in dem diese Dissertation begonnen wurde. Und natürlich auch dem Industriepartner LAM-Research mit Dr. Philipp Frommhold und den ehemaligen Mitarbeitern Alex Lippert, sowie Dr. Harald Okorn-Schmidt für den fachlichen Austausch und die finanzielle Unterstützung des Projektes.

Dem Fraunhofer-Institut für Biomedizinische Technik IBMT in St. Ingbert mit Anette Jakob für die neuartigen Schallwandler sowie der wissenschaftlichen Expertise bei deren Betrieb.

Natürlich auch der gesamten „Bubbles“-Arbeitsgruppe mit dem auch im Ruhestand noch präsenten Prof. Dr. Werner Lauterborn und allen ehemaligen sowie aktuellen Mitgliedern wie Carlos, Dwayne, Ekim, Fabian, J, Julia, Karsten, Markus, Max Shir, Miquel, Philipp und Till für all die hilfreichen und interessanten Gespräche, Diskussionen und Experimente. 
B. Danksagung

Schließlich auch meinen Eltern für die Unterstützung über das gesamte Studium hinweg bis zur abschließenden Fehlerkorrektur. 